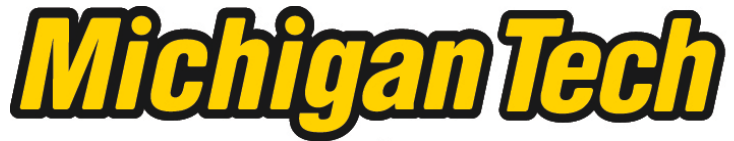 \\ Michigan Technological University Create the Future Digital Commons @ Michigan Tech
}

Dissertations, Master's Theses and Master's Reports - Open

Dissertations, Master's Theses and Master's

Reports

2010

Edge coloring BIBDS and constructing MOELRs

John S. Asplund

Michigan Technological University

Follow this and additional works at: https://digitalcommons.mtu.edu/etds

Part of the Mathematics Commons

Copyright 2010 John S. Asplund

\section{Recommended Citation}

Asplund, John S., "Edge coloring BIBDS and constructing MOELRs ", Master's Thesis, Michigan Technological University, 2010.

https://doi.org/10.37099/mtu.dc.etds/202

Follow this and additional works at: https://digitalcommons.mtu.edu/etds

Part of the Mathematics Commons 


\title{
Edge Coloring BIBDs and Constructing MOELRs
}

\author{
By \\ JOHN S. ASPLUND
}

\begin{abstract}
A THEsis
Submitted in partial fulfillment of the requirements for the degree of MASTER OF SCIENCE IN MATHEMATICAL SCIENCES

MICHIGAN TECHNOLOGICAL UNIVERSITY 2010
\end{abstract}

(C) 2010 John S. Asplund 

This thesis, "Edge Coloring BIBDs and Constructing MOELRs", is hereby approved in partial fulfillment of the requirements for the degree of MASTER OF SCIENCE IN MATHEMATICAL SCIENCES.

DEPARTMENT:

Mathematical Sciences

Signatures:

Thesis Advisor

Dr. Melissa Keranen

Department Chair

Dr. Mark Gockenbach

Date 



\section{Acknowledgements}

I would like to dedicate this first to Nancy Lachapelle for her everlasting support and love. Thank you darling. Thank you Mom and Dad for all the visits and trips to Walmart and the support you have given me. They did not go unnoticed. Dave Kamin, I wish you could be here to right now. It has been too long since our last talk. I am sure I speak for your family when I say we miss you and await your return. Make sure you bring back souvenirs. I would also like to thank Diane Gutekunst for supporting me throughout my graduate career. You have been able to support me from afar with your bountiful gifts of assorted candies, goodies, and words of encouragement. Thank you for all that you have given me.

Next I would to thank Melissa Keranen for her support and assistance throughout this book. Without you, this would not have been possible. I know I put a lot of strain on you with the constant questions especially since you are in the process of raising two beautiful children, Lily and Maija.

I would like to thank Don Kreher for his assistance in the beginning of Chapter 6, I would also like to thank Richard Fears with his assistance in creating bibdchecker.

Finally, I would like to thank everyone else that put up with my constant barrage of questions including Dave Clark, Richard Fears, Margaret Perander, Jeanne Meyers, and Tori Conners. Without all of you this may not have been possible. 


\section{Abstract}

Chapter 1 is used to introduce the basic tools and mechanics used within this thesis. Some historical uses and background are touched upon as well. The majority of the definitions are contained within this chapter as well.

In Chapter2 we consider the question whether one can decompose $\lambda$ copies of monochromatic $K_{v}$ into copies of $K_{k}$ such that each copy of the $K_{k}$ contains at most one edge from each $K_{v}$. This is called a proper edge coloring (Hurd, Sarvate, [29]). The majority of the content in this section is a wide variety of examples to explain the constructions used in Chapters 3 and 4 .

In Chapters 3 and 4 we investigate how to properly color $\operatorname{BIBD}(v, k, \lambda)$ for $k=4$, and 5. Not only will there be direct constructions of relatively small BIBDs, we also prove some generalized constructions used within.

In Chapter 5 we talk about an alternate solution to Chapters 3 and 4 , A purely graph theoretical solution using matchings, augmenting paths, and theorems about the edgechromatic number is used to develop a theorem that than covers all possible cases. We also discuss how this method performed compared to the methods in Chapters 3 and 4 .

In Chapter 6, we switch topics to Latin rectangles that have the same number of symbols and an equivalent sized matrix to Latin squares. Suppose $a b=n^{2}$. We define an equitable Latin rectangle as an $a \times b$ matrix on a set of $n$ symbols where each symbol appears either $\left\lceil\frac{b}{n}\right\rceil$ or $\left\lfloor\frac{b}{n}\right\rfloor$ times in each row of the matrix and either $\left\lceil\frac{a}{n}\right\rceil$ or $\left\lfloor\frac{a}{n}\right\rfloor$ times in each column of the matrix. Two equitable Latin rectangles are orthogonal in the usual way. Denote a set of $k a \times b$ mutually orthogonal equitable Latin rectangles as a $k-\operatorname{MOELR}(a, b ; n)$. We show that there exists a $k-\operatorname{MOELR}(a, b ; n)$ for all $a, b, n$ where $k$ is at least 3 with some exceptions. 


\section{Contents}

\begin{tabular}{|ll}
\hline Acknowledgements & i
\end{tabular}

Abstract ii

List of Figures $\quad$ v

1 Introduction 1

1.1 Graphs . . . . . . . . . . . . . . . . . . . 1

1.1 .1 Vertex-Colored Graphs . . . . . . . . . . . . . . . . 2

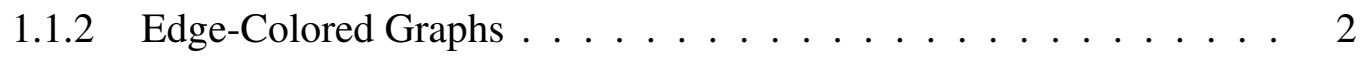

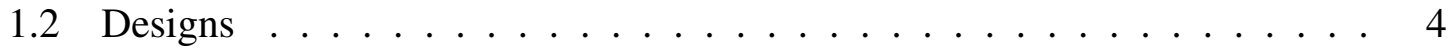

2 Proper Edge Colorings of $\operatorname{BIBD}(v, k, \lambda) \quad 11$

2.1 The Focus . . . . . . . . . . . . . . . . . . 11

2.2 Examples of Direct Constructions . . . . . . . . . . . 15

3 Proper Edge Colorings With Block Size 4 27

3.1 Direct Constructions With Block Size 4 . . . . . . . . . . . . . 27

3.2 Main Results $\ldots \ldots \ldots \ldots$. . . . . . . . . . . . . . . 35

$3.2 .1 \lambda \equiv 0(\bmod 3) \ldots \ldots \ldots \ldots$

$3.2 .2 \lambda \equiv 2,4(\bmod 6) \ldots \ldots \ldots \ldots \ldots$

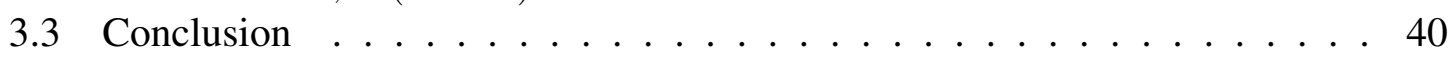

4 Proper Edge Coloring With Block Size 5 43

4.1 Direct Constructions With Block Size 5 . . . . . . . . . . . . . . . . . 43

4.2 Main Results . . . . . . . . . . . . . . . . . . . . 68

$4.2 .1 \quad \lambda \equiv 0(\bmod 2) \ldots \ldots \ldots \ldots \ldots$

$4.2 .2 \lambda \equiv 0(\bmod 4) \ldots \ldots \ldots \ldots \ldots$

4.3 Conclusion $\ldots \ldots \ldots \ldots \ldots \ldots$

5 Alternate Method to Properly Color $\quad 79$

5.1 Background in Graph Theory . . . . . . . . . . . . . . . . . . . . . 79

5.2 Restating our Problem . . . . . . . . . . . . . . . . . . . . 81

5.3 BIBD vs. PD . . . . . . . . . . . . . . . . 82 
6 Mutually Orthogonal Equitable Latin Rectangles 85

6.1 Mutually Orthogonal Latin Squares . . . . . . . . . . . . . . . . . . . 85

6.2 Background and Terms . . . . . . . . . . . . . . . . . . 88

6.3 Small Cases . . . . . . . . . . . . . . . . . . . . . 90

6.4 Computer Construction of MOELR . . . . . . . . . . . . . . . . . . . . . . . . . . . . . . 95

6.5 Supporting Lemmas . . . . . . . . . . . . . . . . . . . . . . . . . . . . . . . . . . . . . . . . . . . . . . . . . . . . . .

6.6 Main Theorem . . . . . . . . . . . . . . . . . . . . . . . . . . 114

\begin{tabular}{|ll}
\hline A Table of MOELR $(a, b ; n)$ where $n \leq 216$ & $\mathbf{1 2 3}$
\end{tabular}

\begin{tabular}{lr}
\hline Bibliography & 140
\end{tabular}

\begin{tabular}{ll}
\hline Index & 144
\end{tabular} 


\section{List of Figures}

1.1 A properly colored graph. . . . . . . . . . . . . . . . . . 3

1.2 A date-match problem . . . . . . . . . . . . . . . 3

1.3 Using augmenting paths on alternating path $P$ of matching $M \ldots \ldots$

1.4 The Fano plane. . . . . . . . . . . . . . . . . . . . . . . . . . 5

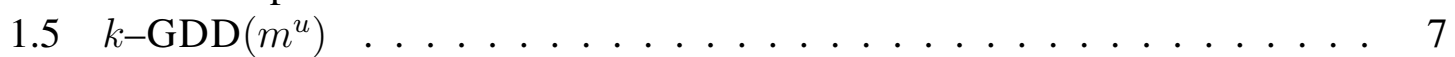

$1.6 \quad$ One block of a $\left.4-\operatorname{GDD}\left(4^{4}\right)\right] \ldots \ldots \ldots \ldots \ldots$

$2.11^{\text {st }}$ of three colored copies of a block from a $\operatorname{BIBD}(7,3,3) \ldots \ldots \ldots$

$2.22^{\text {nd }}$ of three colored copies of a block from a $\operatorname{BIBD}(7,3,3) \ldots \ldots \ldots \ldots$

$2.33^{\text {rd }}$ of three colored copies of a block from a $\operatorname{BIBD}(7,3,3) \ldots \ldots \ldots$

2.4 Incidence matrix of a $\operatorname{BIBD}(7,3,1) \ldots \ldots \ldots \ldots \ldots$

2.5 Edge-incidence matrix of a $\operatorname{BIBD}(7,3,1) \ldots \ldots \ldots \ldots$

2.6 A $7 \times 7$ circulant matrix. $\ldots \ldots \ldots \ldots \ldots \ldots \ldots \ldots \ldots$

$2.7 \quad$ Edge-colored incidence matrix of a $\operatorname{BIBD}(7,3,1) \ldots \ldots \ldots \ldots \ldots$

$2.8 \quad$ Edge-incidence matrix for a $\operatorname{BIBD}(5,4,3) \ldots \ldots \ldots \ldots \ldots$

$2.9 \quad$ Edge-Coloring Incidence Matrix for a $\operatorname{BIBD}(5,4,3) \quad \ldots \ldots \ldots \ldots$

2.10 Edge-Coloring Incidence Matrix of a properly colored $\operatorname{BIBD}(5,4,9) . \quad \ldots 20$

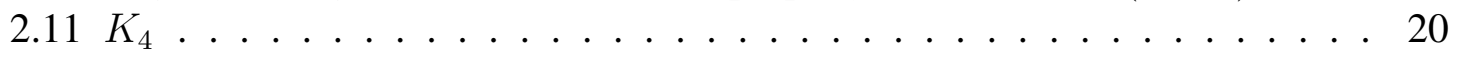

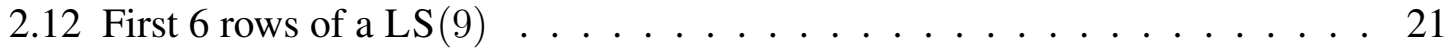

2.13 Coloring within groups of a GDD $\ldots \ldots \ldots \ldots \ldots \ldots$

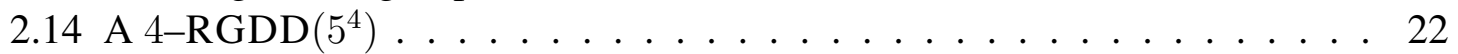

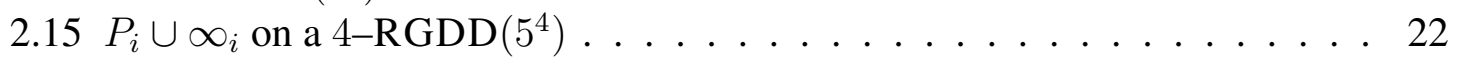

2.16 Edge-coloring incidence matrix for $(5,2)-$ GDD $\ldots \ldots \ldots \ldots \ldots$

2.17 Edge-coloring incidence matrix for $(5,2)-$ GDD with cyclic shifting $\ldots . .24$

$2.18 \mathrm{LS}(10)$ of 10 copies of block $B^{\prime} \ldots \ldots \ldots \ldots \ldots \ldots$

$3.1 \quad$ Edge-Coloring Incidence Matrix of a $\operatorname{BIBD}(9,4,3) . \ldots \ldots \ldots \ldots$

3.2 Edge-Coloring Incidence Matrix of a $\operatorname{BIBD}(8,4,3) . \ldots \ldots \ldots \ldots$

3.3 Edge-Coloring Incidence Matrix for a $\operatorname{BIBD}(8,4,3) \ldots \ldots \ldots$

3.4 Edge-Coloring Incidence Matrix for a $\operatorname{BIBD}(8,4,6) \quad \ldots \ldots \ldots \ldots$

3.5 Edge-Coloring Incidence Matrix for a $\operatorname{BIBD}(8,4,9) \quad \ldots \ldots \ldots \ldots$

$3.6 \quad$ Edge-Coloring Incidence Matrix of a $\operatorname{BIBD}(7,4,2) . \ldots \ldots \ldots \ldots \ldots$

3.7 Edge-Coloring Incidence Matrix of a $\operatorname{BIBD}(19,4,2) . \ldots \ldots \ldots \ldots$

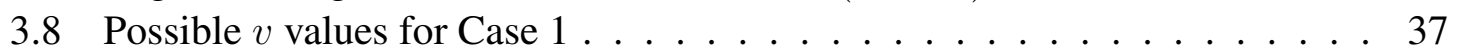


3.9 Possible $v$ values for Case $2 \| \ldots \ldots \ldots \ldots \ldots$

3.10 Possible $v$ values for Theorem 3.12 . . . . . . . . . . . . . . . 40

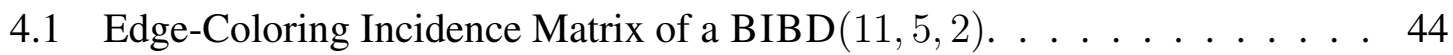

$4.2 \quad$ Edge-Coloring Incidence Matrix of a $\operatorname{BIBD}(31,5,2) . \quad \ldots \ldots \ldots$

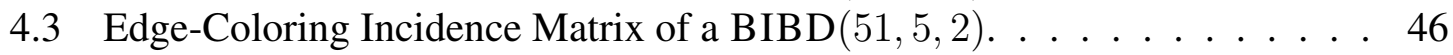

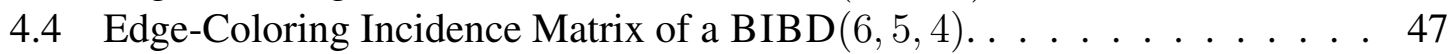

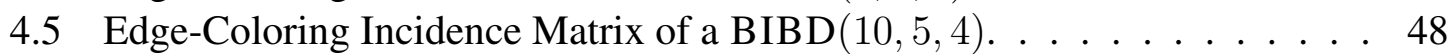

$4.6 \quad$ Edge-Coloring Incidence Matrix of a $\operatorname{BIBD}(20,5,4) . \quad \ldots \ldots$

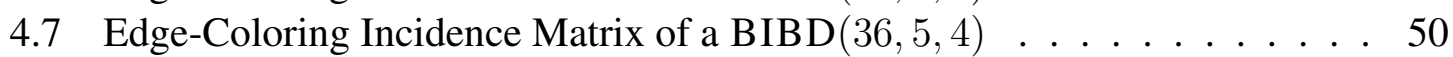

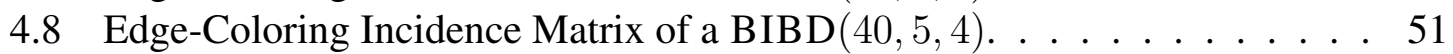

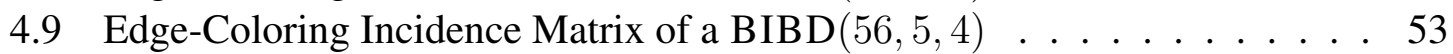

4.10 Edge-Coloring Incidence Matrix of a $\operatorname{BIBD}(56,5,4) \ldots \ldots$

4.11 Edge-coloring Incidence Matrix of a $\operatorname{BIBD}(15,5,6) \quad \ldots \ldots$

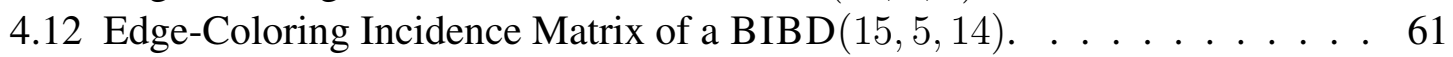

$4.1335 \times 28$ colored matrix $\ldots \ldots \ldots \ldots$. . . . . . . . . . 62

$4.1435 \times 28$ colored matrix $\ldots \ldots \ldots$. . . . . . . . . . . 63

4.15 Edge-Coloring Incidence Matrix of a $\operatorname{BIBD}(35,5,2) . \quad \ldots . . . .64$

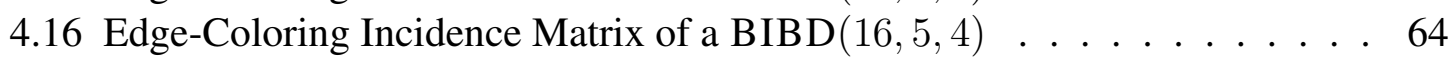

4.17 First of two edge-coloring incidence matrices of a $\operatorname{BIBD}(50,5,4) \ldots 66$

4.18 Second of two edge-coloring incidence matrices of a $\operatorname{BIBD}(50,5,4)$. . . 67

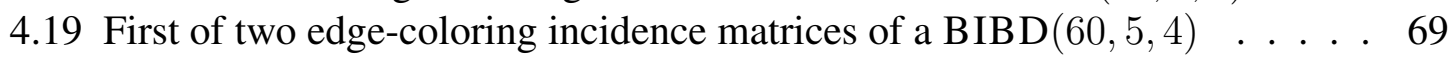

4.20 Second of two edge-coloring incidence matrices of a $\operatorname{BIBD}(60,5,4)$. . . 70

5.1 Proving the Marriage Theorem with alternating paths. . . . . . . . . . . 80

$6.1 \quad$ Latin square of side $8 \ldots \ldots \ldots \ldots$. . . . . . . . . . . . 85

$6.2 \quad \operatorname{A~PBD}(10,\{3,4\}) \ldots \ldots \ldots \ldots \ldots$

$6.3 \quad \mathrm{An} \mathrm{OA}(5,4) \ldots \ldots \ldots \ldots \ldots \ldots \ldots$

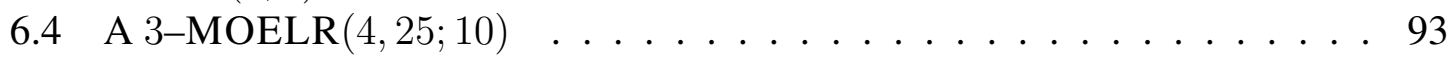

6.5 A $3-\operatorname{MOELR}(4,49 ; 14) \quad \ldots \ldots \ldots \ldots \ldots$

6.6 A $2-\operatorname{MOELR}(6,24 ; 12) \ldots \ldots \ldots \ldots$

$6.8 \mathcal{R}_{1}$ of matrix $X . \ldots \ldots \ldots \ldots \ldots$

6.7 The array $\mathcal{R}_{1}$ from $X . \ldots \ldots \ldots$. . . . . . . . . . . . . . . 99

6.9 Example of $\mathcal{R}_{1} \ldots \ldots \ldots \ldots \ldots$

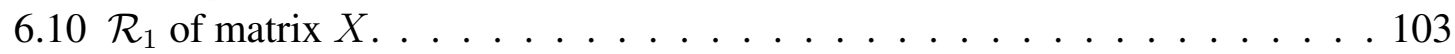

6.11 Form of $\mathcal{R}_{i}^{\prime \prime}$ to check equitability. . . . . . . . . . . . . . . . . . . . . 104

6.12 Form of $\mathcal{R}_{i}^{\prime \prime}$ to check equitability. . . . . . . . . . . . . . . . . 106

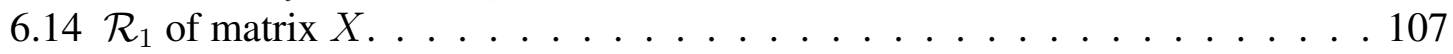

6.13 Example of $\mathcal{R}_{1} \ldots \ldots \ldots \ldots$. . . . . . . . . . . . . . . . . . 108

6.15 Converted $9 \times 9$ Latin square . . . . . . . . . . . . . . . . 110

6.16 Representation of the first mutually orthogonal Latin square of the $n \times n$

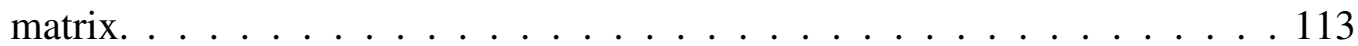

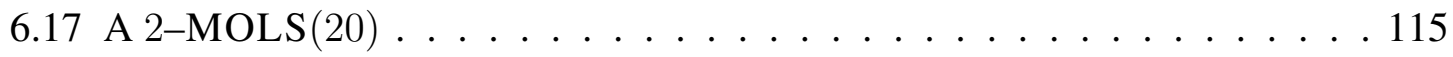


6.18 Permuted matrices of a 2-MOLS $(20) . \ldots \ldots$. . . . . . . . . . . . . . . . . . . . . . . . . . . . . .

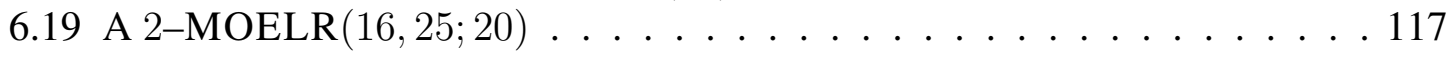




\section{Chapter 1}

\section{Introduction}

This thesis is the result of the author's interest in furthering recently developed fields in combinatorial designs. The goal of this thesis is to construct proper colorings of complete graphs using balanced incomplete block designs and expand the known results of mutually orthogonal equitable Latin rectangles. With that said, now we begin with an introduction to graph theory.

\subsection{Graphs}

To begin, a graph, $G$, is an ordered pair $(V, E)$ comprised of a set of vertices $V$ and a collection of unordered pairs of vertices called edges $E$. The order of the graph is $|V|$, the number of vertices. To be more precise, we will only deal with graphs that are simple and undirected. A simple graph is a graph with no loops or multiple edges between two distinct vertices. If an edge connects a vertex to itself we say the edge is a loop. A graph may have several edges between the same two vertices. These edges are called multiple edges. Figure 1.1 is an example of a simple graph. A simple graph is a complete graph if every pair of vertices is joined by an edge. The complete graph with $v$ vertices is denoted $K_{v}$.

Graph colorings have a wide variety of real world applications including radio wave assigning. Suppose we have six different radio stations at different distances from each other. If two radio stations are within 100 miles of each other then they cannot use the same frequency. To apply graph colorings to this problem, draw six vertices to represent the six radio stations. Place an edge between two vertices if they are within 100 miles of each other. The frequencies will be represented by colors. So if radio station $a$ and $b$ are within 100 miles, we need to color the vertices different colors because they cannot use the same frequency. In other words, if we give a coloring that uses the least number of colors possible on the vertices of the graph we created, we find the least number of radio signals we can possibly use without landing on some other stations signal. This is useful considering how many radio signals are continually flooding the air waves around populated areas such as Chicago and New York City.

There are several ways to color graphs including two of the most popular methods: vertex-coloring and edge-coloring. We are most interested in coloring the edges of a graph. 
Let $v$ be the number of vertices. The maximum number of edges possible in a simple graph is $\left(\begin{array}{l}v \\ 2\end{array}\right)=\frac{v(v-1)}{2}$. Much has been done in the field of graph coloring which is why we will touch on both of the two previously mentioned sub-divisions of graph coloring. For a more in-depth look into the properties, definitions, and theorems of graph theory, see [20].

\subsubsection{Vertex-Colored Graphs}

Two vertices are adjacent if they share a common edge. Two edges can be adjacent as well as long as they share a common vertex. Two edges are adjacent if they share a common vertex. A labeling of the vertices of a graph $G$ by the colors $\{1,2, \ldots, k\}$ in such a way that adjacent vertices receive different colors is called a $k$-coloring of $G$. The chromatic number, $\chi(G)$, of a graph $G$ is the smallest integer $k$ such that $G$ has a $k$-coloring.

There are many applications of vertex coloring. When scheduling a set of interfering jobs, one can use a conflict graph. A conflict graph is a simple graph where an edge between two vertices represents when two corresponding jobs are unable to be executed at the same time. This can happen if two jobs share a resource like machinery or personnel or interfere in some other way. Let the colors represent time slots and every job require one time slot. This creates a one to one correspondence with the vertices and colors because a vertex or a job can only be completed once. If the colors represent one hour intervals then the chromatic number is the minimum number of hours required to finish all $v$ jobs. For more examples of vertex-coloring see [35].

\subsubsection{Edge-Colored Graphs}

Intuitively, a proper edge-coloring is an assignment of colors to the edges of a graph such that no adjacent edges share a color. Formally, we may define a proper edge-coloring as follows.

Definition 1.1. Let $G=(V, E)$ be a graph and $C=\{1,2, \ldots, k\}$ be a set of colors. A proper coloring 1 or proper edge-coloring is a labeling of the edges with colors from $C$ in such a way where no two adjacent edges share a color in common.

If we properly color a complete graph $K_{k}$, each color is used at most once when coloring the edges of $K_{k}$ making each $K_{k}$ panchromatic. A graph is panchromatic if the graph has a unique color on each edge. Figure 1.1 depicts a properly colored graph of five vertices and six edges with minimum number of colors so that no two adjacent edges are given the same color.

A matching of a graph $G$ is a set of edges where no two edges share a vertex. Another way to say this is the graph has a 1-factor. Suppose we select the two blue edges of Figure 1.1. Since these two edges are non-adjacent, we have a matching. The same is true if we select the red or green sets of edges as well.

\footnotetext{
${ }^{1}$ For simplicity we will use this word rather than proper edge-coloring.
} 


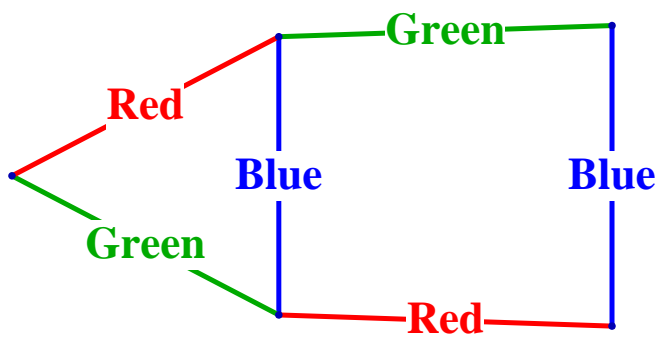

Figure 1.1: A properly colored graph.

Matching problems have many applications. We consider the date-match problem represented in Figure [1.2, an example from [39]. This figure is called a bipartite graph because

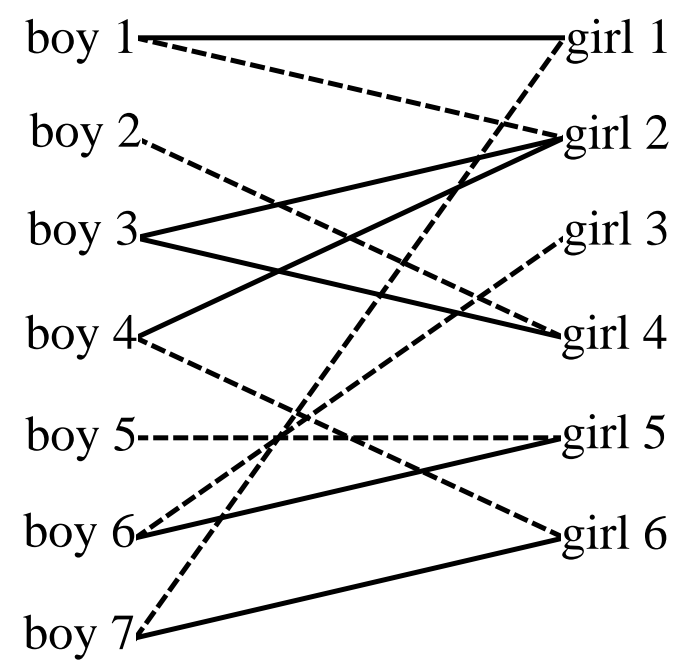

Figure 1.2: A date-match problem

it separates the vertices into two partition classes or sets where two vertices within the same partition class are non-adjacent. Suppose there are several boys and girls, and each girl favors some of the boys. What condition is necessary to make a date-matching in which every girl is assigned to one of her favorite boys? Consider a graph in which boys and girls correspond to vertices and a vertex corresponding to a girl is joined to vertices corresponding to boys whom the girl favors. Then there is a date-matching if and only if the graph has a matching containing all vertices corresponding to girls. The dashed edges give a possible matching given the requirements in the question.

Make note that if $S$ is a set, then $S \backslash\{u\}$ or $S-\{u\}$ means take $S$ and remove all elements of $\{u\}$ from $S$.

How do we generate a matching of $G$ with as many edges as possible? One such way is through the use of alternating paths and augmenting paths. First consider an arbitrary matching called $M$ which consists of edges from a graph $G$. A path in $G$ which starts at an 
unmatched vertex in $X$ and then contains an edge from $E \backslash M$, an edge from $M$, an edge from $E \backslash M$, an edge from $M$, and so on, is an alternating path with respect to $M$. An alternating path $P$ that ends in an unmatched vertex of the set $Y$ is called an augmenting path as seen in Figure 1.3. The bold edges on the left graph are the current matching. By removing the edges from the matching $M$ that are from the augmenting path $P$ in Figure 1.3 and add the edges that were not in $M$ but were in $P$, then the matching $M^{\prime}$ has a larger matching than $M$ as seen in the right graph in Figure 1.3 .

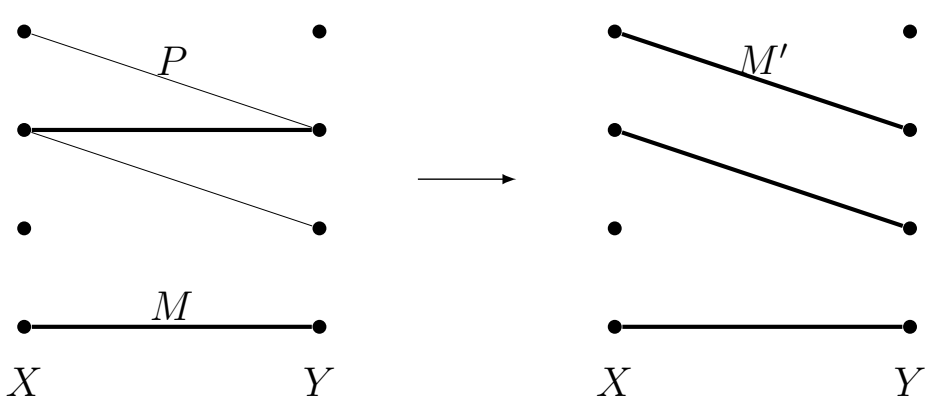

Figure 1.3: Using augmenting paths on alternating path $P$ of matching $M$

The first paper on edge-coloring problems was published by Tait back in 1889. In this paper he proved that the four-color conjecture is true if and only if one can edge-color every planar 3-connected cubic graph using three colors. The four-color conjecture states that one can color a map using only four colors so that any adjacent countries have different colors. The four-color theorem was proven in 1976 with the use of computers. Later, in 1980, Hoyler proved edge-coloring problems are $N P$-complete. This means it is very unlikely that edge-coloring problems are able to be solved in polynomial-time. With this result, it shows us that we need to develop methods that will efficiently color graphs giving rise to one reason the problem in this thesis was pursued.

\subsection{Designs}

A balanced incomplete block design $(B I B D(v, k, \lambda))$ with parameters $(v, b, r, k, \lambda)$ is a pair $(\mathcal{V}, \mathcal{B})$ where $\mathcal{V}$ is a $v$-set of points and $\mathcal{B}$ is a collection of $b k$-subsets of $\mathcal{V}$, called blocks, such that each element of $\mathcal{V}$ is contained in exactly $r$ blocks and any pairs of points of $\mathcal{V}$ is contained in exactly $\lambda$ blocks. A BIBD is complete if it has no repeating blocks and contain $\left(\begin{array}{l}v \\ k\end{array}\right)$ blocks. We denote this as $\operatorname{BIBD}(v, k, \lambda)$ because the values for $b$ and $r$ can be calculated with only $v, k$, and $\lambda$.

To explain designs further we will use one of the most common examples in design theory; the Fano plane seen in Figure 1.4. Each line on this figure and the circle represent the seven blocks of the design. Our goal is to construct a $\operatorname{BIBD}(7,3,1)$ so let $\mathcal{V}=\{0,1,2,3,4,5,6\}$. The parameters of a $\operatorname{BIBD}(7,3,1)$ tell us we are going to make blocks of size 3, 3-subsets, and we will see any pair of points appear exactly once. Let 


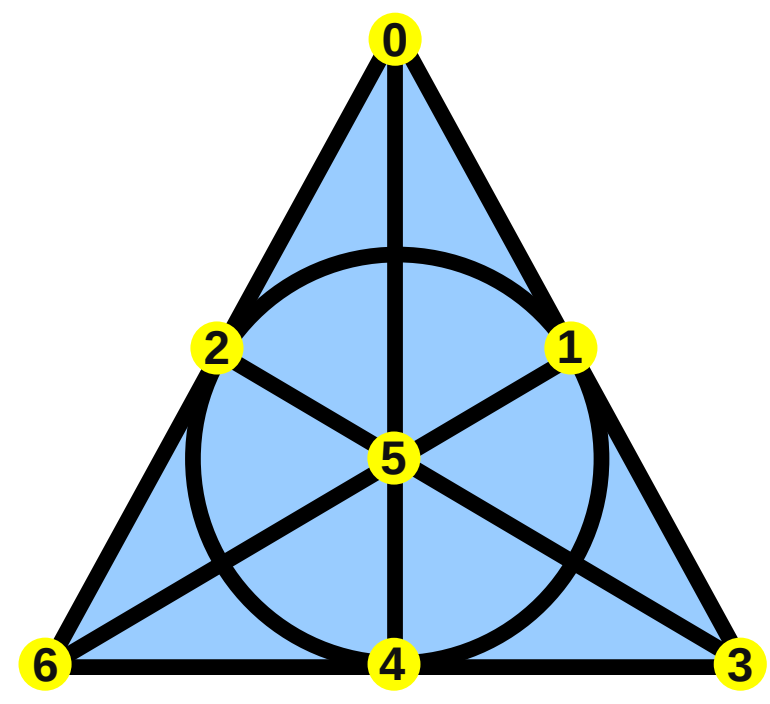

Figure 1.4: The Fano plane.

$B_{1}=\{0,1,3\}$ be the first block where $B_{1} \in \mathcal{B}$. From this we will develop a set of blocks cyclically from $B_{1}$ modulo 7 . Here is the set of seven blocks we develop from $B_{1}$.

$$
\{0,1,3\} \quad\{1,2,4\} \quad\{2,3,5\} \quad\{3,4,6\} \quad\{4,5,0\} \quad\{5,6,1\} \quad\{6,0,2\}
$$

These seven blocks make up $\mathcal{B}$ and so we have constructed a $\operatorname{BIBD}(7,3,1)$. We know we have constructed all the blocks because there is a formula for finding the number of blocks; $b=\frac{v(v-1)}{k(k-1)} \lambda$. In our case,

$$
b=\frac{v(v-1)}{k(k-1)} \lambda=\frac{7(6)}{3(2)} \cdot 1=7 .
$$

Due to the complexity of designs, this method of cyclically constructing designs does not always work. It is only because this design is cyclic that we may construct it in this fashion. Keep this in mind when we talk about designs being sufficient.

The numbers $v, b, r, k$, and $\lambda$ are parameters of the BIBD. A special type of BIBD called a triple system $(\mathrm{TS}(v, \lambda))$ is a $\mathrm{BIBD}$ where $k=3$. And a special type of triple system called Steiner triple system $(\operatorname{STS}(v))$ is a BIBD where $\lambda=1$ and $k=3$ or a triple system where $\lambda=1$. The necessary conditions under which a BIBD exists are given below.

Theorem 1.2. (Hanani, [28]) The necessary conditions for the existence of a $\operatorname{BIBD}(v, b, r, k, \lambda)$ are

1. $v r=b k$

2. $r(k-1)=\lambda(v-1)$ 
Though the first questions in design theory were contrived many centuries ago, our focus will begin in 1847 when Kirkman [31] dealt with the existence of $\operatorname{STS}(v)$ which exist for all $v \equiv 1,3(\bmod 6)$. Kirkman had a famous schoolgirl problem [32] that dealt with STS designs:

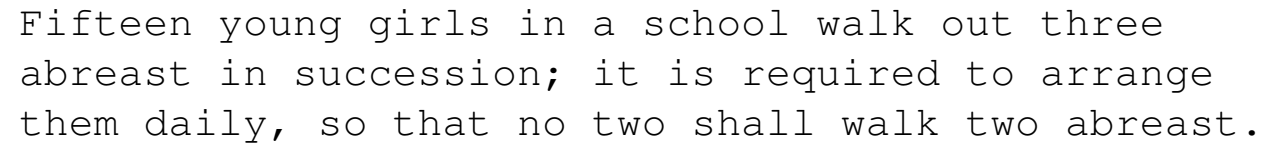

Because there are 15 young girls, $v=15$. When the girls walk three abreast, this means that we group the girls together in groups of size three. Thus we have an example of an $\operatorname{STS}(15)$ or a $\operatorname{BIBD}(15,3,1)$.

Significant contributions to this field include Euler in 1782, Kirkman in 1847, Moore in 1896, Bose in 1939, and Wilson in 1972. Hanani also contributed by determining the necessary and sufficient conditions of BIBDs with block size 4 and 5 with any $\lambda$ value. Later, he published a survey over 100 pages long, detailing the previous existence results for blocks of size 3, 4, and 5. He then extended the results to block size 6 and any $\lambda \geq 2$.

Design theory has numerous applications throughout many fields, but the general consensus is that one of the most useful applications of design theory is in setting up experimental designs. One such application can be summed up as making a consumer experiment where consumers try products and give responses to questionnaires about the products they try. All the products are alterations of one main concept such as fast food experiments where a group of 30 to 40 consumers try out different types of foods. Since it is a principal feature that all samples be evaluated the same number of times, a balanced incomplete block design contains this and more features making it ideal for this type of experiment. We also want to have pairs of samples to be evaluated the same number of times by some consumer. To do this, we just let the consumer represent a block of different samples. Since each sample occurs $r$ times and each pair of samples occurs $\lambda$ times, the experimental design is said to be a BIBD.

A large list of parameters for BIBDs are listed in [16] by Cochran and Cox. Since their designs were meant for agricultural applications which had limited resources (i.e. animals and field plots) the number of repetitions is limited. The number of repetitions that consumer experiments tend to have at least 30 to 40 . So the size of designs limits the usefulness with real world applications but with ever increasing technology, it does not seem infeasible that larger designs may be used in the near future.

Another type of design we will focus heavily on is the group divisible design or (GDD). A group divisible design of index $\lambda,(k, \lambda)-\operatorname{GDD}\left(m^{u}\right)$, is a triple $(\mathcal{V}, \mathcal{G}, \mathcal{B})$ where $\mathcal{V}$ is a finite set of $v=m u$ points, $\mathcal{G}$ is a partition of $\mathcal{V}$ into parts (groups) whose sizes lie in a set of positive integers, and $\mathcal{B}$ is a family of subsets (blocks) of $\mathcal{V}$ that satisfy the following.

1. If $B \in \mathcal{B}$, then $|B|=k$.

2. If two elements are in the same group, then this pair cannot be in any block.

3. $|\mathcal{G}|>1$. 
We use exponential notation to denote the type of the GDD. For example, a GDD having $u$ groups of size $m$ would be referred to as a $\operatorname{GDD}\left(m^{u}\right)$ as in Figure 1.5 . A pair of distinct points coming from two distinct groups of a GDD is called a transverse pair. A GDD is uniform if all groups have the same size and denoted as $k-\operatorname{GDD}\left(m^{u}\right)$.

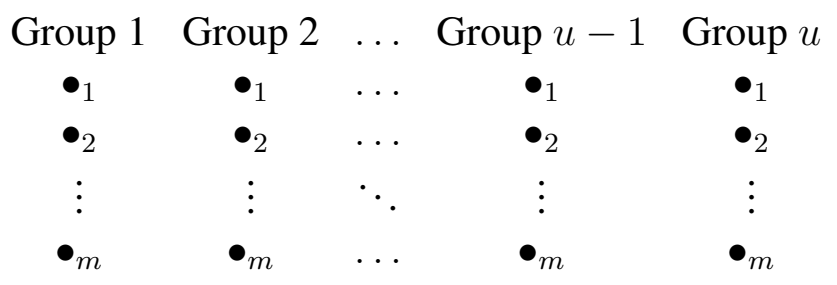

Figure 1.5: $k-\mathrm{GDD}\left(m^{u}\right)$

GDDs can be extended to BIBDs by making sure every pair of points occurs in $\lambda$ blocks. This method is similar to Wilson's Fundamental Construction [51]. As an example, consider a $4-\operatorname{GDD}\left(4^{4}\right)$ setup as in Figure 1.6. Our goal is to create a $\operatorname{BIBD}(16,4,1)$. We

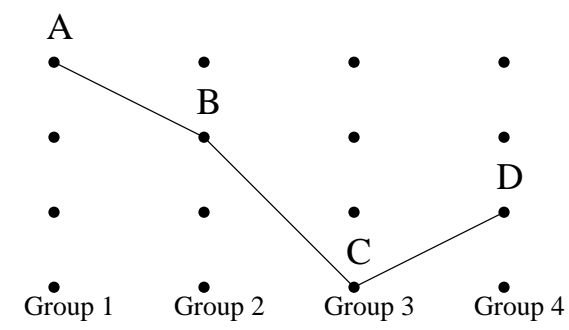

Figure 1.6: One block of a $4-\operatorname{GDD}\left(4^{4}\right)$

know this GDD exists because the necessary and sufficient conditions for the existence of a $(4, \lambda)-\operatorname{GDD}\left(m^{u}\right)$ were found by Zhu in [55]. They are given in the following theorem.

Theorem 1.3. The necessary and sufficient conditions for the existence of a $(4, \lambda)-$ $\operatorname{GDD}\left(m^{u}\right)$ are

1. $u \geq 4$,

2. $\lambda(u-1) m \equiv 0(\bmod 3)$, and

3. $\lambda u(u-1) m^{2} \equiv 0(\bmod 12)$,

with exception of $(m, u, \lambda) \in\{(2,4,1),(6,4,1)\}$, in which case no such GDD exists.

In a GDD, every transverse pair occurs in exactly $\lambda$ blocks. This means that if $\lambda=1$, every pair occurs in exactly one block or group. The only pairs that have not been accounted 
for are those that lie in the same group. We know by Hanani in [28] that the necessary conditions in Theorem 1.2 are sufficient for the following $v$ values with $k=4$ :

\begin{tabular}{rrr}
\hline $\mathrm{k}$ & $\lambda$ & Conditions for $v$ \\
\hline 4 & 1 & $1,4(\bmod 12)$ \\
4 & 2 & $1(\bmod 3)$ \\
4 & 3 & $0,1(\bmod 4)$ \\
4 & 6 & all \\
\hline
\end{tabular}

Consider the set of points in a group as the set of points from which we form a design. From these set of four points we can form a $\operatorname{BIBD}(4,4,1)$ design by the table above. Because we do this to each group, we add the blocks from each $\operatorname{BIBD}(4,4,1)$ on each group to the blocks we formed from the blocks of the GDD. This action of forming BIBDs on groups will be known as placing a BIBD on each group. This ensures that each pair among the groups has $\lambda=1$. Therefore, there exists a $\operatorname{BIBD}(16,4,1)$.

Non-uniform GDDs have also been studied.

Theorem 1.4. (Ge, Ling, [24] $) A 4-\operatorname{GDD}\left(4^{u} m^{1}\right)$ exists if and only if either $u=3$ and $m=4$, or $u \geq 6, u \equiv 0(\bmod 3)$ and $m \equiv 1(\bmod 3)$ with $1 \leq m \leq 2(u-1)$.

Theorem 1.5. (Zhu, [55]) The necessary conditions for the existence of a uniform $(5, \lambda)-$ $\operatorname{GDD}\left(m^{u}\right)$ are as follows.

1. $u \geq 5$,

2. $\lambda(u-1) m \equiv 0(\bmod 4)$, and

3. $\lambda u(u-1) m^{2} \equiv 0(\bmod 20)$.

Theorem 1.6. (Ge, Rees, Zhu, [25]) A $4-\mathrm{GDD}\left(1^{u} m^{1}\right)$ exists if and only if $u \geq 2 m+1$ and either $m, u+m \equiv 1$ or $4(\bmod 12)$ or $m, u+m \equiv 7$ or $10(\bmod 12)$.

Another class of GDDs that have been studied are (5, $\lambda)$-GDD.

Theorem 1.7. (Ge, Ling, [24]) The necessary conditions for the existence of a 5-GDD $\left(m^{u}\right)$ in Theorem 1.5 are also sufficient, except when $m^{u} \in\left\{2^{5}, 2^{11}, 3^{5}, 6^{5}\right\}$, and possibly where

1. $m^{u}=3^{45}, 3^{65}$;

2. $m \equiv 2,6,14,18(\bmod 20)$ and

(a) $m=2$ and $u \in\{15,35,71,75,95,111,115,195,215\}$;

(b) $m=6$ and $u \in\{15,35,75,95\}$;

(c) $m=18$ and $u \in\{11,15,71,111,115\}$;

(d) $m \in\{14,22,26,34,38,46,58,62\}$ and $u \in\{11,15,71,75,111,115\}$; 
(e) $m \in\{42,54\}$ or $m=2 \alpha$ with $\alpha \equiv 1,3,7,9(\bmod 10)$ and $33 \leq \alpha \leq 2443$, and $u=15$;

3. $m \equiv 10(\bmod 20)$ and

(a) $m=10$ and $u \in\{5,7,15,23,27,33,35,39,47\}$;

(b) $m=30$ and $u \in\{9,15\}$;

(c) $m=50$ and $u \in\{15,23,27\}$;

(d) $m=90$ and $u=23$;

(e) $m=10 \alpha, \alpha \equiv 1(\bmod 6), 7 \leq \alpha \leq 319$, and $u \in\{15,23\}$;

(f) $m=10 \beta, \beta \equiv 5(\bmod 6), 11 \leq \beta \leq 443$, and $u \in\{15,23\}$;

(g) $m=10 \gamma, \gamma \equiv 1(\bmod 6), 325 \leq \gamma \leq 487$, and $u=15$;

(h) $m=10 \delta, \delta \equiv 5(\bmod 6), 449 \leq \delta \leq 485$, and $u=15$;

Theorem 1.8. (Assaf, Bluskov, Greig, Shalaby, [11]) Let $\lambda \geq 2$. The necessary conditions for the existence of $(5, \lambda)-$ GDDs of type $m^{u}$ in Theorem 1.5 are also sufficient, except possibly when $\lambda=2, u=15$ and either $m=9 \operatorname{or} \operatorname{gcd}(m, 15)=1$.

Let $\mathcal{B}$ be a set of blocks in a GDD or BIBD. A parallel class or resolution class is a collection of blocks that partition the point-set of the design. A design is resolvable if the blocks of the design can be partitioned into parallel classes.

A resolvable balanced incomplete block design (RBIBD) is a balanced incomplete block design where the design is resolvable. The following are the necessary conditions for the existence of $\operatorname{RBIBD}(v, k, \lambda)$ :

1. $\lambda(v-1) \equiv 0(\bmod k-1)$,

2. $v \equiv 0(\bmod k)$.

From [22], these conditions are known to be sufficient for any $k$ and $\lambda$, if $v$ is large enough.

Theorem 1.9. [7, 36, 23]. An $\operatorname{RBIBD}(v, 5, \lambda)$ exists for $\lambda=1,2,4$, if any of the following conditions is satisfied:

1. $\lambda=1, v \equiv 5(\bmod 20)$ and $v \neq 45,185,225,345,465,645$;

2. $\lambda=2, v \equiv 5(\bmod 10)$ and $v \geq 50722395$;

3. $\lambda=4, v \equiv 0(\bmod 5)$ except for $v=10$ and possibly for $v=15,70,75,90,95,135,160,185,190,195$.

In the same manner, a resolvable GDD is a GDD where the blocks of the design can be partitioned into parallel classes, denoted as RGDD. 
Theorem 1.10. (Ge, Ling, [24]) The necessary conditions for the existence of a 4 $\operatorname{RGDD}\left(m^{u}\right)$, namely, $u \geq 4, m u \equiv 0(\bmod 4)$ and $m(u-1) \equiv 0(\bmod 3)$, are also sufficient except for $(m, u) \in\{(2,4),(2,10),(3,4),(6,4)\}$ and possibly excepting: $m=2$ and $u \in\{34,46,52,70,82,94,100,118,130,142,178,184,202,214,238,250,334,346\}$; $m=10$ and $u \in\{4,34,52,94\} ; m \in[14,454] \cup\{478,502,514,526,614,626,686\}$ and $u \in\{10,70,82\} ; m=6$ and $u \in\{6,54,68\} ; m=18$ and $u \in\{18,38,62\}$; $m=9$ and $u=44 ; m=12$ and $u=27 ; m=24$ and $u=23$; and $m=36$ and $u \in\{11,14,15,18,23\}$. 


\section{Chapter 2}

\section{Proper Edge Colorings of $\operatorname{BIBD}(v, k, \lambda)$}

Even though the problem is stated in graph theoretic terms, the focus of this thesis remains to be design theory. In this chapter we will give a multitude of examples of the problem at hand and an example of every construction we use in Chapters 3 and 4 . Pictorial representations will be used when appropriate.

\subsection{The Focus}

Before we start, make note that $\mathbb{Z}^{+}$represents the set of positive integers not including zero. We consider the following question

Question. Let $G=(V, E)$ be a graph and $\{1,2, \ldots, k\}$ be a set of colors. Can one decompose $\lambda$ copies of monochromatic $K_{v}$ into copies of $K_{k}$ such that each copy of $K_{k}$ contains at most one edge from each $K_{v}$ ?

A graph is monochromatic if all the edges of the graph are colored with the same color. Constructing panchromatic $K_{k}$ graphs from $\lambda$ monochromatic $K_{v}$ graphs is the same as properly coloring $K_{k}$ graphs using the edges of the monochromatic graphs. Note that $\lambda$ denotes the number of colors used as well as the number of monochromatic graphs.

In [29] Hurd and Sarvate translated this graph theory problem into a problem based on designs. Let us consider the graph $G=(V, E)$ being the complete graph on $v$ vertices such that $|V|=v$ and $|E|=\left(\begin{array}{l}v \\ 2\end{array}\right)=\frac{v(v-1)}{2}$. We copy this graph $\lambda$ times and give a unique color to each copy giving us $\lambda$ colors. We label all vertices in each copy of $K_{v}$ so as to distinguish which edge we remove based on the vertices that connect them. So each $K_{k}$ must have $k$ labeled vertices from $V$. Because we are trying to properly color a $K_{k}$ graph, we will remove at most one edge from each monochromatic $K_{v}$. For a $K_{k}$ to be properly colored, there need to be at least $\left(\begin{array}{l}k \\ 2\end{array}\right)$ unique colors so each edge in the $K_{k}$ can have a unique color. It follows that $\lambda \geq\left(\begin{array}{l}k \\ 2\end{array}\right)$ for us to have a chance of properly coloring each $K_{k}$. Let $b$ be the number of $K_{k}$ graphs we create through this decomposition of the $\lambda$ copies of $K_{v}$.

Suppose we represent the vertices of each $K_{k}=\left(V_{0}, E_{0}\right)$ as a set $\left\{v_{1}, v_{2}, \ldots, v_{k}\right\}$ of vertices where $v_{1}, v_{2}, \ldots, v_{k} \in V_{0} \subset V$. Because the graph is complete on $K_{k}$ it is easy to form the complete graph on $k$ vertices from the set $\left\{v_{1}, v_{2}, \ldots, v_{k}\right\}$. Each one of these sets 
will be of the same size $k$, and we shall denote each of these sets as $B_{i}$ such that $B_{i} \in \mathcal{B}$ for all $i=1,2, \ldots, b$. Because a particular edge appears once in each of the $\lambda$ copies of $K_{v}$, there are $\lambda$ copies of this edge, each with a different color. We must ensure the number of times a particular edge is seen among the $K_{k}$ graphs is $\lambda$. This is the same as confirming every pair of distinct vertices is in exactly $\lambda$ of the $B_{i}$. At this point, notice that each set, or as we will call them blocks, has identical size $k$ where each vertex is chosen from a set of $v$ vertices and we must make sure that the number of pairs we see among all the blocks is $\lambda$. This is identical to the definition of balanced incomplete block design. As such, rather than saying we will color the pairs of points in a design so as to create properly colored $K_{k}$ graphs from $K_{v}$ graphs, we will say we have a properly colored design. So our objective for this paper equates to showing we can properly color a $\operatorname{BIBD}(v, k, \lambda)$.

We want to decompose $\lambda$ copies of monochromatic $K_{v}$ into copies $K_{k}$ such that each copy of $K_{k}$ contains at most one edge from each $K_{v}$. A proper coloring, or proper edgecoloring, of a $\operatorname{BIBD}(v, k, \lambda)$ is an assignment of colors to the edges of a $K_{k}$ denoted by a block of the design (a block of the design denotes the $K_{k}$ graph) with the properties:

1. each edge in a $K_{k}$ graph generated from the points in a block receives a different color;

2. every edge is used exactly $\lambda$ times;

3. every color is used exactly once among the $\lambda$ copies of an edge.

In [29] Hurd and Sarvate discussed and solved the cases where $\lambda=m k(k-1) / 2$ for any $\operatorname{BIBD}(v, k, \lambda)$. They also found the sufficient conditions for the existence of a properly colored triple system $\operatorname{TS}(v, \lambda)$. The following theorems are some results found by Hurd and Survate in [29].

Theorem 2.1. The necessary conditions are sufficient for the existence of a $\operatorname{TS}(v, \lambda)$ which has a proper coloring.

Theorem 2.2. Suppose that there exists a $\operatorname{BIBD}(v, k, \lambda),(\mathcal{V}, \mathcal{B})$ with $\lambda=m k(k-1) / 2$, $m \in \mathbb{Z}^{+}$. Then the blocks of $\mathcal{B}$ can be properly colored with $\lambda$ colors so that no two edges in any block have the same color.

Theorem 2.3. (Hurd, Sarvate, [29]) If a $\operatorname{BIBD}(v, k, 1)$ exists, then for the index $\lambda \geq$ $k(k-1) / 2$ there exists a $\mathrm{BIBD}(v, k, \lambda)$ whose edges can be taken from $\lambda$ monochromatic copies of $K_{v}$ so that no two edges in a block have the same color.

As an example of properly coloring the block structure of a $K_{k}$ using the structure of a BIBD we will give a graphical representation of a proper coloring of three monochromatic $K_{7}$ into panchromatic $K_{3}$. Suppose we have three $K_{7}$ graphs, a blue, a red, and a green one. Let $\{0,1,3\}$ be a block of vertices that will make up one vertex set of a panchromatic $K_{3}$. Note all pairs and triples here are listed with braces to indicate the sets are unordered. Color edge $\{0,1\}$ red, edge $\{1,3\}$ green, and edge $\{0,3\}$ blue. To do this we remove the respective colored edges from the monochromatic $K_{7}$ graph to construct the panchromatic $K_{3}$. This is shown in Figure 2.1 with gray dashed lines indicating the edge has been removed from a $K_{7}$. Now we can repeat this block two more times to get three copies of 
this vertex set with different colors. The second block is based on the same vertex set, so color edge $\{0,1\}$ blue, edge $\{1,3\}$ red, and edge $\{0,3\}$ green. Again, we remove edges from the same monochromatic $K_{7}$ graphs to construct the panchromatic $K_{3}$. This is shown in Figure 2.2. We perform the same task for the last copy of this block by coloring edge $\{0,1\}$ green, edge $\{1,3\}$ blue, and edge $\{0,3\}$ red. This is shown in Figure 2.3 .
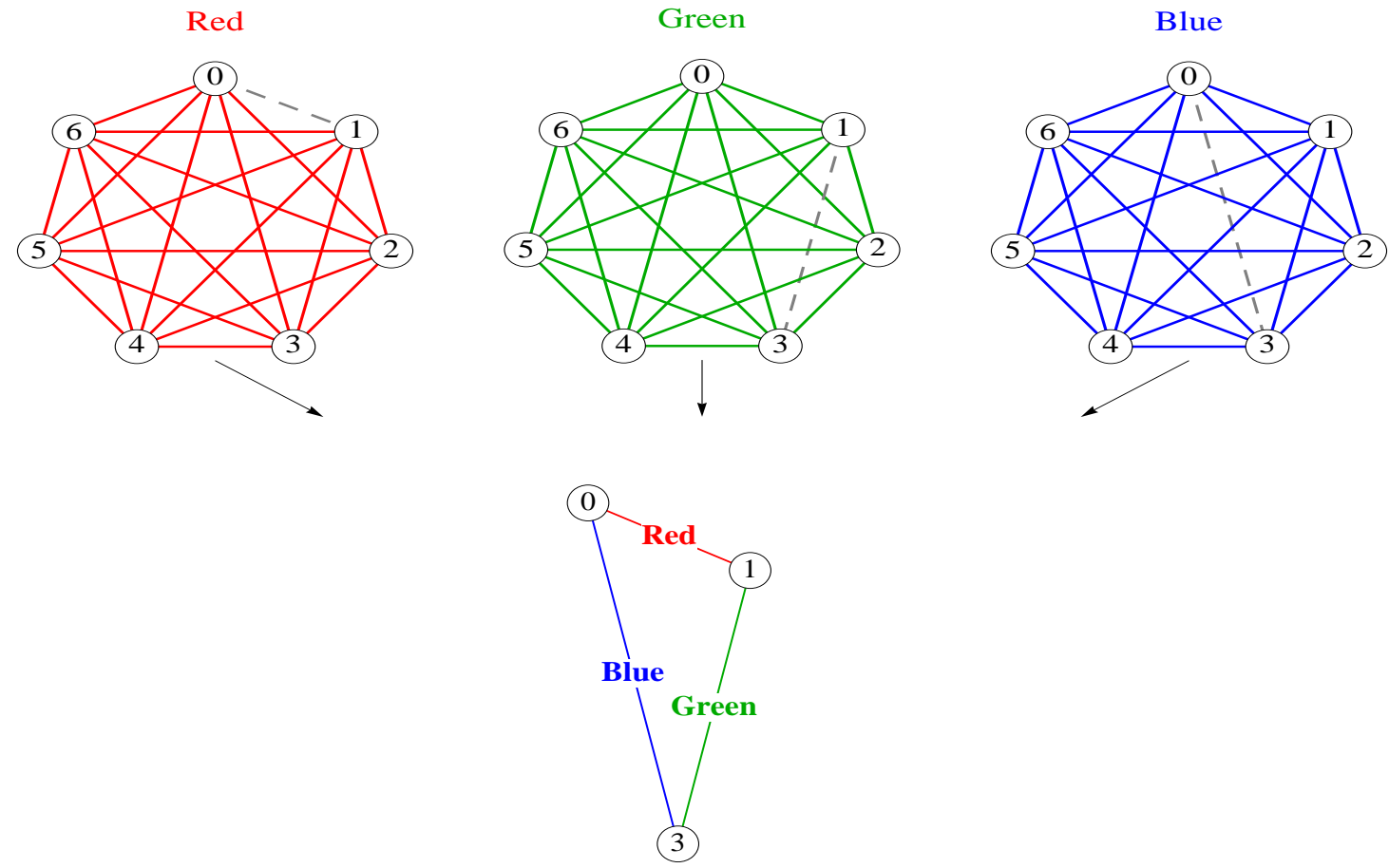

Figure 2.1: $1^{\text {st }}$ of three colored copies of a block from a $\operatorname{BIBD}(7,3,3)$

As one can see, we have 3 copies of a single $K_{3}$ from $\lambda=3$ copies of $K_{7}$. Because we can cyclically permute the block $\{0,1,3\}$ modulus 7 to get the remaining blocks the next block we would use is $\{1,2,4\}$. The same procedure just used on the block $\{0,1,3\}$ would be applied to this new block. This procedure will continue through each block until all the $K_{3}$ have been drawn with unique colorings on each edge, giving us a proper coloring.

Because the case where $k=3$ is completely solved in [29], we focused on the cases where $k=4$ and 5 . We will need to know the necessary and sufficient conditions from the existence of $\operatorname{a} \operatorname{BIBD}(v, 4, \lambda)$ and $\operatorname{a} \operatorname{BIBD}(v, 5, \lambda)$ if we are to form any link between properly colored graphs and coloring the incidence structure of a BIBD. 

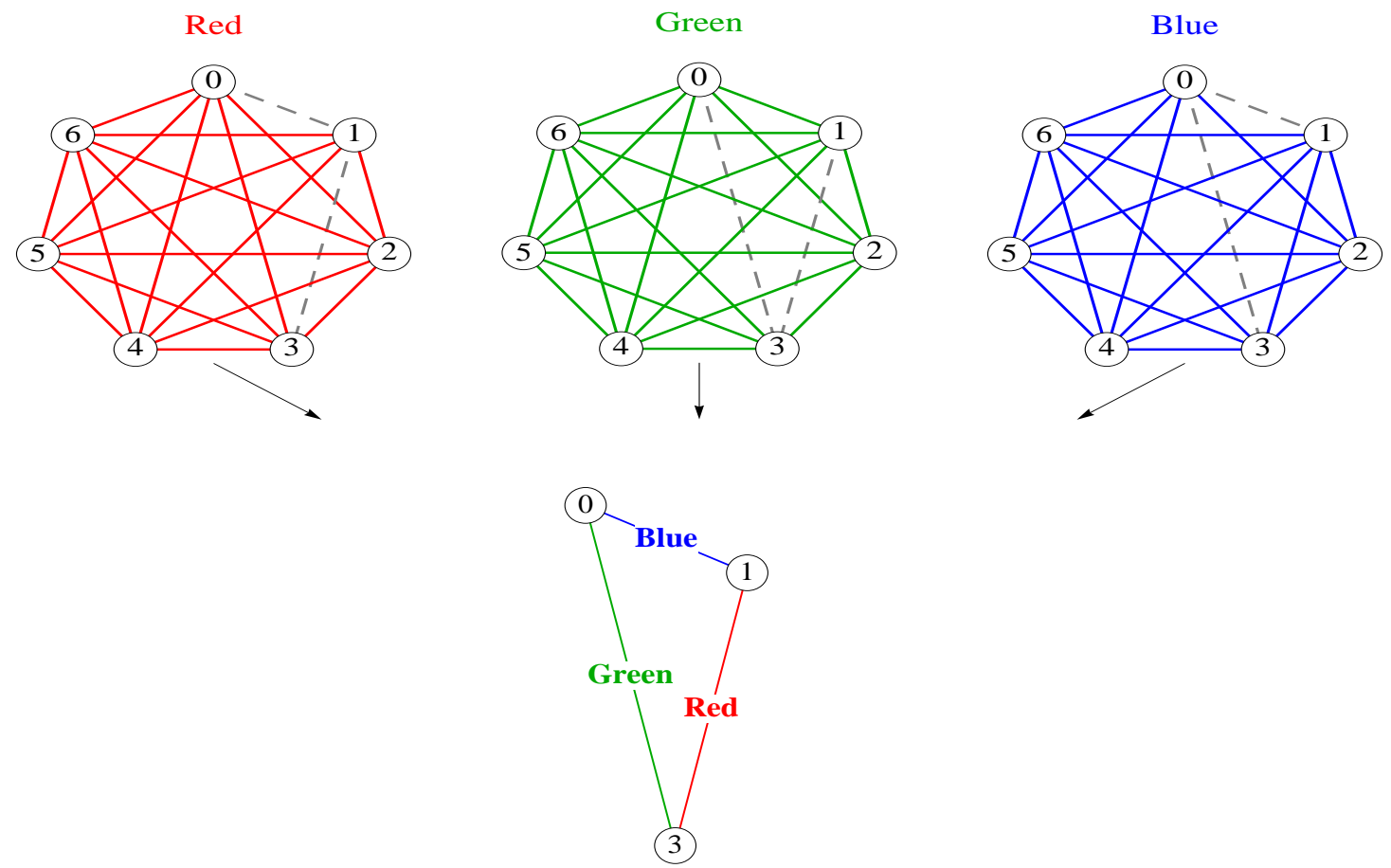

Figure 2.2: $2^{\text {nd }}$ of three colored copies of a block from a $\operatorname{BIBD}(7,3,3)$
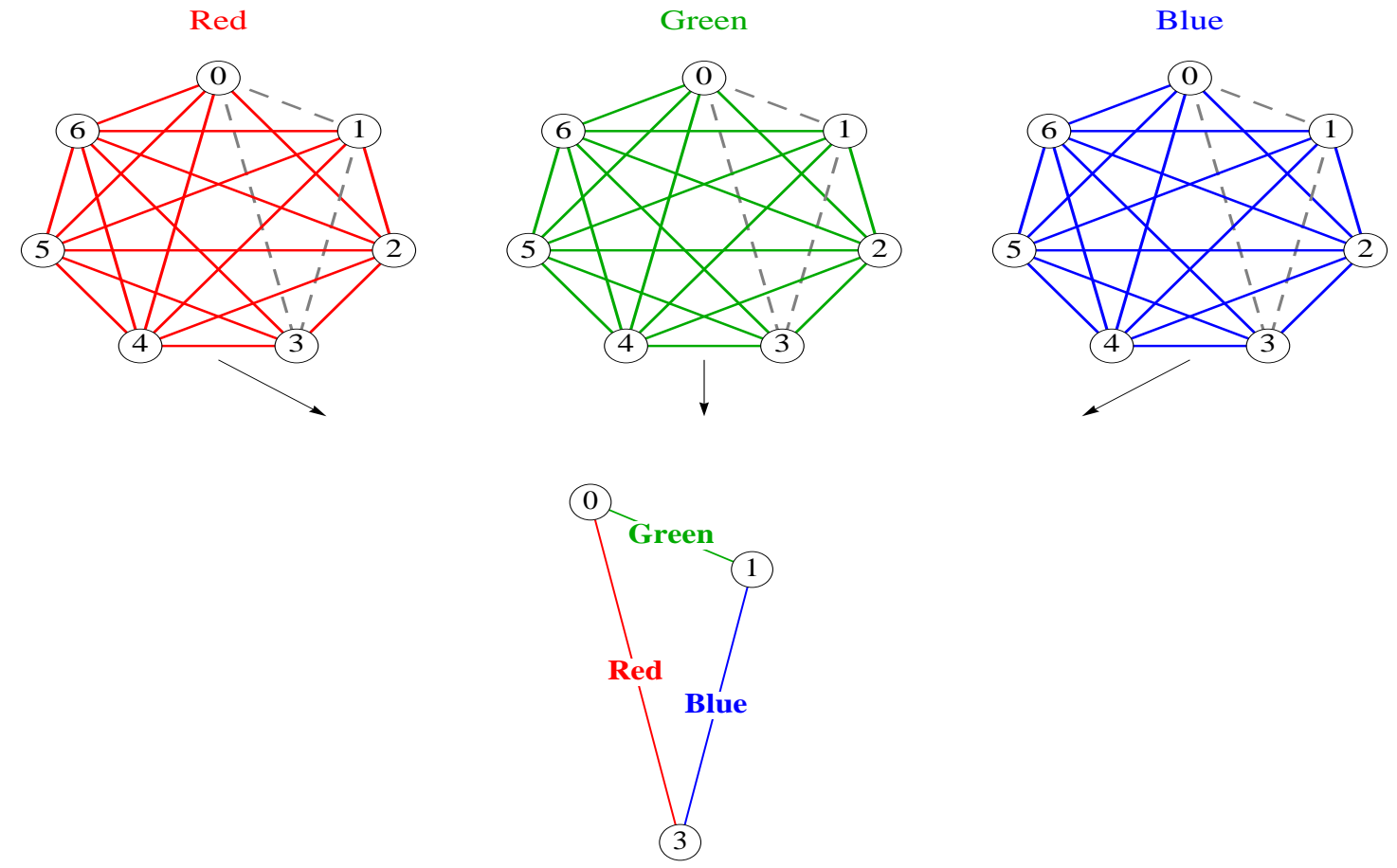

Figure 2.3: $3^{\text {rd }}$ of three colored copies of a block from a $\operatorname{BIBD}(7,3,3)$ 
The necessary and sufficient conditions for the existence of $\operatorname{B} \operatorname{BBD}(v, 4, \lambda)$ were obtained by Hanani in [28]. They are as follows:

$$
\begin{aligned}
& \text { If } \lambda \equiv 1,5(\bmod 6) \text {, then } v \equiv 1,4(\bmod 12) . \\
& \text { If } \lambda \equiv 2,4(\bmod 6) \text {, then } v \equiv 1(\bmod 3) . \\
& \text { If } \lambda \equiv 3(\bmod 6) \text {, then } v \equiv 0,1(\bmod 4) . \\
& \text { If } \lambda \equiv 0(\bmod 6) \text {, then } v \geq 4 .
\end{aligned}
$$

If $\lambda \equiv 1,5(\bmod 6)$, then we can properly color a $\operatorname{BIBD}(v, 4, \lambda)$ by applying Theorem 2.3 , and if $\lambda \equiv 0(\bmod 6)$, then we can properly color a $\operatorname{BIBD}(v, 4, \lambda)$ by applying Theorem 2.2. Therefore, in Chapter 3 we need only consider $\operatorname{BIBD}(v, 4, \lambda)$ with $\lambda \equiv 2,3,4$ $(\bmod 6)$.

The necessary and sufficient conditions for the existence of a $\operatorname{BIBD}(v, 5, \lambda)$ were obtained by Hanani in [28]. They are as follows:

$$
\begin{aligned}
& \text { If } \lambda \equiv 1,3,5,7,9,11,13,15,17,19(\bmod 20) \text {, then } v \equiv 1,5(\bmod 20) . \\
& \text { If } \lambda \equiv 2,6,14,18(\bmod 20) \text {, then } v \equiv 1,5(\bmod 10) . \\
& \text { If } \lambda \equiv 4,8,12,16(\bmod 20) \text {, then } v \equiv 0,1(\bmod 5) . \\
& \text { If } \lambda \equiv 10(\bmod 20), \text { then } v(\bmod 2) . \\
& \text { If } \lambda \equiv 0(\bmod 20) \text {, then } v \geq 5 .
\end{aligned}
$$

If $\lambda \equiv 1,3,5,7,9,11,13,15,17,19(\bmod 20)$, then we can properly color a $\operatorname{BIBD}(v, 5, \lambda)$ by applying Theorem 2.3, and if $\lambda \equiv 0,10(\bmod 20)$, then we can properly color a $\operatorname{BIBD}(v, 5, \lambda)$ by applying Theorem 2.2. Therefore, in Chapter 4 we need only consider $\operatorname{BIBD}(v, 5, \lambda)$ with $\lambda \equiv 2,4,6,8,12,14,16,18(\bmod 20)$.

Our main result, based on the above cases, is as follows. We prove the following theorems later.

Theorem. There is a proper edge coloring for every $\operatorname{BIBD}(v, 4, \lambda)$ if $\lambda \geq 6$.

Theorem. There is a proper edge coloring for every $\operatorname{BIBD}(v, 5, \lambda)$ if $\lambda \geq 10$ except possibly when $\lambda=2 k, k \geq 5, v \equiv 15,35,75,95(\bmod 100)$ and $\lambda=14,18$.

\subsection{Examples of Direct Constructions}

The methods we will use in this section will be described in full detail later in this thesis. The incidence matrix of a design is a $(0,1)$-matrix where the rows are indexed by the $v$ points and the columns are indexed by the $b$ blocks. In this matrix, each entry receives a 1 if the point lies in a block and 0 otherwise. This incidence structure has several useful properties. The sum of entries down each column is $k$ and the sum of the entries across 


\begin{tabular}{c|ccccccc|} 
& $B_{1}$ & $B_{2}$ & $B_{3}$ & $B_{4}$ & $B_{5}$ & $B_{6}$ & $B_{7}$ \\
\hline 0 & 1 & 0 & 0 & 0 & 1 & 0 & 1 \\
1 & 1 & 1 & 0 & 0 & 0 & 1 & 0 \\
2 & 0 & 1 & 1 & 0 & 0 & 0 & 1 \\
3 & 1 & 0 & 1 & 1 & 0 & 0 & 0 \\
4 & 0 & 1 & 0 & 1 & 1 & 0 & 0 \\
5 & 0 & 0 & 1 & 0 & 1 & 1 & 0 \\
6 & 0 & 0 & 0 & 1 & 0 & 1 & 1 \\
\hline
\end{tabular}

Figure 2.4: Incidence matrix of a $\operatorname{BIBD}(7,3,1)$

each row is $r$ which is the repetition number or the number of blocks a point will appear in. As an example we will use the $\operatorname{BIBD}(7,3,1)$ we constructed in Section 1.2 . Figure 2.4 depicts the incidence matrix for a $\operatorname{BIBD}(7,3,1)$.

Using the incidence matrix in Figure 2.4 we can form a more useful structure known as the edge-incidence matrix. An edge-incidence matrix is a $(0,1)$-matrix where the rows are indexed by all possible pairs from $\mathcal{V}$ and the columns are indexed by the $b$ blocks of the design. The matrix has a 1 in entry $(i, j)$ if pair $i$ is contained in block $j$, and an entry of 0 otherwise. A couple special properties of this matrix are that the sum of entries across each row is $\lambda$ and the sum of entries down each column is $\left(\begin{array}{l}k \\ 2\end{array}\right)$. Figure 2.5 depicts the edge-incidence matrix of the previous incidence matrix of a $\operatorname{BIBD}(7,3,1)$.

\begin{tabular}{l|ccccccc|} 
& $B_{1}$ & $B_{2}$ & $B_{3}$ & $B_{4}$ & $B_{5}$ & $B_{6}$ & $B_{7}$ \\
\hline$\{0,1\}$ & 1 & 0 & 0 & 0 & 0 & 0 & 0 \\
$\{1,2\}$ & 0 & 1 & 0 & 0 & 0 & 0 & 0 \\
$\{2,3\}$ & 0 & 0 & 1 & 0 & 0 & 0 & 0 \\
$\{3,4\}$ & 0 & 0 & 0 & 1 & 0 & 0 & 0 \\
$\{4,5\}$ & 0 & 0 & 0 & 0 & 1 & 0 & 0 \\
$\{5,6\}$ & 0 & 0 & 0 & 0 & 0 & 1 & 0 \\
$\{6,0\}$ & 0 & 0 & 0 & 0 & 0 & 0 & 1 \\
\hline$\{0,2\}$ & 0 & 0 & 0 & 0 & 0 & 0 & 1 \\
$\{1,3\}$ & 1 & 0 & 0 & 0 & 0 & 0 & 0 \\
$\{2,4\}$ & 0 & 1 & 0 & 0 & 0 & 0 & 0 \\
$\{3,5\}$ & 0 & 0 & 1 & 0 & 0 & 0 & 0 \\
$\{4,6\}$ & 0 & 0 & 0 & 1 & 0 & 0 & 0 \\
$\{5,0\}$ & 0 & 0 & 0 & 0 & 1 & 0 & 0 \\
$\{6,1\}$ & 0 & 0 & 0 & 0 & 0 & 1 & 0 \\
\hline$\{0,3\}$ & 1 & 0 & 0 & 0 & 0 & 0 & 0 \\
$\{1,4\}$ & 0 & 1 & 0 & 0 & 0 & 0 & 0 \\
$\{2,5\}$ & 0 & 0 & 1 & 0 & 0 & 0 & 0 \\
$\{3,6\}$ & 0 & 0 & 0 & 1 & 0 & 0 & 0 \\
$\{4,0\}$ & 0 & 0 & 0 & 0 & 1 & 0 & 0 \\
$\{5,1\}$ & 0 & 0 & 0 & 0 & 0 & 1 & 0 \\
$\{6,2\}$ & 0 & 0 & 0 & 0 & 0 & 0 & 1 \\
\hline
\end{tabular}

Figure 2.5: Edge-incidence matrix of a $\operatorname{BIBD}(7,3,1)$ 
To simplify our results we will use circulant matrices. A circulant matrix is an $n \times n$ square matrix, in which each row (except the first) is obtained from the preceding row by shifting the elements cyclically one column to the right. Figure 2.6 is a $7 \times 7$ circulant matrix whose first row is [1000000].

\begin{tabular}{|l|l|l|l|l|l|l|}
\hline 1 & 0 & 0 & 0 & 0 & 0 & 0 \\
\hline 0 & 1 & 0 & 0 & 0 & 0 & 0 \\
\hline 0 & 0 & 1 & 0 & 0 & 0 & 0 \\
\hline 0 & 0 & 0 & 1 & 0 & 0 & 0 \\
\hline 0 & 0 & 0 & 0 & 1 & 0 & 0 \\
\hline 0 & 0 & 0 & 0 & 0 & 1 & 0 \\
\hline 0 & 0 & 0 & 0 & 0 & 0 & 1 \\
\hline
\end{tabular}

Figure 2.6: A $7 \times 7$ circulant matrix.

An edge-coloring for a $\operatorname{BIBD}(v, k, \lambda)(\mathcal{V}, \mathcal{B})$ can be described by providing an edgecoloring incidence matrix. An edge-coloring incidence matrix is an edge-incidence matrix that has been given a coloring to each pair of points in the design. In the example from the beginning of this section we color each pair of points in a convenient pattern. It should be noted that there does not need to be one of each color in each column. We will use the colors from the set $\left\{c_{1}, c_{2}, c_{3}\right\}$ to color Figure 2.7 .

\begin{tabular}{c|ccccccc|} 
& $B_{1}$ & $B_{2}$ & $B_{3}$ & $B_{4}$ & $B_{5}$ & $B_{6}$ & $B_{7}$ \\
\hline$\{0,1\}$ & $c_{1}$ & 0 & 0 & 0 & 0 & 0 & 0 \\
$\{1,2\}$ & 0 & $c_{1}$ & 0 & 0 & 0 & 0 & 0 \\
$\{2,3\}$ & 0 & 0 & $c_{1}$ & 0 & 0 & 0 & 0 \\
$\{3,4\}$ & 0 & 0 & 0 & $c_{1}$ & 0 & 0 & 0 \\
$\{4,5\}$ & 0 & 0 & 0 & 0 & $c_{1}$ & 0 & 0 \\
$\{5,6\}$ & 0 & 0 & 0 & 0 & 0 & $c_{1}$ & 0 \\
$\{6,0\}$ & 0 & 0 & 0 & 0 & 0 & 0 & $c_{1}$ \\
\hline$\{0,2\}$ & 0 & 0 & 0 & 0 & 0 & 0 & $c_{2}$ \\
$\{1,3\}$ & $c_{2}$ & 0 & 0 & 0 & 0 & 0 & 0 \\
$\{2,4\}$ & 0 & $c_{2}$ & 0 & 0 & 0 & 0 & 0 \\
$\{3,5\}$ & 0 & 0 & $c_{2}$ & 0 & 0 & 0 & 0 \\
$\{4,6\}$ & 0 & 0 & 0 & $c_{2}$ & 0 & 0 & 0 \\
$\{5,0\}$ & 0 & 0 & 0 & 0 & $c_{2}$ & 0 & 0 \\
$\{6,1\}$ & 0 & 0 & 0 & 0 & 0 & $c_{2}$ & 0 \\
\hline$\{0,3\}$ & $c_{3}$ & 0 & 0 & 0 & 0 & 0 & 0 \\
$\{1,4\}$ & 0 & $c_{3}$ & 0 & 0 & 0 & 0 & 0 \\
$\{2,5\}$ & 0 & 0 & $c_{3}$ & 0 & 0 & 0 & 0 \\
$\{3,6\}$ & 0 & 0 & 0 & $c_{3}$ & 0 & 0 & 0 \\
$\{4,0\}$ & 0 & 0 & 0 & 0 & $c_{3}$ & 0 & 0 \\
$\{5,1\}$ & 0 & 0 & 0 & 0 & 0 & $c_{3}$ & 0 \\
$\{6,2\}$ & 0 & 0 & 0 & 0 & 0 & 0 & $c_{3}$ \\
\hline
\end{tabular}

Figure 2.7: Edge-colored incidence matrix of a $\operatorname{BIBD}(7,3,1)$ 
In general, this is the edge-colored matrix $M$ defined by

$$
M=M[\{x, y\}, B]= \begin{cases}c_{i} & \text { if }\{x, y\} \in B \text { has color } c_{i}, \\ 0 & \text { if }\{x, y\} \notin B\end{cases}
$$

where $c_{1}, c_{2}, \ldots, c_{\lambda}$ are the colors for all pairs $\{x, y\} \in\left(\begin{array}{l}\mathcal{V} \\ 2\end{array}\right)$ and $B \in \mathcal{B}$. Note that $\left(\begin{array}{l}\mathcal{V} \\ 2\end{array}\right)$ is the set of all pairs of $\mathcal{V}$. So the Figure 2.7 could be represented as

$$
\begin{array}{|c|}
\hline\left(c_{1}\right) I \\
\hline\left(c_{2}\right) A^{6} \\
\hline\left(c_{3}\right) I \\
\hline
\end{array}
$$

where $I$ is the $7 \times 7$ identity matrix and $A$ is the $7 \times 7$ circulant matrix whose first row is [0100000].

As an example, our goal is to properly color a $\operatorname{BIBD}(5,4, \lambda)$. We form the edgeincidence matrix for a $\operatorname{BIBD}(5,4,3)$ as in Figure 2.8. Each block represents the six edges

\begin{tabular}{c|ccccc|} 
& $B_{1}$ & $B_{2}$ & $B_{3}$ & $B_{4}$ & $B_{5}$ \\
\hline$\{1,2\}$ & 0 & 0 & 1 & 1 & 1 \\
$\{2,3\}$ & 1 & 0 & 0 & 1 & 1 \\
$\{3,4\}$ & 1 & 1 & 0 & 0 & 1 \\
$\{4,5\}$ & 1 & 1 & 1 & 0 & 0 \\
$\{5,1\}$ & 0 & 1 & 1 & 1 & 0 \\
\hline$\{1,3\}$ & 0 & 1 & 0 & 1 & 1 \\
$\{2,4\}$ & 1 & 0 & 1 & 0 & 1 \\
$\{3,5\}$ & 1 & 1 & 0 & 1 & 0 \\
$\{4,1\}$ & 0 & 1 & 1 & 0 & 1 \\
$\{5,2\}$ & 1 & 0 & 1 & 1 & 0 \\
\hline
\end{tabular}

Figure 2.8: Edge-incidence matrix for a $\operatorname{BIBD}(5,4,3)$

of a unique $K_{4}$. The notation $\{1,2\}$ represents the edge between vertex 1 and vertex 2 in the graph. To color this design, we replace each " 1 " with an entry $c_{i}$ which represents the color $i$ for $i=1,2, \ldots, \lambda$. Our matrix must have the property that each entry $c_{i}$ appears exactly once in every row and column. We can color the first 5 edges of the blocks in the $\operatorname{BIBD}(5,4,3)$ using 3 colors, and we can color the last 5 edges of the $\operatorname{BIBD}(5,4,3)$ using 3 colors to get the following edge-coloring incidence matrix using 6 colors in Figure 2.9.

Let $A$ be the $5 \times 5$ circulant matrix whose first row is [01000]. Then we can represent this edge-coloring incidence matrix in terms of $A$ as follows.

$$
\begin{array}{|c|}
\hline\left(c_{1}\right) A^{2}+\left(c_{2}\right) A^{3}+\left(c_{3}\right) A^{4} \\
\hline\left(c_{4}\right) A+\left(c_{5}\right) A^{3}+\left(c_{6}\right) A^{4} \\
\hline
\end{array}
$$




\begin{tabular}{c|ccccc|} 
& $B_{1}$ & $B_{2}$ & $B_{3}$ & $B_{4}$ & $B_{5}$ \\
\hline$\{1,2\}$ & 0 & 0 & $c_{1}$ & $c_{2}$ & $c_{3}$ \\
$\{2,3\}$ & $c_{3}$ & 0 & 0 & $c_{1}$ & $c_{2}$ \\
$\{3,4\}$ & $c_{2}$ & $c_{3}$ & 0 & 0 & $c_{1}$ \\
$\{4,5\}$ & $c_{1}$ & $c_{2}$ & $c_{3}$ & 0 & 0 \\
$\{5,1\}$ & 0 & $c_{1}$ & $c_{2}$ & $c_{3}$ & 0 \\
\hline$\{1,3\}$ & 0 & $c_{4}$ & 0 & $c_{5}$ & $c_{6}$ \\
$\{2,4\}$ & $c_{6}$ & 0 & $c_{4}$ & 0 & $c_{5}$ \\
$\{3,5\}$ & $c_{5}$ & $c_{6}$ & 0 & $c_{4}$ & 0 \\
$\{4,1\}$ & 0 & $c_{5}$ & $c_{6}$ & 0 & $c_{4}$ \\
$\{5,2\}$ & $c_{4}$ & 0 & $c_{5}$ & $c_{6}$ & 0 \\
\hline
\end{tabular}

Figure 2.9: Edge-Coloring Incidence Matrix for a $\operatorname{BIBD}(5,4,3)$

Next we form a properly colored $\operatorname{BIBD}(5,4, \lambda)$. To form a properly colored $\operatorname{BIBD}(5,4, \lambda)$ with $\lambda=3 s, s \geq 2$, we simply repeat the blocks of the $\operatorname{BIBD}(5,4,3)$ $s$ times and follow the same coloring scheme with different colors. Define $M_{i}$ as the following sub-matrix. Note that all subscripts are computed $(\bmod \lambda)$ where we identify $c_{0}$ with $c_{\lambda}$.

$$
M_{i}=\frac{\left(c_{1+3 i}\right) A^{2}+\left(c_{2+3 i}\right) A^{3}+\left(c_{3+3 i}\right) A^{4}}{\left(c_{4+3 i}\right) A+\left(c_{5+3 i}\right) A^{3}+\left(c_{6+3 i}\right) A^{4}}
$$

Then the edge-coloring incidence matrix of a properly colored $\operatorname{BIBD}(5,4, \lambda)$ with $\lambda=3 s$, $s \geq 2$ is given by

$$
M=\begin{array}{|l|l|l|l|}
\hline M_{0} & M_{1} & \cdots & M_{k-1} \\
\hline
\end{array}
$$

The matrix $M$ has the property that every color $c_{1}, c_{2}, \ldots, c_{\lambda}=c_{0}$ is seen exactly once in each row, and every color is seen at most once in every column. Therefore, $M$ is an edge-coloring incidence matrix of a properly colored $\operatorname{BIBD}(5,4, \lambda)$. We state this result as a lemma.

Lemma 2.4. There exists a properly colored $\operatorname{BIBD}(5,4, \lambda)$ where $\lambda=3 s, s \in \mathbb{Z}^{+}, s \geq 2$.

As an example of this lemma, we provide the edge-coloring incidence matrix of a properly colored $\operatorname{BIBD}(5,4,9)$ in Figure 2.10.

At this time, let us bring focus to why we want to repeat each block of a $\operatorname{BIBD}(v, k, \lambda)$ enough times to get $\lambda \geq\left(\begin{array}{c}k \\ 2\end{array}\right)$. In each $K_{k}$, there are $\left(\begin{array}{c}k \\ 2\end{array}\right)$ colors. Because each row of the matrix represents an edge and we need at least each edge to appear in $\left(\begin{array}{l}k \\ 2\end{array}\right)$ colors, we must 


\begin{tabular}{c|ccccc|ccccc|ccccc|} 
& $B_{1}$ & $B_{2}$ & $B_{3}$ & $B_{4}$ & $B_{5}$ & $B_{6}$ & $B_{7}$ & $B_{8}$ & $B_{9}$ & $B_{10}$ & $B_{11}$ & $B_{12}$ & $B_{13}$ & $B_{14}$ & $B_{15}$ \\
\hline$\{1,2\}$ & 0 & 0 & $c_{1}$ & $c_{2}$ & $c_{3}$ & 0 & 0 & $c_{4}$ & $c_{5}$ & $c_{6}$ & 0 & 0 & $c_{7}$ & $c_{8}$ & $c_{9}$ \\
$\{2,3\}$ & $c_{3}$ & 0 & 0 & $c_{1}$ & $c_{2}$ & $c_{6}$ & 0 & 0 & $c_{4}$ & $c_{5}$ & $c_{9}$ & 0 & 0 & $c_{7}$ & $c_{8}$ \\
$\{3,4\}$ & $c_{2}$ & $c_{3}$ & 0 & 0 & $c_{1}$ & $c_{5}$ & $c_{6}$ & 0 & 0 & $c_{4}$ & $c_{8}$ & $c_{9}$ & 0 & 0 & $c_{7}$ \\
$\{4,5\}$ & $c_{1}$ & $c_{2}$ & $c_{3}$ & 0 & 0 & $c_{4}$ & $c_{5}$ & $c_{6}$ & 0 & 0 & $c_{7}$ & $c_{8}$ & $c_{9}$ & 0 & 0 \\
$\{5,1\}$ & 0 & $c_{1}$ & $c_{2}$ & $c_{3}$ & 0 & 0 & $c_{4}$ & $c_{5}$ & $c_{6}$ & 0 & 0 & $c_{7}$ & $c_{8}$ & $c_{9}$ & 0 \\
\hline$\{1,3\}$ & 0 & $c_{4}$ & 0 & $c_{5}$ & $c_{6}$ & 0 & $c_{7}$ & 0 & $c_{8}$ & $c_{9}$ & 0 & $c_{1}$ & 0 & $c_{2}$ & $c_{3}$ \\
$\{2,4\}$ & $c_{6}$ & 0 & $c_{4}$ & 0 & $c_{5}$ & $c_{9}$ & 0 & $c_{6}$ & 0 & $c_{8}$ & $c_{3}$ & 0 & $c_{1}$ & 0 & $c_{2}$ \\
$\{3,5\}$ & $c_{5}$ & $c_{6}$ & 0 & $c_{4}$ & 0 & $c_{8}$ & $c_{9}$ & 0 & $c_{7}$ & 0 & $c_{2}$ & $c_{3}$ & 0 & $c_{1}$ & 0 \\
$\{4,1\}$ & 0 & $c_{5}$ & $c_{6}$ & 0 & $c_{4}$ & 0 & $c_{8}$ & $c_{9}$ & 0 & $c_{7}$ & 0 & $c_{2}$ & $c_{3}$ & 0 & $c_{1}$ \\
$\{5,2\}$ & $c_{4}$ & 0 & $c_{5}$ & $c_{6}$ & 0 & $c_{7}$ & 0 & $c_{8}$ & $c_{9}$ & 0 & $c_{1}$ & 0 & $c_{2}$ & $c_{3}$ & 0 \\
\hline
\end{tabular}

Figure 2.10: Edge-Coloring Incidence Matrix of a properly colored $\operatorname{BIBD}(5,4,9)$.

have $\left(\begin{array}{l}k \\ 2\end{array}\right)$ unique colors in each row. But the number of entries in each row is $\lambda$. Thus $\lambda \geq\left(\begin{array}{c}k \\ 2\end{array}\right)$.

The following example illustrates one of the main ideas used in the construction of a properly colored $\operatorname{BIBD}(v, 4, \lambda)$ design.

Example 2.5. A properly colored $\operatorname{BIBD}(17,4,9)$.

Because a $\operatorname{BIBD}(17,4,1)$ does not exist we cannot apply Theorem 2.3. It is known by Theorem 1.3 that a $(4,1)-\operatorname{GDD}\left(4^{4}\right)(\mathcal{V}, \mathcal{B}, \mathcal{G})$ exists where $\mathcal{V}$ is the point set, $\mathcal{B}$ is the set of blocks, and $\mathcal{G}$ is the set of groups $G_{1}, G_{2}, G_{3}, G_{4}$. We will construct a $\operatorname{BIBD}(17,4,9)$ design on $\mathcal{V} \cup\{\infty\}$. Let $A, B, C, D \in \mathcal{V}$. For each block $\{A, B, C, D\}$ of the GDD, make 9 copies of the block. Let $K_{4}$ represent this block as in Figure 2.11. We can see that given a block $\{A, B, C, D\}$ implies there is an edge between each point in the block.

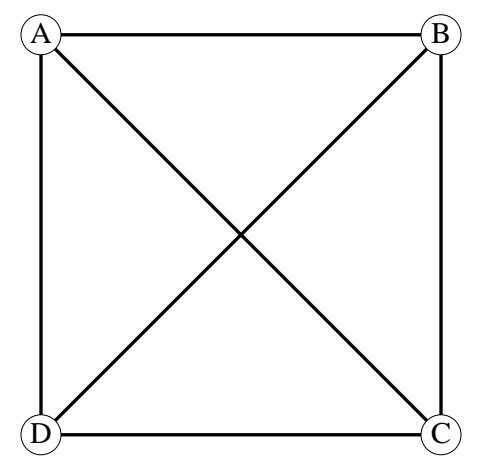

Figure 2.11: $K_{4}$ 
Our goal is to color each edge of this block a different color using the colors $c_{i} \in$ $\left\{c_{1}, \ldots, c_{9}\right\}$. Each corresponding edge in the 9 copies of the block must also be a different color. So we form a $6 \times 9$ matrix. The rows of the matrix will be indexed by the 6 edges of $K_{4}$, and the columns will be indexed by the 9 copies of our block. The entries will represent the color of each edge. We must see every color $c_{1}, \ldots, c_{9}$ exactly once in each row and at most once in any column. The matrix for our 9 colored copies of $\{A, B, C, D\}$ is shown in Figure 2.12.

\begin{tabular}{l|ccccccccc}
\multicolumn{1}{c}{} & $B_{1}$ & $B_{2}$ & $B_{3}$ & $B_{4}$ & $B_{5}$ & $B_{6}$ & $B_{7}$ & $B_{8}$ & $B_{9}$ \\
$\{A, B\}$ & $c_{1}$ & $c_{2}$ & $c_{3}$ & $c_{4}$ & $c_{5}$ & $c_{6}$ & $c_{7}$ & $c_{8}$ & $c_{9}$ \\
$\{A, C\}$ & $c_{9}$ & $c_{1}$ & $c_{2}$ & $c_{3}$ & $c_{4}$ & $c_{5}$ & $c_{6}$ & $c_{7}$ & $c_{8}$ \\
$\{A, D\}$ & $c_{8}$ & $c_{9}$ & $c_{1}$ & $c_{2}$ & $c_{3}$ & $c_{4}$ & $c_{5}$ & $c_{6}$ & $c_{7}$ \\
$\{B, C\}$ & $c_{7}$ & $c_{8}$ & $c_{9}$ & $c_{1}$ & $c_{2}$ & $c_{3}$ & $c_{4}$ & $c_{5}$ & $c_{6}$ \\
$\{B, D\}$ & $c_{6}$ & $c_{7}$ & $c_{8}$ & $c_{9}$ & $c_{1}$ & $c_{2}$ & $c_{3}$ & $c_{4}$ & $c_{5}$ \\
$\{C, D\}$ & $c_{5}$ & $c_{6}$ & $c_{7}$ & $c_{8}$ & $c_{9}$ & $c_{1}$ & $c_{2}$ & $c_{3}$ & $c_{4}$ \\
\cline { 2 - 8 }
\end{tabular}

Figure 2.12: First 6 rows of a $\operatorname{LS}(9)$

Notice this matrix is simply the first 6 rows of an $\operatorname{LS}(9)$, a Latin square of side 9. Because an $\operatorname{LS}(9)$ is a $9 \times 9$ matrix and we only need 6 rows, we can always properly color the blocks of a GDD. We do this for each block of the GDD.

The only pairs that have not been covered are pairs that lie within the groups and pairs that involve $\{\infty\}$ as in Figure 2.13. These pairs need to be covered 9 times. So con-

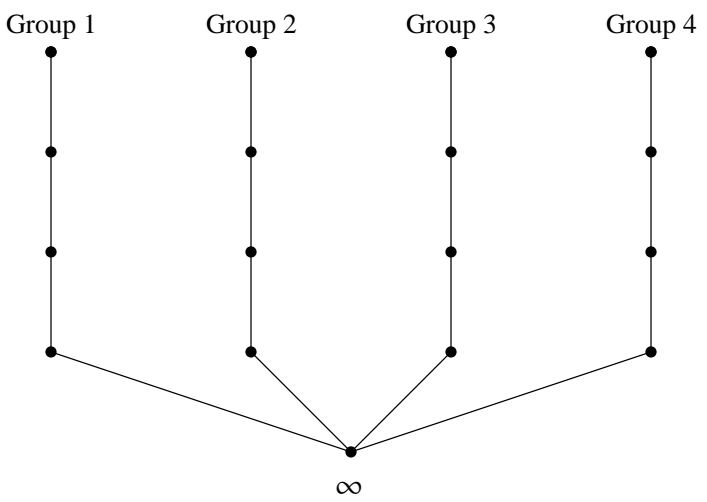

Figure 2.13: Coloring within groups of a GDD

struct a properly colored $\operatorname{BIBD}(5,4,9)$ on each group $G_{i} \cup\{\infty\}$ for $i=1,2,3,4$. This exists by Lemma 2.4. Now we have colored every block of the GDD and every group. We know through the use of Wilson's Construction from [51] that our GDD will yield a 
$\operatorname{BIBD}(17,4,3)$, which we can copy 3 times to generate a properly colored $\operatorname{BIBD}(17,4,9)$.

Example 2.6. A properly colored $\operatorname{BIBD}(24,4,9)$.

Our goal here is to describe the process of using 4-RGDDs when $k=4$. As we defined before, a group divisible design is resolvable when we can separate the blocks into parallel classes. This allows us to add points to parallel classes and still hold the properties of a design and the RGDD. Let $P_{i}$ for $i=1,2,3,4,5$ denote the parallel classes in the $4-\operatorname{RGDD}\left(5^{4}\right)$ which exists by Theorem 1.10 . Also let $\left\{\infty_{1}, \infty_{2}, \infty_{3}, \infty_{4}\right\}$ be four new points as in Figure 2.14 .

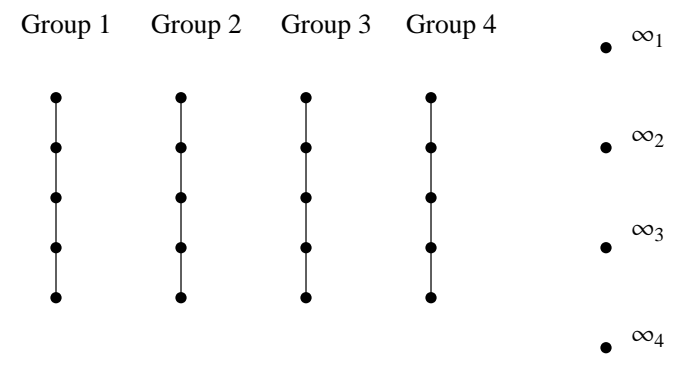

Figure 2.14: A 4-RGDD $\left(5^{4}\right)$

For each of the first four parallel classes, $P_{i}$, we place a properly colored $\operatorname{BIBD}(5,4,9)$ on each block plus $\infty_{i}$ just as in Figure 2.15. This ensures that each pair including exactly one of the $\infty_{i}$ points is covered exactly once.

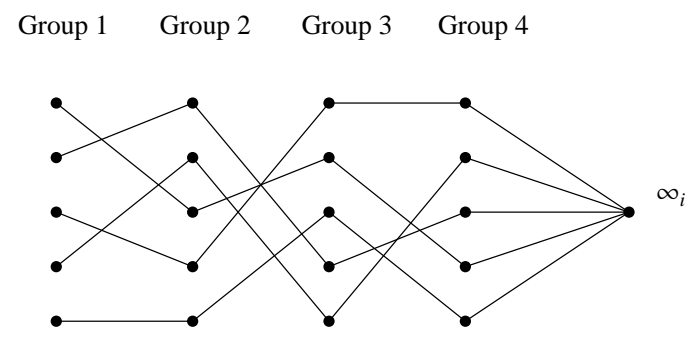

Figure 2.15: $P_{i} \cup \infty_{i}$ on a $4-\operatorname{RGDD}\left(5^{4}\right)$

Since there are only five parallel classes, color each block $\{A, B, C, D\}$ from parallel class $P_{5}$ as follows. We form a $6 \times 9$ matrix. The rows of the matrix will be indexed by the 6 edges of $K_{4}$, and the columns will be indexed by the 9 copies of our block. The entries will represent the color of each edge. We must see every color $c_{1}, \ldots, c_{9}$ exactly once in each row, and we must see each color at most once in any column. Coloring $P_{5}$ is the same as coloring the blocks of a 5-GDD as in Example 2.5

Notice this matrix is simply the first 6 rows of an $\operatorname{LS}(9)$. Because an $\operatorname{LS}(9)$ is a $9 \times 9$ matrix and we only need 6 rows, we can always properly color the blocks of the RGDD. We do this for each block of $P_{5}$ in the RGDD. The only pairs remaining are those that lie 
within the groups and those that lie within $\left\{\infty_{1}, \infty_{2}, \infty_{3}, \infty_{4}\right\}$. We know we can properly color a $\operatorname{BIBD}(5,4,9)$. Therefore, we can properly color a $\operatorname{BIBD}(24,4,9)$.

Now we shift our focus to constructions for properly colored $\operatorname{BIBD}(v, 5, \lambda)$.

Example 2.7. A properly colored $\operatorname{BIBD}(25,5,20)$.

The process shown in Example 2.5 can be extended to 5-GDDs using a similar method. Thus, it does not take much to realize that we could also use $(5,2)-$ GDDs. The construction is very similar, as well, to how we construct a properly colored $\mathrm{B} \operatorname{IBD}(v, k, \lambda)$ using a $5-$ GDD. The difference is that each pair of points occurs 2 times within the blocks because we will use a $(5,2)-$ GDD and this will force $\lambda$ to be at least 20. It is known by Theorem 1.7 that a $(5,2)-\operatorname{GDD}\left(5^{5}\right)(\mathcal{V}, \mathcal{B}, \mathcal{G})$ exists where $\mathcal{V}$ is the point set, $\mathcal{B}$ is the set of blocks, and $\mathcal{G}$ is the set of groups.

Consider two distinct color sets $C=\left\{c_{1}, \ldots, c_{10}\right\}$ and $D=\left\{d_{1}, \ldots, d_{10}\right\}$ that we will use to properly color each block. Our goal is to construct a properly colored edge-coloring incidence matrix made up of the blocks of the $(5,2)-\operatorname{GDD}\left(5^{5}\right)$ as shown in Figure 2.16 . To construct this matrix we replace the first " 1 " in each row with a $C$ and the second " 1 " by $D$. Since each block is being repeated 10 times we will use every color in the sets $C$ and $D$ in the edge-coloring incidence matrix.

$\{x, y\} \quad$\begin{tabular}{ccccccc}
$B_{1}$ & $B_{2}$ & $\ldots$ & $B^{\prime}$ & $\ldots$ & $B_{b-1}$ & $B_{b}$ \\
\hline 0 & $C$ & $\ldots$ & $D$ & $\ldots$ & 0 & 0 \\
$C$ & 0 & $\ldots$ & 0 & $\ldots$ & $D$ & 0 \\
0 & 0 & $\ldots$ & $C$ & $\ldots$ & 0 & $D$ \\
$\vdots$ & $\vdots$ & $\ddots$ & $\vdots$ & $\ddots$ & $\vdots$ & $\vdots$ \\
0 & 0 & $\ldots$ & $C$ & $\ldots$ & $D$ & 0 \\
$\vdots$ & $\vdots$ & $\ddots$ & $\vdots$ & $\ddots$ & $\vdots$ & $\vdots$ \\
$C$ & 0 & $\ldots$ & $D$ & $\ldots$ & 0 & 0 \\
\hline
\end{tabular}

Figure 2.16: Edge-coloring incidence matrix for $(5,2)-$ GDD

This gives us 20 unique colors in each row of this matrix. Now we must make sure there are 10 unique colors in each column. We will permute each of the color sets to make sure that if we were to construct an LS(10) with the non-zero entries of the 10 copies of the blocks we will have unique entries down each column. This can be seen in Figure 2.18

Consider only the block $B^{\prime}$. Let $t_{C}$ represent the number of times we use the color set $C$ and $t_{D}$ represent the number of times we use the color set $D$. From Figure 2.17, $t_{C}=8$ and $t_{D}=2$. Figure 2.18 depicts the nonzero entries within the ten copies of $B^{\prime}$.

We do repeat this for every block which gives use a unique color down each column. Thus we can properly color the blocks of the $(5,2)-\operatorname{GDD}\left(5^{5}\right)$. The only pairs left are those that lie within the groups. These pairs need to be covered 20 times. So construct a properly colored $\operatorname{BIBD}(5,5,20)$ on each group. This exists by Theorem 2.3 . Now we have colored every block of the GDD and every group. We know through the use of Wilson's Constructions in [51] that our $\operatorname{GDD}$ will yield a $\operatorname{BIBD}(25,5,20)$ which we properly colored. 


$$
\{x, y\} \quad \begin{array}{ccccccc}
B_{1} & B_{2} & \ldots & B^{\prime} & \ldots & B_{b-1} & B_{b} \\
\hline 0 & C_{1} & \ldots & D_{1} & \ldots & 0 & 0 \\
C_{1} & 0 & \ldots & 0 & \ldots & D_{1} & 0 \\
0 & 0 & \ldots & C_{1} & \ldots & 0 & D_{1} \\
\vdots & \vdots & \ddots & \vdots & \ddots & \vdots & \vdots \\
0 & 0 & \ldots & C_{2} & \ldots & D_{2} & 0 \\
\vdots & \vdots & \ddots & \vdots & \ddots & \vdots & \vdots \\
C_{10} & 0 & \ldots & D_{2} & \ldots & 0 & 0 \\
\hline
\end{array}
$$

Figure 2.17: Edge-coloring incidence matrix for $(5,2)-$ GDD with cyclic shifting

\begin{tabular}{|cccccccccc}
$B_{1}^{\prime}$ & $B_{2}^{\prime}$ & $B_{3}^{\prime}$ & $B_{4}^{\prime}$ & $B_{5}^{\prime}$ & $B_{6}^{\prime}$ & $B_{7}^{\prime}$ & $B_{8}^{\prime}$ & $B_{9}^{\prime}$ & $B_{10}^{\prime}$ \\
\hline$d_{1}$ & $d_{2}$ & $d_{3}$ & $d_{4}$ & $d_{5}$ & $d_{6}$ & $d_{7}$ & $d_{8}$ & $d_{9}$ & $d_{10}$ \\
$c_{1}$ & $c_{2}$ & $c_{3}$ & $c_{4}$ & $c_{5}$ & $c_{6}$ & $c_{7}$ & $c_{8}$ & $c_{9}$ & $c_{10}$ \\
$c_{10}$ & $c_{1}$ & $c_{2}$ & $c_{3}$ & $c_{4}$ & $c_{5}$ & $c_{6}$ & $c_{7}$ & $c_{8}$ & $c_{9}$ \\
$c_{9}$ & $c_{10}$ & $c_{1}$ & $c_{2}$ & $c_{3}$ & $c_{4}$ & $c_{5}$ & $c_{6}$ & $c_{7}$ & $c_{8}$ \\
$c_{8}$ & $c_{9}$ & $c_{10}$ & $c_{1}$ & $c_{2}$ & $c_{3}$ & $c_{4}$ & $c_{5}$ & $c_{6}$ & $c_{7}$ \\
$c_{7}$ & $c_{8}$ & $c_{9}$ & $c_{10}$ & $c_{1}$ & $c_{2}$ & $c_{3}$ & $c_{4}$ & $c_{5}$ & $c_{6}$ \\
$c_{6}$ & $c_{7}$ & $c_{8}$ & $c_{9}$ & $c_{10}$ & $c_{1}$ & $c_{2}$ & $c_{3}$ & $c_{4}$ & $c_{5}$ \\
$c_{5}$ & $c_{6}$ & $c_{7}$ & $c_{8}$ & $c_{9}$ & $c_{10}$ & $c_{1}$ & $c_{2}$ & $c_{3}$ & $c_{4}$ \\
$c_{4}$ & $c_{5}$ & $c_{6}$ & $c_{7}$ & $c_{8}$ & $c_{9}$ & $c_{10}$ & $c_{1}$ & $c_{2}$ & $c_{3}$ \\
$c_{3}$ & $c_{4}$ & $c_{5}$ & $c_{6}$ & $c_{7}$ & $c_{8}$ & $c_{9}$ & $c_{10}$ & $c_{1}$ & $c_{2}$ \\
$d_{10}$ & $d_{1}$ & $d_{2}$ & $d_{3}$ & $d_{4}$ & $d_{5}$ & $d_{6}$ & $d_{7}$ & $d_{8}$ & $d_{9}$ \\
\hline
\end{tabular}

Figure 2.18: $\mathrm{LS}(10)$ of 10 copies of block $B^{\prime}$

Example 2.8. A properly colored $\operatorname{BIBD}(31,5,12)$

This very useful construction is used in Chapter 4 where we use RBIBDs. We know there exists an $\operatorname{RBIBD}(25,5,1)$ by Theorem 1.9 . This also tells us we have 6 parallel classes which we denote by $P_{i}$ for $i=1, \ldots, 6$. Let $\left\{\infty_{1}, \infty_{2}, \infty_{3}, \infty_{4}, \infty_{5}, \infty_{6}\right\}$ be six new points. Now place a properly colored $\operatorname{BIBD}(6,5,12)$ on each block of $P_{i} \cup\left\{\infty_{i}\right\}$. To color this we use the same method found in Example 2.6. Normally, we have not covered the pairs that lie in the parallel classes $P_{i}$ for $i>6$ but we used all the parallel classes already. Now the only remaining pairs are of the form $\left\{\left\{\infty_{i}, \infty_{j}\right\}: i, j \in\{1, \ldots, 6\}\right\}$, so we place a properly colored $\operatorname{BIBD}(6,5,12)$ on the set of points $\left\{\infty_{1}, \ldots, \infty_{6}\right\}$. Because we can properly color every pair and we added six points, we can properly color a $\operatorname{BIBD}(31,5,12)$.

Example 2.9. A properly colored $\operatorname{BIBD}(471,5, \lambda)$ where $\lambda=2 k, k \in \mathbb{Z}^{+}, k \geq 5$.

Let $\lambda=2 k, k \in \mathbb{Z}^{+}, k \geq 5$. This construction is very similar to Example 2.6 since we are going to be using $4-\mathrm{RGDDs}$ as well. Let $P_{i}$ for $i=1, \ldots, t$ denote the parallel classes 
in the $4-\operatorname{RGDD}\left(30^{12}\right)$ which exists by Theorem 1.10 . Because this design is resolvable it has parallel classes. In fact, 110 parallel classes. We need to have 110 new points which we will denote as $\left\{\infty_{1}, \infty_{2}, \ldots, \infty_{110}\right\}$. We set up all the point just like as in Figure 2.14. For each of the 110 we place a properly colored $\operatorname{BIBD}(5,5, \lambda)$ on each block plus $\{\infty\}$ just as in Figure 2.15. This ensures that each pair including exactly one of the $\infty_{i}$ points is covered exactly once.

At this time, we note that we only have 470 points. So we add one last point called $\{\infty\}$ as in Figure 2.13. The only pairs remaining are those that lie within the groups with $\{\infty\}$ and those that lie in $\left\{\infty_{1}, \ldots, \infty_{110}\right\}$ with $\{\infty\}$. We can properly color a $\operatorname{BIBD}(31,5, \lambda)$ by Lemma 4.2. If $v=111=10(11)+1$, there exists a $5-\operatorname{GDD}\left(10^{11}\right)$ by Lemma 1.7. There also exists a properly colored $\operatorname{BIBD}(11,5, \lambda)$. Thus, we can properly color a $\operatorname{BIBD}(111,5, \lambda)$ by the same reasoning applied to Example 2.5. This reasoning will be stated as Lemma 4.18 later in the paper. Therefore, we can properly color a $\operatorname{BIBD}(471,5, \lambda)$. We state this result as a lemma.

Lemma 2.10. There exists a properly colored $\operatorname{BIBD}(471,5, \lambda)$ where $\lambda=2 k, k \in \mathbb{Z}^{+}, k \geq$ 5 .

Suppose there exists a $\operatorname{BIBD}(v, k, \lambda)$ where in each row and column of the edgecoloring incidence matrix there is a unique color from the color set $\{1,2, \ldots, \lambda\}$. We will examine what conclusions we can make if $\lambda<\left(\begin{array}{l}k \\ 2\end{array}\right)$.

Definition 2.11. We define a semi-properly colored $\operatorname{BIBD}$ is a $\operatorname{BIBD}(v, k, \lambda)$ with the following properties:

1. $\lambda<\left(\begin{array}{l}k \\ 2\end{array}\right)$

2. The set of color indices in each row of the edge-coloring incidence matrix forms the set $\{0,1, \ldots, \lambda-1\}(\bmod \lambda)$.

3. The set of colors in each column contain $\left(\begin{array}{l}k \\ 2\end{array}\right)$ unique colors

Theorem 2.12. If there exists a $\operatorname{BIBD}(v, k, \lambda)$ and it is semi-properly colored, then $a$ $\operatorname{BIBD}(v, k, m \lambda)$ is properly colored for $m \lambda \geq\left(\begin{array}{c}k \\ 2\end{array}\right), m \in \mathbb{Z}^{+}$.

Proof: Suppose we have the edge-colored incidence matrix of a semi-properly colored $\operatorname{BIBD}(v, k, \lambda)$. To build a properly colored $\operatorname{BIBD}(v, k, m \lambda)$, we first copy each block of the $\operatorname{BIBD}(v, k, \lambda) m$ times. This is equivalent to concatenating $m-1$ copies of the edgecolored incidence matrix of the semi-properly colored $\operatorname{BIBD}(v, k, \lambda)$. For $t=1,2, \ldots, m-$ 1 , add $t \lambda(\bmod m \lambda)$ to each color index, in the $t^{t h}$ copy of the matrix. Then the resulting matrix has the property that the $m \lambda$ entries are unique in each row. It is clear that we still have $\left(\begin{array}{l}k \\ 2\end{array}\right)$ unique colors in each column. Therefore, the new matrix is the edge-coloring incidence matrix of a properly colored $\operatorname{BIBD}(v, k, m \lambda)$.

From now on, when we prove a direct construction for a properly colored BIBD we will almost exclusively use Theorem 2.12 to simplify the proof. Examples of the use of this theorem can be seen in Section 3.1 and 4.1. 


\section{Chapter 3}

\section{Proper Edge Colorings With Block Size 4}

Understanding how to use the constructions in this chapter is imperative to following the reasoning in the main theorem. Before we get to the generalized constructions we must first show there exists properly colored $\operatorname{BIBD}(v, 4, \lambda)$ for small $v$.

As a standard among people in design theory, the use of $\infty$ is that of an extra point rather than a number. In some fields in discrete mathematics, $\infty$ is used as both a number and a point as in projective geometry and elliptic curves.

\subsection{Direct Constructions With Block Size 4}

Lemma 3.1. There exists a properly edge-colored $\operatorname{BIBD}(9,4, \lambda)$ design where $\lambda=3 k, k \in$ $\mathbb{Z}^{+}, k \geq 2$.

Proof: As in Lemma 2.4 we form an edge-coloring incidence matrix for a $\operatorname{BIBD}(9,4,3)$ as follows. Figure 3.1 represents the edge-coloring incidence matrix for the $\operatorname{BIBD}(9,4,3)$.

Let $A$ be the $9 \times 9$ circulant matrix whose first row is [010000000]. Then the edge coloring incidence matrix of a $\operatorname{BIBD}(9,4,3)$ using 6 colors is given below.

$$
M_{i}=\begin{array}{|c|c|}
\hline\left(c_{2+3 i}\right) I+\left(c_{1+3 i}\right) A^{8} & \left(c_{3+3 i}\right) I \\
\hline\left(c_{3+3 i}\right) A^{8} & \left(c_{2+3 i}\right) A^{3}+\left(c_{1+3 i}\right) A^{8} \\
\hline\left(c_{4+3 i}\right) I+\left(c_{5+3 i}\right) A^{7} & \left(c_{6+3 i}\right) A^{5} \\
\hline\left(c_{6+3 i}\right) I & \left(c_{4+3 i}\right) I+\left(c_{5+3 i}\right) A^{3} \\
\hline
\end{array}
$$

Since $M_{i}$ is semi-properly colored, by applying Theorem 2.12 we can properly color a $\operatorname{BIBD}(9,4, \lambda)$. 


\begin{tabular}{|c|c|c|c|c|c|c|c|c|c|c|c|c|c|c|c|c|c|c|}
\hline & $B_{1}$ & $B_{2}$ & $B_{3}$ & $B_{4}$ & $B_{5}$ & $B_{6}$ & $B_{7}$ & $B_{8}$ & $B_{9}$ & $B_{10}$ & $B_{11}$ & $B_{12}$ & $B_{13}$ & $B_{14}$ & $B_{15}$ & $B_{16}$ & $B_{17}$ & $B_{18}$ \\
\hline$\{0,1\}$ & $c_{2}$ & 0 & 0 & 0 & 0 & 0 & 0 & 0 & $c_{1}$ & $c_{3}$ & 0 & 0 & 0 & 0 & 0 & 0 & 0 & 0 \\
\hline$\{1,2\}$ & $c_{1}$ & $c_{2}$ & 0 & 0 & 0 & 0 & 0 & 0 & 0 & 0 & $c_{3}$ & 0 & 0 & 0 & 0 & 0 & 0 & 0 \\
\hline$\{2,3\}$ & 0 & $c_{1}$ & $c_{2}$ & 0 & 0 & 0 & 0 & 0 & 0 & 0 & 0 & $c_{3}$ & 0 & 0 & 0 & 0 & 0 & 0 \\
\hline$\{3,4\}$ & 0 & 0 & $c_{1}$ & $c_{2}$ & 0 & 0 & 0 & 0 & 0 & 0 & 0 & 0 & $c_{3}$ & 0 & 0 & 0 & 0 & 0 \\
\hline$\{4,5\}$ & 0 & 0 & 0 & $c_{1}$ & $c_{2}$ & 0 & 0 & 0 & 0 & 0 & 0 & 0 & 0 & $c_{3}$ & 0 & 0 & 0 & 0 \\
\hline$\{5,6\}$ & 0 & 0 & 0 & 0 & $c_{1}$ & $c_{2}$ & 0 & 0 & 0 & 0 & 0 & 0 & 0 & 0 & $c_{3}$ & 0 & 0 & 0 \\
\hline$\{6,7\}$ & 0 & 0 & 0 & 0 & 0 & $c_{1}$ & $c_{2}$ & 0 & 0 & 0 & 0 & 0 & 0 & 0 & 0 & $c_{3}$ & 0 & 0 \\
\hline$\{7,8\}$ & 0 & 0 & 0 & 0 & 0 & 0 & $c_{1}$ & $c_{2}$ & 0 & 0 & 0 & 0 & 0 & 0 & 0 & 0 & $c_{3}$ & 0 \\
\hline$\{8,0\}$ & 0 & 0 & 0 & 0 & 0 & 0 & 0 & $c_{1}$ & $c_{2}$ & 0 & 0 & 0 & 0 & 0 & 0 & 0 & 0 & $c_{3}$ \\
\hline$\{0,3\}$ & 0 & 0 & 0 & 0 & 0 & 0 & 0 & 0 & $c_{3}$ & 0 & 0 & 0 & $c_{2}$ & 0 & 0 & 0 & 0 & $c_{1}$ \\
\hline$\{1,4\}$ & $c_{3}$ & 0 & 0 & 0 & 0 & 0 & 0 & 0 & 0 & $c_{1}$ & 0 & 0 & 0 & $c_{2}$ & 0 & 0 & 0 & 0 \\
\hline$\{2,5\}$ & 0 & $c_{3}$ & 0 & 0 & 0 & 0 & 0 & 0 & 0 & 0 & $c_{1}$ & 0 & 0 & 0 & $c_{2}$ & 0 & 0 & 0 \\
\hline$\{3,6\}$ & 0 & 0 & $c_{3}$ & 0 & 0 & 0 & 0 & 0 & 0 & 0 & 0 & $c_{1}$ & 0 & 0 & 0 & $c_{2}$ & 0 & 0 \\
\hline$\{4,7\}$ & 0 & 0 & 0 & $c_{3}$ & 0 & 0 & 0 & 0 & 0 & 0 & 0 & 0 & $c_{1}$ & 0 & 0 & 0 & $c_{2}$ & 0 \\
\hline$\{5,8\}$ & 0 & 0 & 0 & 0 & $c_{3}$ & 0 & 0 & 0 & 0 & 0 & 0 & 0 & 0 & $c_{1}$ & 0 & 0 & 0 & $c_{2}$ \\
\hline$\{6,0\}$ & 0 & 0 & 0 & 0 & 0 & $c_{3}$ & 0 & 0 & 0 & $c_{2}$ & 0 & 0 & 0 & 0 & $c_{1}$ & 0 & 0 & 0 \\
\hline$\{7,1\}$ & 0 & 0 & 0 & 0 & 0 & 0 & $c_{3}$ & 0 & 0 & 0 & $c_{2}$ & 0 & 0 & 0 & 0 & $c_{1}$ & 0 & 0 \\
\hline$\{8,2\}$ & 0 & 0 & 0 & 0 & 0 & 0 & 0 & $c_{3}$ & 0 & 0 & 0 & $c_{2}$ & 0 & 0 & 0 & 0 & $c_{1}$ & 0 \\
\hline$\{0,2\}$ & $c_{4}$ & 0 & 0 & 0 & 0 & 0 & 0 & $c_{5}$ & 0 & 0 & 0 & 0 & 0 & 0 & $c_{6}$ & 0 & 0 & 0 \\
\hline$\{1,3\}$ & 0 & $c_{4}$ & 0 & 0 & 0 & 0 & 0 & 0 & $c_{5}$ & 0 & 0 & 0 & 0 & 0 & 0 & $c_{6}$ & 0 & 0 \\
\hline$\{2,4\}$ & $c_{5}$ & 0 & $c_{4}$ & 0 & 0 & 0 & 0 & 0 & 0 & 0 & 0 & 0 & 0 & 0 & 0 & 0 & $c_{6}$ & 0 \\
\hline$\{3,5\}$ & 0 & $c_{5}$ & 0 & $c_{4}$ & 0 & 0 & 0 & 0 & 0 & 0 & 0 & 0 & 0 & 0 & 0 & 0 & 0 & $c_{6}$ \\
\hline$\{4,6\}$ & 0 & 0 & $c_{5}$ & 0 & $c_{4}$ & 0 & 0 & 0 & 0 & $c_{6}$ & 0 & 0 & 0 & 0 & 0 & 0 & 0 & 0 \\
\hline$\{5,7\}$ & 0 & 0 & 0 & $c_{5}$ & 0 & $c_{4}$ & 0 & 0 & 0 & 0 & $c_{6}$ & 0 & 0 & 0 & 0 & 0 & 0 & 0 \\
\hline$\{6,8\}$ & 0 & 0 & 0 & 0 & $c_{5}$ & 0 & $c_{4}$ & 0 & 0 & 0 & 0 & $c_{6}$ & 0 & 0 & 0 & 0 & 0 & 0 \\
\hline$\{7,0\}$ & 0 & 0 & 0 & 0 & 0 & $c_{5}$ & 0 & $c_{4}$ & 0 & 0 & 0 & 0 & $c_{6}$ & 0 & 0 & 0 & 0 & 0 \\
\hline$\{8,1\}$ & 0 & 0 & 0 & 0 & 0 & 0 & $c_{5}$ & 0 & $c_{4}$ & 0 & 0 & 0 & 0 & $c_{6}$ & 0 & 0 & 0 & 0 \\
\hline$\{0,4\}$ & $c_{6}$ & 0 & 0 & 0 & 0 & 0 & 0 & 0 & 0 & $c_{4}$ & 0 & 0 & $c_{5}$ & 0 & 0 & 0 & 0 & 0 \\
\hline$\{1,5\}$ & 0 & $c_{6}$ & 0 & 0 & 0 & 0 & 0 & 0 & 0 & 0 & $c_{4}$ & 0 & 0 & $c_{5}$ & 0 & 0 & 0 & 0 \\
\hline$\{2,6\}$ & 0 & 0 & $c_{6}$ & 0 & 0 & 0 & 0 & 0 & 0 & 0 & 0 & $c_{4}$ & 0 & 0 & $c_{5}$ & 0 & 0 & 0 \\
\hline$\{3,7\}$ & 0 & 0 & 0 & $c_{6}$ & 0 & 0 & 0 & 0 & 0 & 0 & 0 & 0 & $c_{4}$ & 0 & 0 & $c_{5}$ & 0 & 0 \\
\hline$\{4,8\}$ & 0 & 0 & 0 & 0 & $c_{6}$ & 0 & 0 & 0 & 0 & 0 & 0 & 0 & 0 & $c_{4}$ & 0 & 0 & $c_{5}$ & 0 \\
\hline$\{5,0\}$ & 0 & 0 & 0 & 0 & 0 & $c_{6}$ & 0 & 0 & 0 & 0 & 0 & 0 & 0 & 0 & $c_{4}$ & 0 & 0 & $c_{5}$ \\
\hline$\{6,1\}$ & 0 & 0 & 0 & 0 & 0 & 0 & $c_{6}$ & 0 & 0 & $c_{5}$ & 0 & 0 & 0 & 0 & 0 & $c_{4}$ & 0 & 0 \\
\hline$\{7,2\}$ & 0 & 0 & 0 & 0 & 0 & 0 & 0 & $c_{6}$ & 0 & 0 & $c_{5}$ & 0 & 0 & 0 & 0 & 0 & $c_{4}$ & 0 \\
\hline$\{8,3\}$ & 0 & 0 & 0 & 0 & 0 & 0 & 0 & 0 & $c_{6}$ & 0 & 0 & $c_{5}$ & 0 & 0 & 0 & 0 & 0 & $c_{4}$ \\
\hline
\end{tabular}

Figure 3.1: Edge-Coloring Incidence Matrix of a $\operatorname{BIBD}(9,4,3)$. 
Lemma 3.2. There exists a properly colored $\operatorname{BIBD}(8,4, \lambda)$ for $\lambda=3 k, k \in \mathbb{Z}^{+}, k \geq 2$.

Proof: As in Lemma 2.4 we form an edge-coloring incidence matrix for a $\operatorname{BIBD}(8,4,3)$ as follows. Figure 3.2 represents the edge-coloring incidence matrix for the $\operatorname{BIBD}(8,4,3)$.

\begin{tabular}{|c|c|c|c|c|c|c|c|c|c|c|c|c|c|c|}
\hline & $B_{1}$ & $B_{2}$ & $B_{3}$ & $B_{4}$ & $B_{5}$ & $B_{6}$ & $B_{7}$ & $B_{8}$ & $B_{9}$ & $B_{10}$ & $B_{11}$ & $B_{12}$ & $B_{13}$ & $B_{14}$ \\
\hline$\{0,1\}$ & $c_{1}$ & 0 & 0 & 0 & 0 & 0 & 0 & $c_{2}$ & 0 & 0 & 0 & 0 & 0 & $c_{3}$ \\
\hline$\{1,2\}$ & 0 & $c_{1}$ & 0 & 0 & 0 & 0 & 0 & $c_{3}$ & $c_{2}$ & 0 & 0 & 0 & 0 & 0 \\
\hline$\{2,3\}$ & 0 & 0 & $c_{1}$ & 0 & 0 & 0 & 0 & 0 & $c_{3}$ & $c_{2}$ & 0 & 0 & 0 & 0 \\
\hline$\{3,4\}$ & 0 & 0 & 0 & $c_{1}$ & 0 & 0 & 0 & 0 & 0 & $c_{3}$ & $c_{2}$ & 0 & 0 & 0 \\
\hline$\{4,5\}$ & 0 & 0 & 0 & 0 & $c_{1}$ & 0 & 0 & 0 & 0 & 0 & $c_{3}$ & $c_{2}$ & 0 & 0 \\
\hline$\{5,6\}$ & 0 & 0 & 0 & 0 & 0 & $c_{1}$ & 0 & 0 & 0 & 0 & 0 & $c_{3}$ & $c_{2}$ & 0 \\
\hline$\{6,0\}$ & 0 & 0 & 0 & 0 & 0 & 0 & $c_{1}$ & 0 & 0 & 0 & 0 & 0 & $c_{3}$ & $c_{2}$ \\
\hline$\{0,2\}$ & 0 & 0 & 0 & 0 & 0 & 0 & $c_{2}$ & $c_{1}$ & 0 & 0 & 0 & 0 & $c_{4}$ & 0 \\
\hline$\{1,3\}$ & $c_{2}$ & 0 & 0 & 0 & 0 & 0 & 0 & 0 & $c_{1}$ & 0 & 0 & 0 & 0 & $c_{4}$ \\
\hline$\{2,4\}$ & 0 & $c_{2}$ & 0 & 0 & 0 & 0 & 0 & $c_{4}$ & 0 & $c_{1}$ & 0 & 0 & 0 & 0 \\
\hline$\{3,5\}$ & 0 & 0 & $c_{2}$ & 0 & 0 & 0 & 0 & 0 & $c_{4}$ & 0 & $c_{1}$ & 0 & 0 & 0 \\
\hline$\{4,6\}$ & 0 & 0 & 0 & $c_{2}$ & 0 & 0 & 0 & 0 & 0 & $c_{4}$ & 0 & $c_{1}$ & 0 & 0 \\
\hline$\{5,0\}$ & 0 & 0 & 0 & 0 & $c_{2}$ & 0 & 0 & 0 & 0 & 0 & $c_{4}$ & 0 & $c_{1}$ & 0 \\
\hline$\{6,1\}$ & 0 & 0 & 0 & 0 & 0 & $c_{2}$ & 0 & 0 & 0 & 0 & 0 & $c_{4}$ & 0 & $c_{1}$ \\
\hline$\{0,3\}$ & $c_{3}$ & 0 & 0 & 0 & 0 & 0 & 0 & 0 & 0 & 0 & $c_{6}$ & 0 & 0 & $c_{5}$ \\
\hline$\{1,4\}$ & 0 & $c_{3}$ & 0 & 0 & 0 & 0 & 0 & $c_{5}$ & 0 & 0 & 0 & $c_{6}$ & 0 & 0 \\
\hline$\{2,5\}$ & 0 & 0 & $c_{3}$ & 0 & 0 & 0 & 0 & 0 & $c_{5}$ & 0 & 0 & 0 & $c_{6}$ & 0 \\
\hline$\{3,6\}$ & 0 & 0 & 0 & $c_{3}$ & 0 & 0 & 0 & 0 & 0 & $c_{5}$ & 0 & 0 & 0 & $c_{6}$ \\
\hline$\{4,0\}$ & 0 & 0 & 0 & 0 & $c_{3}$ & 0 & 0 & $c_{6}$ & 0 & 0 & $c_{5}$ & 0 & 0 & 0 \\
\hline$\{5,1\}$ & 0 & 0 & 0 & 0 & 0 & $c_{3}$ & 0 & 0 & $c_{6}$ & 0 & 0 & $c_{5}$ & 0 & 0 \\
\hline$\{6,2\}$ & 0 & 0 & 0 & 0 & 0 & 0 & $c_{3}$ & 0 & 0 & $c_{6}$ & 0 & 0 & $c_{5}$ & 0 \\
\hline$\{0, \infty\}$ & $c_{4}$ & 0 & 0 & 0 & $c_{6}$ & 0 & $c_{5}$ & 0 & 0 & 0 & 0 & 0 & 0 & 0 \\
\hline$\{1, \infty\}$ & $c_{5}$ & $c_{4}$ & 0 & 0 & 0 & $c_{6}$ & 0 & 0 & 0 & 0 & 0 & 0 & 0 & 0 \\
\hline$\{2, \infty\}$ & 0 & $c_{5}$ & $c_{4}$ & 0 & 0 & 0 & $c_{6}$ & 0 & 0 & 0 & 0 & 0 & 0 & 0 \\
\hline$\{3, \infty\}$ & $c_{6}$ & 0 & $c_{5}$ & $c_{4}$ & 0 & 0 & 0 & 0 & 0 & 0 & 0 & 0 & 0 & 0 \\
\hline$\{4, \infty\}$ & 0 & $c_{6}$ & 0 & $c_{5}$ & $c_{4}$ & 0 & 0 & 0 & 0 & 0 & 0 & 0 & 0 & 0 \\
\hline$\{5, \infty\}$ & 0 & 0 & $c_{6}$ & 0 & $c_{5}$ & $c_{4}$ & 0 & 0 & 0 & 0 & 0 & 0 & 0 & 0 \\
\hline$\{6, \infty\}$ & 0 & 0 & 0 & $c_{6}$ & 0 & $c_{5}$ & $c_{4}$ & 0 & 0 & 0 & 0 & 0 & 0 & 0 \\
\hline
\end{tabular}

Figure 3.2: Edge-Coloring Incidence Matrix of a $\operatorname{BIBD}(8,4,3)$.

Let $A$ be the $8 \times 8$ circulant matrix whose first row is [01000000]. Then we can represent an edge-coloring incidence matrix for a $\operatorname{BIBD}(8,4,3)$ in terms of $A$ on the colors $\left\{c_{1}, c_{2}, c_{3}, c_{4}, c_{5}, c_{6}\right\}$. This representation is given in Figure 3.3 .

For any $i$, let $M_{i}^{(6)}$ be the edge-coloring incidence matrix of a $\operatorname{BIBD}(8,4,6)$ on the colors $c_{1+6 i}, c_{2+6 i}, c_{3+6 i}, c_{4+6 i}, c_{5+6 i}, c_{6+6 i}$ found in Figure 3.4. Denote $M_{0}^{(9)}$ as the edgecoloring incidence matrix of a $\operatorname{BIBD}(8,4,9)$ on the colors $c_{1^{\prime}}, c_{2^{\prime}}, \ldots, c_{9^{\prime}}$ found in Fig- 


\begin{tabular}{|c|c|}
\hline$c_{1} I$ & $c_{2} I+c_{3} A^{6}$ \\
\hline$c_{2} A^{6}$ & $c_{1} I+c_{4} A^{5}$ \\
\hline$c_{3} I$ & $c_{5} A^{3}+c_{6} A^{6}$ \\
\hline$c_{4} I+c_{6} A^{4}+c_{5} A^{6}$ & 0 \\
\hline
\end{tabular}

Figure 3.3: Edge-Coloring Incidence Matrix for a $\operatorname{BIBD}(8,4,3)$

$M_{i}^{(6)}=$\begin{tabular}{|c|c|c|c|}
\hline$\left(c_{1+6 i}\right) I$ & $\left(c_{2+6 i}\right) I+\left(c_{3+6 i}\right) A^{6}$ & $\left(c_{5+6 i}\right) I$ & $\left(c_{4+6 i}\right) I+\left(c_{6+6 i}\right) A^{6}$ \\
\hline$\left(c_{2+6 i}\right) A^{6}$ & $\left(c_{1+6 i}\right) I+\left(c_{4+6 i}\right) A^{5}$ & $\left(c_{6+6 i}\right) A^{6}$ & $\left(c_{3+6 i}\right) I+\left(c_{5+6 i}\right) A^{5}$ \\
\hline$\left(c_{3+6 i}\right) I$ & $\left(c_{5+6 i}\right) A^{3}+\left(c_{6+6 i}\right) A^{6}$ & $\left(c_{4+6 i}\right) I$ & $\left(c_{1+6 i}\right) A^{3}+\left(c_{2+6 i}\right) A^{6}$ \\
\hline $\begin{array}{c}\left(c_{4+6 i}\right) I \\
+\left(c_{6+6 i}\right) A^{4} \\
+\left(c_{5+6 i}\right) A^{6}\end{array}$ & 0 & $\begin{array}{c}\left(c_{1+6 i}\right) I \\
+\left(c_{2+6 i}\right) A^{4}\end{array}$ & 0 \\
\hline
\end{tabular}

Figure 3.4: Edge-Coloring Incidence Matrix for $\operatorname{BIBD}(8,4,6)$

ure 3.5. The subscripts are computed $(\bmod \lambda)$ where we identify $c_{0}$ with $c_{\lambda}$. In the case

\begin{tabular}{|c|c|c|c|c|c|c|}
\hline \multirow{4}{*}{$M_{0}^{(9)}=$} & $\left(c_{1^{\prime}}\right) I$ & $\left(c_{2^{\prime}}\right) I+\left(c_{3^{\prime}}\right) A^{6}$ & $\left(c_{5^{\prime}}\right) I$ & $\left(c_{4^{\prime}}\right) I+\left(c_{6^{\prime}}\right) A^{6}$ & $\left(c_{7^{\prime}}\right) I$ & $\left(c_{8^{\prime}}\right) I+\left(c_{9^{\prime}}\right) A^{6}$ \\
\hline & $\left(c_{2^{\prime}}\right) A^{6}$ & $\left(c_{1^{\prime}}\right) I+\left(c_{4^{\prime}}\right) A^{5}$ & $\left(c_{6^{\prime}}\right) A^{6}$ & $\left(c_{5^{\prime}}\right) I+\left(c_{8^{\prime}}\right) A^{5}$ & $\left(c_{9^{\prime}}\right) A^{6}$ & $\left(c_{3^{\prime}}\right) I+\left(c_{7^{\prime}}\right) A^{5}$ \\
\hline & $\left(c_{3^{\prime}}\right) I$ & $\left(c_{5^{\prime}}\right) A^{3}+\left(c_{6^{\prime}}\right) A^{6}$ & $\left(c_{4^{\prime}}\right) I$ & $\left(c_{7^{\prime}}\right) A^{3}+\left(c_{9^{\prime}}\right) A^{6}$ & $\left(c_{8^{\prime}}\right) I$ & $\left(c_{1^{\prime}}\right) A^{3}+\left(c_{2^{\prime}}\right) A^{6}$ \\
\hline & $\begin{array}{c}\left(c_{4^{\prime}}\right) I \\
+\left(c_{6^{\prime}}\right) A^{4} \\
+\left(c_{5^{\prime}}\right) A^{6}\end{array}$ & 0 & $\begin{aligned} & \left(c_{7^{\prime}}\right) I \\
+ & \left(c_{8^{\prime}}\right) A^{4} \\
+ & \left(c_{9^{\prime}}\right) A^{6}\end{aligned}$ & 0 & $\begin{aligned} & \left(c_{1^{\prime}}\right) I \\
+ & \left(c_{2^{\prime}}\right) A^{4} \\
+ & \left(c_{3^{\prime}}\right) A^{6}\end{aligned}$ & 0 \\
\hline
\end{tabular}

Figure 3.5: Edge-Coloring Incidence Matrix for a $\operatorname{BIBD}(8,4,9)$

where $\lambda=6 k$, our matrix is

$$
\begin{array}{l|l|l|l|}
\hline M_{0}^{(6)} & M_{1}^{(6)} & \cdots & M_{k-1}^{(6)} \\
\hline
\end{array}
$$

The set of colors used is $C_{0}, C_{1}, \ldots, C_{k-1}$ where

$$
C_{i}=\left\{c_{1+6 i}, c_{2+6 i}, c_{3+6 i}, c_{4+6 i}, c_{5+6 i}, c_{6+6 i}\right\}
$$

for $i=0,1, \ldots, k-1$. Because each $M_{i}^{(6)}$ is an edge coloring incidence matrix of a properly colored $\operatorname{BIBD}(8,4,6)$ on a different set of 6 colors, it follows that $M$ is an edgecoloring incidence matrix of a properly colored $\operatorname{BIBD}(8,4,6 k)$.

In the case where $\lambda=6 k+3$, our matrix is

$$
M=\begin{array}{|l|l|l|l|}
\hline M_{0}^{(9)} & M_{0}^{(6)} & \cdots & M_{k-2}^{(6)} \\
\hline
\end{array} .
$$

The set of colors used is $C, C_{0}, C_{1}, \ldots, C_{k-2}$ where $C=\left\{c_{1^{\prime}}, c_{2^{\prime}}, \ldots, c_{9^{\prime}}\right\}$ and

$$
C_{i}=\left\{c_{1+6 i}, c_{2+6 i}, c_{3+6 i}, c_{4+6 i}, c_{5+6 i}, c_{6+6 i}\right\}
$$


for $i=0,1, \ldots, k-2$. Because $M_{0}^{(9)}$ is an edge coloring incidence matrix of a properly colored $\operatorname{BIBD}(8,4,9)$ on 9 colors, and each $M_{i}^{(6)}$ is an edge coloring incidence matrix of a properly colored $\operatorname{BIBD}(8,4,6)$ on a different set of 6 colors which are all disjoint from the colors in $C$; it follows that $M$ is an edge-coloring incidence matrix of a properly colored $\operatorname{BIBD}(8,4,6 k+3)$.

Lemma 3.3. There exists a properly colored $\operatorname{BIBD}(12,4, \lambda)$ for $\lambda=3 k, k \geq 2, k \in \mathbb{Z}^{+}$.

Proof: As in Lemma 2.4 we form a colored edge-coloring incidence matrix for a $\operatorname{BIBD}(12,4,3)$ as follows. Let $A$ be the $12 \times 12$ circulant matrix whose first row is [010000000000]. Then the edge coloring incidence matrix of a $\operatorname{BIBD}(12,4,3)$ using 6 colors is given below.

\begin{tabular}{|c|c|c|c|}
\hline \multirow{6}{*}{$M_{i}=$} & $\left(c_{1+3 i}\right) I$ & $\left(c_{2+3 i}\right) A^{2}$ & $\left(c_{3+3 i}\right) A^{5}$ \\
\hline & $\left(c_{2+3 i}\right) A^{10}$ & $\left(c_{3+3 i}\right) A^{9}$ & $\left(c_{4+3 i}\right) A^{4}$ \\
\hline & $\left(c_{3+3 i}\right) I$ & $\left(c_{4+3 i}\right) A$ & $\left(c_{5+3 i}\right) A^{5}$ \\
\hline & $\left(c_{4+3 i}\right) A^{4}+\left(c_{5+3 i}\right) A^{8}$ & $\left(c_{6+3 i}\right) A^{2}$ & 0 \\
\hline & $\left(c_{6+3 i}\right) A^{4}$ & $\left(c_{1+3 i}\right) A+\left(c_{5+3 i}\right) A^{7}$ & 0 \\
\hline & 0 & 0 & $\left(c_{1+3 i}\right) A^{2}+\left(c_{2+3 i}\right) A^{4}+\left(c_{6+3 i}\right) A^{5}$ \\
\hline
\end{tabular}

Since $M_{i}$ is semi-properly colored, by Theorem 2.12 we can properly color a $\operatorname{BIBD}(12,4, \lambda)$.

Lemma 3.4. There exists a properly colored $\operatorname{BIBD}(7,4, \lambda)$ for $\lambda=2 k, k \in \mathbb{Z}^{+}, k \geq 3$.

Proof: As in Lemma 2.4 we form a colored edge-coloring incidence matrix for a $\operatorname{BIBD}(7,4,2)$ as follows. Figure 3.6 represents the edge-coloring incidence matrix for the $\operatorname{BIBD}(7,4,2)$.

Let $A$ be the $7 \times 7$ circulant matrix whose first row is [0100000]. Then the edge coloring incidence matrix of a $\operatorname{BIBD}(7,4,2)$ using 6 colors is given below.

$$
M_{i}=\begin{array}{|c|}
\hline\left(c_{1+2 i}\right) I+\left(c_{2+2 i}\right) A \\
\hline\left(c_{3+2 i}\right) I+\left(c_{4+2 i}\right) A^{2} \\
\hline\left(c_{5+2 i}\right) I+\left(c_{6+2 i}\right) A^{3} \\
\hline
\end{array}
$$

Since $M_{i}$ is semi-properly colored, by Theorem 2.12 we can properly color a $\operatorname{BIBD}(7,4, \lambda)$.

Lemma 3.5. There exists a properly colored $\operatorname{BIBD}(19,4, \lambda)$ for $\lambda=2 k, k \in \mathbb{Z}^{+}, k \geq 3$.

Proof: Let $A$ be the $19 \times 19$ circulant matrix whose first row is [0100000000000000000]. Then the edge coloring incidence matrix of a $\operatorname{BIBD}(19,4,2)$ using 6 colors is given in Figure 3.7. Since $M_{i}$ is semi-properly colored, by Theorem 2.12 we can properly color a $\operatorname{BIBD}(19,4, \lambda)$. 


\begin{tabular}{c|ccccccc|} 
& $B_{1}$ & $B_{2}$ & $B_{3}$ & $B_{4}$ & $B_{5}$ & $B_{6}$ & $B_{7}$ \\
\hline$\{0,1\}$ & $c_{1}$ & $c_{2}$ & 0 & 0 & 0 & 0 & 0 \\
$\{5,0\}$ & 0 & $c_{1}$ & $c_{2}$ & 0 & 0 & 0 & 0 \\
$\{3,4\}$ & 0 & 0 & $c_{1}$ & $c_{2}$ & 0 & 0 & 0 \\
$\{3,6\}$ & 0 & 0 & 0 & $c_{1}$ & $c_{2}$ & 0 & 0 \\
$\{2,5\}$ & 0 & 0 & 0 & 0 & $c_{1}$ & $c_{2}$ & 0 \\
$\{2,4\}$ & 0 & 0 & 0 & 0 & 0 & $c_{1}$ & $c_{2}$ \\
$\{0,2\}$ & $c_{2}$ & 0 & 0 & 0 & 0 & 0 & $c_{1}$ \\
\hline$\{0,3\}$ & $c_{3}$ & 0 & $c_{4}$ & 0 & 0 & 0 & 0 \\
$\{6,1\}$ & 0 & $c_{3}$ & 0 & $c_{4}$ & 0 & 0 & 0 \\
$\{3,5\}$ & 0 & 0 & $c_{3}$ & 0 & $c_{4}$ & 0 & 0 \\
$\{1,4\}$ & 0 & 0 & 0 & $c_{3}$ & 0 & $c_{4}$ & 0 \\
$\{6,2\}$ & 0 & 0 & 0 & 0 & $c_{3}$ & 0 & $c_{4}$ \\
$\{1,2\}$ & $c_{4}$ & 0 & 0 & 0 & 0 & $c_{3}$ & 0 \\
$\{6,0\}$ & 0 & $c_{4}$ & 0 & 0 & 0 & 0 & $c_{3}$ \\
\hline$\{1,3\}$ & $c_{5}$ & 0 & 0 & $c_{6}$ & 0 & 0 & 0 \\
$\{5,6\}$ & 0 & $c_{5}$ & 0 & 0 & $c_{6}$ & 0 & 0 \\
$\{4,5\}$ & 0 & 0 & $c_{5}$ & 0 & 0 & $c_{6}$ & 0 \\
$\{4,6\}$ & 0 & 0 & 0 & $c_{5}$ & 0 & 0 & $c_{6}$ \\
$\{2,3\}$ & $c_{6}$ & 0 & 0 & 0 & $c_{5}$ & 0 & 0 \\
$\{5,1\}$ & 0 & $c_{6}$ & 0 & 0 & 0 & $c_{5}$ & 0 \\
$\{4,0\}$ & 0 & 0 & $c_{6}$ & 0 & 0 & 0 & $c_{5}$ \\
\hline
\end{tabular}

Figure 3.6: Edge-Coloring Incidence Matrix of a $\operatorname{BIBD}(7,4,2)$.

$M_{i}=$\begin{tabular}{|c|c|c|}
\hline$\left(c_{1+2 i}\right) A^{16}$ & 0 & $\left(c_{2+2 i}\right) I$ \\
\hline$\left(c_{2+2 i}\right) A^{18}$ & 0 & $\left(c_{3+2 i}\right) A^{15}$ \\
\hline$\left(c_{3+2 i}\right) I$ & 0 & $\left(c_{4+2 i}\right) A^{13}$ \\
\hline 0 & $\left(c_{4+2 i}\right) A^{18}$ & $\left(c_{5+2 i}\right) I$ \\
\hline 0 & $\left(c_{5+2 i}\right) I$ & $\left(c_{6+2 i}\right) A^{15}$ \\
\hline 0 & $\left(c_{6+2 i}\right) A^{6}$ & $\left(c_{1+2 i}\right) I$ \\
\hline$\left(c_{4+2 i}\right) A^{7}$ & $\left(c_{1+2 i}\right) A^{5}$ & 0 \\
\hline$\left(c_{5+2 i}\right) A^{7}$ & $\left(c_{2+2 i}\right) A^{14}$ & 0 \\
\hline$\left(c_{6+2 i}\right) I$ & $\left(c_{3+2 i}\right) I$ & 0 \\
\hline
\end{tabular}

Figure 3.7: Edge-Coloring Incidence Matrix of a $\operatorname{BIBD}(19,4,2)$. 
Lemma 3.6. There exists a properly colored $\operatorname{BIBD}(10,4, \lambda)$ for $\lambda=2 k, k \in \mathbb{Z}^{+}, k \geq 4$.

Proof: We form an edge-coloring incidence matrix using 8 colors for a $\operatorname{BIBD}(10,4,2)$ as follows. Let $A_{i}^{(j)}$ be the following $5 \times 5$ matrices for $j=1,2,3$.

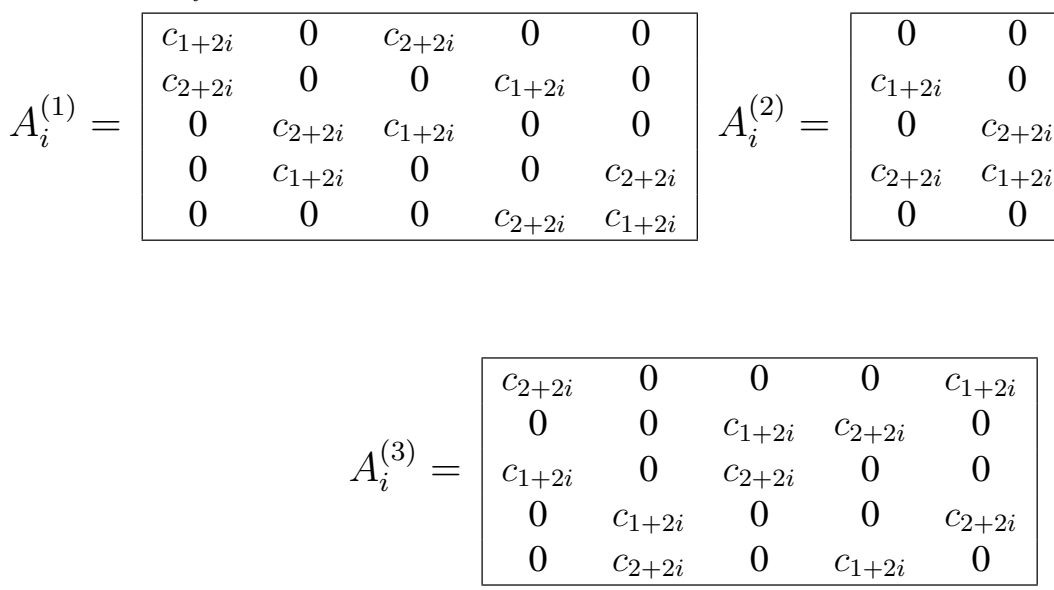

Let $B_{i}^{(j)}$ be the following $8 \times 5$ matrices for $j=1,2,3,4,5,6$.

\begin{tabular}{|c|c|c|c|c|c|c|c|c|c|c|c|}
\hline \multirow{8}{*}{$B_{i}^{(1)}=$} & $c_{3+2 i}$ & 0 & 0 & 0 & 0 & \multirow{8}{*}{$B_{i}^{(2)}=$} & 0 & $c_{4+2 i}$ & 0 & 0 & 0 \\
\hline & $c_{4+2 i}$ & 0 & 0 & 0 & 0 & & 0 & 0 & $c_{3+2 i}$ & 0 & 0 \\
\hline & 0 & 0 & 0 & $c_{3+2 i}$ & 0 & & $c_{4+2 i}$ & 0 & 0 & 0 & 0 \\
\hline & 0 & $c_{3+2 i}$ & 0 & 0 & 0 & & 0 & 0 & 0 & $c_{4+2 i}$ & 0 \\
\hline & 0 & $c_{4+2 i}$ & 0 & 0 & 0 & & 0 & 0 & 0 & 0 & $c_{3+2 i}$ \\
\hline & 0 & 0 & $c_{4+2 i}$ & 0 & 0 & & $c_{3+2 i}$ & 0 & 0 & 0 & 0 \\
\hline & 0 & 0 & 0 & $c_{4+2 i}$ & 0 & & 0 & $c_{3+2 i}$ & 0 & 0 & 0 \\
\hline & 0 & 0 & 0 & 0 & $c_{4+2 i}$ & & 0 & 0 & 0 & $c_{3+2 i}$ & 0 \\
\hline \multirow{8}{*}{$B_{i}^{(3)}=$} & $c_{5+2 i}$ & 0 & 0 & 0 & 0 & \multirow{8}{*}{$B_{i}^{(4)}=$} & $c_{6+2 i}$ & 0 & 0 & 0 & 0 \\
\hline & 0 & $c_{5+2 i}$ & 0 & 0 & 0 & & 0 & 0 & 0 & $c_{6+2 i}$ & 0 \\
\hline & 0 & 0 & $c_{5+2 i}$ & 0 & 0 & & 0 & $c_{6+2 i}$ & 0 & 0 & 0 \\
\hline & 0 & 0 & 0 & 0 & $c_{5+2 i}$ & & 0 & 0 & $c_{6+2 i}$ & 0 & 0 \\
\hline & 0 & 0 & $c_{6+2 i}$ & 0 & 0 & & 0 & 0 & $c_{5+2 i}$ & 0 & 0 \\
\hline & 0 & 0 & 0 & $c_{5+2 i}$ & 0 & & 0 & 0 & 0 & 0 & $c_{6+2 i}$ \\
\hline & 0 & 0 & 0 & 0 & $c_{6+2 i}$ & & $c_{5+2 i}$ & 0 & 0 & 0 & 0 \\
\hline & 0 & 0 & 0 & $c_{6+2 i}$ & 0 & & 0 & 0 & 0 & $c_{5+2 i}$ & 0 \\
\hline \multirow{8}{*}{$B_{i}^{(5)}=$} & 0 & 0 & 0 & 0 & $c_{7+2 i}$ & \multirow{8}{*}{$B_{i}^{(6)}=$} & 0 & 0 & $c_{8+2 i}$ & 0 & 0 \\
\hline & $c_{7+2 i}$ & 0 & 0 & 0 & 0 & & 0 & $c_{8+2 i}$ & 0 & 0 & 0 \\
\hline & 0 & 0 & $c_{8+2 i}$ & 0 & 0 & & 0 & 0 & 0 & 0 & $c_{7+2 i}$ \\
\hline & 0 & 0 & 0 & $c_{7+2 i}$ & 0 & & 0 & 0 & 0 & 0 & $c_{8+2 i}$ \\
\hline & 0 & 0 & 0 & 0 & $c_{8+2 i}$ & & 0 & 0 & 0 & $c_{7+2 i}$ & 0 \\
\hline & 0 & 0 & $c_{7+2 i}$ & 0 & 0 & & $c_{8+2 i}$ & 0 & 0 & 0 & 0 \\
\hline & 0 & $c_{8+2 i}$ & 0 & 0 & 0 & & 0 & 0 & $c_{7+2 i}$ & 0 & 0 \\
\hline & 0 & 0 & 0 & $c_{8+2 i}$ & 0 & & 0 & $c_{7+2 i}$ & 0 & 0 & 0 \\
\hline
\end{tabular}

Let $C_{i}^{(j)}$ be the following $5 \times 5$ matrices for $j=1,2,3$. 


$$
C_{i}^{(1)}=\begin{array}{ccccc}
c_{7+2 i} & c_{8+2 i} & 0 & 0 & 0 \\
0 & 0 & 0 & 0 & 0 \\
0 & 0 & 0 & 0 & 0 \\
0 & 0 & 0 & 0 & c_{7+2 i} \\
0 & 0 & c_{8+2 i} & 0 & 0 \\
0 & 0 & 0 & 0 & 0
\end{array} C_{i}^{(2)}=\begin{array}{ccccc|}
0 & 0 & 0 & 0 & 0 \\
0 & 0 & 0 & 0 & 0 \\
0 & 0 & c_{5+2 i} & 0 & c_{6+2 i} \\
c_{6+2 i} & 0 & 0 & 0 & 0 \\
0 & 0 & 0 & 0 & 0 \\
0 & c_{6+2 i} & 0 & 0 & 0 \\
\hline
\end{array}
$$

$$
C_{i}^{(3)}=\begin{array}{ccccc}
0 & 0 & 0 & 0 & 0 \\
c_{3+2 i} & 0 & 0 & c_{4+2 i} & 0 \\
0 & 0 & 0 & 0 & 0 \\
0 & 0 & 0 & 0 & 0 \\
0 & 0 & 0 & 0 & c_{3+2 i} \\
0 & c_{3+2 i} & 0 & 0 & 0 \\
\hline
\end{array}
$$

Now the edge-coloring incidence matrix can be represented by the above sub-matrices along with the all 0 sub-matrix.

$M_{i}=$\begin{tabular}{|c|c|c|}
\hline$A_{i}^{(1)}$ & 0 & 0 \\
\hline 0 & 0 & $A_{i}^{(2)}$ \\
\hline 0 & $A_{i}^{(3)}$ & 0 \\
\hline$B_{i}^{(1)}$ & $B_{i}^{(2)}$ & 0 \\
\hline$B_{i}^{(3)}$ & 0 & $B_{i}^{(4)}$ \\
\hline 0 & $B_{i}^{(5)}$ & $B_{i}^{(6)}$ \\
\hline$C_{i}^{(1)}$ & $C_{i}^{(2)}$ & $C_{i}^{(3)}$ \\
\hline
\end{tabular}

Since $M_{i}$ is semi-properly colored, by Theorem 2.12 we can properly color a $\operatorname{BIBD}(10,4, \lambda)$.

Because the process of checking each individual lemma for a correct direct construction is difficult, a program was built for the specific purpose of checking the small cases. Along with this is a csv (comma separated variables) and a tsv (tab separated variables) file of the edge-colored incidence matrix which are semi-properly colored. The program called bibdchecker.cpp is used to verify the design is a semi-properly colored BIBD. Mathematica was used to generate the properly colored versions of the BIBDs in csv and tsv form. Go to

http://www.mathlab.mtu.edu/ msjukuri/Data.html

for the program and corresponding csv and tsv files. 


\subsection{Main Results}

Before we can state the main theorem of this chapter, we must prove the general constructions we use. Note that the exponential notation in $4-\operatorname{GDD}\left(a_{1}^{b_{1}} a_{2}^{b_{2}} \cdots a_{x}^{b_{x}}\right)$ means we have $b_{1}$ groups of size $a_{1}, b_{2}$ groups of size $a_{2}, \ldots$, and $b_{x}$ groups of size $a_{x}$ in the GDD. We can use 4-GDDs and 4-RGDDs to build our $\operatorname{BIBD}(v, 4, \lambda) \mathrm{s}$ in a way that will allow us to properly color the edges. We now give some recursive constructions which are based off this idea.

Lemma 3.7. If there exists a $4-\operatorname{GDD}\left(a_{1}^{b_{1}} a_{2}^{b_{2}} \cdots a_{x}^{b_{x}}\right)$, and a properly colored $\operatorname{BIBD}\left(a_{i}, 4, \lambda\right)$ for all $i=1,2, \ldots, x$, then there exists a properly colored $\operatorname{BIBD}\left(\sum_{i=1}^{x} a_{i} b_{i}, 4, \lambda\right)$.

Proof: Repeat each of the blocks in a $4-\operatorname{GDD}\left(a_{1}^{b_{1}} a_{2}^{b_{2}} \cdots a_{x}^{b_{x}}\right) \lambda$ times. For each block, we must color each edge a different color using the colors $c_{i} \in\left\{c_{1}, \ldots, c_{\lambda}\right\}$. Each corresponding edge in the $\lambda$ copies of the blocks must also be a different color. So we color the edges in the $\lambda$ copies of each block as follows. Form a $6 \times \lambda$ matrix. The rows of the matrix will be indexed by the 6 edges of $K_{4}$, and the columns will be indexed by the $\lambda$ copies of the block. The entries of the matrix will be the first 6 rows of an $\operatorname{LS}(\lambda)$. Now the only pairs that have not been covered are the pairs which lie within the groups. So we place a properly colored $\operatorname{BIBD}\left(a_{i}, 4, \lambda\right)$ on each group for all $i=1,2, \ldots, x$. This forms a properly colored $\operatorname{BIBD}\left(\sum_{i=1}^{x} a_{i} b_{i}, 4, \lambda\right)$.

Lemma 3.8. If there exists a $4-\operatorname{GDD}\left(m^{u}\right)$ and a properly colored $\operatorname{BIBD}(m+1,4, \lambda)$, then there exists a properly colored $\operatorname{BIBD}(m u+1,4, \lambda)$.

Proof: Let $G_{i}$ for $i=1, \ldots, u$ be the $u$ groups of size $m$. Repeat each of the blocks in a $4-\operatorname{GDD}\left(m^{u}\right) \lambda$ times. For each block, we must color each edge a different color, using the colors $c_{i} \in\left\{c_{1}, \ldots, c_{\lambda}\right\}$. Each corresponding edge in the $\lambda$ copies of the block must also be a different color. So we color the edges in the $\lambda$ copies of each block as follows. Form a $6 \times \lambda$ matrix. The rows of the matrix will be indexed by the 6 edges of $K_{4}$, and the columns will be indexed by the $\lambda$ copies of the block. The entries of the matrix will be the first 6 rows of an $\operatorname{LS}(\lambda)$. Now the only pairs that have not been covered are the pairs which lie within the groups and pairs which contain the point $\{\infty\}$. So we place a properly colored $\mathrm{BIBD}(m+1,4, \lambda)$ on each $G_{i} \cup\{\infty\}$ for all $i=1,2, \ldots, u$. This forms a properly colored BIBD $(m u+1,4, \lambda)$.

Lemma 3.9. If there exists a $4-\operatorname{RGDD}\left(m^{u}\right)$, a properly colored $\operatorname{BIBD}(5,4, \lambda)$, a properly colored

$\operatorname{BIBD}(m, 4, \lambda)$, and a properly colored $\operatorname{BIBD}(t, 4, \lambda)$ for some $t \leq \frac{m(u-1)}{3}$, then there exists a properly colored $\operatorname{BIBD}(m u+t, 4, \lambda)$. 
Proof: Let $P_{i}$ for $i=1, \ldots, \frac{m(u-1)}{3}$ denote the parallel classes in the $4-\operatorname{RGDD}\left(m^{u}\right)$. Also let $\left\{\infty_{1}, \infty_{2}, \ldots\right.$,

$\left.\infty_{t}\right\}$ be $t$ new points where $0 \leq t \leq \frac{m(u-1)}{3}$. Consider the parallel class $P_{i}$ for $i=$ $1, \ldots, t$. We take each block of $P_{i}$ and join it with $\left\{\infty_{i}\right\}$. Now place a properly colored $\operatorname{BIBD}(5,4, \lambda)$ on each block of $P_{i} \cup\left\{\infty_{i}\right\}$. For each block in $P_{i}$ for $i=t+1, \ldots, \frac{m(u-1)}{3}$ we repeat it $\lambda$ times. We must color each edge of each block a different color, using the colors $c_{i} \in\left\{c_{1}, \ldots, c_{\lambda}\right\}$. Each corresponding edge in the $\lambda$ copies of the blocks must also be a different color. So we color the edges in the $\lambda$ copies of each block as follows. Form a $6 \times \lambda$ matrix. The rows of the matrix will be indexed by the 6 edges of $K_{4}$, and the columns will be indexed by the $\lambda$ copies of the block. The entries of the matrix will be the first 6 rows of a $\operatorname{LS}(\lambda)$. Now the only pairs that have not been covered are the pairs which lie within the groups and the pairs of the form $\left\{\left\{\infty_{i}, \infty_{j}\right\}: i, j \in\{1, \ldots, t\}\right\}$. So we place a properly colored $\operatorname{BIBD}(m, 4, \lambda)$ on each group, $G_{i}$, for all $i=1,2, \ldots, u$ and we place a properly colored $\operatorname{BIBD}(t, 4, \lambda)$ on the set of points $\left\{\infty_{1}, \ldots, \infty_{t}\right\}$. This forms a properly colored $\operatorname{BIBD}(m u+t, 4, \lambda)$.

The following theorem illustrates the use of the above lemmas.

Theorem 3.10. There exists a properly colored $\operatorname{BIBD}(v, 4, \lambda)$ for $v \equiv 0(\bmod 12)$ where $\lambda=3 k, k \geq 2$.

Proof: Let $v=24$. By Theorem 1.10 a $4-\operatorname{RGDD}\left(5^{4}\right)$ exists with 5 parallel classes, and Lemma 2.4 allows us to properly color a $\operatorname{BIBD}(5,4, \lambda)$ for $\lambda=3 k, k \in \mathbb{Z}^{+}, k \geq 2$. Therefore, we can apply Lemma 3.9 with $m=5, u=4$, and $t=4$ to obtain a properly colored $\operatorname{BIBD}(24,4, \lambda)$ for $\lambda=3 k k \geq 2$.

Let $v=36$. By Theorem 1.3 , there exists a $4-\operatorname{GDD}\left(9^{4}\right)$. From Lemma 3.1 we have a properly colored $\operatorname{BIBD}(9,4, \lambda)$ for $\lambda=3 k, k \geq 2$. Hence, we apply Lemma 3.7 with $x=1, a_{1}=9$, and $b_{1}=4$ to properly color a $\operatorname{BIBD}(36,4, \lambda)$ for $\lambda=3 k, k \geq 2$.

Now suppose $v=12 u$ where $u \geq 4$. There exists a $4-\operatorname{GDD}\left(12^{u}\right)$ for $u \geq 4$ by Theorem 1.3. By Lemma 3.3, we can properly color a $\operatorname{BIBD}(12,4, \lambda)$ design for $\lambda=3 k$, $k \geq 2$. Thus, we can let $x=1, a_{1}=12, b_{1}=u$, so it follows by Lemma 3.7 that we can properly color a $\operatorname{BIBD}(v, 4, \lambda)$ for $\lambda=3 k, k \geq 2$.

\subsection{1 $\lambda \equiv 0(\bmod 3)$}

In this section, we properly color all $\operatorname{BIBD}(v, 4, \lambda)$ s where $\lambda \equiv 0(\bmod 3)$. In this case, the necessary and sufficient conditions for the existence of $\operatorname{BIBD}(v, 4, \lambda)$ are $v \equiv 0,1$ $(\bmod 4)$. Note when $v \equiv 0,1(\bmod 4)$ and $\lambda \equiv 0(\bmod 6)$ these are already covered by Theorem 2.2, but the results in this section satisfy this case as well.

Theorem 3.11. There exists a proper coloring for every $\operatorname{BIBD}(v, 4, \lambda)$ for $\lambda=3 k, k \in$ $\mathbb{Z}^{+}, k \geq 2$, where $v \equiv 0,1(\bmod 4)$. 
Proof: Note that in each case we assume $\lambda=3 k, k \in \mathbb{Z}^{+}, k \geq 2$ unless otherwise stated. We will break this problem up into two main cases, $v \equiv 1(\bmod 4)$, and $v \equiv 0(\bmod 4)$.

Case 1: $\quad v \equiv 1(\bmod 4)$

Let $v \equiv 1(\bmod 4)$. Figure 3.8 represents the possible $v$ values.

\begin{tabular}{|c|c|c|}
\hline & 5 & 9 \\
\hline 13 & 17 & 21 \\
\hline 25 & 29 & 33 \\
\hline 37 & 41 & 45 \\
\hline 49 & 53 & 57 \\
\hline 61 & 65 & 69 \\
\hline$\vdots$ & $\vdots$ & $\vdots$ \\
\hline
\end{tabular}

Figure 3.8: Possible $v$ values for Case 1

Each column of Figure 3.8 represents $v \equiv 1(\bmod 4)$ in three different ways; $v \equiv 1$ $(\bmod 12), v \equiv 5(\bmod 12)$, and $v \equiv 9(\bmod 12)$.

Case 1.1: $v \equiv 1(\bmod 12)$

By Theorem 2.3, we can properly color $\operatorname{B} \operatorname{BBD}(v, 4, \lambda)$ where $v \equiv 1,4(\bmod 12)$.

Case 1.2: $\quad v \equiv 5(\bmod 12)$

For $v=5$, we can properly color a $\operatorname{BIBD}(5,4, \lambda)$ by Lemma 2.4

Let $v \equiv 5(\bmod 12)$. So $v=5+12 x=1+4(1+3 x)$ where $x \geq 1$. We construct a $4-\operatorname{GDD}\left(4^{u}\right)$ where $u=1+3 x$ and $x \geq 1$. This exists by Theorem 1.3 . We also know that a properly colored $\operatorname{BIBD}(5,4, \lambda)$ exists by Lemma 2.4 . So we apply Lemma 3.8 with $m=4, u=1+3 x, x \geq 1$.

Case 1.3: $v \equiv 9(\bmod 12)$

If $v \equiv 9(\bmod 12)$, then we have that either $v \equiv 9(\bmod 24)$ or $v \equiv 21(\bmod 24)$.

Case 1.3.1: $\quad v \equiv 9(\bmod 24)$

For $v=9$, we can properly color a $\operatorname{BIBD}(9,4, \lambda)$ by Lemma 3.1 .

For $v>9$, let $v=24 x+9=8(3 x+1)+1, x \geq 1$. Theorem 1.3 says that there exists a $4-\operatorname{GDD}\left(8^{u}\right)$ for $u=3 x+1$. Also by Lemma 3.1 there exists a properly colored $\operatorname{BIBD}(9,4, \lambda)$. So apply Lemma 3.8 with $m=8, u=3 x+1, x \geq 1$.

Case 1.3.2: $\quad v \equiv 21(\bmod 24)$ 
If $v \equiv 21(\bmod 24)$, then we can write $v$ as $v \equiv 21(\bmod 48)$ or $v \equiv 45(\bmod 48)$.

Case 1.3.2.1: $\quad v \equiv 45(\bmod 48)$

Suppose $v=48 x+45=4(12 x+11)+1$. We can construct a $4-\operatorname{GDD}\left(m^{4}\right)$ for $m=$ $12 x+11$ by Theorem 1.3 . By Theorem 3.10, we can properly color a $\operatorname{BIBD}(m+1,4, \lambda)$. So apply Lemma 3.8 to obtain a properly colored $\operatorname{BIBD}(4 m+1,4, \lambda)$.

Case 1.3.2.2: $\quad v \equiv 21(\bmod 48)$

If $v=21$, then we can write $v$ as $v=4(5)+1$. We can construct a $4-\operatorname{RGDD}\left(5^{4}\right)$ by Theorem 1.10. This has 5 parallel classes. Let $\{\infty\}$ be a new point, and let $P_{i}$ denote the $i^{\text {th }}$ parallel classes. We take each block of $P_{1}$ and join it with $\{\infty\}$. Place a properly colored $\operatorname{BIBD}(5,4, \lambda)$ design on each block of $P_{1} \cup\{\infty\}$. Now we repeat each block in $P_{i} \lambda$ times for $i=2,3,4,5$. We must color each edge of each block a different color, using the colors $c_{i} \in\left\{c_{1}, \ldots, c_{\lambda}\right\}$. Each corresponding edge in the $\lambda$ copies of the block must also be a different color. So we color the edges in the $\lambda$ copies of each block as follows. Form a $6 \times \lambda$ matrix. The rows of the matrix will be indexed by the 6 edges of $K_{4}$, and the columns will be indexed by the $\lambda$ copies of the block. The entries of the matrix will be the first 6 rows of a $\operatorname{LS}(\lambda)$. Now the only pairs that have not been covered are the pairs which lie within the groups. So we place a properly colored $\operatorname{BIBD}(5,4, \lambda)$ on each group $G_{i}$ for $i=1,2,3,4$. This forms a properly colored $\operatorname{BIBD}(21,4, \lambda)$.

Now suppose $v=21+48 x$ for $x \geq 1$. Since $v=21+48 x=4(12 x+4)+5$, we can construct a $4-\operatorname{RGDD}\left(m^{u}\right)$ with $m=12 x+5$ and $u=4$, by Theorem 1.10 . We also have that a properly colored $\operatorname{BIBD}(5,4, \lambda)$ design exists by Lemma 2.4, and a properly colored $\operatorname{BIBD}(12 x+4,4, \lambda)$ exists by Theorem 2.3 . Since $5 \leq 12 x+4$ for all $x>0$, we can apply Lemma 3.9.

Thus, we can properly color a $\operatorname{BIBD}(v, 4, \lambda)$ for $v \equiv 1(\bmod 4)$.

Case 2: $\quad v \equiv 0(\bmod 4)$

Let $v \equiv 0(\bmod 4)$. Figure 3.9 represents the possible $v$ values.

\begin{tabular}{|c|c|c|}
\hline & 8 & 12 \\
\hline 16 & 20 & 24 \\
\hline 28 & 32 & 36 \\
\hline 40 & 44 & 48 \\
\hline 52 & 56 & 60 \\
\hline 64 & 68 & 72 \\
\hline$\vdots$ & $\vdots$ & $\vdots$ \\
\hline
\end{tabular}

Figure 3.9: Possible $v$ values for Case 2

Each column of the above table represents $v \equiv 0(\bmod 4)$ in three different ways; 
$v \equiv 0(\bmod 12), v \equiv 4(\bmod 12)$, and $v \equiv 8(\bmod 12)$. Recall that if $v \equiv 0(\bmod 12)$, these designs were colored in Theorem 3.10 .

Case 2.1: $\quad v \equiv 4(\bmod 12)$

By Theorem 2.3, we can properly color a $\operatorname{BIBD}(v, 4, \lambda)$ for $v \equiv 4(\bmod 12)$.

Case 2.2: $\quad v \equiv 8(\bmod 12)$

If $v \equiv 8(\bmod 12)$, then we can rewrite $v$ as $v \equiv 8(\bmod 24)$ or $v \equiv 20(\bmod 24)$.

Case 2.2.1: $\quad v \equiv 8(\bmod 24)$

We can properly color a $\operatorname{BIBD}(8,4, \lambda)$ by Lemma 3.2. There exists a $4-\operatorname{GDD}\left(8^{u}\right)$ for $u=3 x+1$ and $x \geq 1$ by Theorem 1.3 . Therefore, we use Lemma 3.7 with $x=1, a_{1}=8$, and $b_{1}=u$.

Case 2.2.2: $\quad v \equiv 20(\bmod 24)$

We break this case into two subcases, $v \equiv 20(\bmod 48)$ and $v \equiv 44(\bmod 48)$.

Case 2.2.2.1: $\quad v \equiv 20(\bmod 48)$

Let $v=48 x+20=4(12 x+5)$ for $x \geq 0$. There exists a $4-\operatorname{GDD}\left(m^{4}\right)$ where $m=12 x+5$ by Theorem 1.3 . We properly color all $\operatorname{BIBD}(m, 4, \lambda) \mathrm{s}$ in Case 12 and Lemma 2.4. Thus we can apply Lemma 3.7 with $x=1, a_{1}=m$, and $b_{1}=4$.

Case 2.2.2.2: $\quad v \equiv 44(\bmod 48)$

Let $v=44+48 x=4(12 x+9)+8$ for $x \geq 0$. There exists a $4-\operatorname{RGDD}\left(m^{4}\right)$ for $m=12 x+9$ by Theorem 1.10 . This has $12 x+9$ parallel classes. We can properly color each $\operatorname{BIBD}(v, 4, \lambda)$ for $v \equiv 9(\bmod 12)$ by Case 13 , and we can properly color a $\operatorname{BIBD}(5,4, \lambda)$ using Lemma 2.4. Also, we can properly color a $\operatorname{BIBD}(8,4, \lambda)$ by Lemma 3.2. So we apply Lemma 3.9 with $m=12 x+9, u=4$, and $t=8$.

Therefore, we can properly color a $\operatorname{BIBD}(v, 4, \lambda)$ for $v \equiv 0(\bmod 4)$ and $\lambda=3 k$, $k \geq 2$. Furthermore, there exists a proper coloring for every $\operatorname{BIBD}(v, 4, \lambda)$ where $v \equiv 0,1$ $(\bmod 4)$.

\subsection{2 $\lambda \equiv 2,4(\bmod 6)$}

In this section, we properly color all $\operatorname{BIBD}(v, 4, \lambda) \mathrm{s}$ where $\lambda \equiv 2$ or $4(\bmod 6)$. In this case, the necessary and sufficient conditions for the existence of a $\operatorname{BIBD}(v, 4, \lambda)$ are that $v \equiv 1(\bmod 3)$. Note that when $v \equiv 1(\bmod 3)$ and $\lambda \equiv 0(\bmod 6)$, we could also use Theorem 2.2.

Theorem 3.12. There exists a proper coloring for every $\operatorname{BIBD}(v, 4, \lambda)$ design for $\lambda=$ $2 k, k \in \mathbb{Z}^{+}, k \geq 3$, where $v \equiv 1(\bmod 3)$. 
Proof: Note that in each case we assume $\lambda=2 k, k \in \mathbb{Z}^{+}, k \geq 3$ unless otherwise stated. Figure 3.10 represents the possible $v$ values when $v \equiv 1(\bmod 3)$.

\begin{tabular}{|c|c|c|c|}
\hline & 4 & 7 & 10 \\
\hline 13 & 16 & 19 & 22 \\
\hline 25 & 28 & 31 & 34 \\
\hline 37 & 40 & 43 & 46 \\
\hline 49 & 52 & 55 & 58 \\
\hline 61 & 64 & 67 & 70 \\
\hline$\vdots$ & $\vdots$ & $\vdots$ & $\vdots$ \\
\hline
\end{tabular}

Figure 3.10: Possible $v$ values for Theorem 3.12

Each column of the above table represents $v \equiv 1(\bmod 3)$ in four different ways; $v \equiv 1$ $(\bmod 12), v \equiv 4(\bmod 12), v \equiv 7(\bmod 12)$, and $v \equiv 10(\bmod 12)$.

Case 1: $v \equiv 1,4(\bmod 12)$

By Theorem 2.3, we can properly color $\operatorname{B} \operatorname{IBD}(v, 4, \lambda)$ for $v \equiv 1,4(\bmod 12)$.

Case 2: $\quad v \equiv 7(\bmod 12)$

We can properly color a $\operatorname{BIBD}(7,4, \lambda)$ for $\lambda=2 k, k \geq 3$ by Lemma 3.4. We can also properly color a $\operatorname{BIBD}(19,4, \lambda)$ for all such $\lambda$ by Lemma 3.5 . Let $v=12 x+7=$ $6(2 x+1)+1$ for $x \geq 2$. By Theorem 1.3 , there exists a $4-\operatorname{GDD}\left(6^{2 x+1}\right)$ for all $x \geq 2$. So we can apply Lemma 3.8 with $m=6$ and $u=2 x+1$.

Case 3: $v \equiv 10(\bmod 12)$

If $v=10$, we can properly color a $\operatorname{BIBD}(10,4, \lambda)$ with $\lambda=6$ by Theorem 2.2 . Then for all $\lambda=2 k, k \geq 4$, we apply Lemma 3.6.

If $v=22$, we can apply Lemma 3.7 with $x=2, a_{1}=1, b_{1}=15, a_{2}=7$, and $b_{2}=1$. Note that the required $4-\operatorname{GDD}\left(1^{15} 7^{1}\right)$ exists by Theorem 1.6 , and a properly colored $\operatorname{BIBD}(7,4, \lambda)$ exists by Lemma 3.4 .

Now let $v=12 x+10$, with $x \geq 2$. There exists a $4-\operatorname{GDD}\left(4^{u} m^{1}\right)$ where $m=10$ and $u=3 x$ for all $x \geq 2$ by Theorem 1.4 . So we let $x=2, a_{1}=4, b_{1}=u, a_{2}=m$, and $b_{2}=1$; and we apply Lemma 3.7. The required properly colored $\operatorname{BIBD}(10,4, \lambda)$ as stated above.

\subsection{Conclusion}

We are now in a position to prove the main theorem.

Theorem 3.13. There is a proper edge coloring for every $\operatorname{BIBD}(v, 4, \lambda)$ where $\lambda \geq 6$. 
Proof: Recall the necessary and sufficient conditions for the existence of a $\operatorname{BIBD}(v, 4, \lambda)$.

If $\lambda \equiv 1,5(\bmod 6)$, then $v \equiv 1,4(\bmod 12)$;

If $\lambda \equiv 2,4(\bmod 6)$, then $v \equiv 1(\bmod 3)$;

If $\lambda \equiv 3(\bmod 6)$, then $v \equiv 0,1(\bmod 4)$; and

If $\lambda \equiv 0(\bmod 6)$, then $v \geq 4$.

If $\lambda \equiv 1,5(\bmod 6)$, then $v \equiv 1,4(\bmod 12)$ and we apply Theorem 2.3 to properly color a $\operatorname{BIBD}(v, 4, \lambda)$. If $\lambda \equiv 0(\bmod 6)$, then $v \geq 4$ and we can properly color a $\operatorname{BIBD}(v, 4, \lambda)$ by applying Theorem 2.2. If $\lambda \equiv 3(\bmod 6)$, then $v \equiv 0,1(\bmod 4)$ and we can apply Theorem 3.11. Finally, if $\lambda \equiv 2,4(\bmod 6)$, then $v \equiv 1(\bmod 3)$ and we apply Theorem 3.12 . 


\section{Chapter 4}

\section{Proper Edge Coloring With Block Size 5}

Again, we first need to understand the construction in Chapter 2 before adventuring into this chapter. Our goal in this chapter is to prove we can properly color all $\operatorname{BIBD}(v, 5, \lambda)$ except possibly for a few cases. To prove this we must first show there exists properly colored $\operatorname{BIBD}(v, 5, \lambda)$ for small $v$. Afterwords, we prove the generalized constructions hold which are then used on the main theorem.

\subsection{Direct Constructions With Block Size 5}

Lemma 4.1. There exists a properly colored $\operatorname{BIBD}(11,5, \lambda)$ for $\lambda=2 k, k \in \mathbb{Z}^{+}, k \geq 5$.

Proof: We form an edge-coloring incidence matrix using 10 colors for a $\operatorname{BIBD}(11,5,2)$. Figure 4.1 represents the edge-coloring incidence matrix for the $\operatorname{BIBD}(11,5,2)$.

Let $A$ be the $11 \times 11$ circulant matrix whose first row is [01000000000]. Then the edge-coloring incidence matrix of a $\operatorname{BIBD}(11,5,2)$ using 10 colors is given below.

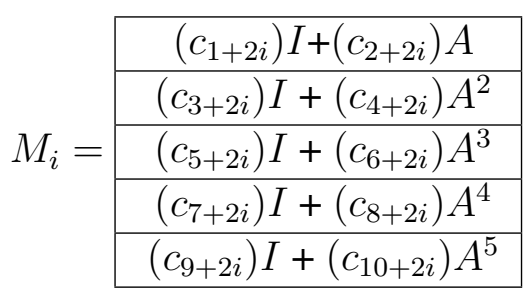

Since $M_{i}$ is semi-properly colored, by applying Theorem 2.12 we can properly color a $\operatorname{BIBD}(11,5, \lambda)$.

Lemma 4.2. There exists a properly colored $\operatorname{BIBD}(31,5, \lambda)$ for $\lambda=2 k, k \in \mathbb{Z}^{+}, k \geq 5$.

Proof: Let $A$ be the $31 \times 31$ circulant matrix whose first row is

$$
\text { [0100000000000000000000000000000]. }
$$

Then the edge coloring incidence matrix of a $\operatorname{BIBD}(31,5,2)$ using 10 colors in Figure 4.2 


\begin{tabular}{|c|c|c|c|c|c|c|c|c|c|c|c|}
\hline & $B_{1}$ & $B_{2}$ & $B_{3}$ & $B_{4}$ & $B_{5}$ & $B_{6}$ & $B_{7}$ & $B_{8}$ & $B_{9}$ & $B_{10}$ & $B_{11}$ \\
\hline$\{0,1\}$ & $c_{1}$ & $c_{2}$ & 0 & 0 & 0 & 0 & 0 & 0 & 0 & 0 & 0 \\
\hline$\{0,5\}$ & 0 & $c_{1}$ & $c_{2}$ & 0 & 0 & 0 & 0 & 0 & 0 & 0 & 0 \\
\hline$\{8,0\}$ & 0 & 0 & $c_{1}$ & $c_{2}$ & 0 & 0 & 0 & 0 & 0 & 0 & 0 \\
\hline$\{10,0\}$ & 0 & 0 & 0 & $c_{1}$ & $c_{2}$ & 0 & 0 & 0 & 0 & 0 & 0 \\
\hline$\{10,4\}$ & 0 & 0 & 0 & 0 & $c_{1}$ & $c_{2}$ & 0 & 0 & 0 & 0 & 0 \\
\hline$\{10,1\}$ & 0 & 0 & 0 & 0 & 0 & $c_{1}$ & $c_{2}$ & 0 & 0 & 0 & 0 \\
\hline$\{9,1\}$ & 0 & 0 & 0 & 0 & 0 & 0 & $c_{1}$ & $c_{2}$ & 0 & 0 & 0 \\
\hline$\{6,9\}$ & 0 & 0 & 0 & 0 & 0 & 0 & 0 & $c_{1}$ & $c_{2}$ & 0 & 0 \\
\hline$\{2,6\}$ & 0 & 0 & 0 & 0 & 0 & 0 & 0 & 0 & $c_{1}$ & $c_{2}$ & 0 \\
\hline$\{5,7\}$ & 0 & 0 & 0 & 0 & 0 & 0 & 0 & 0 & 0 & $c_{1}$ & $c_{2}$ \\
\hline$\{3,7\}$ & $c_{2}$ & 0 & 0 & 0 & 0 & 0 & 0 & 0 & 0 & 0 & $c_{1}$ \\
\hline$\{0,2\}$ & $c_{3}$ & 0 & $c_{4}$ & 0 & 0 & 0 & 0 & 0 & 0 & 0 & 0 \\
\hline$\{6,0\}$ & 0 & $c_{3}$ & 0 & $c_{4}$ & 0 & 0 & 0 & 0 & 0 & 0 & 0 \\
\hline$\{9,0\}$ & 0 & 0 & $c_{3}$ & 0 & $c_{4}$ & 0 & 0 & 0 & 0 & 0 & 0 \\
\hline$\{8,10\}$ & 0 & 0 & 0 & $c_{3}$ & 0 & $c_{4}$ & 0 & 0 & 0 & 0 & 0 \\
\hline$\{9,10\}$ & 0 & 0 & 0 & 0 & $c_{3}$ & 0 & $c_{4}$ & 0 & 0 & 0 & 0 \\
\hline$\{8,1\}$ & 0 & 0 & 0 & 0 & 0 & $c_{3}$ & 0 & $c_{4}$ & 0 & 0 & 0 \\
\hline$\{9,3\}$ & 0 & 0 & 0 & 0 & 0 & 0 & $c_{3}$ & 0 & $c_{4}$ & 0 & 0 \\
\hline$\{6,7\}$ & 0 & 0 & 0 & 0 & 0 & 0 & 0 & $c_{3}$ & 0 & $c_{4}$ & 0 \\
\hline$\{3,4\}$ & 0 & 0 & 0 & 0 & 0 & 0 & 0 & 0 & $c_{3}$ & 0 & $c_{4}$ \\
\hline$\{2,7\}$ & $c_{4}$ & 0 & 0 & 0 & 0 & 0 & 0 & 0 & 0 & $c_{3}$ & 0 \\
\hline$\{4,5\}$ & 0 & $c_{4}$ & 0 & 0 & 0 & 0 & 0 & 0 & 0 & 0 & $c_{3}$ \\
\hline$\{0,3\}$ & $c_{5}$ & 0 & 0 & $c_{6}$ & 0 & 0 & 0 & 0 & 0 & 0 & 0 \\
\hline$\{0,4\}$ & 0 & $c_{5}$ & 0 & 0 & $c_{6}$ & 0 & 0 & 0 & 0 & 0 & 0 \\
\hline$\{8,2\}$ & 0 & 0 & $c_{5}$ & 0 & 0 & $c_{6}$ & 0 & 0 & 0 & 0 & 0 \\
\hline$\{10,3\}$ & 0 & 0 & 0 & $c_{5}$ & 0 & 0 & $c_{6}$ & 0 & 0 & 0 & 0 \\
\hline$\{7,9\}$ & 0 & 0 & 0 & 0 & $c_{5}$ & 0 & 0 & $c_{6}$ & 0 & 0 & 0 \\
\hline$\{2,4\}$ & 0 & 0 & 0 & 0 & 0 & $c_{5}$ & 0 & 0 & $c_{6}$ & 0 & 0 \\
\hline$\{5,10\}$ & 0 & 0 & 0 & 0 & 0 & 0 & $c_{5}$ & 0 & 0 & $c_{6}$ & 0 \\
\hline$\{7,8\}$ & 0 & 0 & 0 & 0 & 0 & 0 & 0 & $c_{5}$ & 0 & 0 & $c_{6}$ \\
\hline$\{2,3\}$ & $c_{6}$ & 0 & 0 & 0 & 0 & 0 & 0 & 0 & $c_{5}$ & 0 & 0 \\
\hline$\{5,6\}$ & 0 & $c_{6}$ & 0 & 0 & 0 & 0 & 0 & 0 & 0 & $c_{5}$ & 0 \\
\hline$\{5,8\}$ & 0 & 0 & $c_{6}$ & 0 & 0 & 0 & 0 & 0 & 0 & 0 & $c_{5}$ \\
\hline$\{7,0\}$ & $c_{7}$ & 0 & 0 & 0 & $c_{8}$ & 0 & 0 & 0 & 0 & 0 & 0 \\
\hline$\{1,4\}$ & 0 & $c_{7}$ & 0 & 0 & 0 & $c_{8}$ & 0 & 0 & 0 & 0 & 0 \\
\hline$\{5,9\}$ & 0 & 0 & $c_{7}$ & 0 & 0 & 0 & $c_{8}$ & 0 & 0 & 0 & 0 \\
\hline$\{6,8\}$ & 0 & 0 & 0 & $c_{7}$ & 0 & 0 & 0 & $c_{8}$ & 0 & 0 & 0 \\
\hline$\{4,9\}$ & 0 & 0 & 0 & 0 & $c_{7}$ & 0 & 0 & 0 & $c_{8}$ & 0 & 0 \\
\hline$\{10,2\}$ & 0 & 0 & 0 & 0 & 0 & $c_{7}$ & 0 & 0 & 0 & $c_{8}$ & 0 \\
\hline$\{3,5\}$ & 0 & 0 & 0 & 0 & 0 & 0 & $c_{7}$ & 0 & 0 & 0 & $c_{8}$ \\
\hline$\{7,1\}$ & $c_{8}$ & 0 & 0 & 0 & 0 & 0 & 0 & $c_{7}$ & 0 & 0 & 0 \\
\hline$\{4,6\}$ & 0 & $c_{8}$ & 0 & 0 & 0 & 0 & 0 & 0 & $c_{7}$ & 0 & 0 \\
\hline$\{2,5\}$ & 0 & 0 & $c_{8}$ & 0 & 0 & 0 & 0 & 0 & 0 & $c_{7}$ & 0 \\
\hline$\{3,8\}$ & 0 & 0 & 0 & $c_{8}$ & 0 & 0 & 0 & 0 & 0 & 0 & $c_{7}$ \\
\hline$\{1,2\}$ & $c_{9}$ & 0 & 0 & 0 & 0 & $c_{10}$ & 0 & 0 & 0 & 0 & 0 \\
\hline$\{1,5\}$ & 0 & $c_{9}$ & 0 & 0 & 0 & 0 & $c_{10}$ & 0 & 0 & 0 & 0 \\
\hline$\{8,9\}$ & 0 & 0 & $c_{9}$ & 0 & 0 & 0 & 0 & $c_{10}$ & 0 & 0 & 0 \\
\hline$\{3,6\}$ & 0 & 0 & 0 & $c_{9}$ & 0 & 0 & 0 & 0 & $c_{10}$ & 0 & 0 \\
\hline$\{7,10\}$ & 0 & 0 & 0 & 0 & $c_{9}$ & 0 & 0 & 0 & 0 & $c_{10}$ & 0 \\
\hline$\{4,8\}$ & 0 & 0 & 0 & 0 & 0 & $c_{9}$ & 0 & 0 & 0 & 0 & $c_{10}$ \\
\hline$\{1,3\}$ & $c_{10}$ & 0 & 0 & 0 & 0 & 0 & $c_{9}$ & 0 & 0 & 0 & 0 \\
\hline$\{1,6\}$ & 0 & $c_{10}$ & 0 & 0 & 0 & 0 & 0 & $c_{9}$ & 0 & 0 & 0 \\
\hline$\{9,2\}$ & 0 & 0 & $c_{10}$ & 0 & 0 & 0 & 0 & 0 & $c_{9}$ & 0 & 0 \\
\hline$\{6,10\}$ & 0 & 0 & 0 & $c_{10}$ & 0 & 0 & 0 & 0 & 0 & $c_{9}$ & 0 \\
\hline$\{4,7\}$ & 0 & 0 & 0 & 0 & $c_{10}$ & 0 & 0 & 0 & 0 & 0 & $c_{9}$ \\
\hline
\end{tabular}

Figure 4.1: Edge-Coloring Incidence Matrix of a $\operatorname{BIBD}(11,5,2)$. 


$M_{i}=$\begin{tabular}{|c|c|c|}
\hline$\left(c_{1+2 i}\right) I$ & 0 & $\left(c_{2+2 i}\right) A^{27}$ \\
\hline$\left(c_{2+2 i}\right) A^{30}$ & 0 & $\left(c_{3+2 i}\right) A^{18}$ \\
\hline$\left(c_{3+2 i}\right) I$ & $\left(c_{2+2 i}\right) I$ & 0 \\
\hline$\left(c_{4+2 i}\right) A^{28}$ & 0 & $\left(c_{1+2 i}\right) I$ \\
\hline 0 & $\left(c_{1+2 i}\right) A^{22}$ & $\left(c_{4+2 i}\right) I$ \\
\hline$\left(c_{5+2 i}\right) A^{30}$ & $\left(c_{4+2 i}\right) A^{28}$ & 0 \\
\hline$\left(c_{6+2 i}\right) I$ & $\left(c_{3+2 i}\right) A^{17}$ & 0 \\
\hline$\left(c_{7+2 i}\right) A^{24}$ & 0 & $\left(c_{6+2 i}\right) A^{26}$ \\
\hline 0 & $\left(c_{6+2 i}\right) I$ & $\left(c_{5+2 i}\right) A^{27}$ \\
\hline 0 & $\left(c_{5+2 i}\right) A^{10}$ & $\left(c_{8+2 i}\right) A^{26}$ \\
\hline 0 & $\left(c_{8+2 i}\right) A^{28}$ & $\left(c_{7+2 i}\right) A^{27}$ \\
\hline$\left(c_{8+2 i}\right) A^{28}$ & $\left(c_{9+2 i}\right) A^{22}$ & 0 \\
\hline 0 & $\left(c_{10+2 i}\right) A^{10}$ & $\left(c_{9+2 i}\right) I$ \\
\hline$\left(c_{10+2 i}\right) A^{30}$ & $\left(c_{7+2 i}\right) I$ & 0 \\
\hline$\left(c_{9+2 i}\right) I$ & 0 & $\left(c_{10+2 i}\right) I$ \\
\hline
\end{tabular}

Figure 4.2: Edge-Coloring Incidence Matrix of a $\operatorname{BIBD}(31,5,2)$.

Since $M_{i}$ is semi-properly colored, by applying Theorem 2.12 we can properly color a $\operatorname{BIBD}(31,5, \lambda)$.

Lemma 4.3. There exists a properly colored $\operatorname{BIBD}(51,5, \lambda)$ for $\lambda=2 k, k \in \mathbb{Z}^{+}, k \geq 5$.

Proof: Let $A$ be the $51 \times 51$ circulant matrix whose first row is

$$
\text { [010000000000000000000000000000000000000000000000000]. }
$$

Then the edge coloring incidence matrix of a $\operatorname{BIBD}(51,5,2)$ using 10 colors in Figure 4.3

Since $M_{i}$ is semi-properly colored, by applying Theorem 2.12 we can properly color a $\operatorname{BIBD}(51,5, \lambda)$.

Lemma 4.4. There exists a properly colored $\operatorname{BIBD}(6,5, \lambda)$ for $\lambda=4 k, k \in \mathbb{Z}^{+}, k \geq 3$. Proof: The incidence matrix of a $\operatorname{BIBD}(6,5,4)$ is

\begin{tabular}{c|cccccc|} 
& $B_{1}$ & $B_{2}$ & $B_{3}$ & $B_{4}$ & $B_{5}$ & $B_{6}$ \\
\hline 0 & 0 & 1 & 1 & 1 & 1 & 1 \\
1 & 1 & 0 & 1 & 1 & 1 & 1 \\
2 & 1 & 1 & 0 & 1 & 1 & 1 \\
3 & 1 & 1 & 1 & 0 & 1 & 1 \\
4 & 1 & 1 & 1 & 1 & 0 & 1 \\
5 & 1 & 1 & 1 & 1 & 1 & 0 \\
\hline
\end{tabular}

Figure 4.4 represents the edge-coloring incidence matrix for the $\operatorname{BIBD}(6,5,4)$. 


\begin{tabular}{|c|c|c|c|c|c|}
\hline & $\left(c_{1+4 i}\right) I$ & $\left(c_{2+4 i}\right) I$ & 0 & 0 & 0 \\
\hline & 0 & 0 & $\left(c_{1+4 i}\right) A^{35}$ & 0 & $\left(c_{2+4 i}\right) A^{41}$ \\
\hline & 0 & 0 & 0 & $\left(c_{1+4 i}\right) A^{15}$ & $\left(c_{4+4 i}\right) A^{39}$ \\
\hline & $\left(c_{2+4 i}\right) A^{20}$ & 0 & 0 & 0 & $\left(c_{3+4 i}\right) I$ \\
\hline & 0 & 0 & $\left(c_{2+4 i}\right) A^{40}$ & 0 & $\left(c_{1+4 i}\right) A^{41}$ \\
\hline & 0 & 0 & 0 & $\left(c_{2+4 i}\right) A^{44}$ & $\left(c_{5+4 i}\right) A^{47}$ \\
\hline & 0 & 0 & $\left(c_{3+4 i}\right) A^{40}$ & $\left(c_{4+4 i}\right) I$ & 0 \\
\hline & 0 & $\left(c_{1+4 i}\right) A^{50}$ & 0 & 0 & $\left(c_{6+4 i}\right) A^{47}$ \\
\hline & 0 & $\left(c_{3+4 i}\right) I$ & $\left(c_{4+4 i}\right) A^{9}$ & 0 & 0 \\
\hline & 0 & $\left(c_{4+4 i}\right) A^{28}$ & 0 & 0 & $\left(c_{7+4 i}\right) I$ \\
\hline & 0 & 0 & $\left(c_{5+4 i}\right) I$ & 0 & $\left(c_{8+4 i}\right) A^{47}$ \\
\hline & 0 & 0 & 0 & $\left(c_{6+4 i}\right) A^{12}$ & $\left(c_{9+4 i}\right) I$ \\
\hline$M_{i}=$ & $\left(c_{4+4 i}\right) A^{50}$ & 0 & 0 & $\left(c_{3+4 i}\right) I$ & 0 \\
\hline & $\left(c_{3+4 i}\right) I$ & $\left(c_{6+4 i}\right) A^{42}$ & 0 & 0 & 0 \\
\hline & 0 & 0 & 0 & $\left(c_{7+4 i}\right) A^{15}$ & $\left(c_{10+4 i}\right) I$ \\
\hline & $\left(c_{5+4 i}\right) A^{16}$ & 0 & $\left(c_{6+4 i}\right) I$ & 0 & 0 \\
\hline & $\left(c_{6+4 i}\right) A^{16}+\left(c_{7+4 i}\right) A^{37}$ & 0 & 0 & 0 & 0 \\
\hline & 0 & $\left(c_{5+4 i}\right) A^{18}$ & $\left(c_{8+4 i}\right) I$ & 0 & 0 \\
\hline & 0 & $\left(c_{7+4 i}\right) A^{18}$ & 0 & $\left(c_{8+4 i}\right) A^{12}$ & 0 \\
\hline & $\left(c_{8+4 i}\right) A^{20}$ & 0 & $\left(c_{7+4 i}\right) A^{9}$ & 0 & 0 \\
\hline & $\left(c_{9+4 i}\right) A^{20}+\left(c_{10+4 i}\right) A^{37}$ & 0 & 0 & 0 & 0 \\
\hline & 0 & $\left(c_{8+4 i}\right) A^{50}$ & 0 & $\left(c_{5+4 i}\right) A^{15}$ & 0 \\
\hline & 0 & $\left(c_{9+4 i}\right) I$ & 0 & $\left(c_{10+4 i}\right) A^{38}$ & 0 \\
\hline & 0 & $\left(c_{10+4 i}\right) A^{42}$ & $\left(c_{9+4 i}\right) A^{33}$ & 0 & 0 \\
\hline & 0 & 0 & $\left(c_{10+4 i}\right) A^{9}$ & $\left(c_{9+4 i}\right) A^{12}$ & 0 \\
\hline
\end{tabular}

Figure 4.3: Edge-Coloring Incidence Matrix of a $\operatorname{BIBD}(51,5,2)$. 


\begin{tabular}{c|cccccc|} 
& $B_{1}$ & $B_{2}$ & $B_{3}$ & $B_{4}$ & $B_{5}$ & $B_{6}$ \\
\hline$\{0,1\}$ & 0 & 0 & $c_{1}$ & $c_{2}$ & $c_{3}$ & $c_{4}$ \\
$\{1,2\}$ & $c_{4}$ & 0 & 0 & $c_{1}$ & $c_{2}$ & $c_{3}$ \\
$\{2,3\}$ & $c_{3}$ & $c_{4}$ & 0 & 0 & $c_{1}$ & $c_{2}$ \\
$\{3,4\}$ & $c_{2}$ & $c_{3}$ & $c_{4}$ & 0 & 0 & $c_{1}$ \\
$\{4,5\}$ & $c_{1}$ & $c_{2}$ & $c_{3}$ & $c_{4}$ & 0 & 0 \\
$\{5,0\}$ & 0 & $c_{1}$ & $c_{2}$ & $c_{3}$ & $c_{4}$ & 0 \\
\hline$\{0,2\}$ & 0 & $c_{5}$ & 0 & $c_{6}$ & $c_{7}$ & $c_{8}$ \\
$\{1,3\}$ & $c_{8}$ & 0 & $c_{5}$ & 0 & $c_{6}$ & $c_{7}$ \\
$\{2,4\}$ & $c_{7}$ & $c_{8}$ & 0 & $c_{5}$ & 0 & $c_{6}$ \\
$\{3,5\}$ & $c_{6}$ & $c_{7}$ & $c_{8}$ & 0 & $c_{5}$ & 0 \\
$\{4,0\}$ & 0 & $c_{6}$ & $c_{7}$ & $c_{8}$ & 0 & $c_{5}$ \\
$\{5,1\}$ & $c_{5}$ & 0 & $c_{6}$ & $c_{7}$ & $c_{8}$ & 0 \\
\hline$\{0,3\}$ & 0 & $c_{9}$ & $c_{10}$ & 0 & $c_{11}$ & $c_{12}$ \\
$\{1,4\}$ & $c_{12}$ & 0 & $c_{9}$ & $c_{10}$ & 0 & $c_{11}$ \\
$\{2,5\}$ & $c_{11}$ & $c_{12}$ & 0 & $c_{9}$ & $c_{10}$ & 0 \\
\hline
\end{tabular}

Figure 4.4: Edge-Coloring Incidence Matrix of a $\operatorname{BIBD}(6,5,4)$.

Let $A$ be the $6 \times 6$ circulant matrix whose first row is [010000]. Also let $B$ be the first 3 rows of the $6 \times 6$ circulant matrix whose first row is [010000]. Then the edge coloring incidence matrix of a $\operatorname{BIBD}(6,5,4)$ using 12 colors is given below.

$$
M_{i}=\begin{array}{|c|}
\hline\left(c_{1+4 i}\right) A^{2}+\left(c_{2+4 i}\right) A^{3}+\left(c_{3+4 i}\right) A^{4}+\left(c_{4+4 i}\right) A^{5} \\
\hline\left(c_{5+4 i}\right) A+\left(c_{6+4 i}\right) A^{3}+\left(c_{7+4 i}\right) A^{4}+\left(c_{8+4 i}\right) A^{5} \\
\hline\left(c_{9+4 i}\right) B+\left(c_{10+4 i}\right) B^{2}+\left(c_{11+4 i}\right) B^{4}+\left(c_{12+4 i}\right) B^{5} \\
\hline
\end{array}
$$

Since $M_{i}$ is semi-properly colored, by applying Theorem 2.12 we can properly color a $\operatorname{BIBD}(6,5, \lambda)$.

Lemma 4.5. There exists a properly colored $\operatorname{BIBD}(10,5, \lambda)$ for $\lambda=4 k, k \in \mathbb{Z}^{+}, k \geq 3$.

Proof: Figure 4.5 represents the edge-coloring incidence matrix for the $\operatorname{BIBD}(10,5,4)$. Let $A$ be the $9 \times 9$ circulant matrix whose first row is [010000000]. Then the edge coloring incidence matrix of a $\operatorname{BIBD}(10,5,4)$ using 12 colors is given below.

\begin{tabular}{|c|c|c|}
\hline \multirow{5}{*}{$M_{i}=$} & $\left(c_{4+4 i}\right) I$ & $\left(c_{2+4 i}\right) I+\left(c_{3+4 i}\right) A+\left(c_{1+4 i}\right) A^{8}$ \\
\hline & $\left(c_{5+4 i}\right) A^{5}$ & $\left(c_{7+4 i}\right) I+\left(c_{8+4 i}\right) A+\left(c_{6+4 i}\right) A^{7}$ \\
\hline & $\left(c_{9+4 i}\right) A^{3}+\left(c_{10+4 i}\right) A^{8}$ & $\left(c_{11+4 i}\right) A+\left(c_{12+4 i}\right) A^{8}$ \\
\hline & $\left(c_{2+4 i}\right) I+\left(c_{3+4 i}\right) A^{3}$ & $\left(c_{4+4 i}\right) I+\left(c_{5+4 i}\right) A^{5}$ \\
\hline & $\left(c_{1+4 i}\right) I+\left(c_{8+4 i}\right) A^{3}+\left(c_{6+4 i}\right) A^{5}+\left(c_{7+4 i}\right) A^{8}$ & 0 \\
\hline
\end{tabular}




\begin{tabular}{|c|c|c|c|c|c|c|c|c|c|c|c|c|c|c|c|c|c|c|}
\hline & $B_{1}$ & $B_{2}$ & $B_{3}$ & $B_{4}$ & $B_{5}$ & $B_{6}$ & $B_{7}$ & $B_{8}$ & $B_{9}$ & $B_{10}$ & $B_{11}$ & $B_{12}$ & $B_{13}$ & $B_{14}$ & $B_{15}$ & $B_{16}$ & $B_{17}$ & $B_{18}$ \\
\hline$\{0,1\}$ & $c_{4}$ & 0 & 0 & 0 & 0 & 0 & 0 & 0 & 0 & $c_{2}$ & $c_{3}$ & 0 & 0 & 0 & 0 & 0 & 0 & $c_{1}$ \\
\hline$\{1,2\}$ & 0 & $c_{4}$ & 0 & 0 & 0 & 0 & 0 & 0 & 0 & $c_{1}$ & $c_{2}$ & $c_{3}$ & 0 & 0 & 0 & 0 & 0 & 0 \\
\hline$\{2,3\}$ & 0 & 0 & $c_{4}$ & 0 & 0 & 0 & 0 & 0 & 0 & 0 & $c_{1}$ & $c_{2}$ & $c_{3}$ & 0 & 0 & 0 & 0 & 0 \\
\hline$\{3,4\}$ & 0 & 0 & 0 & $c_{4}$ & 0 & 0 & 0 & 0 & 0 & 0 & 0 & $c_{1}$ & $c_{2}$ & $c_{3}$ & 0 & 0 & 0 & 0 \\
\hline$\{4,5\}$ & 0 & 0 & 0 & 0 & $c_{4}$ & 0 & 0 & 0 & 0 & 0 & 0 & 0 & $c_{1}$ & $c_{2}$ & $c_{3}$ & 0 & 0 & 0 \\
\hline$\{5,6\}$ & 0 & 0 & 0 & 0 & 0 & $c_{4}$ & 0 & 0 & 0 & 0 & 0 & 0 & 0 & $c_{1}$ & $c_{2}$ & $c_{3}$ & 0 & 0 \\
\hline$\{6,7\}$ & 0 & 0 & 0 & 0 & 0 & 0 & $c_{4}$ & 0 & 0 & 0 & 0 & 0 & 0 & 0 & $c_{1}$ & $c_{2}$ & $c_{3}$ & 0 \\
\hline$\{7,8\}$ & 0 & 0 & 0 & 0 & 0 & 0 & 0 & $c_{4}$ & 0 & 0 & 0 & 0 & 0 & 0 & 0 & $c_{1}$ & $c_{2}$ & $c_{3}$ \\
\hline$\{8,0\}$ & 0 & 0 & 0 & 0 & 0 & 0 & 0 & 0 & $c_{4}$ & $c_{3}$ & 0 & 0 & 0 & 0 & 0 & 0 & $c_{1}$ & $c_{2}$ \\
\hline$\{0,2\}$ & 0 & 0 & 0 & 0 & 0 & $c_{5}$ & 0 & 0 & 0 & $c_{7}$ & $c_{8}$ & 0 & 0 & 0 & 0 & 0 & $c_{6}$ & 0 \\
\hline$\{1,3\}$ & 0 & 0 & 0 & 0 & 0 & 0 & $c_{5}$ & 0 & 0 & 0 & $c_{7}$ & $c_{8}$ & 0 & 0 & 0 & 0 & 0 & $c_{6}$ \\
\hline$\{2,4\}$ & 0 & 0 & 0 & 0 & 0 & 0 & 0 & $c_{5}$ & 0 & $c_{6}$ & 0 & $c_{7}$ & $c_{8}$ & 0 & 0 & 0 & 0 & 0 \\
\hline$\{3,5\}$ & 0 & 0 & 0 & 0 & 0 & 0 & 0 & 0 & $c_{5}$ & 0 & $c_{6}$ & 0 & $c_{7}$ & $c_{8}$ & 0 & 0 & 0 & 0 \\
\hline$\{4,6\}$ & $c_{5}$ & 0 & 0 & 0 & 0 & 0 & 0 & 0 & 0 & 0 & 0 & $c_{6}$ & 0 & $c_{7}$ & $c_{8}$ & 0 & 0 & 0 \\
\hline$\{5,7\}$ & 0 & $c_{5}$ & 0 & 0 & 0 & 0 & 0 & 0 & 0 & 0 & 0 & 0 & $c_{6}$ & 0 & $c_{7}$ & $c_{8}$ & 0 & 0 \\
\hline$\{6,8\}$ & 0 & 0 & $c_{5}$ & 0 & 0 & 0 & 0 & 0 & 0 & 0 & 0 & 0 & 0 & $c_{6}$ & 0 & $c_{7}$ & $c_{8}$ & 0 \\
\hline$\{7,0\}$ & 0 & 0 & 0 & $c_{5}$ & 0 & 0 & 0 & 0 & 0 & 0 & 0 & 0 & 0 & 0 & $c_{6}$ & 0 & $c_{7}$ & $c_{8}$ \\
\hline$\{8,1\}$ & 0 & 0 & 0 & 0 & $c_{5}$ & 0 & 0 & 0 & 0 & $c_{8}$ & 0 & 0 & 0 & 0 & 0 & $c_{6}$ & 0 & $c_{7}$ \\
\hline$\{0,3\}$ & 0 & 0 & 0 & $c_{10}$ & 0 & 0 & 0 & 0 & $c_{9}$ & 0 & $c_{11}$ & 0 & 0 & 0 & 0 & 0 & 0 & $c_{12}$ \\
\hline$\{1,4\}$ & $c_{9}$ & 0 & 0 & 0 & $c_{10}$ & 0 & 0 & 0 & 0 & $c_{12}$ & 0 & $c_{11}$ & 0 & 0 & 0 & 0 & 0 & 0 \\
\hline$\{2,5\}$ & 0 & $c_{9}$ & 0 & 0 & 0 & $c_{10}$ & 0 & 0 & 0 & 0 & $c_{12}$ & 0 & $c_{11}$ & 0 & 0 & 0 & 0 & 0 \\
\hline$\{3,6\}$ & 0 & 0 & $c_{9}$ & 0 & 0 & 0 & $c_{10}$ & 0 & 0 & 0 & 0 & $c_{12}$ & 0 & $c_{11}$ & 0 & 0 & 0 & 0 \\
\hline$\{4,7\}$ & 0 & 0 & 0 & $c_{9}$ & 0 & 0 & 0 & $c_{10}$ & 0 & 0 & 0 & 0 & $c_{12}$ & 0 & $c_{11}$ & 0 & 0 & 0 \\
\hline$\{5,8\}$ & 0 & 0 & 0 & 0 & $c_{9}$ & 0 & 0 & 0 & $c_{10}$ & 0 & 0 & 0 & 0 & $c_{12}$ & 0 & $c_{11}$ & 0 & 0 \\
\hline$\{6,0\}$ & $c_{10}$ & 0 & 0 & 0 & 0 & $c_{9}$ & 0 & 0 & 0 & 0 & 0 & 0 & 0 & 0 & $c_{12}$ & 0 & $c_{11}$ & 0 \\
\hline$\{7,1\}$ & 0 & $c_{10}$ & 0 & 0 & 0 & 0 & $c_{9}$ & 0 & 0 & 0 & 0 & 0 & 0 & 0 & 0 & $c_{12}$ & 0 & $c_{11}$ \\
\hline$\{8,2\}$ & 0 & 0 & $c_{10}$ & 0 & 0 & 0 & 0 & $c_{9}$ & 0 & $c_{11}$ & 0 & 0 & 0 & 0 & 0 & 0 & $c_{12}$ & 0 \\
\hline$\{0,4\}$ & $c_{2}$ & 0 & 0 & $c_{3}$ & 0 & 0 & 0 & 0 & 0 & $c_{4}$ & 0 & 0 & 0 & 0 & $c_{5}$ & 0 & 0 & 0 \\
\hline$\{1,5\}$ & 0 & $c_{2}$ & 0 & 0 & $c_{3}$ & 0 & 0 & 0 & 0 & 0 & $c_{4}$ & 0 & 0 & 0 & 0 & $c_{5}$ & 0 & 0 \\
\hline$\{2,6\}$ & 0 & 0 & $c_{2}$ & 0 & 0 & $c_{3}$ & 0 & 0 & 0 & 0 & 0 & $c_{4}$ & 0 & 0 & 0 & 0 & $c_{5}$ & 0 \\
\hline$\{3,7\}$ & 0 & 0 & 0 & $c_{2}$ & 0 & 0 & $c_{3}$ & 0 & 0 & 0 & 0 & 0 & $c_{4}$ & 0 & 0 & 0 & 0 & $c_{5}$ \\
\hline$\{4,8\}$ & 0 & 0 & 0 & 0 & $c_{2}$ & 0 & 0 & $c_{3}$ & 0 & $c_{5}$ & 0 & 0 & 0 & $c_{4}$ & 0 & 0 & 0 & 0 \\
\hline$\{5,0\}$ & 0 & 0 & 0 & 0 & 0 & $c_{2}$ & 0 & 0 & $c_{3}$ & 0 & $c_{5}$ & 0 & 0 & 0 & $c_{4}$ & 0 & 0 & 0 \\
\hline$\{6,1\}$ & $c_{3}$ & 0 & 0 & 0 & 0 & 0 & $c_{2}$ & 0 & 0 & 0 & 0 & $c_{5}$ & 0 & 0 & 0 & $c_{4}$ & 0 & 0 \\
\hline$\{7,2\}$ & 0 & $c_{3}$ & 0 & 0 & 0 & 0 & 0 & $c_{2}$ & 0 & 0 & 0 & 0 & $c_{5}$ & 0 & 0 & 0 & $c_{4}$ & 0 \\
\hline$\{8,3\}$ & 0 & 0 & $c_{3}$ & 0 & 0 & 0 & 0 & 0 & $c_{2}$ & 0 & 0 & 0 & 0 & $c_{5}$ & 0 & 0 & 0 & $c_{4}$ \\
\hline$\{\infty, 0\}$ & $c_{1}$ & 0 & 0 & $c_{8}$ & 0 & $c_{6}$ & 0 & 0 & $c_{7}$ & 0 & 0 & 0 & 0 & 0 & 0 & 0 & 0 & 0 \\
\hline$\{\infty, 1\}$ & $c_{7}$ & $c_{1}$ & 0 & 0 & $c_{8}$ & 0 & $c_{6}$ & 0 & 0 & 0 & 0 & 0 & 0 & 0 & 0 & 0 & 0 & 0 \\
\hline$\{\infty, 2\}$ & 0 & $c_{7}$ & $c_{1}$ & 0 & 0 & $c_{8}$ & 0 & $c_{6}$ & 0 & 0 & 0 & 0 & 0 & 0 & 0 & 0 & 0 & 0 \\
\hline$\{\infty, 3\}$ & 0 & 0 & $c_{7}$ & $c_{1}$ & 0 & 0 & $c_{8}$ & 0 & $c_{6}$ & 0 & 0 & 0 & 0 & 0 & 0 & 0 & 0 & 0 \\
\hline$\{\infty, 4\}$ & $c_{6}$ & 0 & 0 & $c_{7}$ & $c_{1}$ & 0 & 0 & $c_{8}$ & 0 & 0 & 0 & 0 & 0 & 0 & 0 & 0 & 0 & 0 \\
\hline$\{\infty, 5\}$ & 0 & $c_{6}$ & 0 & 0 & $c_{7}$ & $c_{1}$ & 0 & 0 & $c_{8}$ & 0 & 0 & 0 & 0 & 0 & 0 & 0 & 0 & 0 \\
\hline$\{\infty, 6\}$ & $c_{8}$ & 0 & $c_{6}$ & 0 & 0 & $c_{7}$ & $c_{1}$ & 0 & 0 & 0 & 0 & 0 & 0 & 0 & 0 & 0 & 0 & 0 \\
\hline$\{\infty, 7\}$ & 0 & $c_{8}$ & 0 & $c_{6}$ & 0 & 0 & $c_{7}$ & $c_{1}$ & 0 & 0 & 0 & 0 & 0 & 0 & 0 & 0 & 0 & 0 \\
\hline$\{\infty, 8\}$ & 0 & 0 & $c_{8}$ & 0 & $c_{6}$ & 0 & 0 & $c_{7}$ & $c_{1}$ & 0 & 0 & 0 & 0 & 0 & 0 & 0 & 0 & 0 \\
\hline
\end{tabular}

Figure 4.5: Edge-Coloring Incidence Matrix of a $\operatorname{BIBD}(10,5,4)$. 
Since $M_{i}$ is semi-properly colored, by applying Theorem 2.12 we can properly color a $\operatorname{BIBD}(10,5, \lambda)$.

Lemma 4.6. There exists a properly colored $\operatorname{BIBD}(20,5, \lambda)$ for $\lambda=4 k, k \in \mathbb{Z}^{+}, k \geq 3$.

Proof: Let $A$ be the $19 \times 19$ circulant matrix whose first row is [0100000000000000000]. Then the edge-coloring incidence matrix of a $\operatorname{BIBD}(20,5,4)$ using 12 colors in Figure 4.6

\begin{tabular}{|c|c|c|c|}
\hline$\left(c_{4+4 i}\right) A^{17}$ & $\left(c_{3+4 i}\right) A^{14}$ & $\left(c_{2+4 i}\right) A^{12}$ & $\left(c_{1+4 i}\right) A^{2}$ \\
\hline$\left(c_{1+4 i}\right) I$ & $\left(c_{2+4 i}\right) A^{9}$ & 0 & $\left(c_{3+4 i}\right) A^{4}+\left(c_{4+4 i}\right) A^{10}$ \\
\hline$\left(c_{3+4 i}\right) I$ & 0 & $\left(c_{1+4 i}\right) A^{6}+\left(c_{4+4 i}\right) A^{15}$ & $\left(c_{2+4 i}\right) A^{4}$ \\
\hline 0 & $\left(c_{1+4 i}\right) A^{13}+\left(c_{4+4 i}\right) A^{18}$ & $\left(c_{3+4 i}\right) A^{15}$ & $\left(c_{6+4 i}\right) A^{8}$ \\
\hline$\left(c_{2+4 i}\right) A^{5}$ & $\left(c_{5+4 i}\right) A^{14}+\left(c_{8+4 i}\right) A^{18}$ & $\left(c_{7+4 i}\right) A^{11}$ & 0 \\
\hline 0 & $\left(c_{6+4 i}\right) A^{13}$ & $\left(c_{8+4 i}\right) A^{11}$ & $\left(c_{5+2 i}\right) A^{8}+\left(c_{7+4 i}\right) A^{10}$ \\
\hline$\left(c_{6+4 i}\right) A^{5}$ & $\left(c_{7+4 i}\right) A^{14}$ & $\left(c_{5+4 i}\right) A^{3}$ & $\left(c_{8+4 i}\right) A^{8}$ \\
\hline$\left(c_{5+4 i}\right) A^{5}$ & $\left(c_{10+4 i}\right) A^{7}$ & $\left(c_{11+4 i}\right) A^{11}$ & $\left(c_{12+4 i}\right) A^{10}$ \\
\hline 0 & $\left(c_{9+4 i}\right) A^{18}$ & $\left(c_{10+4 i}\right) A^{12}+\left(c_{12+4 i}\right) A^{15}$ & $\left(c_{11+4 i}\right) A^{10}$ \\
\hline & 0 & 0 & 0 \\
\hline $\begin{array}{c}\left(c_{7+4 i}\right) I+\left(c_{8+4 i}\right) A^{5}+ \\
\left(c_{9+4 i}\right) A^{16}+\left(c_{10+4 i}\right) A^{17}\end{array}$ & 0 & 0 & 0 \\
\hline
\end{tabular}

Figure 4.6: Edge-Coloring Incidence Matrix of a $\operatorname{BIBD}(20,5,4)$.

Since $M_{i}$ is semi-properly colored, by applying Theorem 2.12 we can properly color a $\operatorname{BIBD}(20,5, \lambda)$.

Lemma 4.7. There exists a properly colored $\operatorname{BIBD}(36,5, \lambda)$ for $\lambda=4 k, k \in \mathbb{Z}^{+}, k \geq 3$.

Proof: Let $A$ be the $36 \times 36$ circulant matrix whose first row is

$$
\text { [01000000000000000000000000000000000]. }
$$

Let $B$ be the first 18 rows of a $36 \times 36$ circulant matrix whose first row is

$$
\text { [01000000000000000000000000000000000]. }
$$

Then the edge-coloring incidence matrix of a $\operatorname{BIBD}(36,5,4)$ using 12 colors in Figure 4.7

Since $M_{i}$ is semi-properly colored, by applying Theorem 2.12 we can properly color a $\operatorname{BIBD}(36,5, \lambda)$.

Lemma 4.8. There exists a properly colored $\operatorname{BIBD}(40,5, \lambda)$ for $\lambda=4 k, k \in \mathbb{Z}^{+}, k \geq 3$.

Proof: Let $A$ be the $39 \times 39$ circulant matrix whose first row is

$$
\text { [010000000000000000000000000000000000000]. }
$$

Then the edge coloring incidence matrix of a $\operatorname{BIBD}(40,5,4)$ using 12 colors in Figure 4.8

Since $M_{i}$ is semi-properly colored, by applying Theorem 2.12 we can properly color a $\operatorname{BIBD}(40,5, \lambda)$. 


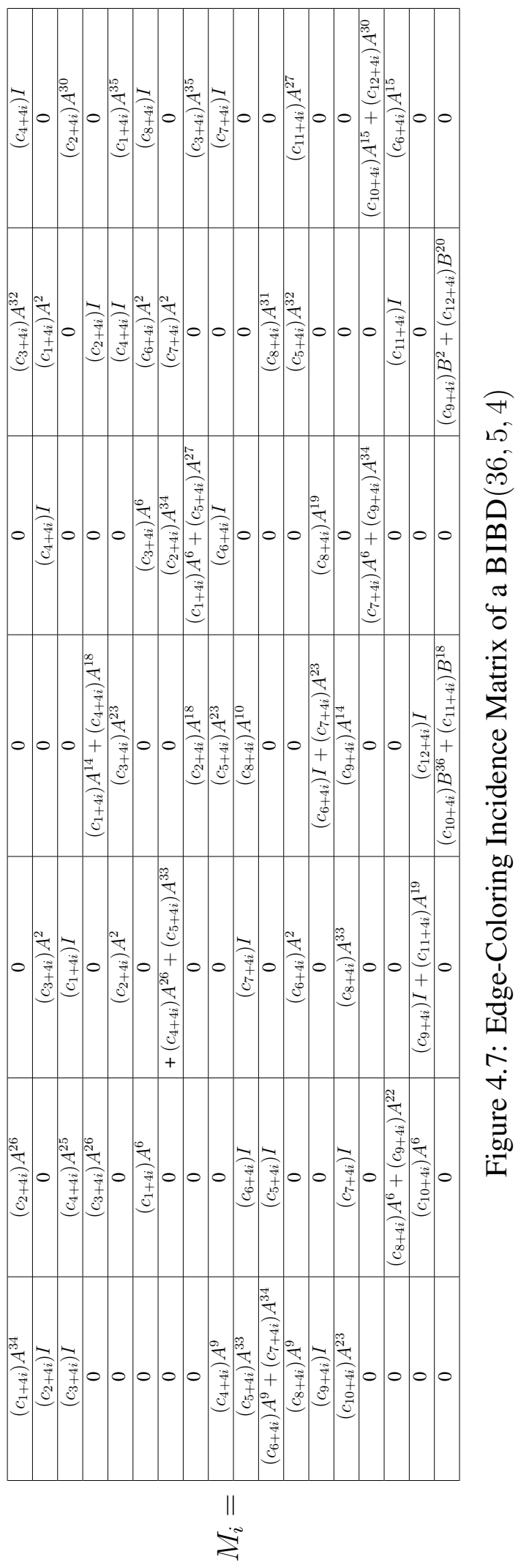




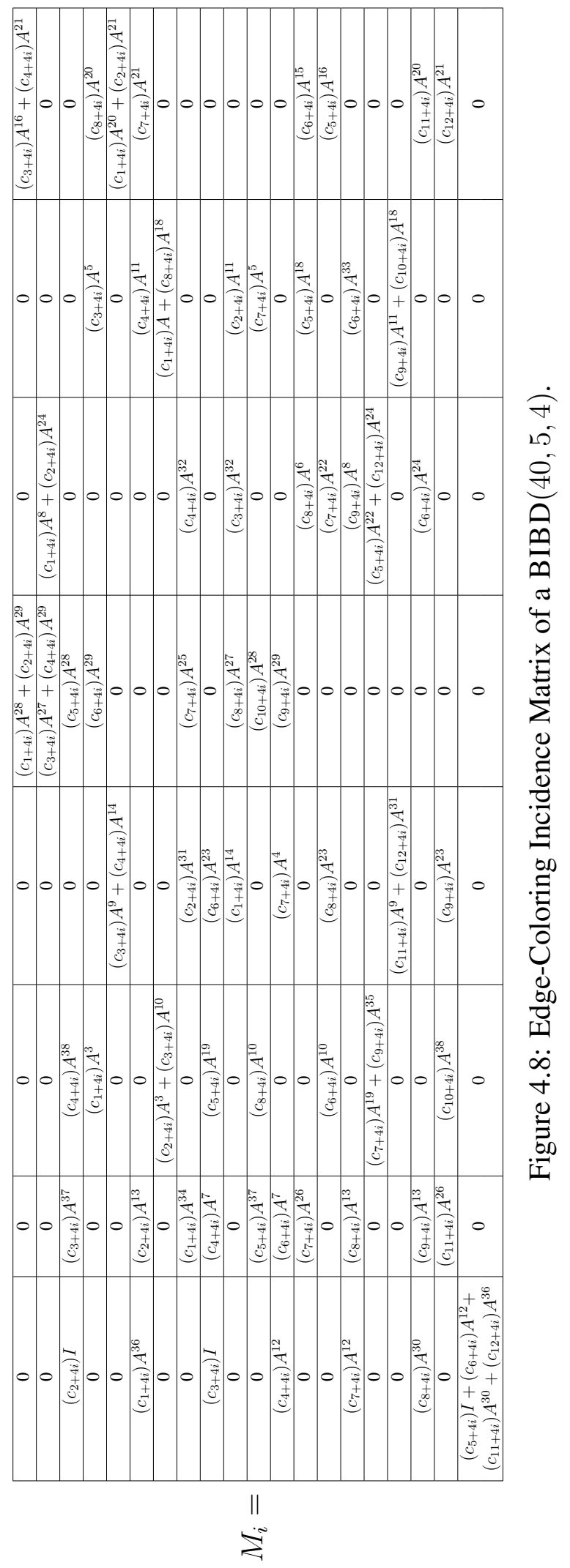


Lemma 4.9. There exists a properly colored $\operatorname{BIBD}(56,5, \lambda)$ for $\lambda=4 k, k \in \mathbb{Z}^{+}, k \geq 3$.

Proof: Let $A$ be the $56 \times 56$ circulant matrix whose first row is

\section{[0100000000000000000000000000000000000000000000000000000].}

Let $B$ be the first 28 rows of a $56 \times 56$ circulant matrix whose first row is

$$
\text { [0100000000000000000000000000000000000000000000000000000]. }
$$

Then the edge coloring incidence matrix of $\operatorname{a\operatorname {IBD}}(56,5,4)$ using 12 colors in Figures 4.9 and 4.10 .

Since the concatenation of $M_{i}$ and $N_{i}$ is semi-properly colored, by Theorem 2.12 we can properly color a $\operatorname{BIBD}(56,5, \lambda)$.

Lemma 4.10. There exists a properly colored $\operatorname{BIBD}(15,5, \lambda)$ for $\lambda=2 k, k \in \mathbb{Z}^{+}, k \geq 5$.

Proof: Note that the colors $c_{i}$ and $d_{i}$ are distinct for all $i$. We form an edge-coloring incidence matrix for $\operatorname{a\operatorname {BID}}(15,5, \lambda)$ using $\operatorname{B} \operatorname{BIBD}(15,5,4)$ and $\mathrm{a} \operatorname{BIBD}(15,5,6)$ as follows. Let $A$ be the $14 \times 14$ circulant matrix whose first row is [01000000000000]. Let $B$ be the first 7 rows of a $14 \times 14$ circulant matrix whose first row is [01000000000000]

Then the edge coloring incidence matrix of a $\operatorname{BIBD}(15,5,4)$ using 12 colors is given below.

\begin{tabular}{|c|c|c|c|}
\hline \multirow{8}{*}{$M_{i}=$} & $\left(c_{1+4 i}\right) I$ & $\left(c_{2+4 i}\right) I+\left(c_{3+4 i}\right) A^{9}+\left(c_{4+4 i}\right) A^{13}$ & 0 \\
\hline & $\left(c_{2+4 i}\right) A^{13}$ & $\left(c_{1+4 i}\right) I$ & $\left(c_{3+4 i}\right) I+\left(c_{4+4 i}\right) A^{12}$ \\
\hline & $\left(c_{3+4 i}\right) I$ & $\left(c_{8+4 i}\right) A^{12}$ & $\left(c_{1+4 i}\right) A^{7}+\left(c_{2+4 i}\right) A^{10}$ \\
\hline & 0 & $\left(c_{5+4 i}\right) A^{12}+\left(c_{6+4 i}\right) A^{13}$ & $\left(c_{7+4 i}\right) I+\left(c_{8+4 i}\right) A^{4}$ \\
\hline & $\left(c_{4+4 i}\right) A^{11}$ & $\left(c_{7+4 i}\right) I+\left(c_{9+4 i}\right) A^{13}$ & $\left(c_{6+4 i}\right) A^{12}$ \\
\hline & $\left(c_{5+4 i}\right) A^{6}$ & $\left(c_{10+4 i}\right) I$ & $\left(c_{11+4 i}\right) A^{4}+\left(c_{12+4 i}\right) A^{10}$ \\
\hline & $\left(c_{11+4 i}\right) B^{6}+\left(c_{12+4 i}\right) B^{13}$ & 0 & $\left(c_{9+4 i}\right) I+\left(c_{10+4 i}\right) B^{7}$ \\
\hline & $\left(c_{7+4 i}\right) I+\left(c_{8+4 i}\right) A^{6}+\left(c_{9+4 i}\right) A^{11}+\left(c_{10+4 i}\right) A^{13}$ & 0 & 0 \\
\hline
\end{tabular}

Let $C$ be the $15 \times 15$ circulant matrix whose first row is [010000000000000]. Let $E_{i}^{(j)}$ be the following $15 \times 3$ matrix.

$$
\left.E_{i}^{(1)}=\begin{array}{ccc}
d_{7+6 i} & 0 & 0 \\
0 & d_{7+6 i} & 0 \\
0 & 0 & d_{7+6 i} \\
d_{8+6 i} & 0 & 0 \\
0 & d_{8+6 i} & 0 \\
0 & 0 & d_{8+6 i} \\
d_{9+6 i} & 0 & 0 \\
0 & d_{9+6 i} & 0 \\
0 & 0 & d_{9+6 i} \\
d_{10+6 i} & 0 & 0 \\
0 & d_{10+6 i} & 0 \\
0 & 0 & d_{10+6 i} \\
d_{11+6 i} & 0 & 0 \\
0 & d_{11+6 i} & 0 \\
0 & 0 & d_{11+6 i}
\end{array}\right] E_{i}^{(2)}=\begin{array}{ccc}
d_{4+6 i} & 0 & 0 \\
0 & d_{4+6 i} & 0 \\
0 & 0 & d_{4+6 i} \\
d_{12+6 i} & 0 & 0 \\
0 & d_{12+6 i} & 0 \\
0 & 0 & d_{12+6 i} \\
d_{5+6 i} & 0 & 0 \\
0 & d_{5+6 i} & 0 \\
0 & 0 & d_{5+6 i} \\
d_{6+6 i} & 0 & 0 \\
0 & d_{6+6 i} & 0 \\
0 & 0 & d_{6+6 i} \\
d_{3+6 i} & 0 & 0 \\
0 & d_{3+6 i} & 0 \\
0 & 0 & d_{3+6 i} \\
\hline
\end{array}
$$




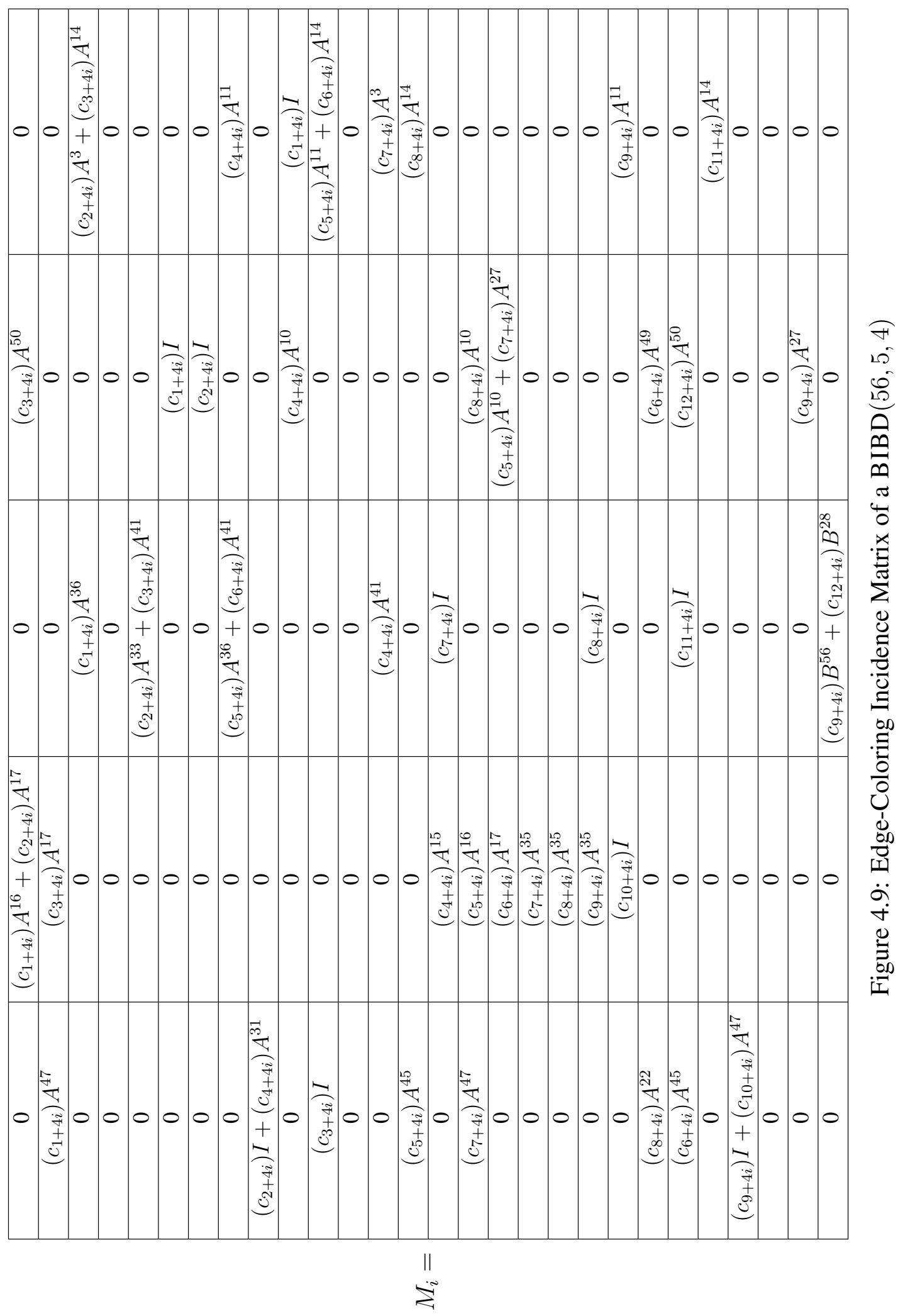




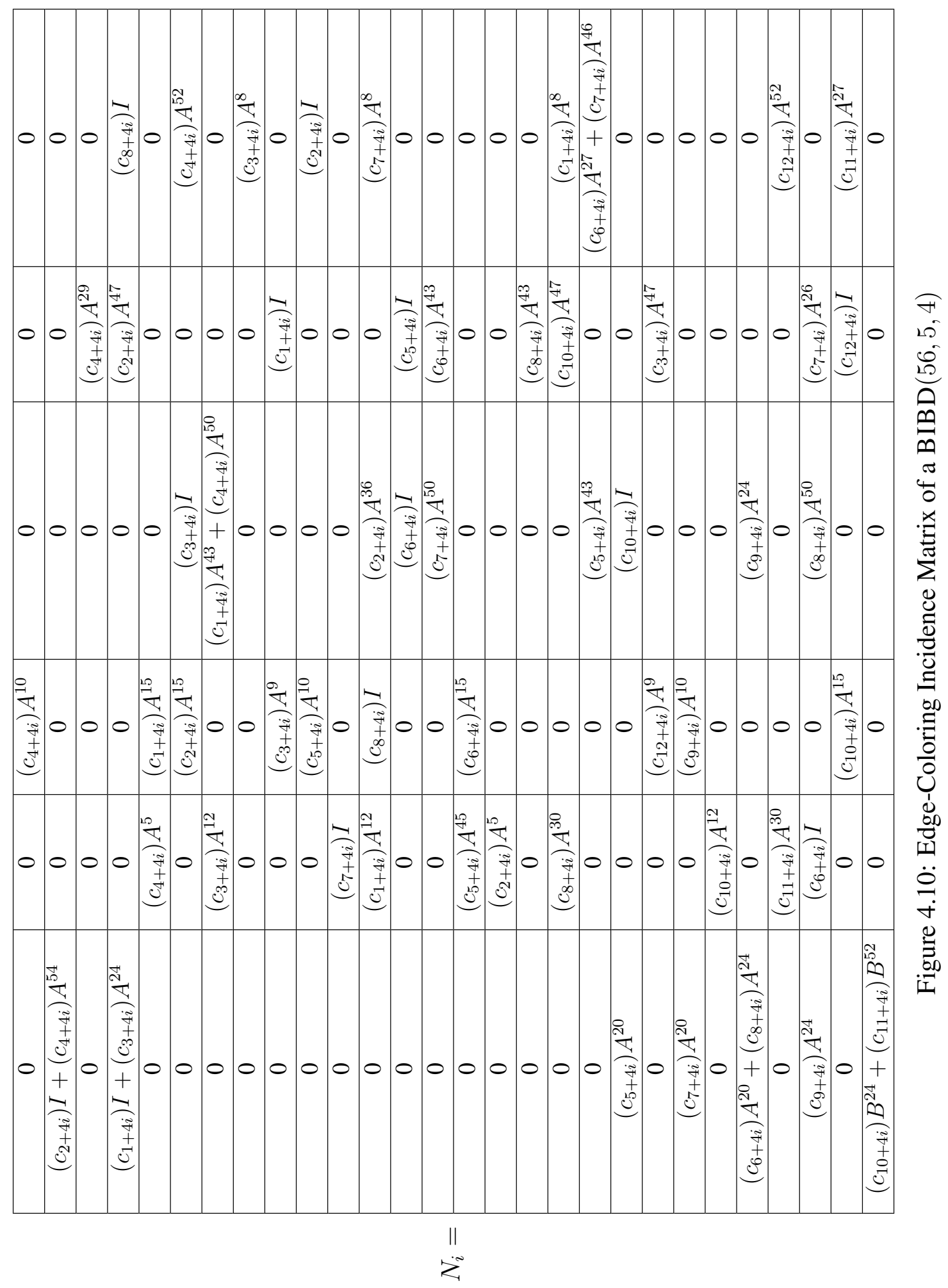


Let $F_{i}^{(j)}$ for $j=1,2,3,4,5,6$ be the following $15 \times 15$ matrix.

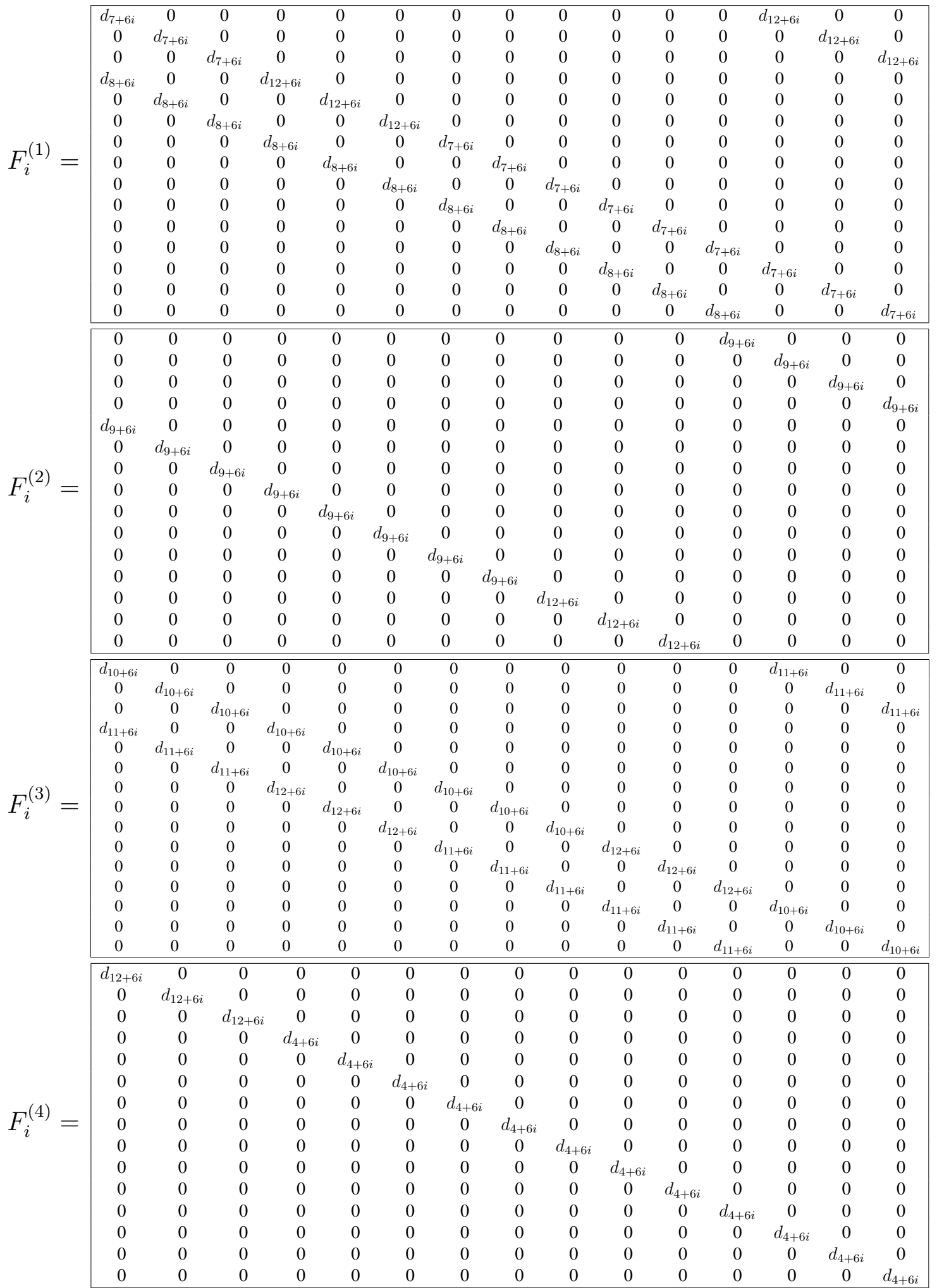




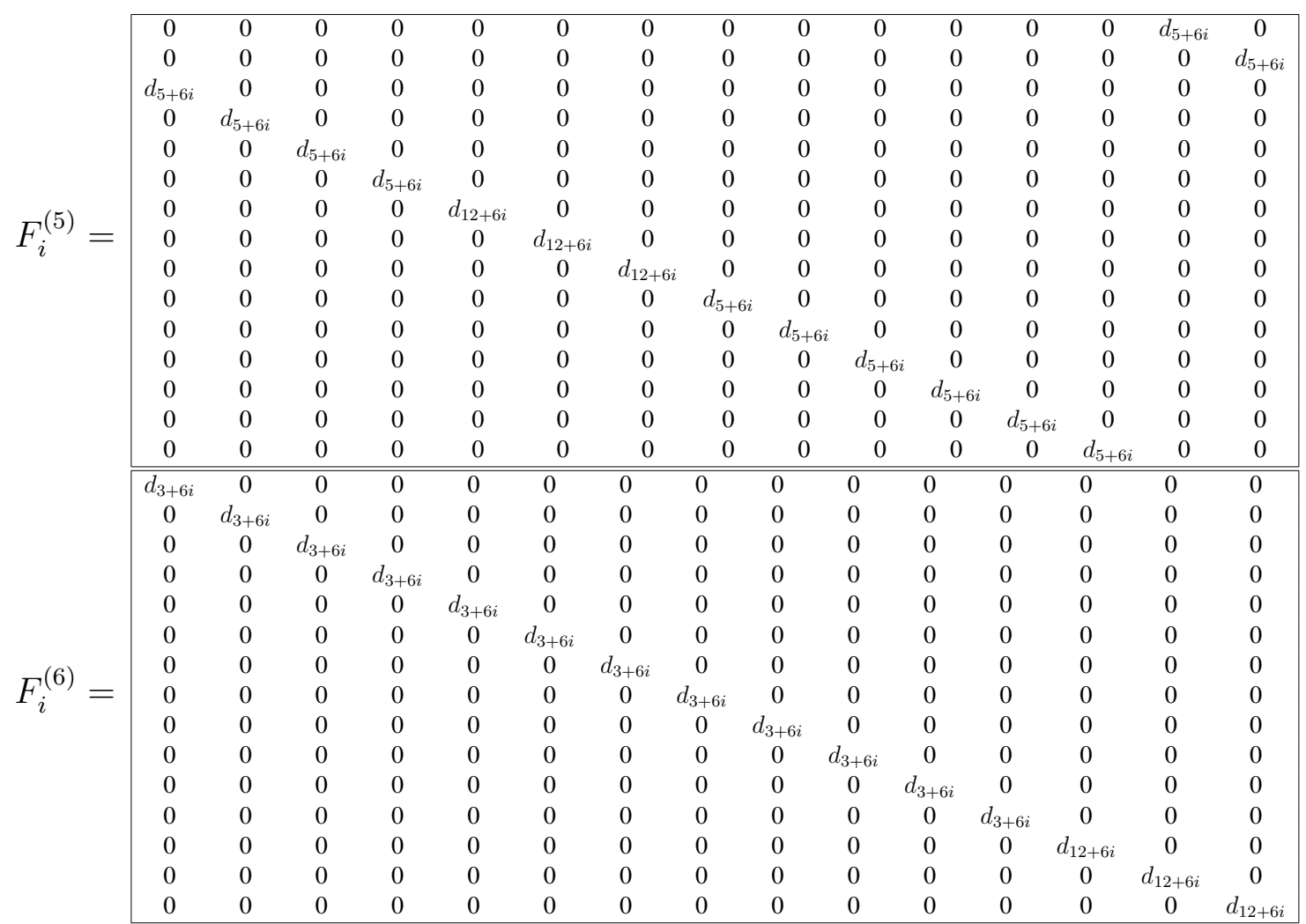

Thus, the edge-coloring incidence matrix of a $\operatorname{BIBD}(15,5,6)$ using 12 colors is given Figure 4.11.

Suppose $k \equiv 0(\bmod 2)$. To form a properly colored $\operatorname{BIBD}(15,5, \lambda)$ with $\lambda=2 k, k \in$ $\mathbb{Z}^{+}, k \geq 5$, we simply repeat the blocks of the $\operatorname{BIBD}(15,5,4) \frac{k}{2}$ times and follow the same coloring scheme with different colors. The subscripts of the colors are all computed $(\bmod \lambda)$ where we identify $c_{0}$ with $c_{\lambda}$. Thus the edge-coloring incidence matrix of a properly colored $\operatorname{BIBD}(15,5, \lambda)$ for $k \equiv 0(\bmod 2)$ can be given in terms of the $M_{i}$ as

$$
M=\begin{array}{|l|l|l|l|}
\hline M_{0} & M_{1} & \cdots & M_{\frac{k}{2}-1} \\
\hline
\end{array}
$$

Suppose $k \equiv 1(\bmod 2)$. To form a properly colored $\operatorname{BIBD}(15,5, \lambda)$ with $\lambda=2 k, k \in$ $\mathbb{Z}^{+}, k \geq 13$, we simply repeat the blocks of the $\operatorname{BIBD}(15,5,4) \frac{\lambda-6}{4}$ times joined with the blocks of a $\operatorname{BIBD}(15,5,6)$. We know we can properly color a $\operatorname{BIBD}(15,5,10)$ by Theorem 2.2. The colors used on the $\operatorname{BIBD}(15,5,4)$ and $\operatorname{BIBD}(15,5,6)$ will be distinct from each other requiring $\lambda \geq 26$. The subscripts of the colors are all computed $(\bmod \lambda)$ where we identify $c_{0}$ with $c_{\lambda}$. Thus the edge coloring incidence matrix of a properly colored $\operatorname{BIBD}(15,5, \lambda)$ for $13 \leq k \equiv 1(\bmod 2)$ can be given in terms of the $M_{i}$ as

$$
M=\begin{array}{|l|l|l|l|l|}
\hline M_{\alpha_{0}} & M_{0} & M_{1} & \cdots & M_{((\lambda-6) / 4)-1} . \\
\hline
\end{array}
$$

This leaves three cases where $\lambda=14,18,22$. Suppose $\lambda=14$. We form an edgecoloring incidence matrix using 14 colors for a $\operatorname{BIBD}(15,5,14)$. Let $H$ be a $15 \times 15$ circulant matrix whose first row is [010000000000000]. Let $G^{(j)}$ for $j=1,2$ be the following $15 \times 3$ matrices. 


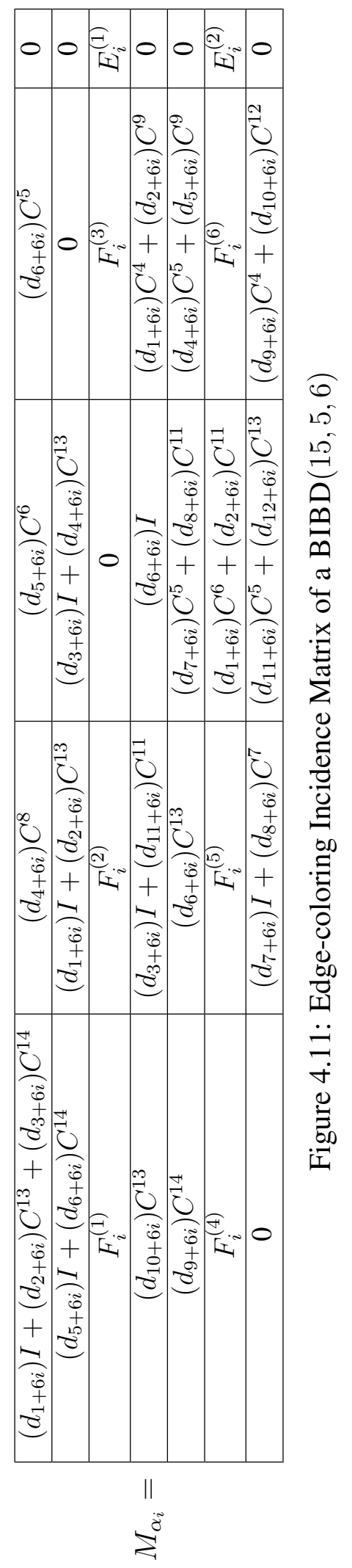




$$
\left.G^{(1)}=\begin{array}{ccc}
c_{1} & 0 & 0 \\
0 & c_{1} & 0 \\
0 & 0 & c_{1} \\
c_{3} & 0 & 0 \\
0 & c_{3} & 0 \\
0 & 0 & c_{3} \\
c_{12} & 0 & 0 \\
0 & c_{12} & 0 \\
0 & 0 & c_{12} \\
c_{13} & 0 & 0 \\
0 & c_{13} & 0 \\
0 & 0 & c_{13} \\
c_{14} & 0 & 0 \\
0 & c_{14} & 0 \\
0 & 0 & c_{14}
\end{array}\right] G^{(2)}=\left[\begin{array}{ccc}
c_{4} & 0 & 0 \\
0 & c_{4} & 0 \\
0 & 0 & c_{4} \\
c_{5} & 0 & 0 \\
0 & c_{5} & 0 \\
0 & 0 & c_{5} \\
c_{6} & 0 & 0 \\
0 & c_{6} & 0 \\
0 & 0 & c_{6} \\
c_{8} & 0 & 0 \\
0 & c_{8} & 0 \\
0 & 0 & c_{8} \\
c_{9} & 0 & 0 \\
0 & c_{9} & 0 \\
0 & 0 & c_{9}
\end{array}\right.
$$

Let $G^{(j)}$ for $j=3,4$ be the following $15 \times 15$ matrices.

$\left.G^{(3)}=\begin{array}{cccccccccccccccc}0 & 0 & 0 & 0 & 0 & 0 & 0 & 0 & 0 & 0 & 0 & 0 & 0 & c_{3} & 0 \\ 0 & 0 & 0 & 0 & 0 & 0 & 0 & 0 & 0 & 0 & 0 & 0 & 0 & 0 & c_{3} \\ c_{3} & 0 & 0 & 0 & 0 & 0 & 0 & 0 & 0 & 0 & 0 & 0 & 0 & 0 & 0 \\ 0 & c_{12} & 0 & 0 & 0 & 0 & 0 & 0 & 0 & 0 & 0 & 0 & 0 & 0 & 0 \\ 0 & 0 & c_{12} & 0 & 0 & 0 & 0 & 0 & 0 & 0 & 0 & 0 & 0 & 0 & 0 \\ 0 & 0 & 0 & c_{12} & 0 & 0 & 0 & 0 & 0 & 0 & 0 & 0 & 0 & 0 & 0 \\ 0 & 0 & 0 & 0 & c_{13} & 0 & 0 & 0 & 0 & 0 & 0 & 0 & 0 & 0 & 0 \\ 0 & 0 & 0 & 0 & 0 & c_{13} & 0 & 0 & 0 & 0 & 0 & 0 & 0 & 0 & 0 \\ 0 & 0 & 0 & 0 & 0 & 0 & c_{13} & 0 & 0 & 0 & 0 & 0 & 0 & 0 & 0 \\ 0 & 0 & 0 & 0 & 0 & 0 & 0 & c_{14} & 0 & 0 & 0 & 0 & 0 & 0 & 0 \\ 0 & 0 & 0 & 0 & 0 & 0 & 0 & 0 & c_{14} & 0 & 0 & 0 & 0 & 0 & 0 \\ 0 & 0 & 0 & 0 & 0 & 0 & 0 & 0 & 0 & c_{14} & 0 & 0 & 0 & 0 & 0 \\ 0 & 0 & 0 & 0 & 0 & 0 & 0 & 0 & 0 & 0 & c_{1} & 0 & 0 & 0 & 0 \\ 0 & 0 & 0 & 0 & 0 & 0 & 0 & 0 & 0 & 0 & 0 & c_{1} & 0 & 0 & 0 \\ 0 & 0 & 0 & 0 & 0 & 0 & 0 & 0 & 0 & 0 & 0 & 0 & c_{1} & 0 & 0\end{array}\right]$




$G^{(4)}=$\begin{tabular}{cccccccccccccccc}
0 & 0 & 0 & 0 & 0 & 0 & 0 & 0 & 0 & 0 & 0 & 0 & 0 & 0 & $c_{9}$ \\
$c_{9}$ & 0 & 0 & 0 & 0 & 0 & 0 & 0 & 0 & 0 & 0 & 0 & 0 & 0 & 0 \\
0 & $c_{9}$ & 0 & 0 & 0 & 0 & 0 & 0 & 0 & 0 & 0 & 0 & 0 & 0 & 0 \\
0 & 0 & $c_{4}$ & 0 & 0 & 0 & 0 & 0 & 0 & 0 & 0 & 0 & 0 & 0 & 0 \\
0 & 0 & 0 & $c_{4}$ & 0 & 0 & 0 & 0 & 0 & 0 & 0 & 0 & 0 & 0 & 0 \\
0 & 0 & 0 & 0 & $c_{4}$ & 0 & 0 & 0 & 0 & 0 & 0 & 0 & 0 & 0 & 0 \\
0 & 0 & 0 & 0 & 0 & $c_{5}$ & 0 & 0 & 0 & 0 & 0 & 0 & 0 & 0 & 0 \\
0 & 0 & 0 & 0 & 0 & 0 & $c_{5}$ & 0 & 0 & 0 & 0 & 0 & 0 & 0 & 0 \\
0 & 0 & 0 & 0 & 0 & 0 & 0 & $c_{5}$ & 0 & 0 & 0 & 0 & 0 & 0 & 0 \\
0 & 0 & 0 & 0 & 0 & 0 & 0 & 0 & $c_{6}$ & 0 & 0 & 0 & 0 & 0 & 0 \\
0 & 0 & 0 & 0 & 0 & 0 & 0 & 0 & 0 & $c_{6}$ & 0 & 0 & 0 & 0 & 0 \\
0 & 0 & 0 & 0 & 0 & 0 & 0 & 0 & 0 & 0 & $c_{6}$ & 0 & 0 & 0 & 0 \\
0 & 0 & 0 & 0 & 0 & 0 & 0 & 0 & 0 & 0 & 0 & $c_{8}$ & 0 & 0 & 0 \\
0 & 0 & 0 & 0 & 0 & 0 & 0 & 0 & 0 & 0 & 0 & 0 & $c_{8}$ & 0 & 0 \\
0 & 0 & 0 & 0 & 0 & 0 & 0 & 0 & 0 & 0 & 0 & 0 & 0 & $c_{8}$ & 0 \\
\hline
\end{tabular}

Let $G^{(j)}$ for $j=5,6$ be the following $15 \times 9$ matrices.

$G^{(5)}=$\begin{tabular}{ccccccccc}
$c_{14}$ & 0 & 0 & $c_{13}$ & 0 & 0 & $c_{12}$ & 0 & 0 \\
0 & $c_{14}$ & 0 & 0 & $c_{13}$ & 0 & 0 & $c_{12}$ & 0 \\
0 & 0 & $c_{14}$ & 0 & 0 & $c_{13}$ & 0 & 0 & $c_{12}$ \\
$c_{1}$ & 0 & 0 & $c_{14}$ & 0 & 0 & $c_{13}$ & 0 & 0 \\
0 & $c_{1}$ & 0 & 0 & $c_{14}$ & 0 & 0 & $c_{13}$ & 0 \\
0 & 0 & $c_{1}$ & 0 & 0 & $c_{14}$ & 0 & 0 & $c_{13}$ \\
$c_{3}$ & 0 & 0 & $c_{1}$ & 0 & 0 & $c_{14}$ & 0 & 0 \\
0 & $c_{3}$ & 0 & 0 & $c_{1}$ & 0 & 0 & $c_{14}$ & 0 \\
0 & 0 & $c_{3}$ & 0 & 0 & $c_{1}$ & 0 & 0 & $c_{14}$ \\
$c_{12}$ & 0 & 0 & $c_{3}$ & 0 & 0 & $c_{1}$ & 0 & 0 \\
0 & $c_{12}$ & 0 & 0 & $c_{3}$ & 0 & 0 & $c_{1}$ & 0 \\
0 & 0 & $c_{12}$ & 0 & 0 & $c_{3}$ & 0 & 0 & $c_{1}$ \\
$c_{13}$ & 0 & 0 & $c_{12}$ & 0 & 0 & $c_{3}$ & 0 & 0 \\
0 & $c_{13}$ & 0 & 0 & $c_{12}$ & 0 & 0 & $c_{3}$ & 0 \\
0 & 0 & $c_{13}$ & 0 & 0 & $c_{12}$ & 0 & 0 & $c_{3}$ \\
\hline
\end{tabular}




$G^{(6)}=$\begin{tabular}{ccccccccc}
$c_{8}$ & 0 & 0 & $c_{6}$ & 0 & 0 & $c_{5}$ & 0 & 0 \\
0 & $c_{8}$ & 0 & 0 & $c_{6}$ & 0 & 0 & $c_{5}$ & 0 \\
0 & 0 & $c_{8}$ & 0 & 0 & $c_{6}$ & 0 & 0 & $c_{5}$ \\
$c_{9}$ & 0 & 0 & $c_{8}$ & 0 & 0 & $c_{6}$ & 0 & 0 \\
0 & $c_{9}$ & 0 & 0 & $c_{8}$ & 0 & 0 & $c_{6}$ & 0 \\
0 & 0 & $c_{9}$ & 0 & 0 & $c_{8}$ & 0 & 0 & $c_{6}$ \\
$c_{4}$ & 0 & 0 & $c_{9}$ & 0 & 0 & $c_{8}$ & 0 & 0 \\
0 & $c_{4}$ & 0 & 0 & $c_{9}$ & 0 & 0 & $c_{8}$ & 0 \\
0 & 0 & $c_{4}$ & 0 & 0 & $c_{9}$ & 0 & 0 & $c_{8}$ \\
$c_{5}$ & 0 & 0 & $c_{4}$ & 0 & 0 & $c_{9}$ & 0 & 0 \\
0 & $c_{5}$ & 0 & 0 & $c_{4}$ & 0 & 0 & $c_{9}$ & 0 \\
0 & 0 & $c_{5}$ & 0 & 0 & $c_{4}$ & 0 & 0 & $c_{9}$ \\
$c_{6}$ & 0 & 0 & $c_{5}$ & 0 & 0 & $c_{4}$ & 0 & 0 \\
0 & $c_{6}$ & 0 & 0 & $c_{5}$ & 0 & 0 & $c_{4}$ & 0 \\
0 & 0 & $c_{6}$ & 0 & 0 & $c_{5}$ & 0 & 0 & $c_{4}$ \\
\hline
\end{tabular}

Thus the edge-coloring incidence matrix of a properly colored $\operatorname{BIBD}(15,5,14)$ is given by concatenating $N^{(0)}$ and $N^{(1)}$ from Figure 4.12 .

Now suppose $\lambda=18$. All subscripts are computed (mod 18). We form the properly colored $\operatorname{BIBD}(15,5,18)$ given in terms of the $M_{\alpha_{i}}$ as

$$
M=\begin{array}{|l|l|l|}
\hline M_{\alpha_{0}} & M_{\alpha_{1}} & M_{\alpha_{2}} \\
\hline
\end{array}
$$

Now suppose $\lambda=22$. Let $M_{0^{\prime}}$ be the properly colored $\operatorname{BIBD}(15,5,10)$ on 10 colors. Then

$$
M=\begin{array}{|l|l|l|l|}
\hline M_{0^{\prime}} & M_{0} & M_{1} & M_{2} \\
\hline
\end{array}
$$

Lemma 4.11. There exists a properly colored $\operatorname{BIBD}(35,5, \lambda)$ for $\lambda=2 k, k \in \mathbb{Z}^{+}, k \geq 5$.

Proof: Let $A$ be the $35 \times 35$ circulant matrix whose first row is

$$
\text { [01000000000000000000000000000000000]. }
$$

Let $A_{i}^{(j)}$ be the following $35 \times 28$ matrices for $j=1,2$ in Figures 4.13 and 4.14 


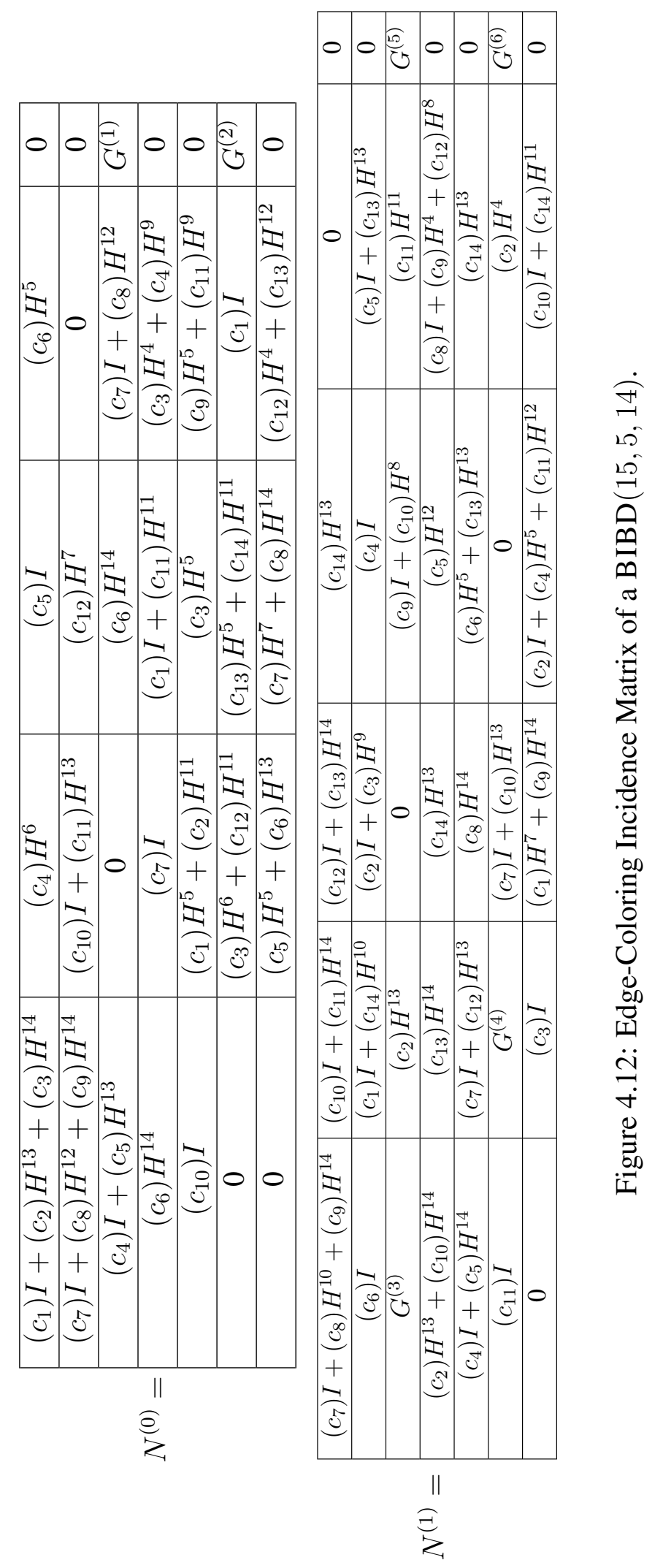




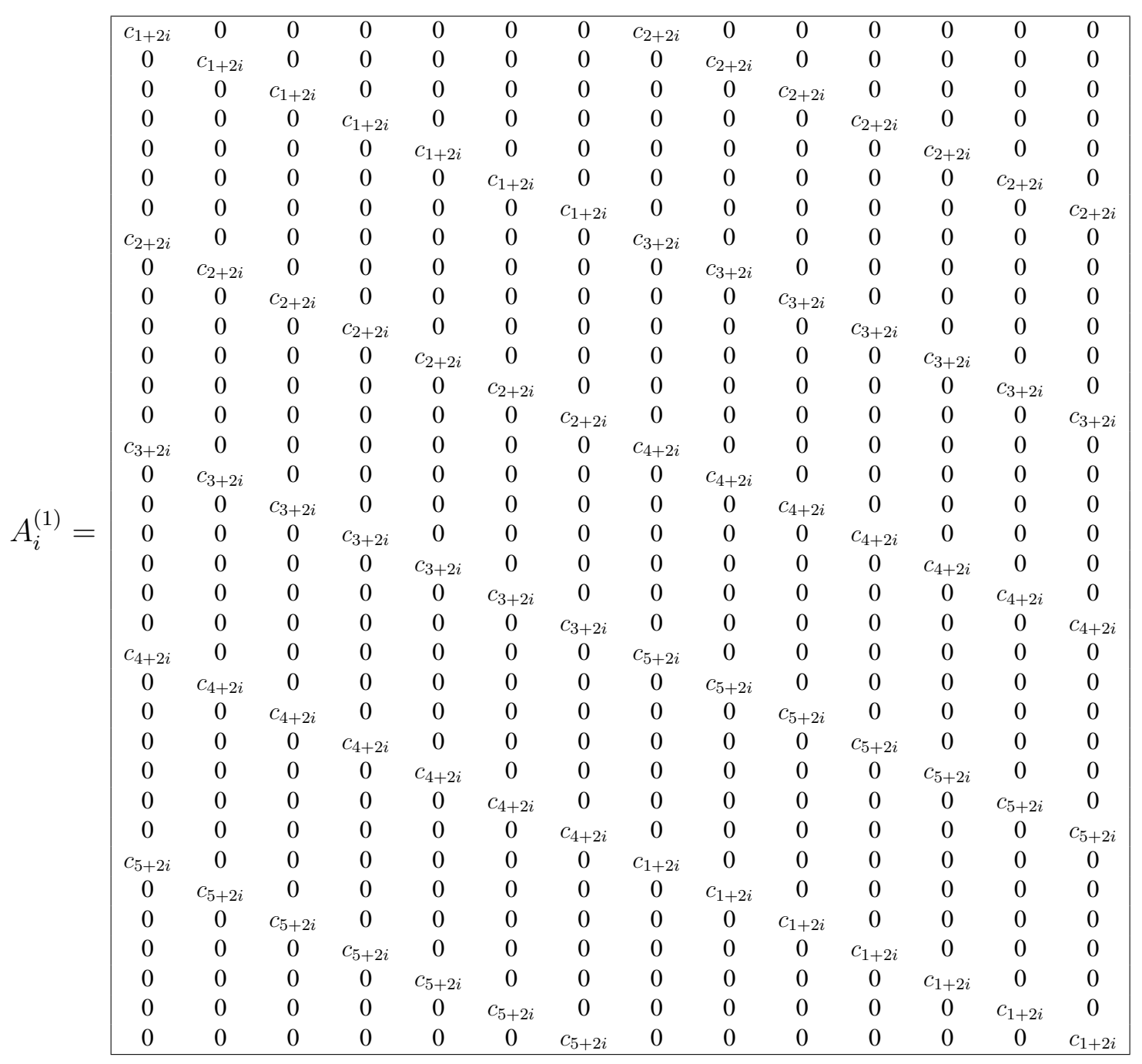

Figure 4.13: $35 \times 28$ colored matrix 


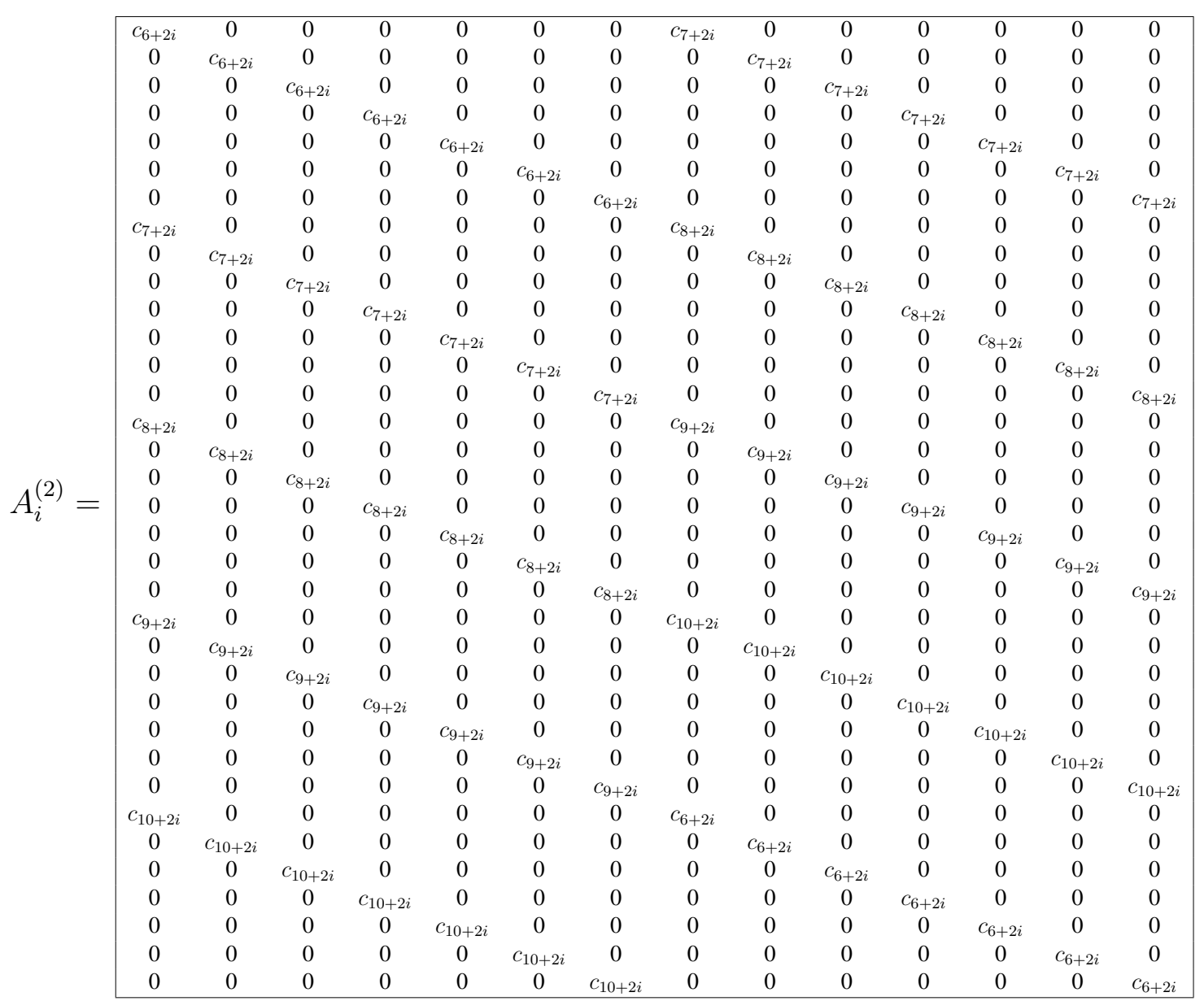

Figure 4.14: $35 \times 28$ colored matrix

Then the edge coloring incidence matrix of a $\operatorname{BIBD}(35,5,2)$ using 10 colors is given in Figure 4.15 .

Since $M_{i}$ is semi-properly colored, by Theorem 2.12 we can properly color a $\operatorname{BIBD}(35,5, \lambda)$.

Lemma 4.12. There exists a properly colored $\operatorname{BIBD}(16,5, \lambda)$ for $\lambda=4 k, k \in \mathbb{Z}^{+}, k \geq 3$.

Proof: Let $A$ be a $16 \times 16$ circulant matrix whose first row is [0100000000000000]. Then the edge coloring incidence matrix of $\operatorname{BIBD}(16,5,4)$ using 12 colors is given below. Let $B$ be the first 8 rows of a $16 \times 16$ circulant matrix whose first row is [0100000000000000]. Then the edge-coloring incidence matrix of a $\operatorname{BIBD}(16,5,4)$ using 12 colors is given in Figure 4.16

Since $M_{i}$ is semi-properly colored, by Theorem 2.12 we can properly color a $\operatorname{BIBD}(16,5, \lambda)$. 


\begin{tabular}{|c|c|c|c|}
\hline$\left(c_{1+2 i}\right) A^{23}$ & 0 & $\left(c_{2+2 i}\right) A^{3}$ & 0 \\
\hline$\left(c_{2+2 i}\right) I$ & 0 & $\left(c_{1+2 i}\right) A^{2}$ & 0 \\
\hline 0 & $\left(c_{3+2 i}\right) I$ & $\left(c_{4+2 i}\right) A^{3}$ & 0 \\
\hline$\left(c_{3+2 i}\right) A^{27}$ & $\left(c_{4+2 i}\right) A^{17}$ & 0 & 0 \\
\hline$\left(c_{4+2 i}\right) A^{27}$ & $\left(c_{5+2 i}\right) A^{13}$ & 0 & 0 \\
\hline$\left(c_{5+2 i}\right) A^{33}$ & 0 & $\left(c_{8+2 i}\right) A^{18}$ & 0 \\
\hline 0 & 0 & 0 & $A^{(1)}$ \\
\hline$\left(c_{7+2 i}\right) I$ & $\left(c_{8+2 i}\right) A^{8}$ & 0 & 0 \\
\hline 0 & $\left(c_{2+2 i}\right) A^{17}$ & $\left(c_{3+2 i}\right) A^{12}$ & 0 \\
\hline$\left(c_{10+2 i}\right) A^{33}$ & 0 & $\left(c_{7+2 i}\right) A^{12}$ & 0 \\
\hline$\left(c_{9+2 i}\right) A^{33}$ & $\left(c_{6+2 i}\right) A^{8}$ & 0 & 0 \\
\hline$\left(c_{6+2 i}\right) I$ & 0 & $\left(c_{5+2 i}\right) A^{12}$ & 0 \\
\hline$\left(c_{8+2 i}\right) I$ & $\left(c_{1+2 i}\right) A^{13}$ & 0 & 0 \\
\hline 0 & 0 & 0 & $A^{(2)}$ \\
\hline 0 & $\left(c_{9+2 i}\right) A^{32}$ & $\left(c_{10+2 i}\right) A^{18}$ & 0 \\
\hline 0 & $\left(c_{7+2 i}\right) A^{13}$ & $\left(c_{6+2 i}\right) A^{18}$ & 0 \\
\hline 0 & $\left(c_{10+2 i}\right) A^{17}$ & $\left(c_{9+2 i}\right) I$ & 0 \\
\hline
\end{tabular}

Figure 4.15: Edge-Coloring Incidence Matrix of a $\operatorname{BIBD}(35,5,2)$.

\begin{tabular}{|c|c|c|c|}
\hline \multirow{8}{*}{$M_{i}=$} & 0 & $\left(c_{1+4 i}\right) I$ & $\left(c_{2+4 i}\right) I+\left(c_{3+4 i}\right) A^{14}+\left(c_{4+4 i}\right) A^{15}$ \\
\hline & $\left(c_{1+4 i}\right) I$ & $\left(c_{2+4 i}\right) A^{8}$ & $\left(c_{7+4 i}\right) I+\left(c_{8+4 i}\right) A^{15}$ \\
\hline & $\left(c_{2+4 i}\right) A^{11}+\left(c_{3+4 i}\right) A^{14}$ & $\left(c_{4+4 i}\right) A^{11}$ & $\left(c_{1+4 i}\right) I$ \\
\hline & $\left(c_{4+4 i}\right) A^{4}+\left(c_{5+4 i}\right) A^{8}$ & $\left(c_{7+4 i}\right) A^{15}$ & $\left(c_{6+4 i}\right) H^{13}$ \\
\hline & $\left(c_{7+4 i}\right) I$ & $\left(c_{6+4 i}\right) I+\left(c_{8+4 i}\right) A^{11}$ & $\left(c_{5+4 i}\right) A^{14}$ \\
\hline & $\left(c_{6+4 i}\right) A^{4}+\left(c_{8+4 i}\right) A^{1} 4$ & $\left(c_{5+4 i}\right) A^{6}$ & $\left(c_{11+4 i}\right) A^{15}$ \\
\hline & $\left(c_{9+4 i}\right) A^{11}$ & $\left(c_{10+4 i}\right) A^{6}+\left(c_{11+4 i}\right) A^{15}$ & $\left(c_{12+4 i}\right) I$ \\
\hline & $\left(c_{10+4 i}\right) I+\left(c_{11+4 i}\right) B^{8}$ & $\left(c_{9+4 i}\right) I+\left(c_{12+4 i}\right) B^{8}$ & 0 \\
\hline
\end{tabular}

Figure 4.16: Edge-Coloring Incidence Matrix of a $\operatorname{BIBD}(16,5,4)$

Definition 4.13. A $g$-circulant matrix is an $n \times n$ square matrix of complex numbers, in which each row (except the first) is obtained from the preceding row by shifting the elements cyclically $g$ columns to the right.

Example 4.14. A 3-circulant matrix of order 7 whose first row is [1000000].

\begin{tabular}{|l|l|l|l|l|l|l|}
\hline 1 & 0 & 0 & 0 & 0 & 0 & 0 \\
\hline 0 & 0 & 0 & 1 & 0 & 0 & 0 \\
\hline 0 & 0 & 0 & 0 & 0 & 0 & 1 \\
\hline 0 & 0 & 1 & 0 & 0 & 0 & 0 \\
\hline 0 & 0 & 0 & 0 & 0 & 1 & 0 \\
\hline 0 & 1 & 0 & 0 & 0 & 0 & 0 \\
\hline 0 & 0 & 0 & 0 & 1 & 0 & 0 \\
\hline
\end{tabular}


Lemma 4.15. There exists a properly colored $\operatorname{BIBD}(50,5, \lambda)$ for $\lambda=4 k, k \in \mathbb{Z}^{+}, k \geq 3$.

Proof: Let $A$ be the $49 \times 49$ circulant matrix whose first row is

$$
\text { [0100000000000000000000000000000000000000000000000]. }
$$

Let $B_{1}$ be the $49 \times 4925$-circulant matrix whose first row is

$$
\text { [1000000000000000000000000000000000000000000000000]. }
$$

Let $B_{2}$ be the $49 \times 4937$-circulant matrix whose first row is

$$
\text { [1000000000000000000000000000000000000000000000000]. }
$$

Then the edge-coloring incidence matrix of a $\operatorname{BIBD}(50,5,4)$ using 12 colors is given in Figures 4.17 and 4.18 .

Since $M_{i}$ concatenated with $N_{i}$ is semi-properly colored, by Theorem 2.12 we can properly color a $\operatorname{BIBD}(50,5, \lambda)$.

Lemma 4.16. There exists a properly colored $\operatorname{BIBD}(60,5, \lambda)$ for $\lambda=4 k, k \in \mathbb{Z}^{+}, k \geq 3$.

Proof: Let $A$ be the $59 \times 59$ circulant matrix whose first row is [01000000000000000000000000000000000000000000000000000000000].

Let $B_{1}$ be the $59 \times 596$-circulant matrix whose first row is [10000000000000000000000000000000000000000000000000000000000].

Let $B_{2}$ be the $59 \times 5912-$ circulant matrix whose first row is [10000000000000000000000000000000000000000000000000000000000].

Let $B_{3}$ be the $59 \times 5915$-circulant matrix whose first row is [10000000000000000000000000000000000000000000000000000000000].

Let $B_{4}$ be the $59 \times 5924$-circulant matrix whose first row is [10000000000000000000000000000000000000000000000000000000000]. Let $B_{5}$ be the $59 \times 5930$-circulant matrix whose first row is [10000000000000000000000000000000000000000000000000000000000]. Let $B_{6}$ be the $59 \times 5937$-circulant matrix whose first row is [10000000000000000000000000000000000000000000000000000000000]. 


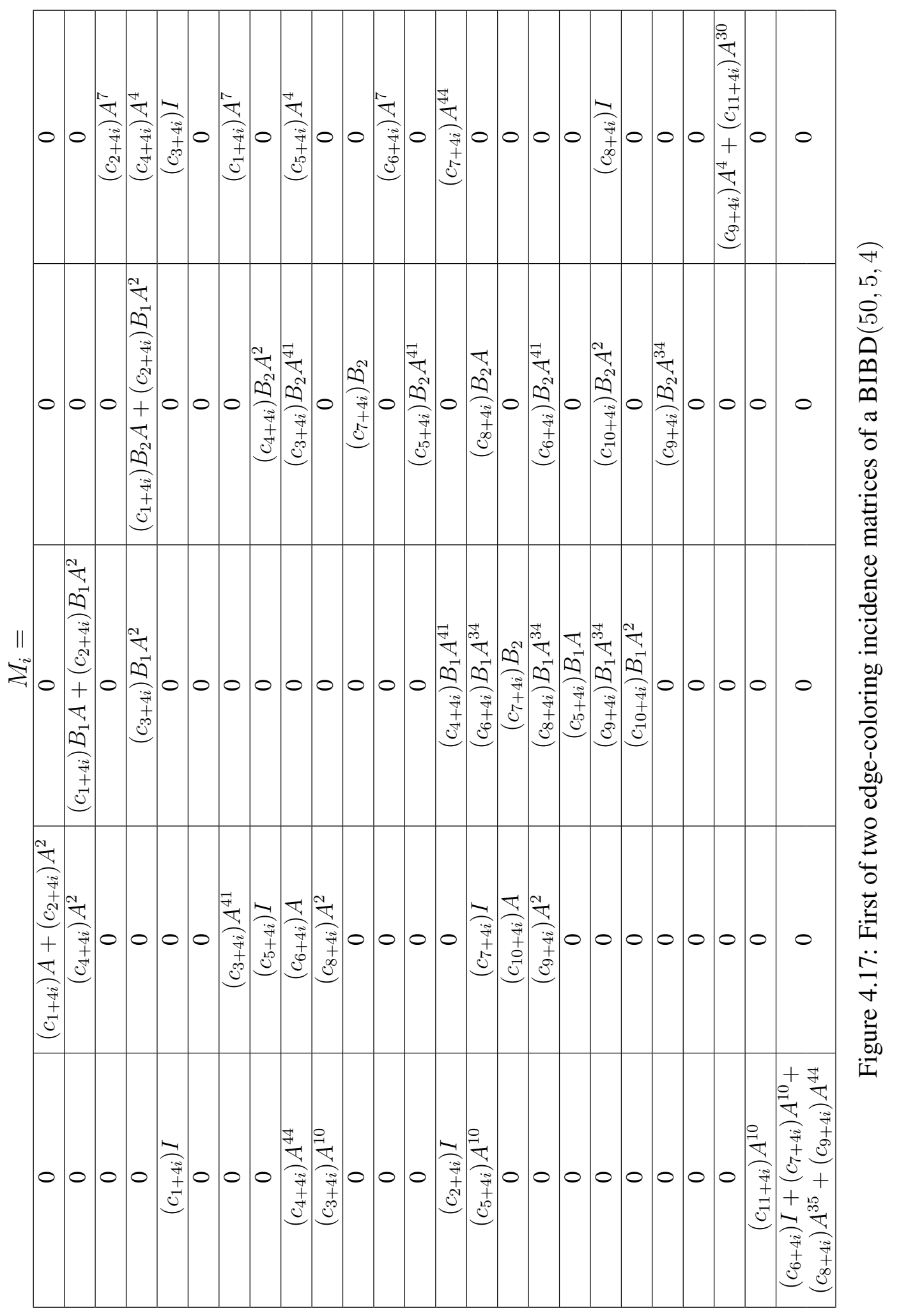




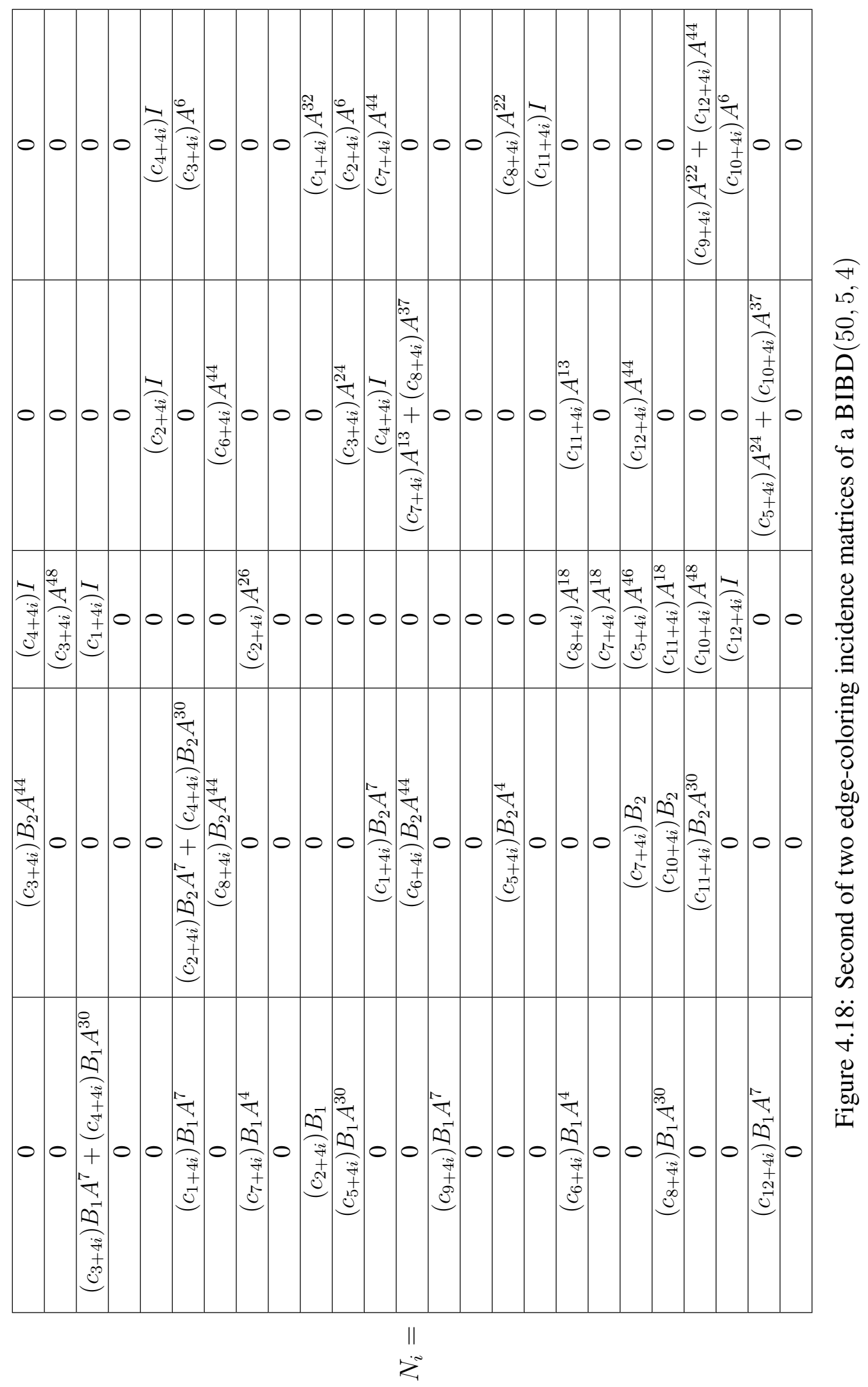


Let $B_{7}$ be the $59 \times 5948-$ circulant matrix whose first row is

$$
\text { [10000000000000000000000000000000000000000000000000000000000]. }
$$

Then the edge coloring incidence matrix of a $\operatorname{BIBD}(60,5,4)$ using 12 colors is given in Figures 4.19 and 4.20 .

Since $M_{i}$ concatenated with $N_{i}$ is semi-properly colored, by Theorem 2.12 we can properly color a $\operatorname{BIBD}(60,5, \lambda)$.

Because the process of checking each individual lemma for a correct direct construction is difficult, a program was built for the specific purpose of checking the small cases. Along with this is a csv (comma separated variables) and a tsv (tab separated variables) file of the edge-colored incidence matrix which are semi-properly colored. The program called bibdchecker.cpp is used to verify the design is a semi-properly colored BIBD. Mathematica was used to generate the properly colored versions of the BIBDs in csv and tsv form. Go to

http://www.mathlab.mtu.edu/ ms jukuri/Data.html

for the program and corresponding csv and tsv files.

\subsection{Main Results}

Lemma 4.17. If there exists $a 5-\operatorname{GDD}\left(a_{1}^{b_{1}} a_{2}^{b_{2}} \cdots a_{x}^{b_{x}}\right)$, and a properly colored $\operatorname{BIBD}\left(a_{i}, 5, \lambda\right)$ for all $i=1,2, \ldots, x$, then there exists a properly colored

$$
\operatorname{BIBD}\left(\sum_{i=1}^{x} a_{i} b_{i}, 5, \lambda\right) \text {. }
$$

Proof: Repeat each of the blocks in a $5-\operatorname{GDD}\left(a_{1}^{b_{1}} a_{2}^{b_{2}} \cdots a_{x}^{b_{x}}\right) \lambda$ times. For each block, we must color each edge a different color using the colors $c_{i} \in\left\{c_{1}, \ldots, c_{\lambda}\right\}$. Each corresponding edge in the $\lambda$ copies of the blocks must also be a different color. So we color the edges in the $\lambda$ copies of each block as follows. Form a $10 \times \lambda$ matrix. The rows of the matrix will be indexed by the 10 edges of $K_{5}$, and the columns will be indexed by the $\lambda$ copies of the block. The entries of the matrix will be the first 10 rows of an $\operatorname{LS}(\lambda)$. Now the only pairs that have not been covered are the pairs which lie within the groups. So we place a properly colored $\operatorname{BIBD}\left(a_{i}, 5, \lambda\right)$ on each group for all $i=1,2, \ldots, x$. This forms a properly colored BIBD $\left(\sum_{i=1}^{x} a_{i} b_{i}, 5, \lambda\right)$. 


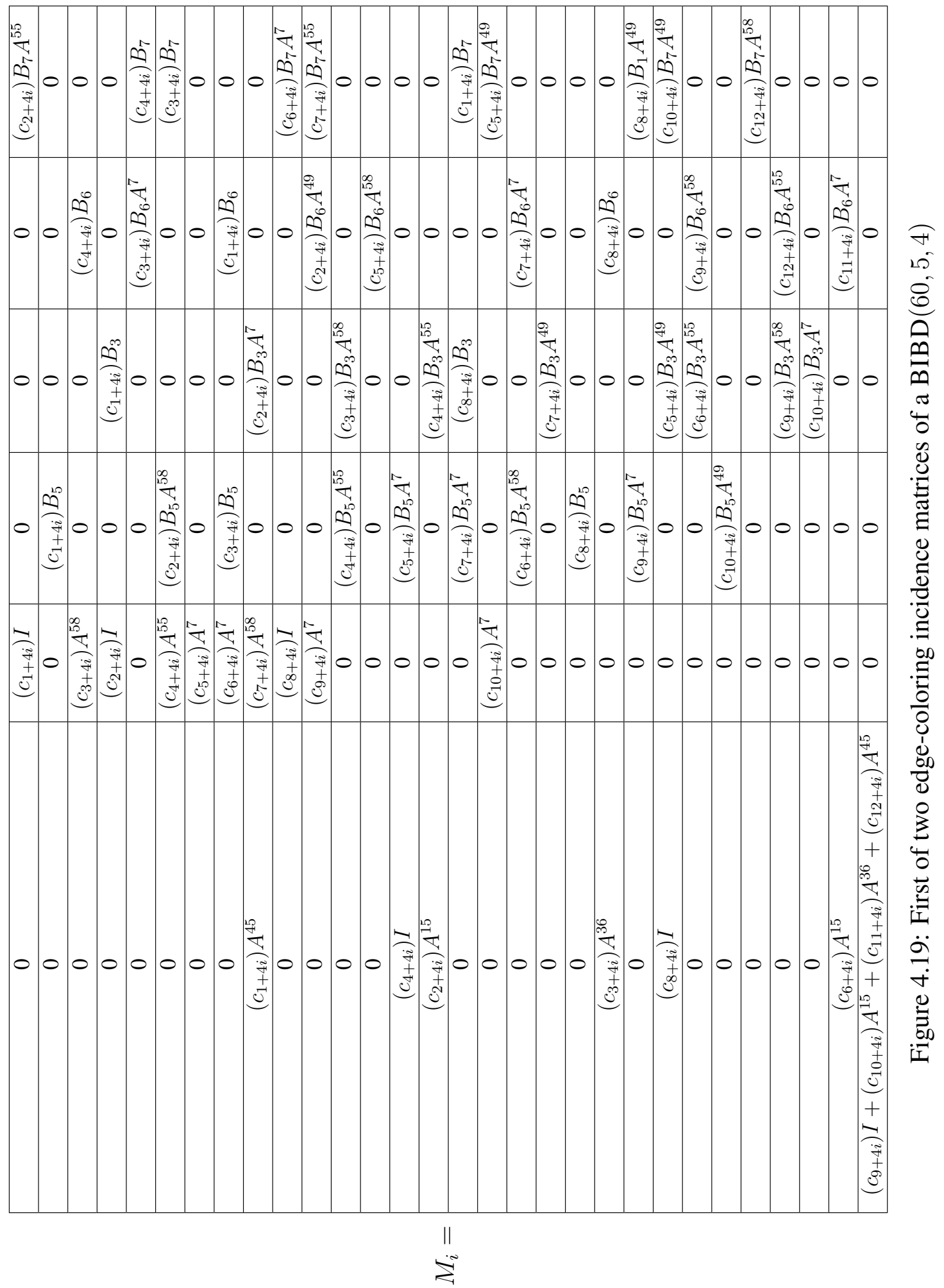




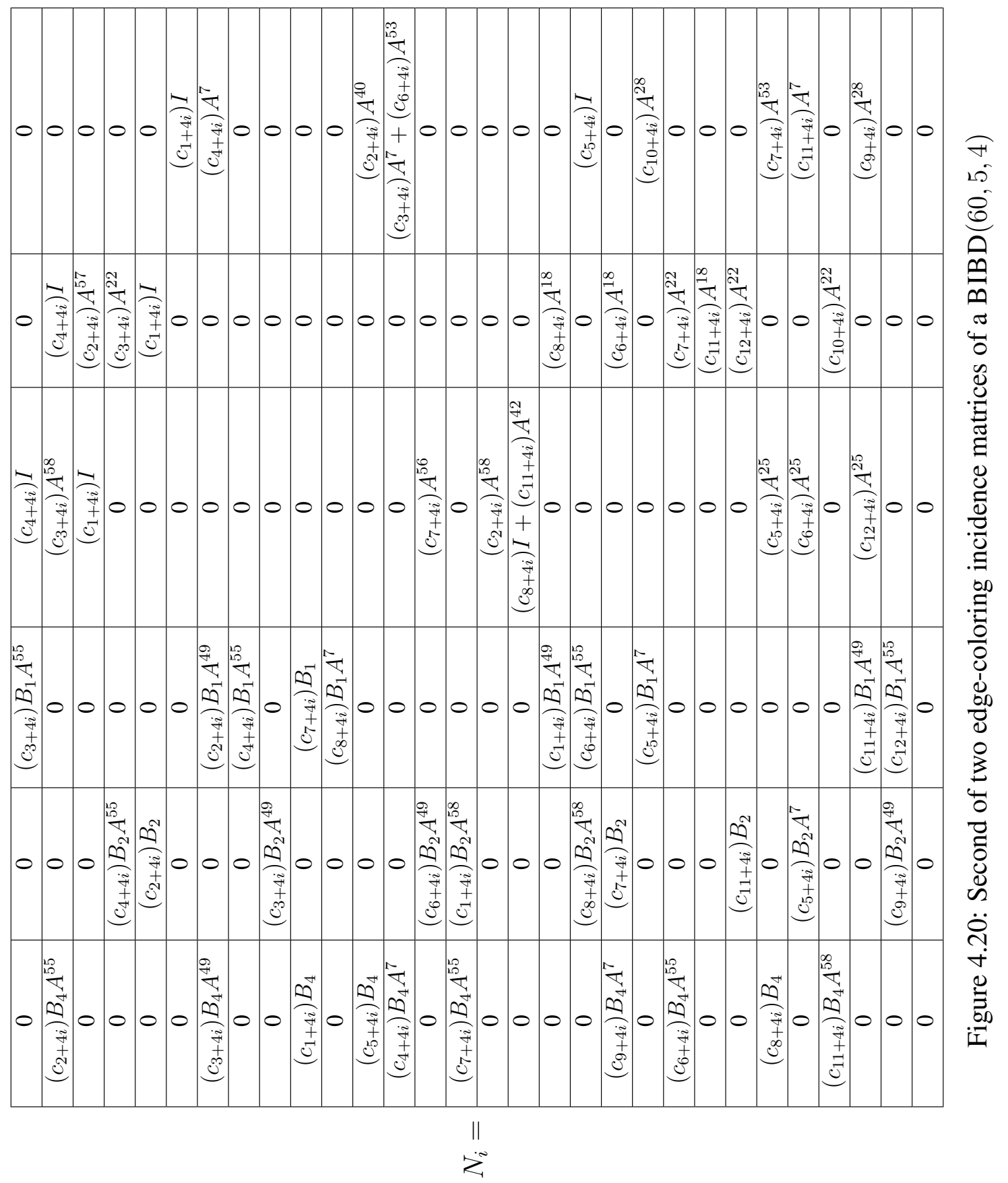


Lemma 4.18. If there exists a $5-\operatorname{GDD}\left(m^{u}\right)$ and a properly colored $\operatorname{BIBD}(m+1,5, \lambda)$, then there exists a properly colored $\operatorname{BIBD}(m u+1,5, \lambda)$.

Proof: Let $G_{i}$ for $i=1, \ldots, u$ be the $u$ groups of size $m$. Repeat each of the blocks in a $5-\operatorname{GDD}\left(m^{u}\right) \lambda$ times. For each block, we must color each edge a different color, using the colors $c_{i} \in\left\{c_{1}, \ldots, c_{\lambda}\right\}$. Each corresponding edge in the $\lambda$ copies of the block must also be a different color. So we color the edges in the $\lambda$ copies of each blocks as follows. Form a $10 \times \lambda$ matrix. The rows of the matrix will be indexed by the 10 edges of $K_{5}$, and the columns will be indexed by the $\lambda$ copies of the block. The entries of the matrix will be the first 10 rows of an $\operatorname{LS}(\lambda)$. Now the only pairs that have not been covered are the pairs which lie within the groups and pairs which contain the point $\{\infty\}$. So we place a properly colored $\operatorname{BIBD}(m+1,5, \lambda)$ on each $G_{i} \cup\{\infty\}$ for all $i=1,2, \ldots, u$. This forms a properly colored BIBD $(m u+1,5, \lambda)$.

Lemma 4.19. If there exists a $(5,2)-\operatorname{GDD}\left(m^{u}\right)$ and a properly colored $\operatorname{BIBD}(m, 5, \lambda)$, then there exists a properly colored $\operatorname{BIBD}(m u, 5, \lambda)$ where $\lambda=2 k, k \in \mathbb{Z}^{+}, k \geq 10$.

Proof: Repeat each of the blocks in a $(5,2)-\operatorname{GDD}\left(m^{u}\right) k$ times. For each block, we will color each edge a different color and each corresponding edge in the $k$ copies of the blocks must also be a different color. Form a $10 \times k$ matrix whose rows are indexed by the 10 edges of the $K_{5}$ and whose columns are indexed by the $k$ copies of the block. Because each edge is seen twice among all $10 \times k$ matrices which represent the blocks of the GDD, we assign color sets to each edge as follows. Let $C=\left\{c_{1}, c_{2}, \ldots, c_{k}\right\}$ and $D=\left\{d_{1}, d_{2}, \ldots, d_{k}\right\}$ be two distinct sets of $k$ colors each. For each edge in the block, if it is the first time it has occurred in a block of the GDD, we assign the color set $C$ to it, and if it is the second time it has occurred, we assign the color set $D$ to it. Let $t_{C}$ denote the number of edges which have color set $C$ assigned to it, and $t_{D}$ denote the number of edges which have color set $D$ assigned to it. The entries of our $10 \times k$ matrix will be the first $t_{C}$ rows of an $\operatorname{LS}(k)$ on the rows corresponding to the edges assigned with color set $C$ and the first $t_{D}$ rows of an $\mathrm{LS}(k)$ on the rows corresponding to the edges assigned with color set $D$. Consider some edge $e$. This edge, $e$, is colored with every color from $C$ exactly once in the $k$ copies of the first block of the GDD containing $e$, and it is colored with every color from $D$ exactly once in the $k$ copies of the second block in the GDD containing $e$. Furthermore, we can be sure that every edge in any block is colored with different colors because of the properties of Latin squares and because $C$ and $D$ are distinct.

The only pairs that have not been covered by the blocks of the GDD are pairs which lie within the groups. So we place a properly colored $\operatorname{BIBD}(m, 5, \lambda)$ on each group of the GDD. This forms a properly colored $\operatorname{BIBD}(m u, 5, \lambda)$.

Lemma 4.20. If there exists a $(5,2)-\operatorname{GDD}\left(m^{u}\right)$ and a properly colored $\operatorname{BIBD}(m+$ $1,5, \lambda)$, then there exists a properly colored $\operatorname{BIBD}(m u+1,5, \lambda)$ where $\lambda=2 k, k \in$ $\mathbb{Z}^{+}, k \geq 10$.

Proof: For $i=1,2, \ldots, u$ let $G_{i}$ denote the $i^{\text {th }}$ group of size $m$ in the GDD and let $\{\infty\}$ be a new point. Follow the same argument as in the first paragraph of the proof of Lemma 4.19. 
Then the only pairs that have not been covered by the blocks of the GDD are pairs which lie within the groups, and pairs which contain the point $\{\infty\}$. So place a properly colored $\operatorname{BIBD}(m+1,5, \lambda)$ on each $G_{i} \cup\{\infty\}$. This forms a properly colored $\operatorname{BIBD}(m u, 5, \lambda)$.

Lemma 4.21. Suppose there exists an $\operatorname{RBIBD}(v-m, 5,1)$ with t parallel classes. If there exists a properly colored $\mathrm{BIBD}(6,5, \lambda)$, and a properly colored $\operatorname{BIBD}(m, 5, \lambda)$ for $m \leq t$, then there exits a properly colored $\operatorname{BIBD}(v, 5, \lambda)$.

Proof: Let $\left\{\infty_{1}, \infty_{2}, \ldots, \infty_{m}\right\}$ be $m$ new points. Consider the parallel class $P_{i}$ for $i=$ $1, \ldots, m$. We take each block of the $P_{i}$ and join it with $\left\{\infty_{i}\right\}$. Now place a properly colored $\operatorname{BIBD}(6,5, \lambda)$ on each block of $P_{i} \cup\left\{\infty_{i}\right\}$. Now the only pairs that have not been covered are in the parallel classes $P_{i}$ for $i=m+1, \ldots, t$ and the pairs of the form $\left\{\left\{\infty_{i}, \infty_{j}\right\}: i, j \in\{1, \ldots, m\}\right\}$. So we place a properly colored $\operatorname{BIBD}(5,5, \lambda)$ on each block of the remaining parallel classes and we place a properly colored $\operatorname{BIBD}(m, 5, \lambda)$ on the set of points $\left\{\infty_{1}, \ldots, \infty_{m}\right\}$. This forms a properly colored $\operatorname{BIBD}(v, 5, \lambda)$ where $\lambda=4 k, \lambda \geq 3$.

Corollary 4.22. Let $m \leq 5 n+1$. If there exists properly colored $\operatorname{BIBD}(m, 5, \lambda)$, then there exists a properly colored $\operatorname{BIBD}(20 n+5+m, 5, \lambda)$ for each $\lambda=4 k, k \geq 3$, and $n \neq 2,11,17,23,32$.

Proof: There exists an $\operatorname{RBIBD}(20 n+5,5,1)$ for each $n \neq 2,11,17,23,32$ by Theorem 1.9 . This design has $5 n+1$ parallel classes. There exists a properly colored $\operatorname{BIBD}(6,5, \lambda)$ for $\lambda=4 k$ and $k \geq 3$. So apply Lemma 4.21 with $v=20 n+5$.

If the block size is five, then the possible $v$ values for all possible $\lambda$ values are as follows from (Hanani, [28]).

\begin{tabular}{rcrc}
\hline $\mathrm{k}$ & $\lambda$ & Conditions for $v$ & Exceptions \\
\hline 5 & 1 & $1,5(\bmod 20)$ & none \\
5 & 2 & $1,5(\bmod 10)$ & 15 \\
5 & 4 & $0,1(\bmod 5)$ & none \\
5 & 10 & $1(\bmod 2)$ & none \\
5 & 20 & all & none \\
\hline
\end{tabular}

Because we can properly color any $\lambda=10 k, k \in \mathbb{Z}^{+}, k \geq 1$ with Theorem 2.2 and any $\lambda$ when $v \equiv 1,5(\bmod 20)$ by Theorem 2.3, we will focus on $\lambda \equiv 0(\bmod 2)$ and $\lambda \equiv 0$ $(\bmod 4)$ in the following sections.

\subsection{1 $\lambda \equiv 0(\bmod 2)$}

In this section, we properly color all $\operatorname{BIBD}(v, 5, \lambda)$ s where $\lambda \equiv 0(\bmod 2)$ except possibly when $v \equiv 15,35,75,95(\bmod 100)$ and $\lambda=14,18$.

In this case, the necessary and sufficient conditions for the existence of a $\operatorname{BIBD}(v, 5, \lambda)$ are that $v \equiv 1,5(\bmod 10)$ except $v=15$ and $\lambda=2$. Note that when $v \equiv 1,5(\bmod 10)$ 
and $\lambda \equiv 0(\bmod 10)$ these are already covered by Theorem 2.2 , but the results in this section will also cover this case.

Theorem 4.23. There exists a proper coloring for every $\operatorname{BIBD}(v, 5, \lambda)$ for $\lambda=2 k, k \in$ $\mathbb{Z}^{+}, k \geq 5$, where $v \equiv 1,5(\bmod 10)$ except possibly when $\lambda=2 k, v \equiv 15,35,75,95$ $(\bmod 100)$ and $\lambda=14,18$.

Proof: Note that in each case we assume $\lambda=2 k, k \in \mathbb{Z}^{+}, k \geq 5$ unless otherwise stated. We first consider $v \equiv 5(\bmod 10)$. In other words $v \equiv 5$ or $15(\bmod 20)$.

Now suppose $v \equiv 1(\bmod 10)$. If $v \equiv 1(\bmod 20)$ then we can properly color a $\operatorname{BIBD}(v, 5, \lambda)$ by Theorem 2.3 . We deal with each subcase, $v \equiv 1(\bmod 20)$ and $v \equiv 11$ $(\bmod 20)$, separately.

Case 1: $\quad v \equiv 1(\bmod 20)$

By Theorem 2.3, we can properly color a $\operatorname{BIBD}(v, 5, \lambda)$ where $v \equiv 1,5(\bmod 20)$.

Case 2: $\quad v \equiv 11(\bmod 20)$

If $v \equiv 11(\bmod 20)$, then we have that either $v \equiv 11(\bmod 40)$ or $v \equiv 31(\bmod 40)$.

Case 2.1: $\quad v \equiv 11(\bmod 40)$

Let $v=11+40 x=1+10(1+4 x)$ for $x \geq 1$. There exists a $5-\operatorname{GDD}\left(10^{u}\right)$ where $u=1+4 x$ except possibly when $x=1,8$ by Theorem 1.7 . We can properly color a $\operatorname{BIBD}(11,5, \lambda)$ by Lemma 4.1. So we can apply Lemma 4.18 with $m=10, u=1+4 x$ and $v \neq 51,331$.

If $v=51$, then by Lemma 4.3 we can properly color a $\operatorname{BIBD}(51,5, \lambda)$. If $v=331=$ $1+30(11)$, then there exists a $5-\operatorname{GDD}\left(30^{11}\right)$ by Theorem 1.7 . There also exists a properly colored $\operatorname{BIBD}(31,5, \lambda)$ by Lemma 4.2 . Thus we can apply Lemma 4.18 to properly color a $\operatorname{BIBD}(331,5, \lambda)$.

Case 2.2: $\quad v \equiv 31(\bmod 40)$

If $v=31$, then we can properly colored a $\operatorname{BIBD}(31,5, \lambda)$ by Lemma 4.2 .

Let $v=31+40 x=1+10(3+4 x)$ for $x \geq 1$. There exists a $5-\operatorname{GDD}\left(10^{u}\right)$ where $u=3+4 x$ except possible when $x=1,3,5,6,8,9,11$ by Theorem 1.7 . We can properly color a $\operatorname{BIBD}(11,5, \lambda)$ by Lemma 4.1 . We can also properly color a $\operatorname{BIBD}(31,5, \lambda)$ by Lemma 4.2. So we can apply Lemma 4.18 with $m=10, u=3+4 x$ and $v \neq$ $71,151,231,271,351,391,471$.

If $v=71=5(14)+1$, then there exists a $5-\operatorname{GDD}\left(14^{5}\right)$ by Theorem 1.7 . There also exists a properly colored $\operatorname{BIBD}(15,5, \lambda)$ by Lemma 4.10 . Then by Lemma 4.18 , we can properly color a $\operatorname{BIBD}(71,5, \lambda)$. If $v=151=1+30(5)$, then there exists a $5-\operatorname{GDD}\left(30^{5}\right)$ by Theorem 1.7 . There also exists a properly colored $\operatorname{BIBD}(31,5, \lambda)$ by Lemma 4.2. So we can apply Lemma 4.18 to properly color a $\operatorname{BIBD}(151,5, \lambda)$. If $v=231=11(21)$, then there exists a $5-\operatorname{GDD}\left(11^{21}\right)$ by Theorem 1.7. There also exists a 
properly colored $\operatorname{BIBD}(11,5, \lambda)$ by Lemma 4.1. So we can apply Lemma 4.17 to properly color a $\operatorname{BIBD}(231,5, \lambda)$. If $v=271=1+54(5)$, then there exists a $5-\operatorname{GDD}\left(54^{5}\right)$ by Theorem 1.7. If $v=55$, then there exists a $5-\operatorname{GDD}\left(11^{5}\right)$ by Theorem 1.7. Since we can properly color a $\operatorname{BIBD}(11,5, \lambda)$ by Lemma 4.1, we can properly color a $\operatorname{BIBD}(55,5, \lambda)$ with Lemma 1.5. So we can apply Lemma 4.18 to properly color a $\operatorname{BIBD}(271,5, \lambda)$. If $v=351=70(5)+1$, then there exists a $5-\operatorname{GDD}\left(70^{5}\right)$ by Theorem 1.7 . There also exists a properly colored $\operatorname{BIBD}(71,5, \lambda)$ as stated above. So we can apply Lemma 4.18 to properly color a $\operatorname{BIBD}(351,5, \lambda)$. If $v=391=30(13)+1$ then there exists a $(5)-\operatorname{GDD}\left(30^{13}\right)$ by Theorem 1.7. There also exists a properly colored $\operatorname{BIBD}(31,5, \lambda)$ by Lemma 4.2. So we apply Lemma 4.18 to properly color a $\operatorname{BIBD}(391,5, \lambda)$. If $v=471$, then we can properly colored a $\operatorname{BIBD}(471,5, \lambda)$ by Lemma 2.10 .

Case 3: $v \equiv 15(\bmod 20)$

If $v \equiv 5(\bmod 20)$, then we properly color each $\operatorname{BIBD}(v, 5, \lambda)$ by Theorem 2.3. If $\lambda=10$, then by Theorem 2.2 we can properly color all $\operatorname{BIBD}(v, 5,10)$ where $v \equiv 15$ $(\bmod 20)$.

If $v=15$, then by Lemma 4.10 we can properly color all $\operatorname{BIBD}(15,5, \lambda)$. Let $v=$ $15+20 x=5(3+4 x)$ for $x \geq 1$. There exists a $(5,2)-\operatorname{GDD}\left(5^{u}\right)$ for $u=3+4 x$ by Theorem 1.8 . It is trivial to properly color a $\operatorname{BIBD}(5,5, \lambda)$. So we can apply Lemma 4.19 with $m=5, u=3+4 x$.

Now let $v=20 n+15$. If $n \geq 3$, then apply Corollary 4.22 with $m=10$ to properly color a $\operatorname{BIBD}(v, 5, \lambda)$ where $v \equiv 15(\bmod 20)$ and $\lambda=12,16$. This leaves the open cases of $v=15,35,55,235,355,475,655$ (corresponding to $n=0,1,2,11,17,23,32$ ). We construct the properly colored $\operatorname{BIBD}(15,5, \lambda)$ directly in Lemma 4.10. Also, we construct the properly colored $\operatorname{BIBD}(35,5, \lambda)$ directly in Lemma 4.11. For the other $v$, note that there exists an $\operatorname{RBIBD}(20(n-1)+5,5,1)$ with $5(n-1)+1=5 n+4$ parallel classes. In each case, $n \geq 10$, meaning we have at least 51 parallel classes. However, in each case we must only use $20 n+15-(20(n-1)+5)=30$ parallel classes. Thus we again apply Corollary 4.22 with $m=25$ and $n=n-1$.

To further break down the cases of $v \equiv 15(\bmod 20)$ we consider $v \equiv 55(\bmod 100)$. Let $v=55+100 x=(11+20 x) 5$ for $x \geq 0$. There exists a $5-\operatorname{GDD}\left((11+20 x)^{5}\right)$ by Theorem 1.7. There also exists a properly colored $\operatorname{BIBD}(11+20 x, 5, \lambda)$ by Case 2. Again, we can properly color a $\operatorname{BIBD}(5,5, \lambda)$. Thus, we can properly color a $\operatorname{BIBD}(v, 5, \lambda)$ for $v \equiv 55(\bmod 100)$.

Note that the remaining cases for a $\operatorname{BIBD}(v, 5, \lambda)$ where $v \equiv 15,35,75,95(\bmod 100)$ are $\lambda=14,18$.

Henceforth, there exists a proper coloring for every $\operatorname{BIBD}(v, 5, \lambda)$, where $v \equiv 1,5$ $(\bmod 10)$.

\subsection{2 $\lambda \equiv 0(\bmod 4)$}

In this section, we properly color all $\operatorname{BIBD}(v, 5, \lambda) \mathrm{s}$ where $\lambda \equiv 0(\bmod 4)$. Note the necessary and sufficient conditions for the existence of $\operatorname{B} \operatorname{BIBD}(v, 5, \lambda)$ are that $v \equiv 0,1$ 
$(\bmod 5)$. Note that when $v \equiv 0,1(\bmod 5)$ and $\lambda \equiv 0(\bmod 20)$ these are already covered by Theorem 2.2, but the results in this section satisfy this case as well.

Theorem 4.24. There exists a proper coloring for every $\operatorname{BIBD}(v, 5, \lambda)$ for $\lambda=4 k, k \geq 3$, where $v \equiv 0,1(\bmod 5)$.

Proof: Note that in each case we assume $\lambda=4 k, k \geq 3$ unless otherwise stated. We will break this problem up into two main cases, $v \equiv 0(\bmod 5)$ and $v \equiv 1(\bmod 5)$.

Case 1: $\quad v \equiv 0(\bmod 5)$

We consider four subcases: $v \equiv 0,5,10$, or $15(\bmod 20)$. If $v \equiv 0(\bmod 20)$, then we have that either $v \equiv 0(\bmod 40)$ or $v \equiv 20(\bmod 40)$.

Case 1.1: $\quad v \equiv 0(\bmod 40)$

If $v=40$, then by Lemma 4.8, we can properly color a $\operatorname{BIBD}(40,5, \lambda)$. If $v=$ $80=16(5)$, then there exists a $5-\operatorname{GDD}\left(16^{5}\right)$ by Theorem 1.7. We can properly color a $\operatorname{BIBD}(16,5, \lambda)$ by Lemma 4.12 . So we can properly color a $\operatorname{BIBD}(80,5, \lambda)$ by Lemma 4.17

Let $v=40 x=20(2 x)$ for $x \geq 3$. There exists a $5-\operatorname{GDD}\left(20^{2 x}\right)$ by Theorem 1.7 . We can properly color a $\operatorname{BIBD}(20,5, \lambda)$ by Lemma 4.6. So we can apply Lemma 4.17.

Case 1.2: $v \equiv 20(\bmod 40)$

If $v=20$, then by Lemma 4.6 we can properly color a $\operatorname{BIBD}(20,5, \lambda)$. If $v=60$, then by Lemma 4.16, we can properly color a $\operatorname{BIBD}(60,5, \lambda)$.

Let $v=20+40 x=20(1+2 x)$ for $x \geq 2$. There exists a $5-\operatorname{GDD}\left(20^{u}\right)$ for $u=1+2 x$ by Theorem 1.7. Because we can properly color a $\operatorname{BIBD}(20,5, \lambda)$, we can apply Lemma 4.17

Case 1.3: $\quad v \equiv 5(\bmod 20)$

By Theorem 2.3, we can properly color $\operatorname{B} \operatorname{BIBD}(v, 5, \lambda)$ where $v \equiv 1,5(\bmod 20)$.

Case 1.4: $v \equiv 10(\bmod 20)$

Let $v=20 n+10$. If $n \geq 1$, then apply Corollary 4.22 with $m=5$. This leaves the open cases of $v=50,230,350,470,650$ (corresponding to $n=0,2,11,17,23,32$ ). We construct the properly colored $\operatorname{BIBD}(10,5, \lambda)$ directly in Lemma 4.5 . We also construct the properly colored $\operatorname{BIBD}(50,5, \lambda)$ directly in Lemma 4.15. For the other $v$, note that these exists an $\operatorname{RBIBD}(20(n-1)+5,5,1)$ with $5(n-1)+1=5 n-4$ parallel classes. In each case, $n \geq 10$, meaning we have at least 51 parallel classes. However, in each case we must only use $20 n+10-(20(n-1)+5)=25$ parallel classes. Thus we again apply Corollary 4.22 with $m=25$ and $n=n-1$.

Case 1.5: $v \equiv 15(\bmod 20)$ 
Let $v=20 n+15$. If $n \geq 3$, then apply Corollary 4.22 with $m=10$. The open cases left are $v=15,35,55,235,355,475,655$ (corresponding to $n=0,1,2,11,17,23,32$ ). We construct the properly colored $\operatorname{BIBD}(15,5, \lambda)$ directly in Lemma 4.10 . We also construct the properly colored $\operatorname{BIBD}(35,5, \lambda)$ directly in Lemma 4.11. If $v=55$, there exists a $5-\operatorname{GDD}\left(11^{5}\right)$ by Theorem 1.7 . There also exists a properly colored $\operatorname{BIBD}(11,5, \lambda)$ by Theorem 4.23. So we apply Lemma 4.17 to properly color a $\operatorname{BIBD}(55,5, \lambda)$. For the other $v$, note that there exists an $\operatorname{RBIBD}(20(n-1)+5,5,1)$ with $5(n-1)+1=5 n-4$ parallel classes. In each case, $n \geq 10$, meaning we have at least 51 parallel classes. However, in each case we must only use $20 n+15-(20(n-1)+5)=30$ parallel classes. Thus we again apply Corollary 4.22 with $m=30$ and $n=n-1$.

Case 2: $\quad v \equiv 1(\bmod 5)$

If $v \equiv 1(\bmod 5)$, then $v \equiv 1,6,11$, or $16(\bmod 20)$. If $v \equiv 1(\bmod 20)$, we can properly color a $\operatorname{BIBD}(v, 5, \lambda)$ by Theorem 2.3. If $v \equiv 11(\bmod 20)$, then apply Theorem 4.23 to obtain a properly colored $\operatorname{BIBD}(v, 5, \lambda)$. This leaves two cases; $v \equiv 6,16$ $(\bmod 20)$.

Case 2.1: $v \equiv 6(\bmod 20)$

Let $v=6+20 x=1+5(1+4 x)$ for $x \geq 1$. There exists a $5-\operatorname{GDD}\left(5^{u}\right)$ for $u=1+4 x$ by Theorem 1.7. We can properly color a $\operatorname{BIBD}(6,5, \lambda)$ by Lemma 4.4. So we can apply Lemma 4.18 with $m=5$ and $u=1+4 x$.

Case 2.2: $\quad v \equiv 16(\bmod 20)$

Let $v=20 n+16$. If $v=16$, then by Lemma 4.12 we can properly color a $\operatorname{BIBD}(v, 5, \lambda)$. If $n \geq 3$, then apply Corollary 4.22 with $m=11$. This leaves the open cases of $v=$ $16,36,56,231,351,471,651$ (corresponding to $n=0,1,2,11,17,23,32$ ). We construct the properly colored $\operatorname{BIBD}(16,5, \lambda)$ directly in Lemma 4.12. If $v=36$, there exists a properly colored $\operatorname{BIBD}(36,5, \lambda)$ by Lemma 4.7. There also exists a properly colored $\operatorname{BIBD}(56,5, \lambda)$ by Lemma 4.9 . For the other $v$, note that there exists an $\operatorname{RBIBD}(20(n-$ $1)+5,5,1)$ either $(5, n-1)+1=5 n-4$ parallel classes. In each case, $n \geq 10$, meaning we have at least 51 parallel classes. However, in each case we must only use $20 n+16-(20(n-1)+5)=31$ parallel classes. Thus we again apply Corollary 4.22 with $m=31$ and $n=n-1$.

\subsection{Conclusion}

We are now in a position to prove the main theorem.

Theorem 4.25. There is a proper edge coloring for every $\operatorname{BIBD}(v, 5, \lambda)$ where $\lambda \geq 10$, except possibly when $\lambda=2 k, v \equiv 15,35,75,95(\bmod 100)$ and $\lambda=14,18$. 
Proof: Recall the necessary and sufficient conditions for the existence of a $\operatorname{BIBD}(v, 5, \lambda)$.

$$
\begin{aligned}
& \text { If } \lambda \equiv 1,3,5,7,9,11,13,15,17,19(\bmod 20) \text {, then } v \equiv 1,5(\bmod 20) \text {; } \\
& \text { If } \lambda \equiv 2,6,14,18(\bmod 20), \text { then } v \equiv 1,5(\bmod 10) ; \\
& \text { If } \lambda \equiv 4,8,12,16(\bmod 20) \text {, then } v \equiv 0,1(\bmod 5) ; \\
& \text { If } \lambda \equiv 10(\bmod 20) \text {, then } v \equiv 1(\bmod 2) ; \text { and } \\
& \text { If } \lambda \equiv 0(\bmod 20) \text {, then } v \geq 5 .
\end{aligned}
$$

If $\lambda \equiv 1,3,5,7,9,11,13,15,17,19(\bmod 20)$, then $v \equiv 1,5(\bmod 20)$ and we can properly color a $\operatorname{BIBD}(v, 5, \lambda)$ by applying Theorem 2.3. If $\lambda \equiv 10(\bmod 20)$ or $\lambda \equiv 0$ (mod 20), then $v \equiv 1(\bmod 2)$ or $v$ may be anything, respectfully, and we can properly color a $\operatorname{BIBD}(v, 5, \lambda)$ by applying Theorem 2.2. If $\lambda \equiv 2,6,14,18(\bmod 20)$, then $v \equiv$ $1,5(\bmod 10)$ and we apply Theorem 4.23 Finally, if $\lambda \equiv 4,8,12,16(\bmod 20)$, then $v \equiv 0,1,(\bmod 5)$ and we apply Theorem 4.24 . 


\section{Chapter 5}

\section{Alternate Method to Properly Color}

In this chapter, we take a graph theoretic approach to the proper coloring question in Chapters 3 and 4 . We also explain the strengths and weaknesses in application of the method in this chapter against the methods from Chapters 3 and 4 . The books by West [50] and Diestel [20] were used often as references in this chapter.

\subsection{Background in Graph Theory}

An $X, Y$-bigraph is a bipartite graph where the two partitions are called $X$ and $Y$. For the remainder of this chapter we adopt the notation $X$ and $Y$ as the partitioned sets of vertices from some arbitrary bipartite graph.

A saturation of $X$ is defined as a matching containing all points in $X$. We define a maximal matching as the largest possible matching set for a given graph $G$. If a matching $M$ saturates $X$, then for every $S \subseteq X$ there must be at least $|S|$ vertices in $Y$ that have neighbors (adjacent vertices) in $S$. Let $N_{G}(S)$ or $N(S)$ denote the set of neighboring or adjacent vertices to vertices in $S$. Clearly, $|N(S)| \geq|S|$ is a necessary condition. Phillip Hall proved that for all $S \subseteq X,|N(S)| \geq|S|$ is also a sufficient condition, hence why this condition is called Hall's Condition (Marriage Condition).

Theorem 5.1. (Marriage Theorem, [27]) An X, Y-bigraph $G$ has a matching that saturates $X$ if and only if $|N(S)| \geq|S|$ for all $S \subseteq X$.

Proof: We begin by showing if an $X, Y$-bigraph $G$ has a matching that saturates $X$, then $|N(S)| \geq|S|$ for all $S \subseteq X$. Consequently, if a matching saturates $X$, then for every $S \subseteq X$ there must be at least $|S|$ vertices in $Y$, and out of necessity $|N(S)| \geq|S|$.

Now we prove that Hall's Condition is sufficient using the contrapositive of the statement. We want to show if $M$ is a maximal matching in $G$ and $M$ does not saturate $X$, then we achieve a set $S \subseteq X$ such that $|N(S)|<|S|$. Let $u \in X$ be a vertex not in the matching $M$. Also let $S_{0}$ be the set of all vertices in $X$ reachable from $u$ by $M$-alternating paths in $G$ and $T_{0}$ be the set of all vertices in $Y$ reachable from $u$ by $M$-alternating paths in $G$. Note that $u \in S_{0}$ in Figure 5.1 . 


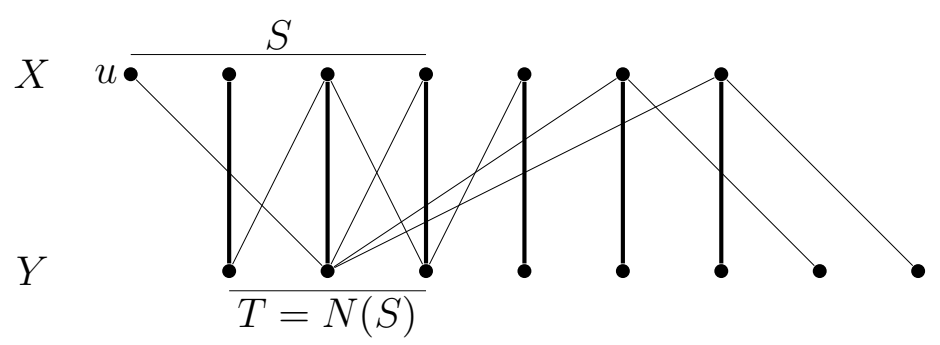

Figure 5.1: Proving the Marriage Theorem with alternating paths.

Our claim is that we must prove $M$ matches $T_{0}$ with $S_{0}-\{u\}$. Since the $M$-alternating paths from $u$ reach $Y$ through edges not in $M$ and return to $X$ along edges in $M$, to get to every vertex of $S_{0}-\{u\}$, one must pass through an edge in $M$ from a vertex in $T_{0}$. This shows there are no augmenting paths through $M$. Because there is no $M$-augmenting path, every vertex in $T_{0}$ is saturated. It follows that an $M$-alternating path reaching $y \in T_{0}$ may be extended via $M$ to a vertex of $S_{0}$. This means the edges in the matching $M$ yield a bijection from $T_{0}$ to $S_{0}-\{u\}$. Therefore we have $\left|T_{0}\right|=\left|S_{0}-\{u\}\right|$.

Because a matching between $T_{0}$ and $S_{0}-\{u\}$ exists, it tells us $T_{0} \subseteq N\left(S_{0}\right)$. Furthermore, because of the way we constructed $S_{0}$ and $T_{0}, T_{0}=N\left(S_{0}\right)$. Now suppose that $y \in Y \backslash T_{0}$ has an adjacent vertex $v \in S_{0}$. Since $y \notin T_{0}$, the edge $v y$ cannot be in $M$ since $u$ is unsaturated and the rest of $S_{0}$ is matched to $T_{0}$ by $M$. If we were to add $v y$ to an $M$ alternating path reaching $v$, then the $M$-alternating path would reach $Y$. This contradicts $y \notin T_{0}$. Therefore $v y$ cannot exist.

This shows $T_{0}=N\left(S_{0}\right)$, and thus $\left|N\left(S_{0}\right)\right|=|T|=\left|S_{0}\right|-1<\left|S_{0}\right|$ for our $S_{0}$. But since our $S_{0}$ is arbitrary, this is true for all $S$ and thus completes our proof.

Corollary 5.2. [50] For $k>0$, every $k$-regular bipartite graph has a perfect matching.

Proof: A perfect matching is a matching consisting of every vertex in the graph. Let $G$ be a $k$-regular $X, Y$-bigraph. Since each edge has two endpoints, we count the number of endpoints in $X$ and the number of endpoints in $Y$. Because for each edge one endpoint that lies in $X$ and there is an endpoint that in $Y$, the total number of endpoints are equivalent on both sides. It follows from the graph being $k$-regular that the number of endpoints in $X$ is $k|X|$ and the number of endpoints in $Y$ is $k|Y|$, so $k|X|=k|Y|$. Thus, $|X|=|Y|$. Thus a matching that saturates $X$ will also saturate $Y$ and this is matching is perfect. So we only need to prove there is a matching that saturates $X$. This is equivalent to verifying Hall's Condition.

Now we consider $S \subseteq X$. Let $m$ represent the number of edges from $S$ to $N(S)$. Because $G$ is a $k$-regular $X, Y$-bigraph, $m=k|S|$. The number of edges in $N(S)$ is $k|N(S)|$ and because there may be more edges coming into $N(S), k|S|=m \leq k|N(S)|$, and so $|N(S)| \geq|S|$. Finally, we note that we choose $S$ arbitrarily and thus we have established Hall's Condition.

To establish the understanding of the following theorem we need a little more terminol- 
ogy. A $k$-edge-coloring of $G$ is a labeling of the edges defined by the mapping $f: E \rightarrow S$, where $|S|=k$ and $E$ is the set of edges. These labels used will be colors. We call a $k$-edge-coloring proper if incident edges have different labels. This is equivalent to saying that each unique color generates a matching. An edge is incident to another edge if they share a common vertex. It is straightforward to call a graph $k$-edge-colorable if it has a proper $k$-edge-coloring. The edge-chromatic number, $\chi^{\prime}(G)$, of a simple $\operatorname{graph}^{1} G$ is given as a number $k$ such that $k$ is the minimum number where $G$ is $k$-edge-colorable. It follows that since edges sharing a vertex need different colors, $\chi^{\prime}(G) \geq \Delta(G)$ where $\Delta(G)$ is the maximum degree of all the vertices. It was proved independently by Vizing [48] and Gupta [26] that if $G$ is a simple graph, then $\Delta(G)+1$ colors suffice to color $G$.

Theorem 5.3. [50] If $H$ is bipartite, then $\chi^{\prime}(H)=\Delta(H)$.

Proof: The idea behind this proof is to establish the following. Given a $k$-regular bipartite graph $G$ and a subgraph $H$ of $G$, since $G$ has a perfect matching and yields a proper $\Delta(G)$ edge-coloring, we can achieve a proper $\Delta(G)$-edge-coloring on $H$ by removing edges. To prove this, though, we will start with a graph $H$ and show it generates $G$. First, Corollary 5.2 states every $k$-regular bipartite graph $G$ has a perfect matching. Hence, we can use induction on $G$ to show $\Delta(G)=k$ is the edge-chromatic number of $G$ and thus yields a proper $\Delta(G)$-edge-coloring. As a result, it suffices to show for every bipartite graph $H$ with maximum degree $k, H$ is a subgraph of a $k$-regular graph $G$, i.e. there is a $k$-regular bipartite graph $G$ containing $H$.

To construct $G$ from $H$, first consider the two partite sets of $H$ as $X$ and $Y$. We add vertices to the smaller set of $X$ and $Y$, if necessary, until the two sets are equal. If this new graph $H^{\prime}$ is not regular, then both $X$ and $Y$ have a vertex with degree less than $\Delta(G)$. Add an edge between these two vertices. Continue adding edges until $H^{\prime}$ becomes $k$-regular. Since $H^{\prime}$ is now $k$-regular, it has the same properties as $G$ and thus $H$ can yield a proper $k$-edge-coloring.

\subsection{Restating our Problem}

Consider the structure of an edge-incidence matrix of a $\operatorname{BIBD}(v, k, \lambda)$. We construct an $X, Y$-bigraph where $X$ is the set of all possible pairs of vertices (set of edges) and $Y$ is the set of blocks. There is an edge in this bipartite graph between $x \in X$ and $y \in Y$ if the pair represented by $x$ is in the block $y$. This will give us a bipartite graph with degree $\lambda$ on each $x$ and degree $\left(\begin{array}{l}k \\ 2\end{array}\right)$ on each $y$. Since $\lambda \geq\left(\begin{array}{c}k \\ 2\end{array}\right), \Delta(G)=\lambda$. Thus, by Theorem 5.3 $\lambda=\Delta(G)=\chi^{\prime}(G)$. Suppose that where there is an edge between a pair and a block, it means that edge in the block represents the $K_{k}$ of that block. Then we only need to properly color this bipartite graph in order to properly color the decompositions of $\lambda$ copies of $K_{v}$ into copies of $K_{k}$. Since we have constructed this bipartite graph from a $\operatorname{BIBD}(v, k, \lambda)$

\footnotetext{
${ }^{1}$ We say simple graph instead of loopless because the only types of graphs that we deal with are simple graphs.
} 
and we can properly color this graph, we can properly color any $\operatorname{BIBD}(v, k, \lambda)$. The result of this is given in the theorem below.

Theorem 5.4. There exists a properly colored decomposition of $\lambda$ copies of monochromatic $K_{v}$ into panchromatic $K_{k}$ if there exists a $\operatorname{BIBD}(v, k, \lambda)$.

\subsection{BIBD vs. PD}

Though this method solves our problem completely, the method also loses any structure that was given when using proper colorings of BIBDs. One such example is the correlation between BIBDs and another type of coloring problem associated with graph decompositions. A $k$-path decomposition ( $k$-PD) of a graph $\mathcal{H}$ is an edge-partition of $\mathcal{H}$ into subgraphs isomorphic to $P_{k+1}$, paths of length $k$. In [29], a question first posed by M. L. Yu is addressed. If $\mathcal{H}$ consists of $\lambda$ copies of $K_{v}$, and the edges of each copy of $K_{v}$ are monochromatically colored using $\lambda$ distinct colors, can one find a properly colored $k-\mathrm{PD}$ ? Again in [29], it was shown that if $v \geq 3$ is odd, then the necessary conditions are sufficient for the existence of a properly colored 2-PD with $\lambda=2$.

This idea does not extend directly to BIBDs with block size 4 because it is impossible to obtain panchromatic blocks when $\lambda<\left(\begin{array}{l}k \\ 2\end{array}\right)$. So we must construct equitably colored blocks. Let $|E|=e$ where $E$ is the set of edges in $K_{k}$ and $d=\left\lfloor\frac{e}{\lambda}\right\rfloor$. An equitably colored block is such that each color is used either $d$ or $d+1$ times to color the edges of the block. An equitable edge-coloring of a $\operatorname{BIBD}(v, k, \lambda)$ is then a decomposition of $\mathcal{H}$ into equitable blocks.

Before we revisit the idea of path decomposition, we define and remark upon equitable rectangles. Suppose $r, c$, and $v$ are positive integers. An equitable $(r, c ; v)$-rectangle is an $r \times c$ array $L$ where every entry is chosen from a $v$-set $X$, such that the following two properties are satisfied:

1. every symbol $x \in X$ occurs $\left\lfloor\frac{c}{v}\right\rfloor$ or $\left\lceil\frac{c}{v}\right\rceil$ times in each row of $L$.

2. every symbol $x \in X$ occurs $\left\lfloor\frac{r}{v}\right\rfloor$ or $\left\lceil\frac{r}{v}\right\rceil$ times in each column of $L$.

We will make use of the equitable rectangles studied in [14] and [45] as follows. If there exists a $\operatorname{BIBD}(v, k, 1)$, then we can equitably color the blocks of a $\operatorname{BIBD}(v, k, \lambda)$ in much the same way that we would color the design. Take $\lambda$ copies of each block in the $\operatorname{BIBD}(v, k, 1)$. For each block in the $\operatorname{BIBD}(v, k, 1)$ form a $\left(\begin{array}{l}k \\ 2\end{array}\right) \times \lambda$ matrix. The rows of the matrix will be indexed by the $\left(\begin{array}{l}k \\ 2\end{array}\right)$ pairs of points, and the columns will be indexed by the $\lambda$ copies of the block. The entries will be that of an equitable $\left(\left(\begin{array}{l}k \\ 2\end{array}\right), \lambda ; \lambda\right)$-rectangle. We suspect that one may be able to equitably color $\operatorname{BIBD}(v, k, \lambda)$ s with $2 \leq \lambda \leq\left(\begin{array}{l}k \\ 2\end{array}\right)$ with $k=3,4$, or 5 by coloring some small designs directly, and then applying recursive techniques involving GDDs and equitable $(r, c ; v)$-rectangles but this is still open.

This leads us to believe it may be possible to extend this idea to properly color $k$-PDs by using equitably colored $\operatorname{BIBD}(v, k+1, \lambda)$ s. For example, consider the following equitably colored $\operatorname{BIBD}(5,4,3)$. 


\begin{tabular}{c|ccccc} 
& $B_{1}$ & $B_{2}$ & $B_{3}$ & $B_{4}$ & $B_{5}$ \\
\hline$\{1,2\}$ & 0 & 0 & $c_{1}$ & $c_{2}$ & $c_{3}$ \\
$\{2,3\}$ & $c_{3}$ & 0 & 0 & $c_{1}$ & $c_{2}$ \\
$\{3,4\}$ & $c_{2}$ & $c_{3}$ & 0 & 0 & $c_{1}$ \\
$\{4,5\}$ & $c_{1}$ & $c_{2}$ & $c_{3}$ & 0 & 0 \\
$\{5,1\}$ & 0 & $c_{1}$ & $c_{2}$ & $c_{3}$ & 0 \\
\hline$\{1,3\}$ & 0 & $c_{1}$ & 0 & $c_{3}$ & $c_{2}$ \\
$\{2,4\}$ & $c_{2}$ & 0 & $c_{1}$ & 0 & $c_{3}$ \\
$\{3,5\}$ & $c_{3}$ & $c_{2}$ & 0 & $c_{1}$ & 0 \\
$\{4,1\}$ & 0 & $c_{3}$ & $c_{2}$ & 0 & $c_{1}$ \\
$\{5,2\}$ & $c_{1}$ & 0 & $c_{3}$ & $c_{2}$ & 0
\end{tabular}

Each block of the design consists of 2 edges that are colored by $c_{i}$, for $i=1,2,3$. Furthermore, the edges in each block can easily be decomposed into properly colored paths of length 3.

If we consider the case where $k=5$ we cannot create $k$-path decompositions because the length of the longest path without a cycle is four. Thus, we have to construct other path lengths to get any results. If we look at the edge-colored incidence matrix of a $\operatorname{BIBD}(11,5,2)$ in Figure 4.1, we see that we can construct a 2-PD if the index on the colors is evaluated $(\bmod 2)$. It is not necessary that our path decompositions be uniform but for a more rigid and useful structure we assume it is so. If we let path decompositions be non-uniform, it may be possible to construct two 4-PDs and one 2-PD. But again, this is not possible given the structure of the alternate method.

Finally, because we don't have any structure to the bipartite graph consisting of the pairs and blocks, we do not know the general construction of the coloring and therefore, do not generate a properly colored path decomposition. 


\section{Chapter 6}

\section{Mutually Orthogonal Equitable Latin Rectangles}

At this time we will abandon all conventions of notation developed in Chapters 2, 3, 4, and 5 to switch over to a completely new topic about Latin squares. In this chapter we investigate the maximum number of possible mutually orthogonal equitable Latin rectangles for all possible parameters. In essence, we will show that there are at least $3-\operatorname{MOELR}(a, b ; n)$ for all $a, b$, and $n$ except possibly when

$$
\begin{aligned}
& a=9 \text { and } n=3 s, \text { where } s \equiv 2,4,5,7(\bmod 9), s>9 ; \\
& a=18 \text { and } n=3 s, \text { where } s \equiv 8,10,(\bmod 18), s>18 \\
& a=36 \text { and } n=6 s, \text { where } s \equiv 5,7,11,13,17,19,23,25,29,31(\bmod 36), s>36 .
\end{aligned}
$$

and a finite number of cases. To do this we use a plethora of examples to establish a solid understanding of each construction used in the main theorem.

\subsection{Mutually Orthogonal Latin Squares}

A Latin square of side $n$ (or order $n$ ) is an $n \times n$ array in which each cell contains a single symbol from an $n$-set $S$, such that each symbol occurs exactly once in each row and exactly once in each column. We denote a Latin square of order $n$ as $\operatorname{LS}(n)$. As an example, here is a Latin square of side 8 on the symbols $0,1, \ldots, 7$.

$\begin{array}{llllllll}0 & 1 & 2 & 3 & 4 & 5 & 6 & 7 \\ 1 & 0 & 3 & 4 & 5 & 6 & 7 & 2 \\ 2 & 3 & 5 & 0 & 6 & 7 & 4 & 1 \\ 3 & 4 & 0 & 7 & 1 & 2 & 5 & 6 \\ 4 & 5 & 6 & 1 & 7 & 0 & 2 & 3 \\ 5 & 6 & 7 & 2 & 0 & 3 & 1 & 4 \\ 6 & 7 & 4 & 5 & 2 & 1 & 3 & 0 \\ 7 & 2 & 1 & 6 & 3 & 4 & 0 & 5\end{array}$

Figure 6.1: Latin square of side 8 
In 1779 Euler introduced the famous 36 officers problem. Say there are 36 officers, each having a rank and regiment.

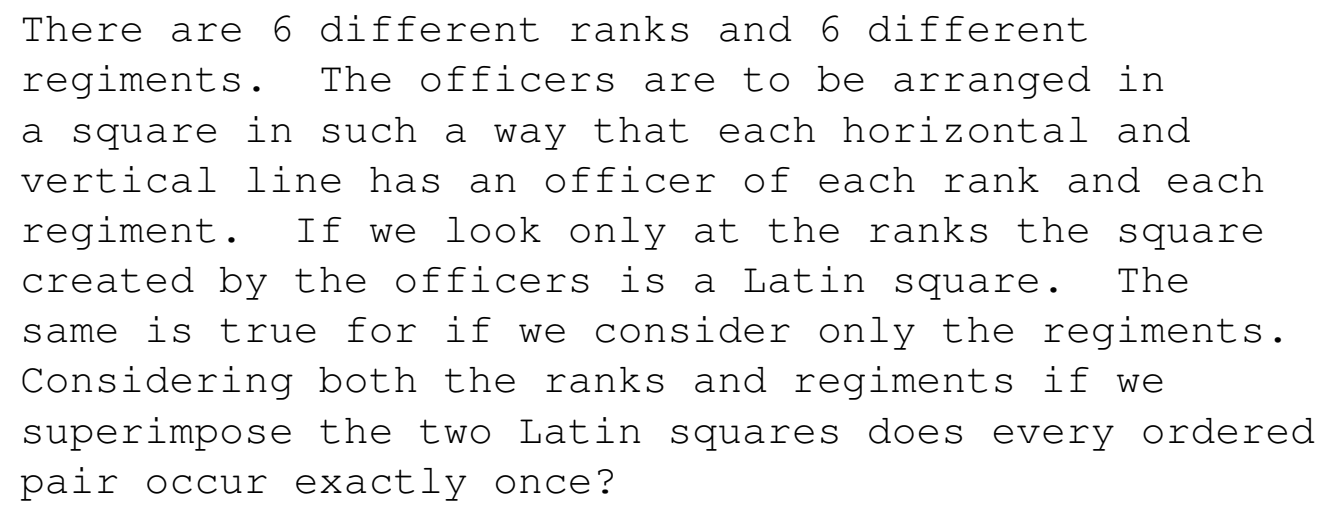

If two Latin squares have this property, then the squares are orthogonal. A set of $k$ Latin squares of order $n$, say $L_{1}, \ldots, L_{k}$, are said to be mutually orthogonal Latin squares if $L_{i}$ and $L_{j}$ are orthogonal for all $1 \leq i<j \leq k$. The maximum number of MOLS of order $n$ is denoted as $N_{\text {MOLS }}(n)$. Euler made a conjecture based on the knowledge that he knew $N_{\text {MOLS }}(2)=1$ and he strongly suspected $N_{\text {MOLS }}(6)=1$.

Conjecture 6.1. If $n \equiv 2(\bmod 4)$, then $N_{\text {MOLS }}(n)=1$.

Later, using BIBDs, Parker [41, 42] established a construction showing $N_{\text {MOLS }}(21) \geq$ 4. It was not until 1960 that Bose and Shrikhande [12] saw the work of Parker and made a shocking generalization using pairwise balanced designs rather than balanced incomplete block designs. A pairwise balanced design or a $\operatorname{PBD}(v, K, \lambda)$ is a $\mathrm{BIBD}$ with the size of the blocks being from the set $K$. As an example, Figure 6.2 is a $\operatorname{PBD}(10,\{3,4\})$ where the blocks are listed column-wise.

$\begin{array}{cccccccccccc}1 & 1 & 1 & 2 & 2 & 2 & 3 & 3 & 3 & 4 & 4 & 4 \\ 2 & 5 & 8 & 5 & 6 & 7 & 5 & 6 & 7 & 5 & 6 & 7 \\ 3 & 6 & 9 & 8 & 9 & 10 & 10 & 8 & 9 & 9 & 10 & 8 \\ 4 & 7 & 10 & & & & & & & & & \end{array}$

Figure 6.2: $\mathrm{A} \operatorname{PBD}(10,\{3,4\})$

Bose and Shrikhande proved $N_{\text {MOLS }}(22) \geq 2$ and $N_{\text {MOLS }}(66) \geq 5$. Later in [13], Parker joined with Bose and Shrikhande to show $N_{\text {MOLS }}(n) \geq 2$ for all $n \geq 10$.

In [52] Wilson developed a new class of constructions. And since then, the number of extensions and refinements of the techniques made by Bose-Shrikhande-Parker and the Wilson constructions are so plentiful that we can only mention where to refer for more literature on the subject. Colbourn and Dinitz in [18] give a more detailed description of constructions for small $n$. For a massive collection of results on the topics of combinatorial designs including this section and section 1.2, see [19]. 
Let us consider an example of three mutually orthogonal Latin squares of order 4, also denoted as 3-MOLS(4). The matrices $M_{1}, M_{2}, M_{3}$ below represent the three Latin squares of a $3-\operatorname{MOLS}(4)$ that are orthogonal.

$$
\left.\left.M_{1}=\begin{array}{llll}
1 & 2 & 3 & 4 \\
4 & 3 & 2 & 1 \\
2 & 1 & 4 & 3 \\
3 & 4 & 1 & 2
\end{array}\right] M_{2}=\begin{array}{llll}
1 & 2 & 3 & 4 \\
3 & 4 & 1 & 2 \\
4 & 3 & 2 & 1 \\
2 & 1 & 4 & 3
\end{array}\right] M_{3}=\begin{array}{llll}
1 & 2 & 3 & 4 \\
2 & 1 & 4 & 3 \\
3 & 4 & 1 & 2 \\
4 & 3 & 2 & 1
\end{array}
$$

The idea of Latin squares can be rewritten in such a way that we create a $(k+2) \times n^{2}$ array from $k-\operatorname{MOLS}(n)$. An orthogonal array $\mathrm{OA}(k+2, n)$ is a $(k+2) \times n^{2}$ array with entries from an $n$-set $S$ having the property that in any two rows, each pair of symbols from $S$ occurs exactly once. Figure 6.3 is an OA $(5,4)$ created from $3-\operatorname{MOLS}(4)$. The first row indicates the row of the Latin square. The second row indicates the column of the Latin square. The third, forth, and fifth rows are the elements in the first, second, and third Latin square respectively. So column five of Figure 6.3 would tell us in row two column one of the first, second, and third Latin squares the entries 4, 3, and 2 appear respectively.

\begin{tabular}{|llllllllllllllll|}
\hline 1 & 1 & 1 & 1 & 2 & 2 & 2 & 2 & 3 & 3 & 3 & 3 & 4 & 4 & 4 & 4 \\
1 & 2 & 3 & 4 & 1 & 2 & 3 & 4 & 1 & 2 & 3 & 4 & 1 & 2 & 3 & 4 \\
1 & 2 & 3 & 4 & 4 & 3 & 2 & 1 & 2 & 1 & 4 & 3 & 3 & 4 & 1 & 2 \\
1 & 2 & 3 & 4 & 3 & 4 & 1 & 2 & 4 & 3 & 2 & 1 & 2 & 1 & 4 & 3 \\
1 & 2 & 3 & 4 & 2 & 1 & 4 & 3 & 3 & 4 & 1 & 2 & 4 & 3 & 2 & 1 \\
\hline
\end{tabular}

Figure 6.3: An $\mathrm{OA}(5,4)$

In a similar way as we went from $3-\operatorname{MOLS}(4)$ to an $\mathrm{OA}(5,4)$ we may create a transversal design from an orthogonal array. A transversal design of order or group size $n$, block size $k$, and index $\lambda$, denoted $\operatorname{TD}_{\lambda}(k, n)$, is a triple $(V, \mathcal{G}, \mathcal{B})$, where

1. $\mathcal{V}$ is a set of $k n$ elements;

2. $\mathcal{G}$ is a partition of $\mathcal{V}$ into $k$ classes (the groups), each of size $n$;

3. $\mathcal{B}$ is a collection of $k$-subsets of $\mathcal{V}$ (the blocks);

4. every unordered pair of elements from $\mathcal{V}$ is contained either in exactly one group or in exactly $\lambda$ blocks, but not both.

When $\lambda=1$, one simply writes $\operatorname{TD}(k, n)$.

On the element set $\{1,2,3,4\} \times\{1,2,3,4,5\}$, the blocks of a $\operatorname{TD}(5,4)$ derived from an $\mathrm{OA}(5,4)$ are shown below. To go from the $\mathrm{OA}(5,4)$ to a $\mathrm{TD}(5,4)$ we let each column of the $\mathrm{OA}(5,4)$ be a block and list elements in the block as the element from the OA joined with the number denoting the row. 
$\{11,12,13,14,15\}$

$\{21,12,43,34,25\}$

$\{11,22,23,24,25\}$

$\{11,32,33,34,35\}$

$\{11,42,43,44,45\}$

$\{21,22,33,44,15\}$

$\{21,32,23,14,45\}$

$\{21,42,13,24,35\}$

$\{31,22,13,34,45\}$

$\{31,32,43,24,15\}$

$\{31,42,33,14,25\}$

$\{41,12,33,24,45\}$

$\{41,22,43,14,35\}$

$\{41,32,13,44,25\}$

$\{41,42,23,34,15\}$

\subsection{Background and Terms}

A $k \times n$ Latin rectangle is a $k \times n$ array (where $k \leq n$ ) in which each cell contains a single symbol from an $n$-set $S$, such that each symbol occurs exactly once in each row and at most once in each column. Two Latin rectangles are orthogonal if when superimposed no ordered pair of symbols appears more than once. A set of $m \times n$ Latin rectangles is mutually orthogonal, or a $\operatorname{MOLR}(m, n)$, if every two Latin rectangles in the set are orthogonal. The maximum number of $\operatorname{MOLR}(m, n)$ is denoted $N_{\text {MOLR }}(m, n)$.

Given positive integers $a, b$ and $n$, an equitable $(a, b ; n)$-rectangle is an $a \times b$ array, $L$, with entries from a $n$-set $S$, such that the following two properties are satisfied:

1. every symbol $s \in S$ occurs either $\left\lceil\frac{b}{n}\right\rceil$ or $\left\lfloor\frac{b}{n}\right\rfloor$; and times in each row of $L$.

2. every symbol $s \in S$ occurs either $\left\lceil\frac{a}{n}\right\rceil$ or $\left\lfloor\frac{a}{n}\right\rfloor$ times in each column of $L$.

An equitable $(a, b ; n)$-rectangle is row-regular if $n \mid b$, and it is column-regular if $n \mid a$. It is regular if it is both row- and column-regular. In a row-regular $(a, b ; n)$-rectangle, every symbol occurs exactly $\frac{b}{n}$ times in each row; in a column-regular $(a, b ; n)$-rectangle, every symbol occurs exactly $\frac{a}{n}$ times in each column.

Notice that an equitable $(a, b ; b)$-rectangle with $a \leq b$ is a Latin rectangle, and a Latin rectangle with $a=b$ is the same thing as a Latin square of side $a$.

Suppose that $L$ is an equitable $(a, b ; n)$-rectangle on symbol set $S$ and $R$ is an equitable $\left(a, b ; n^{\prime}\right)$-rectangle on symbol set $S^{\prime}$, where $a b=n n^{\prime}$. We say that $L$ and $R$ are orthogonal provided that, for every ordered pair $\left(s, s^{\prime}\right) \in S \times S^{\prime}$, there is a unique cell $C$ such that $L(C)=s$ and $R(C)=s^{\prime}$. (Equivalently, the superposition of $L$ and $R$ yields every ordered pair of symbols in $S \times S^{\prime}$.) It is easy to see that orthogonal equitable $(a, a ; a)$ rectangles are identical to orthogonal Latin squares of order $a$. Pairs of orthogonal equitable Latin rectangles were introduced in [45]. A complete solution for the existence of these rectangles was given in [14].

Now suppose that $L_{1}$ is an equitable $(a, b ; n)$-rectangle on symbol set $S, L_{2}$ is an equitable $\left(a, b ; n^{\prime}\right)$-rectangle on symbol set $S^{\prime}$, and $L_{3}$ is an equitable $\left(a, b ; n^{\prime \prime}\right)$-rectangle on symbol set $S^{\prime \prime}$ where $a b=n n^{\prime}=n^{\prime} n^{\prime \prime}=n n^{\prime \prime}$. Then it follows that $n=n^{\prime}=n^{\prime \prime}$. Therefore, a set of $k$ mutually orthogonal equitable Latin rectangles, or a $k-\operatorname{MOELR}(a, b ; n)$ is a set of $k$ pairwise equitable $(a, b ; n)$-rectangles on a symbol set $S$ where $a b=n^{2}$. We will denote the maximum number of $\operatorname{MoELR}(a, b ; n)$ by $N_{\text {MOelR }}(a, b ; n)$. For the remainder of the chapter we will say Latin rectangles instead of equitable Latin rectangles for simplicity.

To enhance the importance of mutually orthogonal equitable Latin rectangles, we will talk about mix functions and their applications. To start, we defined mix functions as 
Ristenpart and Rogaway did in [43] as follows. Let $|S|=r$. Suppose the mapping $f: S \times S \rightarrow S \times S$, and denote $f\left(s_{1}, s_{2}\right)=\left(f_{L}\left(s_{1}, s_{2}\right), f_{R}\left(s_{1}, s_{2}\right)\right)$ for all $s_{1}, s_{2} \in S$. A permutation is a rearrangement of the elements in an ordered set. Suppose that the following properties are satisfied:

1. $f(\cdot, \cdot)$ is a permutation of $S \times S$

2. if $s_{1} \in S$ is fixed, then $f_{L}\left(s_{1}, \cdot\right)$ is a permutation of $S$

3. if $s_{1} \in S$ is fixed, then $f_{R}\left(s_{1}, \cdot\right)$ is a permutation of $S$

4. if $s_{2} \in S$ is fixed, then $f_{L}\left(\cdot, s_{2}\right)$ is a permutation of $S$

5. if $s_{2} \in S$ is fixed, then $f_{R}\left(\cdot, s_{2}\right)$ is a permutation of $S$.

Then we say that $f$ is a MIX $(r)$ function.

In [43] it was observed that by using orthogonal Latin squares of order $r$ we can construct $\operatorname{MIX}(r)$ functions. Not only this, but the converse is also true.

Theorem 6.2. [45] Suppose that $|S|=r, f: S \times S \rightarrow S \times S$, and let L and $R$ be the $S \times S$ arrays defined by $L\left[s_{1}, s_{2}\right]=f_{L}\left(s_{1}, s_{2}\right)$ and $R\left[s_{1}, s_{2}\right]=f_{R}\left(s_{1}, s_{2}\right)$. Define two $r \times r$ arrays $L=\left(\lambda_{s_{1}, s_{2}}\right)$ and $R=\left(\rho_{s_{1}, s_{2}}\right)$ by the rules $\lambda_{s_{1}, s_{2}}=f_{L}\left(s_{1}, s_{2}\right)$ and $\rho_{s_{1}, s_{2}}=f_{R}\left(s_{1}, s_{2}\right)$ for all $s_{1}, s_{2}$. Then $f$ is a $\operatorname{MIX}(r)$ function if and only if $L$ and $R$ are orthogonal Latin squares of order $r$.

We now extend this definition of mix functions to generalized mix functions. Because mix functions have cryptographic applications from [43], generalized mix functions have potential applications in this field too. Which in turn may provide very useful information to allow mutually orthogonal equitable Latin rectangles to have cryptographic applications in the same manner.

In [14] a type of orthogonal equitable rectangle was studied called orthogonal generalized equitable rectangles (OGER). An OGER is defined as follows. Suppose $r, t, s_{1}, s_{2}$ are positive integers such that $r t=s_{1} s_{2}$. An OGER is a pair $(A, B)$ of two $r \times t$ rectangles satisfying the following properties:

1. $A=\left(a_{i, j}\right)$ is defined on a set $S_{1}$ of $s_{1}$ symbols and $B=\left(b_{i, j}\right)$ is defined on a set of $S_{2}$ of $s_{2}$ symbols, where $s_{1} s_{2}=r t$.

2. $A$ and $B$ are equitable on rows and equitable on columns.

3. $A$ and $B$ are orthogonal.

We denote $A$ and $B$ as $\left(r, t ; s_{1}, s_{2}\right)-$ OGER as in [14].

Example 6.3. $A(2,6 ; 3,4)-O G E R$. 


\begin{tabular}{|l|l|l|l|l|l|}
\hline 1 & 1 & 2 & 2 & 3 & 3 \\
\hline 2 & 2 & 3 & 3 & 1 & 1 \\
\hline
\end{tabular}

\begin{tabular}{|l|l|l|l|l|l|}
\hline 1 & 2 & 1 & 2 & 3 & 4 \\
\hline 3 & 4 & 2 & 1 & 4 & 3 \\
\hline
\end{tabular}

A striking difference between OGERs and MOELRs is that OGERs have symbol sets of different sizes defined on different arrays while MOELRs require a fixed symbol set among all of the arrays.

\subsection{Small Cases}

Example 6.4. $A$ 3-MOELR $(9,16 ; 12)$.

$M_{1}=$\begin{tabular}{cccccccccccccccc|}
1 & 3 & 4 & 2 & 1 & 3 & 4 & 2 & 7 & 9 & 10 & 8 & 7 & 9 & 10 & 8 \\
2 & 5 & 6 & 1 & 2 & 5 & 6 & 1 & 8 & 11 & 12 & 7 & 8 & 11 & 12 & 7 \\
3 & 6 & 1 & 4 & 3 & 6 & 1 & 4 & 9 & 12 & 7 & 10 & 9 & 12 & 7 & 10 \\
4 & 2 & 5 & 1 & 4 & 2 & 5 & 1 & 10 & 8 & 11 & 7 & 10 & 8 & 11 & 7 \\
5 & 1 & 3 & 6 & 5 & 1 & 3 & 6 & 11 & 7 & 9 & 12 & 11 & 7 & 9 & 12 \\
6 & 2 & 1 & 5 & 6 & 2 & 1 & 5 & 12 & 8 & 7 & 11 & 12 & 8 & 7 & 11 \\
3 & 4 & 2 & 6 & 3 & 4 & 2 & 6 & 9 & 10 & 8 & 12 & 9 & 10 & 8 & 12 \\
5 & 6 & 4 & 3 & 5 & 6 & 4 & 3 & 11 & 12 & 10 & 9 & 11 & 12 & 10 & 9 \\
2 & 4 & 3 & 5 & 2 & 4 & 3 & 5 & 8 & 10 & 9 & 11 & 8 & 10 & 9 & 11 \\
\hline
\end{tabular}

$M_{2}=$\begin{tabular}{cccccccccccccccc|}
1 & 2 & 5 & 3 & 7 & 8 & 11 & 9 & 1 & 2 & 5 & 3 & 7 & 8 & 11 & 9 \\
2 & 6 & 3 & 5 & 8 & 12 & 9 & 11 & 2 & 6 & 3 & 5 & 8 & 12 & 9 & 11 \\
3 & 1 & 4 & 6 & 9 & 7 & 10 & 12 & 3 & 1 & 4 & 6 & 9 & 7 & 10 & 12 \\
4 & 5 & 1 & 6 & 10 & 11 & 7 & 12 & 4 & 5 & 1 & 6 & 10 & 11 & 7 & 12 \\
5 & 3 & 6 & 4 & 11 & 9 & 12 & 10 & 5 & 3 & 6 & 4 & 11 & 9 & 12 & 10 \\
6 & 4 & 2 & 3 & 12 & 10 & 8 & 9 & 6 & 4 & 2 & 3 & 12 & 10 & 8 & 9 \\
4 & 1 & 6 & 2 & 10 & 7 & 12 & 8 & 4 & 1 & 6 & 2 & 10 & 7 & 12 & 8 \\
2 & 5 & 3 & 1 & 8 & 11 & 9 & 7 & 2 & 5 & 3 & 1 & 8 & 11 & 9 & 7 \\
1 & 2 & 5 & 4 & 7 & 8 & 11 & 10 & 1 & 2 & 5 & 4 & 7 & 8 & 11 & 10
\end{tabular}

$M_{3}=\begin{array}{cccccccccccccccc}1 & 4 & 3 & 6 & 7 & 10 & 9 & 12 & 7 & 10 & 9 & 12 & 1 & 4 & 3 & 6 \\ 2 & 3 & 5 & 4 & 8 & 9 & 11 & 10 & 8 & 9 & 11 & 10 & 2 & 3 & 5 & 4 \\ 3 & 4 & 6 & 2 & 9 & 10 & 12 & 8 & 9 & 10 & 12 & 8 & 3 & 4 & 6 & 2 \\ 4 & 1 & 2 & 5 & 10 & 7 & 8 & 11 & 10 & 7 & 8 & 11 & 4 & 1 & 2 & 5 \\ 5 & 2 & 1 & 3 & 11 & 8 & 7 & 9 & 11 & 8 & 7 & 9 & 5 & 2 & 1 & 3 \\ 6 & 5 & 3 & 4 & 12 & 11 & 9 & 10 & 12 & 11 & 9 & 10 & 6 & 5 & 3 & 4 \\ 2 & 6 & 4 & 1 & 8 & 12 & 10 & 7 & 8 & 12 & 10 & 7 & 2 & 6 & 4 & 1 \\ 6 & 2 & 1 & 5 & 12 & 8 & 7 & 11 & 12 & 8 & 7 & 11 & 6 & 2 & 1 & 5 \\ 3 & 5 & 6 & 1 & 9 & 11 & 12 & 7 & 9 & 11 & 12 & 7 & 3 & 5 & 6 & 1\end{array}$


It was known to Euler that a pair of orthogonal Latin squares of order 6 do not exist. However, Examples 6.5 and 6.6 show that there exists a $3-\operatorname{MOELR}(a, b ; 6)$ for $a=4, b=$ 9 and $a=3, b=12$. When $a=2$ and $b=18$, the existence of a $3-\operatorname{MOELR}(2,18 ; 6)$ is unresolved. See Lemma 6.27 for more on this.

Example 6.5. $A$ 3-MOELR $(4,9 ; 6)$.

$$
\begin{aligned}
& M_{1}=\begin{array}{lllllllll}
1 & 2 & 3 & 4 & 5 & 6 & 3 & 5 & 2 \\
3 & 5 & 6 & 2 & 1 & 2 & 4 & 6 & 4 \\
4 & 6 & 1 & 5 & 3 & 1 & 2 & 4 & 3 \\
2 & 1 & 4 & 1 & 6 & 5 & 6 & 3 & 5
\end{array} \\
& M_{2}=\begin{array}{lllllllll}
1 & 2 & 3 & 4 & 5 & 6 & 4 & 2 & 1 \\
2 & 6 & 1 & 5 & 3 & 4 & 1 & 5 & 2 \\
5 & 3 & 4 & 1 & 6 & 2 & 6 & 3 & 5 \\
3 & 5 & 6 & 6 & 4 & 3 & 2 & 1 & 4
\end{array} \\
& M_{3}=\begin{array}{lllllllll}
1 & 2 & 3 & 4 & 5 & 6 & 2 & 6 & 3 \\
4 & 3 & 4 & 1 & 2 & 5 & 6 & 2 & 5 \\
3 & 5 & 6 & 2 & 1 & 3 & 4 & 1 & 6 \\
6 & 4 & 2 & 5 & 3 & 4 & 1 & 5 & 1
\end{array}
\end{aligned}
$$

Example 6.6. $A$ 3-MOELR $(3,12 ; 6)$

$$
\begin{aligned}
& M_{1}=\begin{array}{llllllllllll|}
1 & 1 & 2 & 2 & 3 & 3 & 4 & 4 & 5 & 5 & 6 & 6 \\
2 & 2 & 1 & 1 & 4 & 4 & 3 & 3 & 6 & 6 & 5 & 5 \\
3 & 4 & 3 & 6 & 5 & 6 & 5 & 2 & 4 & 1 & 2 & 1
\end{array} \\
& M_{2}=\begin{array}{llllllllllll|}
1 & 2 & 1 & 2 & 4 & 6 & 2 & 4 & 3 & 5 & 5 & 6 \\
2 & 6 & 3 & 6 & 3 & 5 & 5 & 1 & 4 & 1 & 2 & 4 \\
3 & 1 & 2 & 2 & 6 & 3 & 1 & 5 & 6 & 4 & 4 & 5
\end{array} \\
& M_{3}=\begin{array}{llllllllllll|}
1 & 3 & 3 & 6 & 2 & 1 & 5 & 4 & 4 & 5 & 2 & 6 \\
2 & 4 & 2 & 5 & 1 & 3 & 6 & 5 & 3 & 4 & 6 & 1 \\
3 & 6 & 4 & 1 & 3 & 5 & 2 & 1 & 2 & 6 & 5 & 4 \\
\hline
\end{array}
\end{aligned}
$$

Let $\mathbb{F}_{n}$ be a finite field of order $n$. For each $f \in \mathbb{F}_{n}, f \neq 0$, define the $\mathbb{F}_{n} \times \mathbb{F}_{n}$ matrix $A_{f}$ by $A_{f}[x, y]=f x+y$ for all $x, y \in \mathbb{F}_{n}$. Then $\cup_{f} A_{f}$ forms a set of $\left|\mathbb{F}_{n}\right|-\operatorname{MOLS}(n)$. This construction is referred to as the finite field construction for MOLS (see [38]).

If $M$ is a Latin rectangle, we denote $M \backslash$ Row $i$ as the Latin rectangle obtained by removing Row $i$ from $M$. 
Example 6.7. A 3-MOELR $(4,25 ; 10)$.

Let $A_{1}, A_{2}, A_{3}$ be 3-MOLS(5) constructed using the finite field construction of sets of MOLS on the set of symbols $A=\left\{a_{1}, \cdots, a_{5}\right\}$. Let $B_{1}, B_{2}, B_{3}$ be a copy of this set of $3-\operatorname{MOLS}(5)$ on the set of symbols $B=\left\{b_{1}, \cdots, b_{5}\right\}$. Let

$$
\begin{aligned}
& M_{1}=\left[A_{1} \backslash \text { Row } 5, A_{2} \backslash \text { Row 5, } B_{1} \backslash \text { Row 5, } B_{2} \backslash \text { Row } 5, C_{1}\right] \\
& M_{2}=\left[A_{2} \backslash \text { Row 5, } B_{1} \backslash \text { Row 5, } A_{3} \backslash \text { Row } 5, B_{3} \backslash \text { Row } 5, C_{2}\right] \\
& M_{3}=\left[B_{3} \backslash \text { Row 5, } A_{3} \backslash \text { Row 5, } A_{2} \backslash \text { Row } 5, B_{1} \backslash \text { Row } 5, C_{3}\right]
\end{aligned}
$$

where

$$
\begin{gathered}
C_{1}=\left[\begin{array}{lll}
\text { Row } 5 & \text { of } & A_{1} \\
\text { Row } 5 & \text { of } & A_{2} \\
\text { Row } 5 & \text { of } & B_{1} \\
\text { Row } 5 & \text { of } & B_{2}
\end{array}\right], C_{2}=\left[\begin{array}{lll}
\text { Row } 5 & \text { of } & A_{2} \\
\text { Row } 5 & \text { of } & B_{1} \\
\text { Row } 5 & \text { of } & A_{3} \\
\text { Row } 5 & \text { of } & B_{3}
\end{array}\right], \\
C_{3}=\left[\begin{array}{lll}
\text { Row } 5 & \text { of } & B_{3} \\
\text { Row } 5 & \text { of } & A_{3} \\
\text { Row } 5 & \text { of } & A_{2} \\
\text { Row } 5 & \text { of } & B_{1}
\end{array}\right] .
\end{gathered}
$$

Then $M_{1}, M_{2}, M_{3}$ form a $3-\operatorname{MOELR}(4,25 ; 10)$. For each pair of rectangles $M_{i}, M_{j}$ for $i, j, \in\{1,2,3\}$, we have the property that every ordered pair of each of the following types occurs exactly once: $\left(a_{i}, a_{j}\right),\left(a_{i}, b_{j}\right),\left(b_{i}, b_{j}\right),\left(b_{i}, a_{j}\right)$ for all $i, j \in\{1, \cdots, 5\}$. Therefore, orthogonality holds. Consider any rectangle $M_{i}$ for $i \in\{1,2,3\}$. In any row of this rectangle, either the symbols from set $A$ each occur 2 times and the symbols from the set $B$ occur 3 times, or vice versa. Furthermore, because we used the finite field construction to construct our set of $\operatorname{MOLS}(5)$, we have that the pair $(k, k)$ for $k=0,1,2,3,4$ has appeared in row 1 for each pair of squares. Therefore, no symbol will be repeated in any column of any of the $C_{i}$ for $i \in\{1,2,3\}$.

We give a $3-\operatorname{MOELR}(4,25 ; 10)$ in Figure 6.4 .

Example 6.8. A 3-MOELR $(4,49 ; 14)$.

Let $A_{1}, A_{2}, A_{3}$ be 3-MOLS(7) constructed using the finite field construction of sets of MOLS on the set of symbols $A=\left\{a_{1}, \cdots, a_{7}\right\}$. Let $B_{1}, B_{2}, B_{3}$ be a copy of this set of 3 $\operatorname{MOLS}(7)$ on the set of symbols $B=\left\{b_{1}, \cdots, b_{7}\right\}$. Let

$$
\begin{aligned}
& M_{1}=\left[A_{1} \backslash \text { Rows 5,6,7, } A_{2} \backslash \text { Rows 5,6,7, } B_{1} \backslash \text { Row 5,6,7, } B_{2} \backslash \text { Rows 5,6,7, } C_{1}\right] \\
& M_{2}=\left[A_{2} \backslash \text { Rows 5,6,7, } B_{1} \backslash \text { Rows 5,6,7, } A_{3} \backslash \text { Rows 5,6,7, } B_{3} \backslash \text { Rows 5,6,7, } C_{2}\right] \\
& M_{3}=\left[B_{3} \backslash \text { Rows 5,6,7, } A_{3} \backslash \text { Rows 5,6,7, } A_{2} \backslash \text { Rows 5,6,7, } B_{1} \backslash \text { Rows 5,6,7, } C_{3}\right]
\end{aligned}
$$

where 


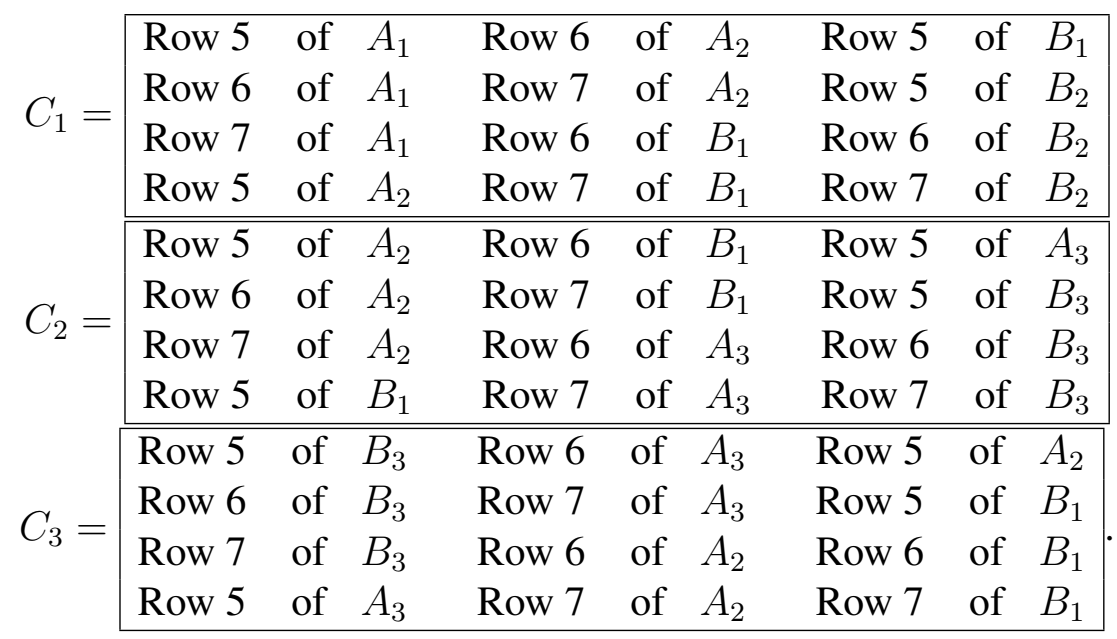

Then $M_{1}, M_{2}, M_{3}$ form a $3-\operatorname{MOELR}(4,49 ; 14)$. For each pair of rectangles $M_{i}, M_{j}$ for $i, j, \in\{1,2,3\}$, we have the property that every ordered pair of each of the following types occurs exactly once: $\left(a_{i}, a_{j}\right),\left(a_{i}, b_{j}\right),\left(b_{i}, b_{j}\right),\left(b_{i}, a_{j}\right)$ for all $i, j \in\{1, \cdots, 7\}$. Therefore, orthogonality holds. Consider any rectangle $M_{i}$ for $i \in\{1,2,3\}$. In any row of this rectangle, either the symbols from set $A$ each occur 4 times and the symbols from the set $B$ occur 3 times, or vice versa. Furthermore, because we used the finite field construction to construct our set of 3-MOLS (7), we can be sure that no symbol is repeated in any column of any of the $C_{i}$ for $i \in\{1,2,3\}$. This is because $2 \cdot 4 \neq 4,5$ or $6(\bmod 7)$, so columns $1-7$ of $C_{1}$ contain no repeated symbols. For columns 8-14 of $C_{2}$ there are no repeated symbols because $2 \cdot 5 \not \equiv 3 \cdot 5$ or $3 \cdot 6(\bmod 7)$ and $2 \cdot \not \equiv 3 \cdot 5$ or $3 \cdot 6(\bmod 7)$. Similarly, $4 \not \equiv 2 \cdot 4$ or $2 \cdot 5$ or $2 \cdot 6(\bmod 7)$, so columns $15-21$ of $C_{1}$ contain no repeated symbols. It is easy to see that no symbol is repeated in any of the other columns of $M_{i}$.

We give a $3-\operatorname{MOELR}(4,49 ; 14)$ in Figure 6.5 .

\begin{tabular}{|c|c|c|c|c|c|c|c|c|c|c|c|c|c|c|c|c|c|c|c|c|c|c|c|c|}
\hline \multirow{4}{*}{$M_{1}=$} & $\begin{array}{ll}0 & 1\end{array}$ & 2 & 3 & 4 & 0 & 1 & 2 & 3 & 4 & 5 & 6 & 7 & 8 & & 5 & 6 & 7 & 8 & 9 & 4 & 0 & 1 & 2 & \\
\hline & 12 & 3 & 4 & 0 & 2 & 3 & 4 & 0 & 1 & 6 & 7 & 8 & 9 & 5 & 7 & 8 & 9 & 5 & 6 & 3 & 4 & 0 & 1 & \\
\hline & 23 & 4 & 0 & 1 & 4 & 0 & 1 & 2 & 3 & 7 & 8 & 9 & 5 & 6 & 9 & 5 & 6 & 7 & 8 & 9 & 5 & 6 & 7 & 8 \\
\hline & 34 & 0 & 1 & 2 & 1 & 2 & 3 & 4 & 0 & 8 & 9 & 5 & 6 & 7 & 6 & 7 & 8 & 9 & 5 & 8 & 9 & 5 & 6 & \\
\hline \multirow{4}{*}{$M_{2}=$} & $\begin{array}{ll}0 & 1\end{array}$ & 2 & 3 & 4 & 5 & 6 & 7 & 8 & 9 & 0 & 1 & 2 & 3 & 4 & 5 & 6 & 7 & 8 & 9 & 3 & 4 & 5 & 6 & \\
\hline & 23 & 4 & 0 & 1 & 6 & 7 & 8 & 9 & 5 & 3 & 4 & 0 & 1 & 2 & 8 & 9 & 5 & 6 & 7 & 9 & 5 & 6 & 7 & 8 \\
\hline & 40 & 1 & 2 & 3 & 7 & 8 & 9 & 5 & 6 & 1 & 2 & 3 & 4 & 0 & 6 & 7 & 8 & 9 & 5 & 2 & 3 & 4 & 0 & 1 \\
\hline & 12 & 3 & 4 & 0 & 8 & 9 & 5 & 6 & 7 & 4 & 0 & 1 & 2 & 3 & 9 & 5 & 6 & 7 & 8 & 7 & 8 & 9 & 5 & 6 \\
\hline \multirow{4}{*}{$M_{3}=$} & 56 & 7 & 8 & 9 & 0 & 1 & 2 & 3 & 4 & 0 & 1 & 2 & 3 & 4 & 5 & 6 & 7 & 8 & 9 & 7 & 8 & 9 & 6 & \\
\hline & $\begin{array}{ll}89 \\
\end{array}$ & 5 & 6 & 7 & 3 & 4 & 0 & 1 & 2 & 2 & 3 & 4 & 0 & 1 & 6 & 7 & 8 & 9 & 5 & 2 & 3 & 4 & 0 & \\
\hline & $\begin{array}{ll}67 \\
\end{array}$ & 8 & 9 & 5 & 1 & 2 & 3 & 4 & 0 & 4 & 0 & 1 & 2 & 3 & 7 & 8 & 9 & 5 & 6 & 3 & 4 & 0 & 1 & \\
\hline & 9 & 6 & 7 & 8 & 4 & 0 & 1 & 2 & 3 & 1 & 2 & 3 & 4 & 0 & 8 & 9 & 5 & 6 & 7 & 9 & 5 & 6 & 7 & \\
\hline
\end{tabular}

Figure 6.4: A 3-MOELR $(4,25 ; 10)$ 


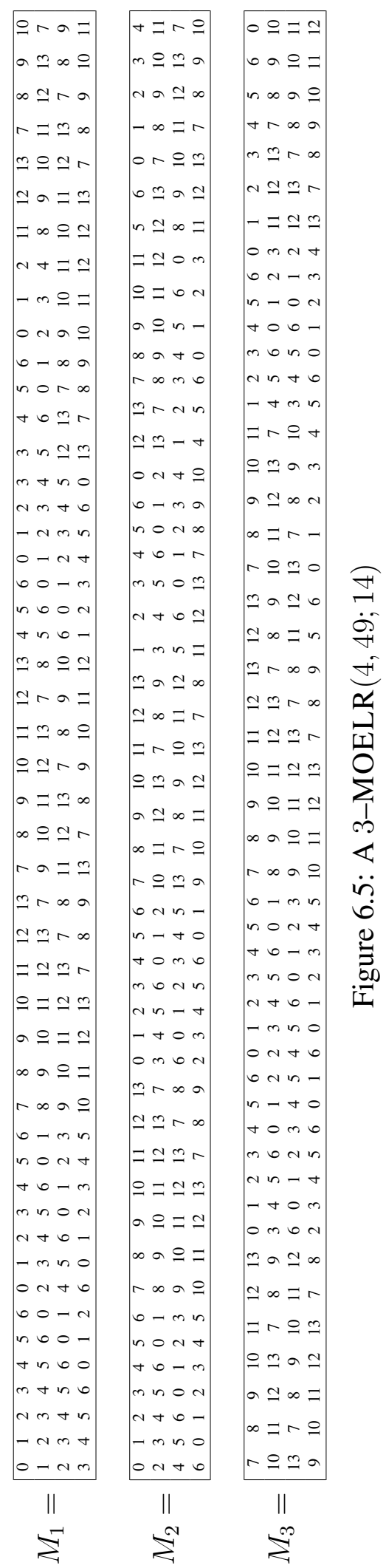




\subsection{Computer Construction of MOELR}

The construction of MOELRs given in Examples 6.5 and 6.6 was accomplished by a program coded in $\mathrm{C}++$. The following is the description of the different parts of the programs created.

Note that the programs were designed only to find $3-\operatorname{MOELR}(a, b ; n)$. The program was separated into 2 stages: a first stage program that generated the triples used in the 3 corresponding matrices and a second stage program to place the triples in matrices, check which are unique, and record unique up to isomorphism 3-MOELR in a list.

The second program used recursion to exhaustively generate all possible combinations of entries in the matrix. Once a single solution was found, it was printed and placed in a set that could be called later to check whether any new solution was isomorphic to a previous solution. To check for isomorphisms, the new solution and all old solutions were permuted in a way that set the first column and first row in lexicographical order. Once in lexicographical order, the two solutions were compared to see if a one to one mapping exists. Note that only a single set of triples from the first program was used to generate all the solutions. That means, even if the program found all possible solutions for this triple set, there are more triple sets that could be used.

These programs were used to find a $3-\operatorname{MOELR}(3,12 ; 6)$ and a $3-\operatorname{MOELR}(4,9 ; 6)$.

\subsection{Supporting Lemmas}

Notice that if we take the transpose of each rectangle in a $k$-MOELR $(a, b ; n)$, then the equitability and orthogonality properties still hold. Thus we have the following result.

Lemma 6.9. If there exists a $k-\operatorname{MOELR}(a, b ; n)$, then there exists a $k-\operatorname{MOELR}(b, a ; n)$.

The next result is very similar to the result for MOLS that says that if there exists $k-\operatorname{MOLS}(n)$, then $k \leq n-1$ (see [19]).

Lemma 6.10. If there exists a $k-\operatorname{MOELR}(a, b ; n)$, then $k \leq n-1$.

Proof: Without loss, we can assume that the first $n$ entries in the first row of each rectangle is $1,2, \cdots, n$ (i.e. $L_{j}(1, i)=i$ is the first row and $i^{t h}$ column in the $j^{\text {th }}$ rectangle for $i=1,2, \cdots, n$ and $j=1,2, \cdots, k)$. Then $L_{j}(2,1) \neq 1$ for all $j=1,2, \cdots, k$, because 1 has already appeared in the first column of every rectangle. Let $L_{1}(2,1)=x_{1}$. Then $L_{j}(2,1) \neq x_{1}$ for all $j=2,3, \cdots, k$ because the ordered pair $\left(x_{1}, x_{1}\right)$ has already appeared for each pair of rectangles in row 1 (i.e. $L_{j}(1, i)=L_{h}(1, i)=i$ for all $j, h \in\{1,2, \cdots, k\}$ and $i=1,2, \cdots, n)$. Furthermore, $L_{j}(2,1) \neq L_{h}(2,1)$ for all $j, h \in\{2,3, \cdots, k\}$ for the same reason. Thus we have that each entry $L_{j}(2,1)$ is unique and also $L_{j}(2,1) \neq 1$ for all $j=1,2, \cdots, k$. Because there are only $n-1$ ways to fill this entry in each rectangle, it follows that we can have at most $n-1$ rectangles. Thus $k \leq n-1$.

Lemma 6.11. If there exists $k-\operatorname{MOLS}(n)$ and $a \mid n$, then there exists a $k-\operatorname{MOELR}(a, b ; n)$. 
Proof: Suppose $a \leq b$ and $a \mid n$. Write $n=a x$ so $a b=n^{2}=a^{2} x^{2}$; and therefore, $b=a x^{2}=x n$. Let $A_{1}, A_{2}, \cdots, A_{k}$ be $k-\operatorname{MOLS}(n)$. We form the $j^{\text {th }}$ rectangle $M_{j}$ as follows.

$$
\begin{aligned}
& M_{j}(i, t n+l)=A_{j}(t a+i, l) \\
& \text { for } j=1,2, \cdots, k ; \quad i=1,2, \cdots, a ; \quad t=0,1, \cdots, x-1 ; \quad l=1,2, \cdots, n
\end{aligned}
$$

This rectangle has $a$ rows and $b=x n$ columns. Every symbol appears in each row $x$ times and each column 0 or 1 times. Furthermore, every ordered pair occurs exactly once. Therefore, the $M_{j}$ for $j=1,2, \cdots, k$ form a $k-\operatorname{MOELR}(a, b ; n)$.

Example 6.12. A 2-MOELR $(6,24 ; 12)$ using Lemma 6.11

We will construct a $2-\operatorname{MOELR}(6,24 ; 12)$ instead of the $5-\operatorname{MOELR}(6,24 ; 12)$ to make the example easier to understand. Following are the 2-MOLS(12).

$A_{1}=$\begin{tabular}{|l|}
$0123456789 a b$ \\
$123450789 a b 6$ \\
$23450189 a b 67$ \\
$3450129 a b 678$ \\
$450123 a b 6789$ \\
$501234 b 6789 a$ \\
\hline $6789 a b 012345$ \\
$789 a b 6123450$ \\
$89 a b 67234501$ \\
$9 a b 678345012$ \\
$a b 6789450123$ \\
$b 6789 a 501234$
\end{tabular} \mid $A_{2}=$\begin{tabular}{l}
$03619 b 28547 a$ \\
$1472 a 639058 b$ \\
$2583 b 74 a 1096$ \\
$3094685 b 21 a 7$ \\
$41 a 5790632 b 8$ \\
$52 b 08 a 174369$ \\
\hline $69073582 b a 14$ \\
$7 a 1840936 b 25$ \\
$8 b 2951 a 47630$ \\
$963 a 02 b 58741$ \\
$a 74 b 13609852$ \\
$b 8562471 a 903$ \\
\hline
\end{tabular}

By performing Lemma 6.11 we adjoin the last six rows to the right of the first six rows to get a total of 6 rows and 24 columns. In Figure 6.6, we have 2-MOELR(6, 24; 12$)$.

$M_{1}=$\begin{tabular}{|l|l|}
\hline $0123456789 a b$ & $6789 a b 012345$ \\
$123450789 a b 6$ & $789 a b 6123450$ \\
$23450189 a b 67$ & $89 a b 67234501$ \\
$3450129 a b 678$ & $9 a b 678345012$ \\
$450123 a b 6789$ & $a b 6789450123$ \\
$501234 b 6789 a$ & $b 6789 a 501234$
\end{tabular}$\quad M_{2}=$\begin{tabular}{|l|l|}
$03619 b 28547 a$ & $69073582 b a 14$ \\
$1472 a 639058 b$ & $7 a 1840936 b 25$ \\
$2583 b 74 a 1096$ & $8 b 2951 a 47630$ \\
$3094685 b 21 a 7$ & $963 a 02 b 58741$ \\
$41 a 5790632 b 8$ & $a 74 b 13609852$ \\
$52 b 08 a 174369$ & $b 8562471 a 903$ \\
\hline
\end{tabular}

Figure 6.6: A 2-MOELR $(6,24 ; 12)$

MacNeish [33] and Mann [34] showed that the maximum number of $\operatorname{MOLS}\left(p^{(i+j) / 2}\right)$ is $p^{(i+j) / 2}-1$. Therefore, by applying Lemma 6.11, we have the following result.

Corollary 6.13. There exists a complete set of mutually orthogonal equitable rectangles, or a $\left(p^{(i+j) / 2}-1\right)-\operatorname{MOELR}\left(p^{i}, p^{j} ; p^{i+j}\right)$ for all $i, j \geq 0$ and $p$ a prime. 
The next two results rely on the existence of orthogonal arrays. An orthogonal array, $\mathrm{OA}(k, n)$, is equivalent to $(k-2)-\operatorname{MOLS}(n)$ (see [19]).

Lemma 6.14. If there exists an $\mathrm{OA}\left(k, n_{1}\right)$ and there exists a set of $k-\operatorname{MOLS}\left(n_{2}\right)$, then there exists a $k$-MOELR $\left(n_{2}, n_{1}^{2} n_{2} ; n_{1} n_{2}\right)$.

Proof: Let $M_{i, j}$ represent the $i^{\text {th }}$ square of the set of $k$-MOLS $\left(n_{2}\right)$ on the $j^{\text {th }}$ set of $n_{2}$ symbols, where $j=1,2, \cdots, n_{1}$ and $i=1,2, \ldots, k$. Form the array $X$ as follows. On each entry of the $\mathrm{OA}\left(k, n_{1}\right), O_{i, s}$, replace $O_{i, s}$ with $M_{i, O_{i, s}}$ for $i=1,2, \ldots, k$ and $s=$ $1,2, \cdots, n_{1}^{2}$. Now each row of $X$ corresponds to an $n_{2} \times n_{1}^{2} n_{2}$ rectangle on $n_{1} n_{2}$ symbols.

For any two rows of $X, i_{1}, i_{2}=1, \ldots, k$, we have every ordered pair $\left(M_{i_{1}, j_{1}} M_{i_{2}, j_{2}}\right)$ for $j_{1}, j_{2} \in\left\{1,2, \ldots, n_{1}\right\}$ exactly once because $X$ is an $\mathrm{OA}\left(k, n_{1}\right)$. Furthermore, because $M_{i_{1}, j_{1}}$ and $M_{i_{2}, j_{2}}$ are orthogonal, we see every ordered pair among their $n_{2}$ symbols exactly once. Therefore, we see every ordered pair on $n_{1} n_{2}$ symbols exactly once among the two rows of $X$. Consider some row $i$ of $X$. This row corresponds to one of the $n_{2} \times n_{1}^{2} n_{2}$ rectangles. Each $M_{i, j}$ has $n_{2}$ rows and it was part of the set of $k$-MOLS $\left(n_{2}\right)$, so each symbol occurs exactly once in each column. Furthermore, each $M_{i, j}$ is repeated $n_{1}$ times for $j=1,2, \ldots, n_{1}$. Therefore, each symbol occurs exactly $n_{1}$ times in each row. Thus, the $k$ rows of $X$ form a $k-\operatorname{MOELR}\left(n_{2}, n_{1}^{2} n_{2} ; n_{1} n_{2}\right)$.

Example 6.15. A 3-MOELR $(5,20 ; 10)$ using Lemma 6.14

Because there exists 2-MOLS $(10)$, we could use Theorem 6.11 to obtain a $2-\operatorname{MOELR}(5,20 ; 10)$. However, there exists an $\mathrm{OA}(3,2)$ and there exists $3-\operatorname{MOLS}(5)$. Therefore, we can apply Theorem 6.14 to obtain a $3-\operatorname{MOELR}(5,20 ; 10)$. We construct the $3-\operatorname{MOELR}(5,20 ; 10)$ as follows. Suppose that the 3 squares of the 3-MOLS $(5)$ are labeled $M_{1,1}, M_{2,1}, M_{3,1}$ and are defined on symbol set $S_{1}=\{1,2,3,4,5\}$. Consider another copy of this set of MOLS on the symbol set $S_{2}=\left\{1^{\prime}, 2^{\prime}, 3^{\prime}, 4^{\prime}, 5^{\prime}\right\}$. So we have the copies $M_{1,2}, M_{2,2}, M_{3,2}$.

Let $O$ be the given $\mathrm{OA}(3,2)$.

$$
O=\mathrm{OA}(3,2)=\left[\begin{array}{llll}
1 & 1 & 2 & 2 \\
1 & 2 & 1 & 2 \\
1 & 2 & 2 & 1
\end{array}\right]
$$

Following the proof of Theorem 6.14, we replace each entry of $O_{i, s}$ with $M_{i, O_{i, s}}$ for $i=$ $1,2,3$ and $s=1,2,3,4$. So,

$$
O=\mathrm{OA}(3,2)=\left[\begin{array}{llll}
1 & 1 & 2 & 2 \\
1 & 2 & 1 & 2 \\
1 & 2 & 2 & 1
\end{array}\right] \Rightarrow X=\left[\begin{array}{llll}
M_{1,1} & M_{1,1} & M_{1,2} & M_{1,2} \\
M_{2,1} & M_{2,2} & M_{2,1} & M_{2,2} \\
M_{3,1} & M_{3,2} & M_{3,2} & M_{3,1}
\end{array}\right]
$$

Lemma 6.16. Suppose there exists a set of $3-\operatorname{MOLS}(s)$. Write $s=a y+r$ for some $y \in \mathbb{Z}$ and $0 \leq r<a$. Then if $n=2 s$ and $r=\frac{a}{4}$ or $r=\frac{3 a}{4}$, then there exists $a 3-\operatorname{MOELR}(a, b ; n)$ for all $a<s$ and $s \mid b$. 
Proof: We have that $a b=n^{2}=4 s^{2}$. Therefore $b=\frac{4 s^{2}}{a}=\frac{4 s \cdot s}{a}$. Because $s \mid b$, we have $\frac{b}{s}=\frac{4 s}{a} \in \mathbb{Z}$. Now, because $\frac{4 s}{a} \in \mathbb{Z}$, it follows that

$$
\begin{aligned}
\frac{4(a y+r)}{a} \in \mathbb{Z} & \Rightarrow \frac{4 a y}{a}+\frac{4 r}{a} \in \mathbb{Z} \\
& \Rightarrow \frac{4 r}{a} \in \mathbb{Z} \\
& \Rightarrow r \cdot \frac{4}{a}=\frac{r}{a / 4}
\end{aligned}
$$

Because $4 \mid a$, we have that $\frac{a}{4} \mid r$. In our case, $r=\frac{a}{4}$ or $r=\frac{3 a}{4}$.

Given a set of 3-MOLS $(s)$, we do the following. Let $S_{1}$ and $S_{2}$ be a partition of a set of $n$ symbols, where $\left|S_{j}\right|=s$ for $j=1,2$. Define $M_{i, j}$ to be the $i^{t h}$ Latin square of the set of $3-\operatorname{MOLS}(s)$ on symbol set $S_{j}$. Let $\mathcal{R}_{1}, \mathcal{R}_{2}, \mathcal{R}_{3}$ be a $3-\operatorname{MOELR}(s, 4 s ; 2 s)$ constructed by applying Lemma 6.14 with $k=3, n_{1}=2$, and $n_{2}=s$.

Let $X=\left[\begin{array}{lll}\mathcal{R}_{1} & \mathcal{R}_{2} & \mathcal{R}_{3}\end{array}\right]^{T}$ be an array where the rows form this $3-\operatorname{MOELR}(s, 4 s ; 2 s)$. Thus

$$
X=\begin{array}{|l|}
\hline \mathcal{R}_{1} \\
\hline \mathcal{R}_{2} \\
\hline \mathcal{R}_{3} \\
\hline M_{1,1}
\end{array}=\begin{array}{|l|l|l|l|}
\hline M_{1,1} & M_{1,2} & M_{1,2} \\
\hline M_{2,1} & M_{2,2} & M_{2,1} & M_{2,2} \\
\hline M_{3,1} & M_{3,2} & M_{3,2} & M_{3,1} \\
\hline
\end{array}
$$

Consider row $\mathcal{R}_{i}$ of $X$. For $t=1,2, \ldots, s$, let $r_{t, j}$ denote the $t^{t h}$ row of $M_{i, j}$. For example, we write $\mathcal{R}_{1}$ as in Figure 6.7

We have that $\mathcal{R}_{i}$ is an $s \times 4$ array where each column represents a $\operatorname{LS}(s)$. Because $s=a y+r$, it follows that we can rearrange each column of $\mathcal{R}_{i}$ into a new structure which is an $a \times y$ array followed by an $r \times 1$ array.

Let $\mathcal{R}_{i}^{\prime}$ be this arrangement of columns of $\mathcal{R}_{i}$. We give $\mathcal{R}_{1}^{\prime}$ in Figure 6.8 .

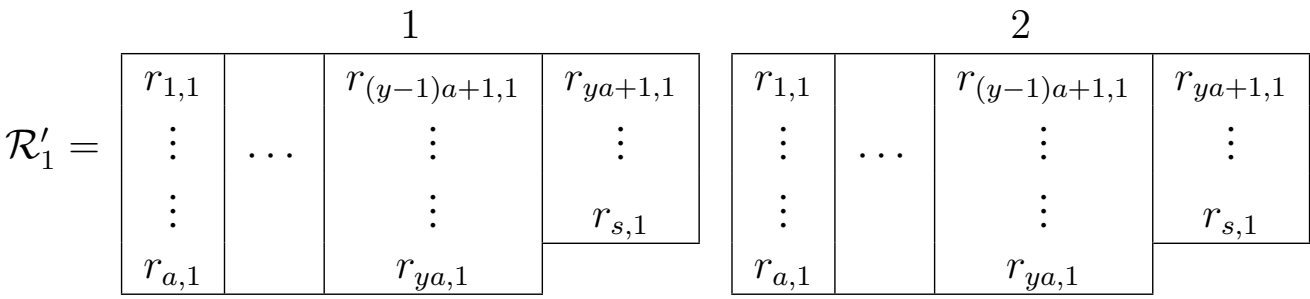

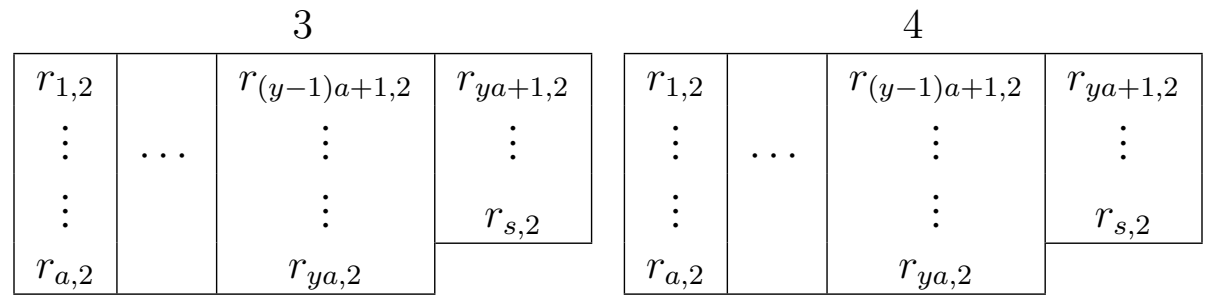

Figure 6.8: $\mathcal{R}_{1}$ of matrix $X$. 


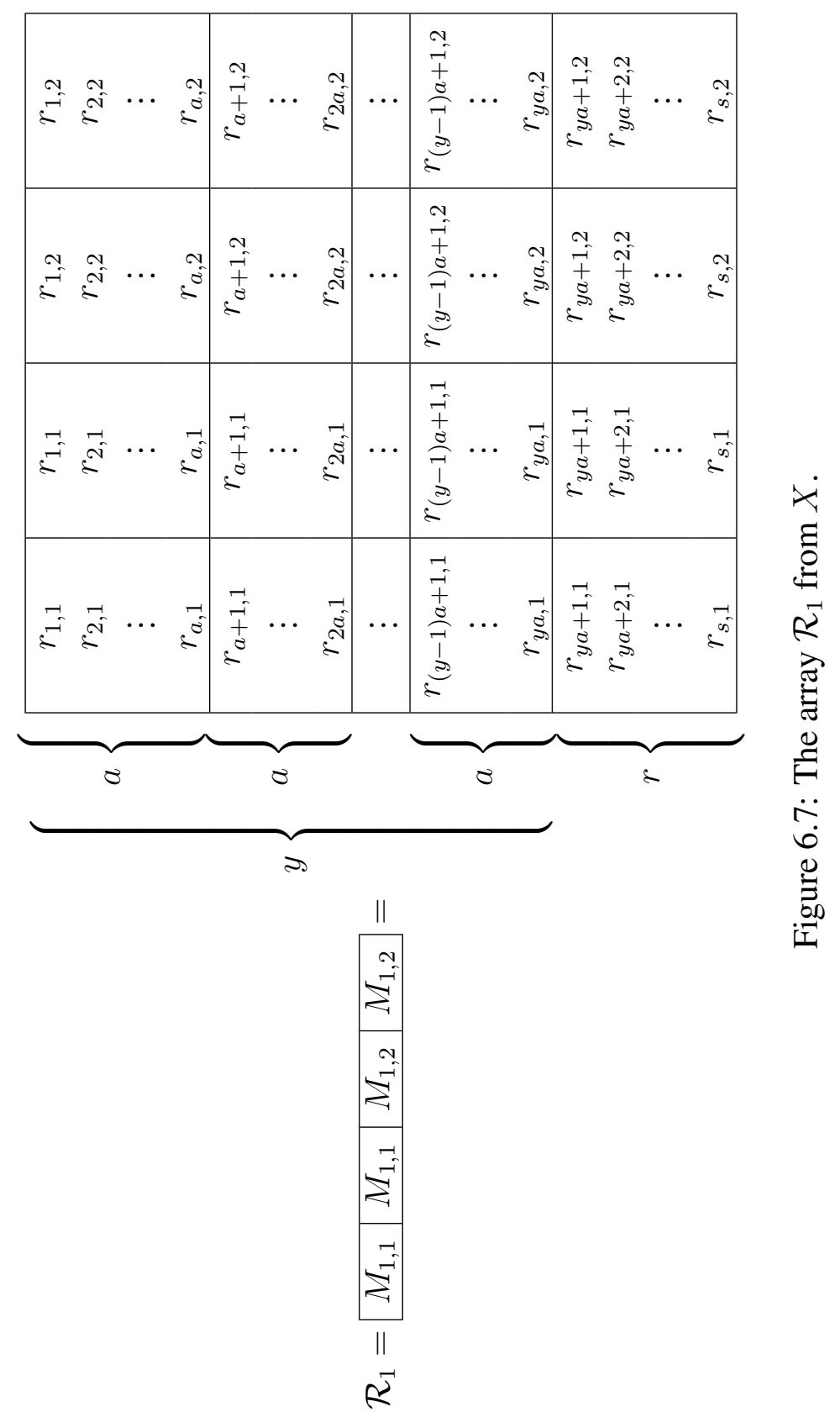


Let $\mathcal{R}_{i, j}^{\prime}$ be the $a \times y$ array from column $j$ of $\mathcal{R}_{i}$. Let $z_{i, j}$ be the $r \times 1$ array from column $j$ of $\mathcal{R}_{i}$.

Let $v$ be the $4 s$-tuple obtained by taking each entry from $\mathcal{R}_{i}^{\prime}$ going down the columns. Thus, from $\mathcal{R}_{i}^{\prime}$ we get

$$
v=\left(r_{1,1}, r_{2,1}, \ldots, r_{s, 1}, r_{1,1}, \ldots, r_{s, 1}, r_{1,2}, \ldots, r_{s, 2}, r_{1,2}, \ldots, r_{s, 2}\right) .
$$

Now write $v$ as $v=\left(v_{0}, v_{1}, \ldots, v_{\frac{4 s}{a}-1}\right)$ where each $v_{i}$ has $a$ entries.

Let $\mathcal{R}_{i}^{\prime \prime}=\left(v_{0}^{T}, v_{1}^{T}, \ldots, v_{\frac{4 s}{a}-1}^{T}\right)$. We now show that $\mathcal{R}_{1}^{\prime \prime}, \mathcal{R}_{2}^{\prime \prime}, \mathcal{R}_{3}^{\prime \prime}$ form a $3-\operatorname{MOELR}(a, b ; n)$. First note that $\mathcal{R}_{i}^{\prime \prime}$ is an $a \times \frac{4 s}{a}$ array. However, each entry represents a row of an $\operatorname{LS}(s)$. Thus, $\mathcal{R}_{i}^{\prime \prime}$ contains $\left(\frac{4 s}{a}\right) s=\frac{4 s^{2}}{a}=b$ columns. Because we apply the same structuring to each $\mathcal{R}_{i}$, it follows that orthogonality holds.

Case 1: $r=\frac{a}{4}$

We have that $\mathcal{R}_{i}^{\prime \prime}$ has the following form.

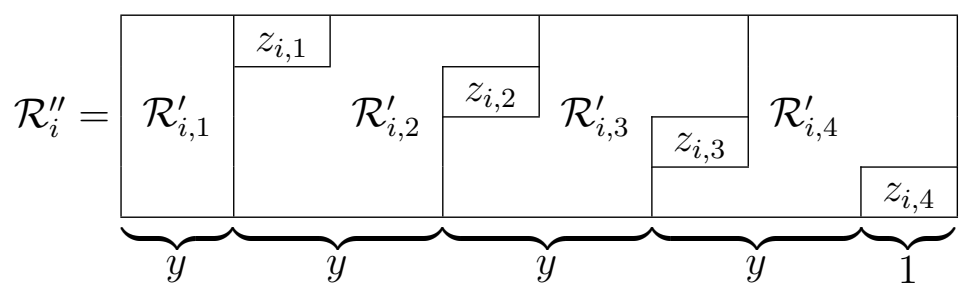

Thus the number of columns in $\mathcal{R}_{i}^{\prime \prime}$ is

$$
s(4 y+1)=s\left(4\left(\frac{s-r}{a}\right)+1\right)=s\left(\frac{4 s}{a}-\frac{4 r}{a}+1\right)=\frac{4 s^{2}}{a}=b
$$

It is easy to see that equitability holds in each column because each $z_{i, j}$ lies in a different column. Because $a<s$, it follows that each symbol appears exactly 0 or 1 time in each column.

Consider any row of $\mathcal{R}_{i}^{\prime \prime}$. In this row, each symbol appears $2 y+1$ or $2 y$ times. We have:

$$
2 y=2\left(\frac{s-r}{a}\right)=\frac{2 s}{a}-\frac{2 r}{a}=\frac{2 s}{a}-\frac{2}{a} \cdot \frac{a}{4}=\frac{2 s}{a}-\frac{1}{2}=\left\lfloor\frac{2 s}{a}\right\rfloor=\left\lfloor\frac{b}{n}\right\rfloor
$$

and

$$
2 y+1=2\left(\frac{s-r}{a}\right)+1=\frac{2 s}{a}-\frac{2 r}{a}+1=\frac{2 s}{a}-\frac{1}{2}+1=\frac{2 s}{a}+\frac{1}{2}=\left\lceil\frac{2 s}{a}\right\rceil=\left\lceil\frac{b}{n}\right\rceil,
$$

thus equitability holds.

Case 2: $\quad r=\frac{3 a}{4}$

In this case, $\mathcal{R}_{i}^{\prime \prime}$ has the following form. 


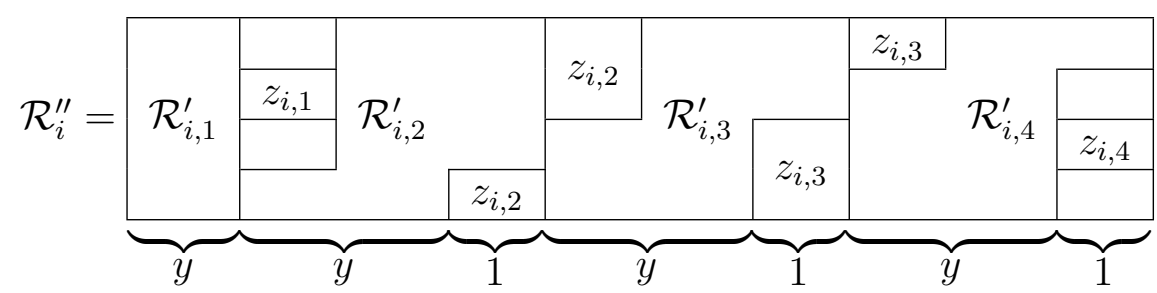

Thus the number of columns in $\mathcal{R}_{i}^{\prime \prime}$ is

$$
s(4 y+3)=s\left(4\left(\frac{s-r}{a}\right)+3\right)=s\left(\frac{4 s}{a}-\frac{4 r}{a}+3\right)=\frac{4 s^{2}}{a}=b .
$$

Again, it is easy to see that equitability holds in each column. As in Case 1, each symbol appears $2 y$ or $2 y+1$ times in each row.

Lemma 6.17. Suppose there exists a set of $3-\operatorname{MOLS}(s)$. Write $s=$ ay $+r$ for some $y \in \mathbb{Z}$ and $0 \leq r<a$. Then if $n=3 s$ and $r=\frac{a}{9}$ or $r=\frac{8 a}{9}$, then there exists a $3-\operatorname{MOELR}(a, b ; n)$ for all $a<s$ and $s \mid b$.

Proof: We have that $a b=n^{2}=9 s^{2}$. Therefore $b=\frac{9 s^{2}}{a}=\frac{9 s \cdot s}{a}$ Because $s \mid b$, we have $\frac{b}{s}=\frac{9 s}{a} \in \mathbb{Z}$. Now, because $\frac{9 s}{a} \in \mathbb{Z}$, it follows that

$$
\begin{aligned}
\frac{9(a y+r)}{a} \in \mathbb{Z} & \Rightarrow \frac{9 a y}{a}+\frac{9 r}{a} \in \mathbb{Z} \\
& \Rightarrow \frac{9 r}{a} \in \mathbb{Z} \\
& \Rightarrow r \cdot \frac{9}{a}=\frac{r}{a / 9}
\end{aligned}
$$

Because $9 \mid a$, we have that $\frac{a}{9} \mid r$. In our case, $r=\frac{a}{9}$ or $r=\frac{8 a}{9}$. Given a set of 3-MOLS $(s)$, we do the following. Let $S_{1}, S_{2}$ and $S_{3}$ be a partition of $n$ symbols, where $\left|S_{i}\right|=s$ for $i=1,2,3$.

Define $M_{i, j}$ to be the $i^{\text {th }}$ Latin square of the set of 3-MOLS $(s)$ on the symbol set $S_{j}$. Let $\mathcal{R}_{1}, \mathcal{R}_{2}, \mathcal{R}_{3}$ be a $3-\operatorname{MOELR}(s, 9 s ; 3 s)$ constructed by applying Lemma 6.14 with $k=3, n_{1}=3$, and $n_{2}=s$.

Let $X=\left[\begin{array}{lll}\mathcal{R}_{1} & \mathcal{R}_{2} & \mathcal{R}_{3}\end{array}\right]^{T}$ be an array where the rows form a $3-\operatorname{MOELR}(s, 9 s ; 3 s)$. Thus

$$
X=\begin{array}{|l|l|l|l|l|l|l|l|l|l|}
\hline \mathcal{R}_{1} \\
\hline \mathcal{R}_{2} \\
\hline \mathcal{R}_{3} \\
\hline M_{1,1} & M_{1,1} & M_{1,1} & M_{1,2} & M_{1,2} & M_{1,2} & M_{1,3} & M_{1,3} & M_{1,3} \\
\hline M_{2,1} & M_{2,2} & M_{2,3} & M_{2,1} & M_{2,2} & M_{2,3} & M_{2,1} & M_{2,2} & M_{2,3} \\
\hline M_{3,1} & M_{3,2} & M_{3,3} & M_{3,2} & M_{3,3} & M_{3,1} & M_{3,3} & M_{3,1} & M_{3,2} \\
\hline
\end{array}
$$

Consider row $\mathcal{R}_{i}$ of $X$. For $t=1,2, \ldots, s$, let $r_{t, j}$ denote the $t^{t h}$ row of $M_{i, j}$.

For example, we write $\mathcal{R}_{1}$ as in Figure 6.9.

We have that $\mathcal{R}_{i}$ is an $s \times 9$ array where each column represents an $\operatorname{LS}(s)$. Because $s=a y+r$, it follows that we can rearrange each column of $\mathcal{R}_{i}$ into a new structure which 


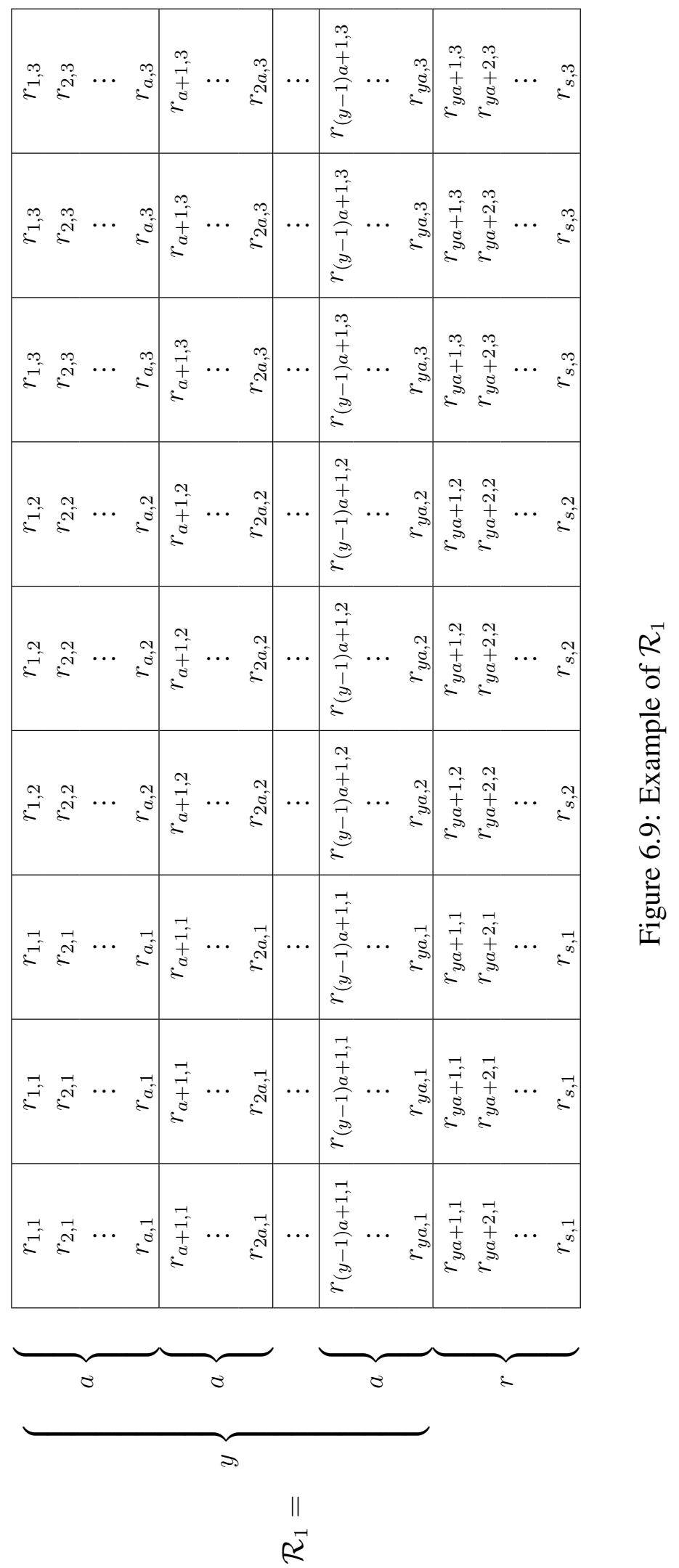


is an $a \times y$ array followed by an $r \times 1$ array. Let $\mathcal{R}_{i}^{\prime}$ be this arrangement of columns of $\mathcal{R}_{i}$. We give $\mathcal{R}_{1}^{\prime}$ in Figure 6.10
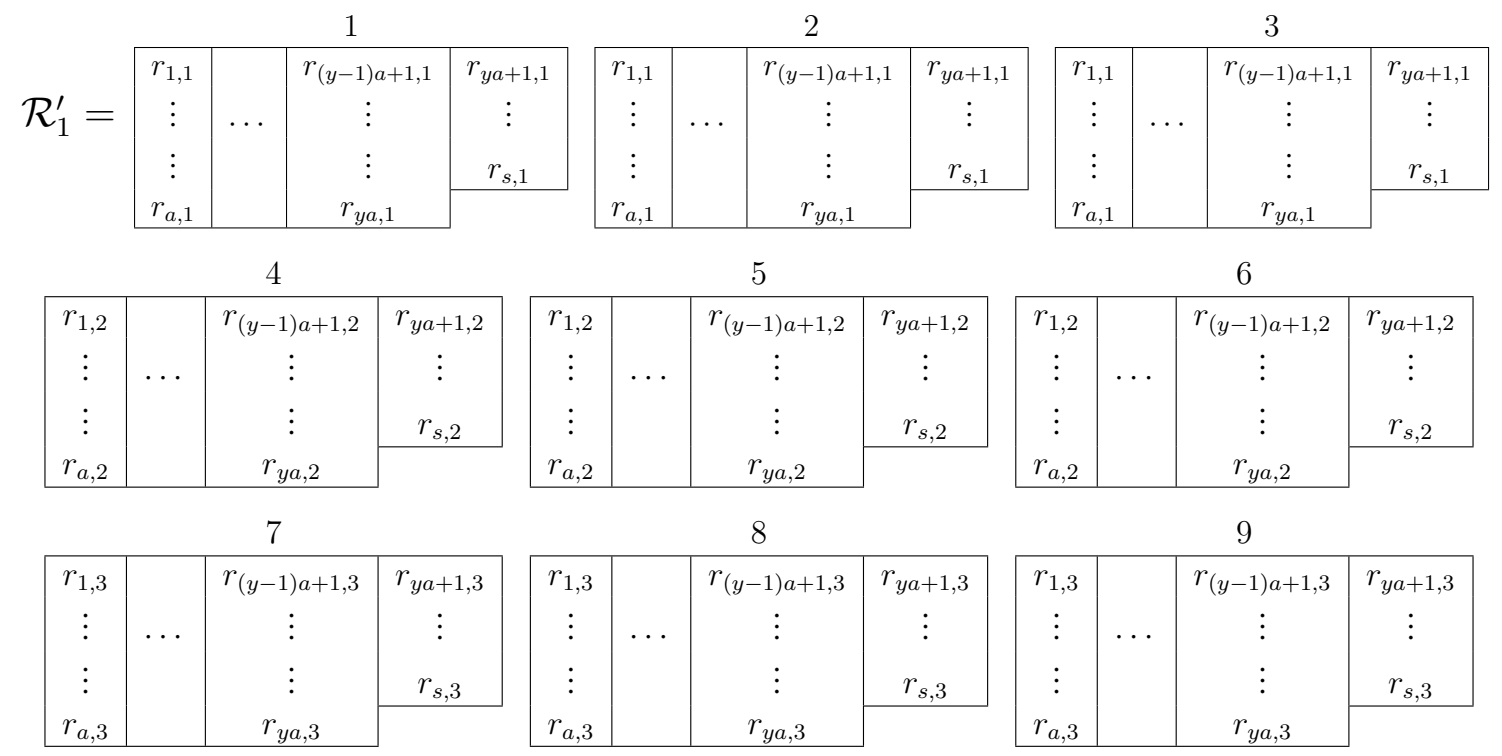

Figure 6.10: $\mathcal{R}_{1}$ of matrix $X$.

Let $\mathcal{R}_{i, j}^{\prime}$ be the $a \times y$ array from column $j$ of $\mathcal{R}_{i}$. Let $z_{i, j}$ be the $r \times 1$ array from column $j$ of $\mathcal{R}_{i}$. Let $v$ be the $9 s$-tuple obtained by taking each entry from $\mathcal{R}_{i}^{\prime}$ going down the columns. Thus, from $\mathcal{R}_{1}^{\prime}$ we get

$$
v=\left(r_{1,1}, r_{2,1} \ldots, r_{s, 1}, \ldots, r_{1,3}, \ldots, r_{s, 3}\right)
$$

Now write $v$ as $v=\left(v_{0}, v_{1}, \ldots, v_{\frac{9 s}{a}-1}\right)$ where each $v_{i}$ has $a$ entries.

Let $\mathcal{R}_{i}^{\prime \prime}=\left(v_{0}^{T}, \ldots, v_{\frac{9 s}{a}-1}^{T}\right)$. Now we show that $\mathcal{R}_{1}^{\prime \prime}, \mathcal{R}_{2}^{\prime \prime}, \mathcal{R}_{3}^{\prime \prime}$ form a $3-\operatorname{MOELR}(a, b ; n)$. First note that $\mathcal{R}_{1}^{\prime \prime}$ is an $a \times \frac{9 s}{a}$ array. However, each entry represents a row of an $\operatorname{LS}(s)$. Thus, $\mathcal{R}_{i}^{\prime \prime}$ contains $\left(\frac{9 s}{a}\right) s=\frac{9 s^{2}}{a}=b$ columns. Because we apply the same structuring to each $\mathcal{R}_{i}$, it follows that orthogonality holds.

Case 1: $\quad r=\frac{a}{9}$

We have that $\mathcal{R}_{i}^{\prime \prime}$ has the form in Figure 6.11. Thus the number of columns in $\mathcal{R}_{i}^{\prime \prime}$ is

$$
s(9 y+1)=s\left(9\left(\frac{s-r}{a}\right)+1\right)=s\left(\frac{9 s}{a}-\frac{9 r}{a}+1\right)=\frac{9 s^{2}}{a}=b .
$$

It is easy to see that equitability holds in each column because each $z_{i, j}$ lies in a different column. Because $a<s$, it follows that each symbol appears exactly 0 or 1 time in each 


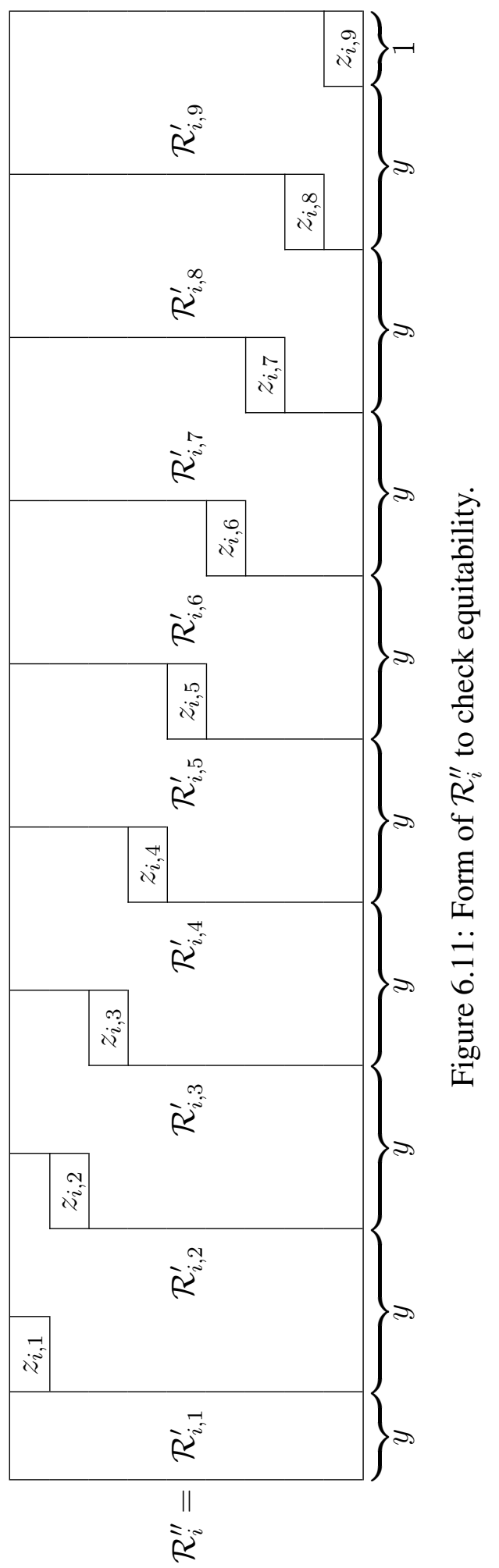


column. Consider any row of $\mathcal{R}_{i}^{\prime \prime}$. In this row, each symbol appears $3 y+1$ or $3 y$ times. We have:

$$
\begin{aligned}
3 y & =3\left(\frac{s-r}{a}\right) \\
& =\frac{3 s}{a}-\frac{3 r}{a} \\
& =\frac{3 s}{a}-\frac{3}{a} \cdot \frac{a}{9} \\
& =\frac{3 s}{a}-\frac{1}{9} \\
& =\left\lfloor\frac{3 s}{a}\right\rfloor=\left\lfloor\frac{b}{n}\right\rfloor
\end{aligned}
$$

and

$$
\begin{aligned}
3 y+1 & =3\left(\frac{s-r}{a}\right)+1 \\
& =\frac{3 s}{a}-\frac{3 r}{a}+1 \\
& =\frac{3 s}{a}-\frac{1}{9}+1 \\
& =\frac{3 s}{a}+\frac{8}{9} \\
& =\left\lceil\frac{3 s}{a}\right\rceil=\left\lceil\frac{b}{n}\right\rceil,
\end{aligned}
$$

thus equitability holds.

Case 2: $\quad r=\frac{8 a}{9}$

In this case, $\mathcal{R}_{i}^{\prime \prime}$ has the form in Figure 6.12. This is because the number of columns in $\mathcal{R}_{i}^{\prime \prime}$ is

$$
s(9 y+8)=s\left(9\left(\frac{s-r}{a}\right)+8\right)=s\left(\frac{9 s}{a}-\frac{9 r}{a}+8\right)=\frac{9 s^{2}}{a}=b .
$$

Again, it is easy to see that equitability holds in each column. As in Case 1, each symbol appears $3 y$ or $3 y+1$ times in each row.

Lemma 6.18. Suppose there exists a set of $3-\operatorname{MOLS}(s)$. Write $s=a y+r$ for some $y \in \mathbb{Z}$ and $0 \leq r<a$. Then if $n=6 s$ and $r=\frac{a}{36}$ or $r=\frac{35 a}{36}$, then there exists $a$ $3-\operatorname{MOELR}(a, b ; n)$ for all $a<s$ and $s \mid b$. 


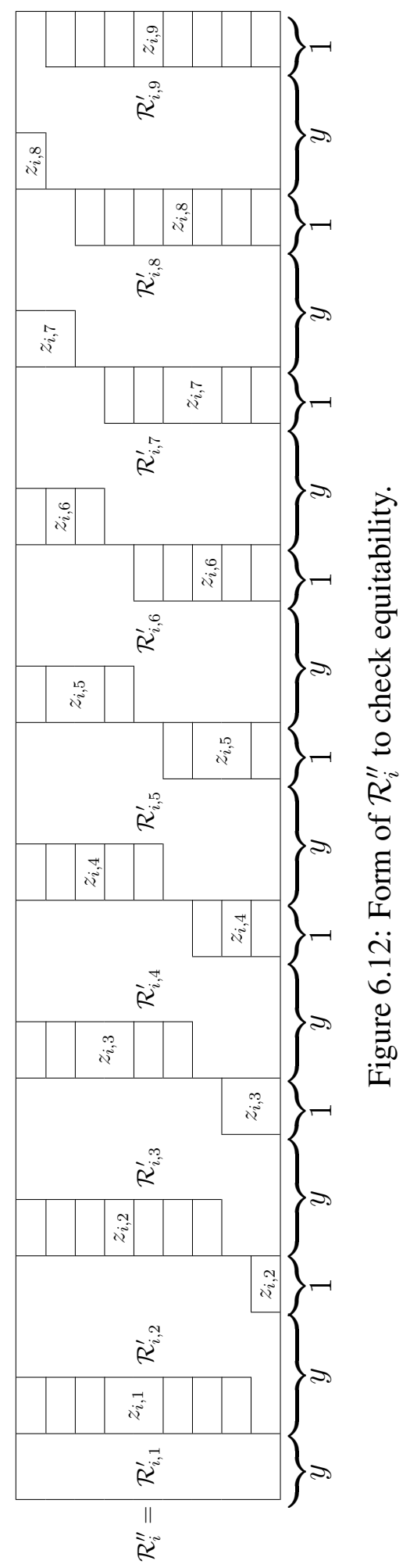


Proof: We have that $a b=n^{2}=36 s^{2}$. Therefore $b=\frac{36 s^{2}}{a}=\frac{36 s \cdot s}{a}$ Because $s \mid b$, we have $\frac{b}{s}=\frac{36 s}{a} \in \mathbb{Z}$. Now, because $\frac{36 s}{a} \in \mathbb{Z}$, it follows that

$$
\begin{aligned}
\frac{36(a y+r)}{a} \in \mathbb{Z} & \Rightarrow \frac{36 a y}{a}+\frac{36 r}{a} \in \mathbb{Z} \\
& \Rightarrow \frac{36 r}{a} \in \mathbb{Z} \\
& \Rightarrow r \cdot \frac{36}{a}=\frac{r}{a / 36}
\end{aligned}
$$

Because $36 \mid a$, we have that $\frac{a}{36} \mid r$. In our case, $r=\frac{a}{36}$ or $r=\frac{35 a}{36}$. Given a set of 3-MOLS $(s)$, we do the following. Let $S_{1}, S_{2}$ and $S_{3}$ be a partition of $n$ symbols, where $\left|S_{j}\right|=s$ for $j=1,2,3$.

Define $M_{i, j}$ to be the $i^{\text {th }}$ Latin square of the set of 3-MOLS $(s)$ on the symbol set $S_{j}$. Let $\mathcal{R}_{1}, \mathcal{R}_{2}, \mathcal{R}_{3}$ be a $3-\operatorname{MOELR}(s, 36 s ; 6 s)$ constructed by applying Lemma 6.14 with $k=3, n_{1}=6$, and $n_{2}=s$.

Let $X=\left[\begin{array}{lll}\mathcal{R}_{1} & \mathcal{R}_{2} & \mathcal{R}_{3}\end{array}\right]^{T}$ be an array where the rows form a $3-\operatorname{MOELR}(s, 36 s ; 6 s)$. Thus

$X=$\begin{tabular}{|l|l|l|l|l|l|l|l|l|l|l|}
\hline $\mathcal{R}_{1}$ \\
\hline $\mathcal{R}_{2}$ \\
\hline $\mathcal{R}_{3}$ \\
\hline$M_{1,1}$ & $M_{1,1}$ & $M_{1,1}$ & $\cdots$ & $M_{1,6}$ & $M_{1,6}$ & $M_{1,6}$ & $M_{1,6}$ & $M_{1,6}$ & $M_{1,6}$ \\
\hline$M_{2,1}$ & $M_{2,2}$ & $M_{2,3}$ & $\cdots$ & $M_{2,1}$ & $M_{2,2}$ & $M_{2,3}$ & $M_{2,4}$ & $M_{2,5}$ & $M_{2,6}$ \\
\hline$M_{3,1}$ & $M_{3,2}$ & $M_{3,3}$ & $\cdots$ & $M_{3,2}$ & $M_{3,3}$ & $M_{3,4}$ & $M_{3,5}$ & $M_{3,6}$ & $M_{3,1}$ \\
\hline
\end{tabular}

Consider row $\mathcal{R}_{i}$ of $X$. For $t=1,2, \ldots, s$, let $r_{t, j}$ denote the $t^{\text {th }}$ row of $M_{i, j}$.

For example, we write $\mathcal{R}_{1}$ as in Figure6.13.

We have the $\mathcal{R}_{i}$ is an $s \times 4$ array where each column represents an $\operatorname{LS}(s)$. Because $s=a y+r$, it follows that we can rearrange each column of $\mathcal{R}_{i}$ into a new structure which is an $a \times y$ array followed by an $r \times 1$ array. Let $\mathcal{R}_{i}^{\prime}$ be this arrangement of columns of $\mathcal{R}_{i}$. We give $\mathcal{R}_{1}^{\prime}$ in Figure 6.14

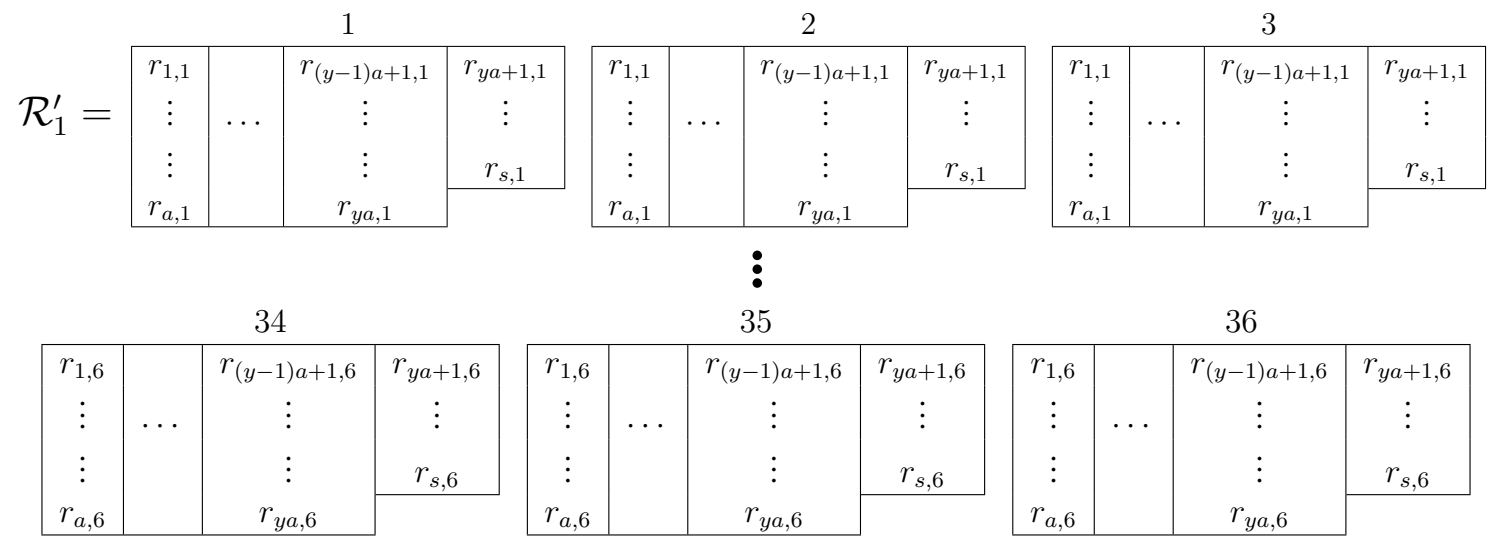

Figure 6.14: $\mathcal{R}_{1}$ of matrix $X$. 


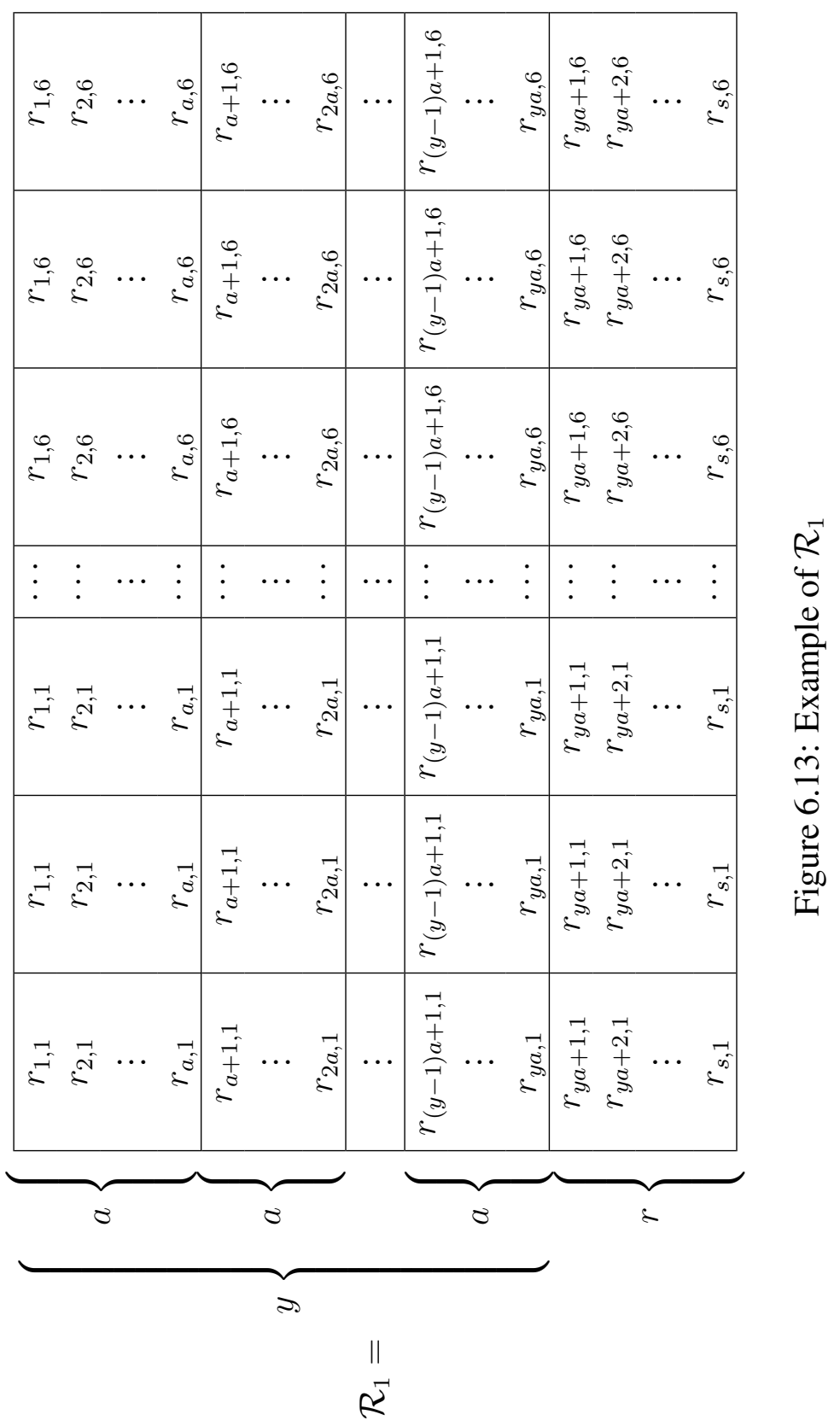


Let $\mathcal{R}_{i, j}^{\prime}$ be the $a \times y$ array from column $j$ of $\mathcal{R}_{i}$. Let $z_{i, j}$ be the $r \times 1$ array from column $j$ of $\mathcal{R}_{i}$. Let $v$ be the $9 s$-tuple obtained by taking each entry from $\mathcal{R}_{i}^{\prime}$ going down the columns. Thus, from $\mathcal{R}_{1}^{\prime}$ we get

$$
v=\left(r_{1,1}, r_{2,1} \ldots, r_{s, 1}, \ldots, r_{1,6}, \ldots, r_{s, 6}\right)
$$

Now write $v$ as $v=\left(v_{0}, v_{1}, \ldots, v_{\frac{36 s}{a}-1}\right)$ where each $v_{i}$ has $a$ entries.

Let $\mathcal{R}_{i}^{\prime \prime}=\left(v_{0}^{T}, v_{1}^{T}, \ldots, v_{\frac{36 s}{a}-1}^{T}\right)$. We now show that $\mathcal{R}_{1}^{\prime \prime}, \mathcal{R}_{2}^{\prime \prime}, \mathcal{R}_{3}^{\prime \prime}$ form a $3-\operatorname{MOELR}(a, b ; n)$. First note that $\mathcal{R}_{1}^{\prime \prime}$ is an $a \times \frac{36 s}{a}$ array. However, each entry represents a row of an $\operatorname{LS}(s)$. Thus, $\mathcal{R}_{i}^{\prime \prime}$ contains $\left(\frac{36 s}{a}\right) s=\frac{36 s^{2}}{a}=b$ columns. Because we apply the same structuring to each $\mathcal{R}_{i}$, it follows that orthogonality holds.

Case 1: $\quad r=\frac{a}{36}$

We have that $\mathcal{R}_{i}^{\prime \prime}$ has the form similar to that of Figure 6.11. Thus the number of columns in $\mathcal{R}_{i}^{\prime \prime}$ is

$$
s(36 y+1)=s\left(36\left(\frac{s-r}{a}\right)+1\right)=s\left(\frac{36 s}{a}-\frac{36 r}{a}+1\right)=\frac{36 s^{2}}{a}=b .
$$

It is easy to see that equitability holds in each column because each $z_{i, j}$ lies in a different column. Because $a<s$, it follows that each symbol appears exactly 0 or 1 time in each column. Consider any row of $\mathcal{R}_{i}^{\prime \prime}$. In this row, each symbol appears $6 y+1$ or $6 y$ times. We have:

$$
\begin{aligned}
6 y & =6\left(\frac{s-r}{a}\right) \\
& =\frac{6 s}{a}-\frac{6 r}{a} \\
& =\frac{6 s}{a}-\frac{6}{a} \cdot \frac{a}{36} \\
& =\frac{6 s}{a}-\frac{1}{36} \\
& =\left\lfloor\frac{6 s}{a}\right\rfloor=\left\lfloor\frac{b}{n}\right\rfloor
\end{aligned}
$$


and

$$
\begin{aligned}
6 y+1 & =6\left(\frac{s-r}{a}\right)+1 \\
& =\frac{6 s}{a}-\frac{6 r}{a}+1 \\
& =\frac{6 s}{a}-\frac{1}{36}+1 \\
& =\frac{6 s}{a}+\frac{35}{36} \\
& =\left\lceil\frac{6 s}{a}\right\rceil=\left\lceil\frac{b}{n}\right\rceil,
\end{aligned}
$$

thus equitability holds.

Case 2: $\quad r=\frac{35 a}{36}$

In this case, $\mathcal{R}_{i}^{\prime \prime}$ has a form similar to that of Figure 6.12 . Thus the number of columns in $\mathcal{R}_{i}^{\prime \prime}$ is

$$
s(36 y+35)=s\left(36\left(\frac{s-r}{a}\right)+35\right)=s\left(\frac{36 s}{a}-\frac{36 r}{a}+35\right)=\frac{36 s^{2}}{a}=b .
$$

Again, it is easy to see that equitability holds in each column. As in Case 1, each symbol appears $6 y$ or $6 y+1$ times in each row.

Example 6.19. 2-MOELR $(4,81 ; 18)$ using Lemma 6.16

Let $A=\left\{a_{0}, \ldots, a_{8}\right\}$ and $B=\left\{b_{0}, \ldots, b_{8}\right\}$ where $A$ and $B$ are two unique symbol sets. We also will only use 2-MOLS $(9)$ to demonstrate the idea. Let $r_{i, j}$ be the $i^{\text {th }}$ row of the $9 \times 9$ Latin square based on the $j^{\text {th }}$ symbol set. Figure 6.15 is the 2-MOLS(9) based on the first symbol set.

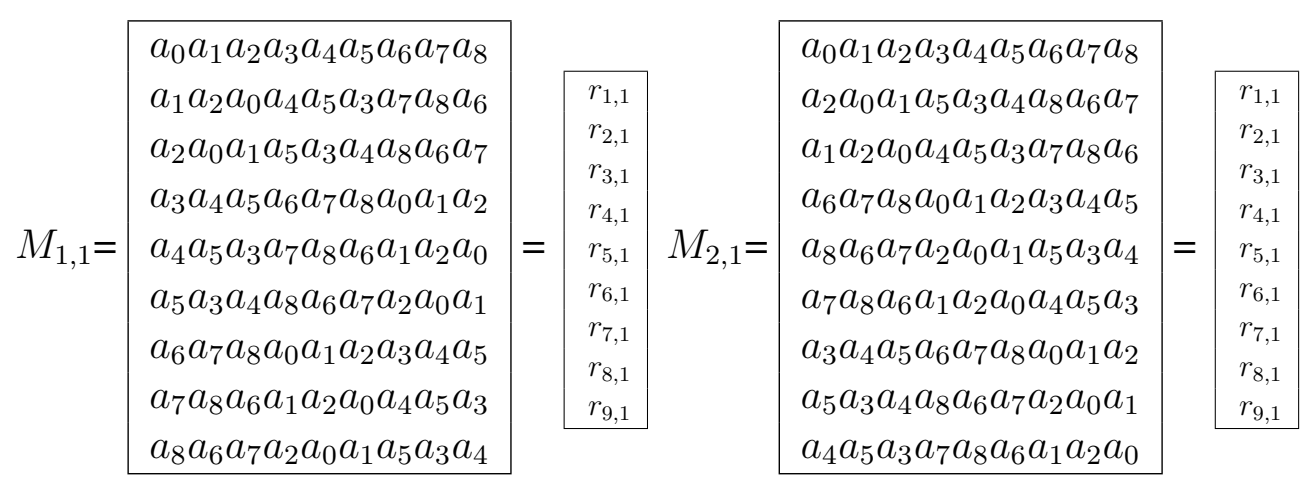

Figure 6.15: Converted $9 \times 9$ Latin square 
Now we create a 2-MOELR $(9,36 ; 18)$ using Lemma 6.14 with 2-MOLS $(9)$ and the first two rows of an $\mathrm{OA}(3,2)$. Here $k=2, n_{1}=2, n_{2}=9$.

$$
\begin{aligned}
& \mathcal{R}_{1}=\begin{array}{|l|l|l|lll|}
M_{1,1} & M_{1,1} & M_{1,2} & M_{1,2} \\
\hline r_{1,1} & r_{1,1} & r_{1,2} & r_{1,2} \\
r_{2,1} & r_{2,1} & r_{2,2} & r_{2,2} \\
r_{3,1} & r_{3,1} & r_{3,2} & r_{3,2} \\
r_{4,1} & r_{4,1} & r_{4,2} & r_{4,2} \\
r_{5,1} & r_{5,1} & r_{5,2} & r_{5,2} \\
r_{6,1} & r_{6,1} & r_{6,2} & r_{6,2} \\
r_{7,1} & r_{7,1} & r_{7,2} & r_{7,2} \\
r_{8,1} & r_{8,1} & r_{8,2} & r_{8,2} \\
r_{9,1} & r_{9,1} & r_{9,2} & r_{9,2} \\
\hline
\end{array}
\end{aligned}
$$

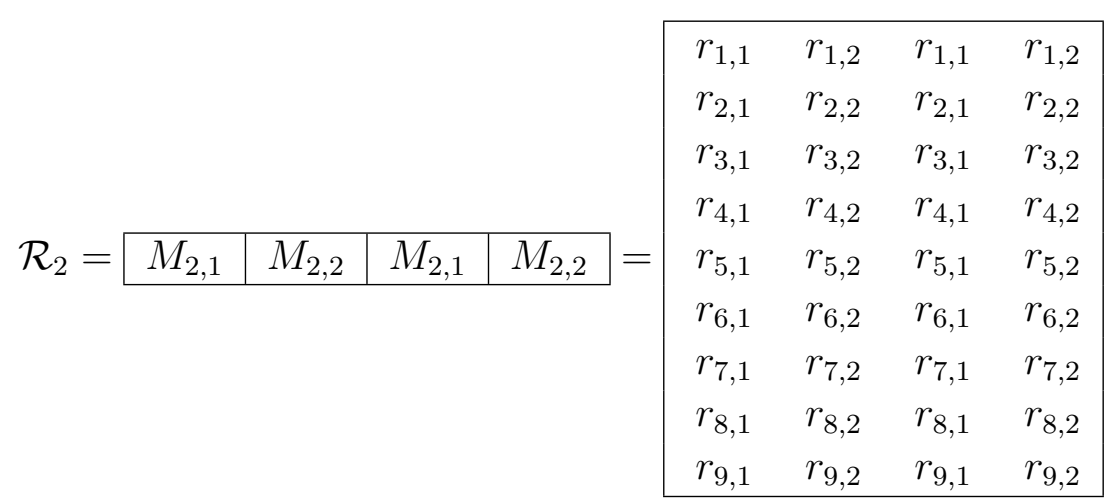

We now apply Lemma 6.16 with $m=2, s=9, a=4$, and $b=81$. It follows that from $\mathcal{R}_{1}$, we get

$$
\vec{v}=\left(r_{1,1}, r_{2,1}, r_{3,1}, \ldots, r_{9,1}, r_{1,1}, \ldots, r_{9,1}, r_{1,2}, \ldots, r_{9,2}, r_{1,2}, \ldots, r_{9,2}\right),
$$

and

$$
\begin{aligned}
& \vec{v}_{0}^{T}=\vec{v}[1,2,3,4]^{T}=\left(r_{1,1}, r_{2,1}, r_{3,1}, r_{4,1}\right)^{T} \\
& \vec{v}_{1}^{T}=\vec{v}[5,6,7,8]^{T}=\left(r_{5,1}, r_{6,1}, r_{7,1}, r_{8,1}\right)^{T} \\
& \vec{v}_{2}^{T}=\vec{v}[9,10,11,12]^{T}=\left(r_{9,1}, r_{1,1}, r_{2,1}, r_{3,1}\right)^{T} \\
& \vec{v}_{3}^{T}=\vec{v}[13,14,15,16]^{T}=\left(r_{4,1}, r_{5,1}, r_{6,1}, r_{7,1}\right)^{T} \\
& \vec{v}_{4}^{T}=\vec{v}[17,18,19,20]^{T}=\left(r_{8,1}, r_{9,1}, r_{1,2}, r_{2,2}\right)^{T} \\
& \vec{v}_{5}^{T}=\vec{v}[21,22,23,24]^{T}=\left(r_{3,2}, r_{4,2}, r_{5,2}, r_{6,2}\right)^{T} \\
& \vec{v}_{6}^{T}=\vec{v}[25,26,27,28]^{T}=\left(r_{7,2}, r_{8,2}, r_{9,2}, r_{1,2}\right)^{T} \\
& \vec{v}_{7}^{T}=\vec{v}[29,30,31,32]^{T}=\left(r_{2,2}, r_{3,2}, r_{4,2}, r_{5,2}\right)^{T} \\
& \vec{v}_{8}^{T}=\vec{v}[33,34,35,36]^{T}=\left(r_{6,2}, r_{7,2}, r_{8,2}, r_{9,2}\right)^{T}
\end{aligned}
$$

So we get $\mathcal{R}_{1}^{\prime \prime}=\left(\vec{v}_{0}^{T}, \vec{v}_{1}^{T}, \vec{v}_{2}^{T}, \vec{v}_{3}^{T}, \vec{v}_{4}^{T}\right)$. Thus the $2-\operatorname{MOELR}(4,81 ; 18)$ is given below. 


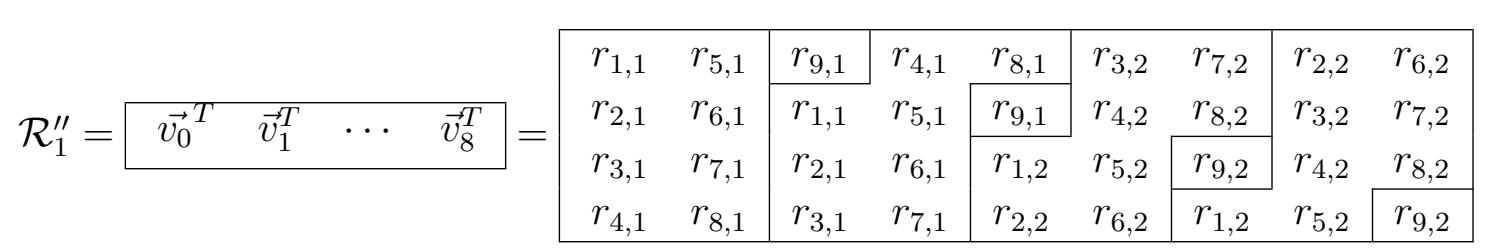

and

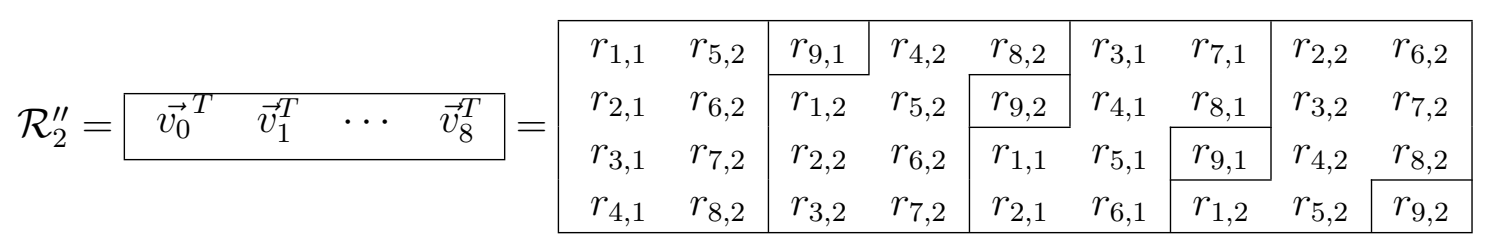

Lemma 6.20. If $t>2$, and there exists $y_{1}-\operatorname{MOLS}(t)$ and $a y_{2}-\operatorname{MOELR}\left(\frac{a}{t}, \frac{b}{t} ; \frac{n}{t}\right)$, then there exists a

$$
\left(\min \left\{y_{1}, y_{2}\right\}\right)-\operatorname{MOELR}(a, b ; n) .
$$

Proof: Let $m=\min \left\{y_{1}, y_{2}\right\}$ and let $A_{1}, A_{2}, \ldots, A_{m}$ be $m-\operatorname{MOLS}(t)$. For $j=1,2, \ldots, t$, define $S_{j}$ to be a set of $\frac{n}{t}$ symbols, so that $\bigcup_{j} S_{j}$ is a partition of a set of $n$ distinct symbols. Define $B_{i, j}$ to be the $i^{\text {th }}$ rectangle of an $m-\operatorname{MOELR}\left(\frac{a}{t}, \frac{b}{t} ; \frac{n}{t}\right)$ on symbol set $S_{j}$. Now replace each entry $j \in A_{i}$ with $B_{i, j}$. This forms a set of $m(a \times b)$-rectangles, $M_{1}, M_{2}, \ldots, M_{m}$, which we now prove is an $m-\operatorname{MOELR}(a, b ; n)$.

For any pair of rectangles $M_{r}$ and $M_{s}$, we had that $A_{r}$ and $A_{s}$ were orthogonal, so every pair of symbol sets $\left(S_{j_{1}}, S_{j_{2}}\right)$ has occurred exactly once among the $t^{2}$ pairs, $1 \leq j_{1}, j_{2} \leq t$. Furthermore, $B_{r, j_{1}}$ and $B_{s, j_{2}}$ are also orthogonal, so every ordered pair of symbols among the $\left(\frac{n}{t}\right)^{2}$ pairs has occurred exactly once. Thus, $M_{r}$ and $M_{s}$ are orthogonal.

Now consider any rectangle $M_{s}$. In each row, every symbol set $S_{j}$ for $j=1,2, \ldots, t$ occurs exactly once because $A_{s}$ is a Latin square. Also, every entry in symbol set $S_{j}$ occurs an equitable number of times in $B_{s, j}$, so each row of $M_{s}$ is equitable. The same argument holds for each column of $M_{s}$. So $M_{1}, M_{2}, \ldots, M_{m}$ forms an $m-\operatorname{MOELR}(a, b ; n)$.

Example 6.21. 2-MOELR $(12,27 ; 18)$ using Lemma 6.20

Note that $3 \mid 12$ and $3 \mid 27$ and there exists $3-\operatorname{MOELR}(4,9 ; 6)$. Let $B_{i, j}$ be the $i^{\text {th }}$ rectangle based on the $j^{\text {th }}$ symbol set where $i=1,2,3$ and $j=1,2,3$. For example,

$$
\begin{aligned}
& B_{1,1}=\begin{array}{lllllllll|}
1 & 2 & 3 & 4 & 5 & 6 & 3 & 5 & 2 \\
3 & 5 & 6 & 2 & 1 & 2 & 4 & 6 & 4 \\
4 & 6 & 1 & 5 & 3 & 1 & 2 & 4 & 3 \\
2 & 1 & 4 & 1 & 6 & 5 & 6 & 3 & 5
\end{array} \\
& B_{2,1}=\begin{array}{lllllllll|}
1 & 2 & 3 & 4 & 5 & 6 & 4 & 2 & 1 \\
2 & 6 & 1 & 5 & 3 & 4 & 1 & 5 & 2 \\
5 & 3 & 4 & 1 & 6 & 2 & 6 & 3 & 5 \\
3 & 5 & 6 & 6 & 4 & 3 & 2 & 1 & 4
\end{array}
\end{aligned}
$$




$B_{3,1}=\begin{array}{lllllllll}1 & 2 & 3 & 4 & 5 & 6 & 2 & 6 & 3 \\ 4 & 3 & 4 & 1 & 2 & 5 & 6 & 2 & 5 \\ 3 & 5 & 6 & 2 & 1 & 3 & 4 & 1 & 6 \\ 6 & 4 & 2 & 5 & 3 & 4 & 1 & 5 & 1\end{array}$.

Now using the structure of 2-MOLS(3) we get the following two matrices.

$$
M_{1}=\begin{array}{lll}
B_{1,1} & B_{1,2} & B_{1,3} \\
B_{1,2} & B_{1,3} & B_{1,1} \\
B_{1,3} & B_{1,1} & B_{1,2}
\end{array} \quad M_{2}=\begin{array}{lll}
B_{2,1} & B_{2,2} & B_{2,3} \\
B_{2,3} & B_{2,1} & B_{2,2} \\
B_{2,2} & B_{2,3} & B_{2,1} \\
\hline
\end{array}
$$

Thus, since orthogonality and equitability hold for $B_{i, j}$, and there exists 2-MOLS (3), we have a $2-\operatorname{MOELR}(12,27 ; 18)$.

Lemma 6.22. Suppose $a \leq b, n=h k$, and $a b=n^{2}$. Let

$$
x=\min \left\{N_{\operatorname{MOLS}}(h), N_{\mathrm{MOLS}}(k)\right\} .
$$

If $h \mid a$, then there exists an $x-\operatorname{MOELR}(a, b ; n)$.

Proof: Let $A_{1}, A_{2}, \ldots, A_{x}$ be $x-\operatorname{MOLS}(k)$. For $j=1,2, \ldots, k$, define $S_{j}$ to be a set of $h$ symbols, so that $\bigcup_{j=1}^{k} S_{j}$ is a partition of a set of $n$ distinct symbols. Define $B_{i, j}$ to be the $i^{\text {th }}$ Latin square of a set of $x-\operatorname{MOLS}(h)$ on symbol set $S_{j}$. Now replace each entry $j \in A_{i}$ with $B_{i, j}$. This forms a set of $x-\operatorname{MOLS}(n)$.

$A_{i}=$\begin{tabular}{|c|c|c|c|}
\hline 1 & 2 & $\cdots$ & $k$ \\
\hline$\vdots$ & $\vdots$ & $\vdots$ & $\vdots$
\end{tabular}$\rightarrow$\begin{tabular}{|c|c|c|c|}
\hline$B_{i, 1}$ & $B_{i, 2}$ & $\cdots$ & $B_{i, k}$ \\
\hline$\vdots$ & $\vdots$ & $\vdots$ & $\vdots$ \\
\hline
\end{tabular}

Permute the columns of each of these Latin squares according to the following permutation. For $m=0,1, \ldots, k-1$, let $m h+u \mapsto(u-1) k+(m+1)$ for $u=1, \ldots, h$. This permutation creates $h \times k$ sub-squares which has each of the first columns of the $B_{i, j}$ subsquares, followed by each second column and so on. Because there were $k$ sub-squares in each row, we can now consider each Latin square being composed of $h \times k$ sub-squares $H_{j}$, $j=1, \ldots, h$, in which each column contains entries from a different symbol set. Because there are $k$ symbol sets, every row of the $H_{j}$ contains exactly one entry from each symbol set as in Figure 6.16.

\begin{tabular}{c|c|c|c}
$1^{s t}$ columns & $2^{n d}$ columns & $\cdots$ & $c^{\text {th }}$ columns \\
\hline$H_{1}$ & $H_{2}$ & $\cdots$ & $H_{h}$ \\
$H_{h+1}$ & $H_{h+2}$ & $\cdots$ & $H_{2 h}$ \\
$\vdots$ & $\vdots$ & $\ddots$ & $\vdots$ \\
$H_{(k-1) h+1}$ & $H_{(k-1) h+2}$ & $\cdots$ & $H_{k h}$
\end{tabular}

Figure 6.16: Representation of the first mutually orthogonal Latin square of the $n \times n$ matrix. 
This matrix has $n=h k$ sub-squares. Because $h \mid a$, we can write $a=h r$ for some positive integer $r$. Arrange the sub-squares into an $r \times c$ matrix $L_{i}$ as follows.

$L_{i}=$\begin{tabular}{|c|c|c|c|}
\hline$H_{1}$ & $H_{2}$ & $\cdots$ & $H_{c}$ \\
\hline$H_{c+1}$ & $H_{c+2}$ & $\cdots$ & $H_{2 c}$ \\
\hline$\vdots$ & $\vdots$ & $\ddots$ & $\vdots$ \\
\hline$H_{(r-1) c+1}$ & $H_{(r-1) c+2}$ & $\cdots$ & $H_{r c}$ \\
\hline
\end{tabular}

Notice that $L_{i}$ has $c=\frac{h k}{r}$ sub-squares in each row, and it has $r$ sub-squares in each column. Therefore, $L_{i}$ has $h r=a$ rows and $c k$ columns. Because $n^{2}=h^{2} k^{2}=a b$, it follows that

$$
b=\frac{h^{2} k^{2}}{a}=\frac{k h k}{r}=k\left(\frac{h k}{r}\right)=k c .
$$

Thus $L_{i}$ has $b$ columns.

We now show that $\left\{L_{i}: i=1,2, \ldots, x\right\}$ forms a $x-\operatorname{MOELR}(a, b ; n)$. Because we construct $L_{i}$ in the same way for each $L_{i}$ orthogonality holds. Because every row of $H_{j}$ contains exactly 1 entry from each of the $k$ symbol sets, it follows that every symbol occurs exactly $\left\lfloor\frac{c}{h}\right\rfloor$ or $\left\lceil\frac{c}{h}\right\rceil$ times in every row of $L_{i}$. Because $c k=b$, it follows that $\frac{c}{h}=\frac{b}{h k}=\frac{b}{n}$. Therefore each symbol occurs $\left\lfloor\frac{b}{n}\right\rfloor$ times or $\left\lceil\frac{b}{n}\right\rceil$ times in each row of $L_{i}$.

Now suppose there is a column in $L_{i}$ that is not equitable. Then there would have to be a repeated entry in this column. This would mean we had some column in $A_{i}$ with a repeated entry. But $A_{i}$ was an $\operatorname{LS}(k)$, so that could not have happened. Therefore, each column is equitable. This shows that we have $x-\operatorname{MOELR}(a, b ; n)$.

Example 6.23. A 2-MOELR $(16,25 ; 20)$ using Lemma 6.22

Let $h=4$ and $k=5$. In Figure 6.17, we give 2-MOLS(20) by using 2-MOLS (4) and 2-MOLS (5).

Permute the columns according to the given permutation. Note that the first 5 columns were the $1^{\text {st }}$ columns of each $4 \times 4$ subsquare; the next 5 columns were the $2^{\text {nd }}$ column of each $4 \times 4$ subsquare, and so on. The permuted matrices are given in Figure 6.18

The 2-MOELR $(16,25 ; 20)$ is given in Figure 6.19.

\subsection{Main Theorem}

Combining the results given in (Chowla, Erdös, and Straus [15]) and (Colbourn, Dinitz, [18]), we have that $N_{\text {MOLS }}(n) \geq 3$ for $n \geq 11$.

Lemma 6.24. If $n^{2}=a b$ and $\operatorname{gcd}(n, 6)=1$, then $N_{\text {MOELR }}(a, b ; n) \geq 3$.

Proof: Without loss, assume $a \leq b$. If $n$ is a prime power, then apply Corollary 6.13. Otherwise $n$ is a product of prime powers not involving 2 or 3 . Let $h=p$ be a prime such 


\begin{tabular}{|cccc|cccc|cccc|cccc|cccc|}
\hline 1 & 2 & 3 & 4 & 5 & 6 & 7 & 8 & 9 & 10 & 11 & 12 & 13 & 14 & 15 & 16 & 17 & 18 & 19 & 20 \\
2 & 1 & 4 & 3 & 6 & 5 & 8 & 7 & 10 & 9 & 12 & 11 & 14 & 13 & 16 & 15 & 18 & 17 & 20 & 19 \\
3 & 4 & 1 & 2 & 7 & 8 & 5 & 6 & 11 & 12 & 9 & 10 & 15 & 16 & 13 & 14 & 19 & 20 & 17 & 18 \\
4 & 3 & 2 & 1 & 8 & 7 & 6 & 5 & 12 & 11 & 10 & 9 & 16 & 15 & 14 & 13 & 20 & 19 & 18 & 17 \\
\hline 17 & 18 & 19 & 20 & 1 & 2 & 3 & 4 & 5 & 6 & 7 & 8 & 9 & 10 & 11 & 12 & 13 & 14 & 15 & 16 \\
18 & 17 & 20 & 19 & 2 & 1 & 4 & 3 & 6 & 5 & 8 & 7 & 10 & 9 & 12 & 11 & 14 & 13 & 16 & 15 \\
19 & 20 & 17 & 18 & 3 & 4 & 1 & 2 & 7 & 8 & 5 & 6 & 11 & 12 & 9 & 10 & 15 & 16 & 13 & 14 \\
20 & 19 & 18 & 17 & 4 & 3 & 2 & 1 & 8 & 7 & 6 & 5 & 12 & 11 & 10 & 9 & 16 & 15 & 14 & 13 \\
\hline 13 & 14 & 15 & 16 & 17 & 18 & 19 & 20 & 1 & 2 & 3 & 4 & 5 & 6 & 7 & 8 & 9 & 10 & 11 & 12 \\
14 & 13 & 16 & 15 & 18 & 17 & 20 & 19 & 2 & 1 & 4 & 3 & 6 & 5 & 8 & 7 & 10 & 9 & 12 & 11 \\
15 & 16 & 13 & 14 & 19 & 20 & 17 & 18 & 3 & 4 & 1 & 2 & 7 & 8 & 5 & 6 & 11 & 12 & 9 & 10 \\
16 & 15 & 14 & 13 & 20 & 19 & 18 & 17 & 4 & 3 & 2 & 1 & 8 & 7 & 6 & 5 & 12 & 11 & 10 & 9 \\
\hline 9 & 10 & 11 & 12 & 13 & 14 & 15 & 16 & 17 & 18 & 19 & 20 & 1 & 2 & 3 & 4 & 5 & 6 & 7 & 8 \\
10 & 9 & 12 & 11 & 14 & 13 & 16 & 15 & 18 & 17 & 20 & 19 & 2 & 1 & 4 & 3 & 6 & 5 & 8 & 7 \\
11 & 12 & 9 & 10 & 15 & 16 & 13 & 14 & 19 & 20 & 17 & 18 & 3 & 4 & 1 & 2 & 7 & 8 & 5 & 6 \\
12 & 11 & 10 & 9 & 16 & 15 & 14 & 13 & 20 & 19 & 18 & 17 & 4 & 3 & 2 & 1 & 8 & 7 & 6 & 5 \\
\hline 5 & 6 & 7 & 8 & 9 & 10 & 11 & 12 & 13 & 14 & 15 & 16 & 17 & 18 & 19 & 20 & 1 & 2 & 3 & 4 \\
6 & 5 & 8 & 7 & 10 & 9 & 12 & 11 & 14 & 13 & 16 & 15 & 18 & 17 & 20 & 19 & 2 & 1 & 4 & 3 \\
7 & 8 & 5 & 6 & 11 & 12 & 9 & 10 & 15 & 16 & 13 & 14 & 19 & 20 & 17 & 18 & 3 & 4 & 1 & 2 \\
8 & 7 & 6 & 5 & 12 & 11 & 10 & 9 & 16 & 15 & 14 & 13 & 20 & 19 & 18 & 17 & 4 & 3 & 2 & 1 \\
\hline
\end{tabular}

\begin{tabular}{|cccc|cccc|cccc|cccc|cccc|}
\hline 1 & 2 & 3 & 4 & 5 & 6 & 7 & 8 & 9 & 10 & 11 & 12 & 13 & 14 & 15 & 16 & 17 & 18 & 19 & 20 \\
3 & 4 & 1 & 2 & 7 & 8 & 5 & 6 & 11 & 12 & 9 & 10 & 15 & 16 & 13 & 14 & 19 & 20 & 17 & 18 \\
4 & 3 & 2 & 1 & 8 & 7 & 6 & 5 & 12 & 11 & 10 & 9 & 16 & 15 & 14 & 13 & 20 & 19 & 18 & 17 \\
2 & 1 & 4 & 3 & 6 & 5 & 8 & 7 & 10 & 9 & 12 & 11 & 14 & 13 & 16 & 15 & 18 & 17 & 20 & 19 \\
\hline 9 & 10 & 11 & 12 & 13 & 14 & 15 & 16 & 17 & 18 & 19 & 20 & 1 & 2 & 3 & 4 & 5 & 6 & 7 & 8 \\
11 & 12 & 9 & 10 & 15 & 16 & 13 & 14 & 19 & 20 & 17 & 18 & 3 & 4 & 1 & 2 & 7 & 8 & 5 & 6 \\
12 & 11 & 10 & 9 & 16 & 15 & 14 & 13 & 20 & 19 & 18 & 17 & 4 & 3 & 2 & 1 & 8 & 7 & 6 & 5 \\
10 & 9 & 12 & 11 & 14 & 13 & 16 & 15 & 18 & 17 & 20 & 19 & 2 & 1 & 4 & 3 & 6 & 5 & 8 & 7 \\
\hline 17 & 18 & 19 & 20 & 1 & 2 & 3 & 4 & 5 & 6 & 7 & 8 & 9 & 10 & 11 & 12 & 13 & 14 & 15 & 16 \\
19 & 20 & 17 & 18 & 3 & 4 & 1 & 2 & 7 & 8 & 5 & 6 & 11 & 12 & 9 & 10 & 15 & 16 & 13 & 14 \\
20 & 19 & 18 & 17 & 4 & 3 & 2 & 1 & 8 & 7 & 6 & 5 & 12 & 11 & 10 & 9 & 16 & 15 & 14 & 13 \\
18 & 17 & 20 & 19 & 2 & 1 & 4 & 3 & 6 & 5 & 8 & 7 & 10 & 9 & 12 & 11 & 14 & 13 & 16 & 15 \\
\hline 5 & 6 & 7 & 8 & 9 & 10 & 11 & 12 & 13 & 14 & 15 & 16 & 17 & 18 & 19 & 20 & 1 & 2 & 3 & 4 \\
7 & 8 & 5 & 6 & 11 & 12 & 9 & 10 & 15 & 16 & 13 & 14 & 19 & 20 & 17 & 18 & 3 & 4 & 1 & 2 \\
8 & 7 & 6 & 5 & 12 & 11 & 10 & 9 & 16 & 15 & 14 & 13 & 20 & 19 & 18 & 17 & 4 & 3 & 2 & 1 \\
6 & 5 & 8 & 7 & 10 & 9 & 12 & 11 & 14 & 13 & 16 & 15 & 18 & 17 & 20 & 19 & 2 & 1 & 4 & 3 \\
\hline 13 & 14 & 15 & 16 & 17 & 18 & 19 & 20 & 1 & 2 & 3 & 4 & 5 & 6 & 7 & 8 & 9 & 10 & 11 & 12 \\
15 & 16 & 13 & 14 & 19 & 20 & 17 & 18 & 3 & 4 & 1 & 2 & 7 & 8 & 5 & 6 & 11 & 12 & 9 & 10 \\
16 & 15 & 14 & 13 & 20 & 19 & 18 & 17 & 4 & 3 & 2 & 1 & 8 & 7 & 6 & 5 & 12 & 11 & 10 & 9 \\
14 & 13 & 16 & 15 & 18 & 17 & 20 & 19 & 2 & 1 & 4 & 3 & 6 & 5 & 8 & 7 & 10 & 9 & 12 & 11 \\
\hline
\end{tabular}

Figure 6.17: A 2-MOLS(20)

that $p \mid a$ and $k=\frac{n}{p}$. Because $h>3$ is prime, it follows that $N_{\text {MOLS }}(h)>4$. We also have that $k \geq 35$ or $k$ is prime, so $N_{\text {MOLS }}(k) \geq 3$. Now apply Lemma 6.22.

Lemma 6.25. If $n^{2}=a b$ and $\operatorname{gcd}(n, 3)=1$, then $N_{\text {MOELR }}(a, b ; n) \geq 3$, except possibly when $(a, b ; n) \in\{(2,50 ; 10),(49,100 ; 70)\}$.

Proof: If $n$ is odd then we apply Lemma 6.24. Otherwise let $n$ be even and without loss assume that $a \leq b$.

If $a$ has an odd prime divisor $h$, then $h>3$ and $N_{\text {MOLS }}(h) \geq 3$. Also, $h$ is a divisor of $n$ and $k=\frac{n}{h}$ is such that $N_{\text {MOLS }}(k) \geq 3$ unless $k=2$ or 10 . Thus if $k \neq 2,10$ we may apply Lemma 6.22 to obtain a 3-MOELR $(n)$. If $k=2$, then $n=2 p$ for an odd prime $p>3$. Then there are 2 possibilities for $a ; a=p$ or $2 p$. In each case $a \mid n$ and we can apply Lemma 6.11. If $k=10$, then $n=10 p$ for an odd prime $p>3$. Then there are 5 possibilities for $a$. If $a=p, 2 p, 5 p$, or $2 p$, then $a \mid n$ and we can apply Lemma 6.11. If $a=p^{2}$, then $b=100$ and thus because $a<b, p=5$ or $p=7$. When $p=5$ we may use 


\begin{tabular}{|ccccc|ccccc|ccccc|ccccc|}
\hline 1 & 5 & 9 & 13 & 17 & 2 & 6 & 10 & 14 & 18 & 3 & 7 & 11 & 15 & 19 & 4 & 8 & 12 & 16 & 20 \\
2 & 6 & 10 & 14 & 18 & 1 & 5 & 9 & 13 & 17 & 4 & 8 & 12 & 16 & 20 & 3 & 7 & 11 & 15 & 19 \\
3 & 7 & 11 & 15 & 19 & 4 & 8 & 12 & 16 & 20 & 1 & 5 & 9 & 13 & 17 & 2 & 6 & 10 & 14 & 18 \\
4 & 8 & 12 & 16 & 20 & 3 & 7 & 11 & 15 & 19 & 2 & 6 & 10 & 14 & 18 & 1 & 5 & 9 & 13 & 17 \\
\hline 17 & 1 & 5 & 9 & 13 & 18 & 2 & 6 & 10 & 14 & 19 & 3 & 7 & 11 & 15 & 20 & 4 & 8 & 12 & 16 \\
18 & 2 & 6 & 10 & 14 & 17 & 1 & 5 & 9 & 13 & 20 & 4 & 8 & 12 & 16 & 19 & 3 & 7 & 11 & 15 \\
19 & 3 & 7 & 11 & 15 & 20 & 4 & 8 & 12 & 16 & 17 & 1 & 5 & 9 & 13 & 18 & 2 & 6 & 10 & 14 \\
20 & 4 & 8 & 12 & 16 & 19 & 3 & 7 & 11 & 15 & 18 & 2 & 6 & 10 & 14 & 17 & 1 & 5 & 9 & 13 \\
\hline 13 & 17 & 1 & 5 & 9 & 14 & 18 & 2 & 6 & 10 & 15 & 19 & 3 & 7 & 11 & 16 & 20 & 4 & 8 & 12 \\
14 & 18 & 2 & 6 & 10 & 13 & 17 & 1 & 5 & 9 & 16 & 20 & 4 & 8 & 12 & 15 & 19 & 3 & 7 & 11 \\
15 & 19 & 3 & 7 & 11 & 16 & 20 & 4 & 8 & 12 & 13 & 17 & 1 & 5 & 9 & 14 & 18 & 2 & 6 & 10 \\
16 & 20 & 4 & 8 & 12 & 15 & 19 & 3 & 7 & 11 & 14 & 18 & 2 & 6 & 10 & 13 & 17 & 1 & 5 & 9 \\
\hline 9 & 13 & 17 & 1 & 5 & 10 & 14 & 18 & 2 & 6 & 11 & 15 & 19 & 3 & 7 & 12 & 16 & 20 & 4 & 8 \\
10 & 14 & 18 & 2 & 6 & 9 & 13 & 17 & 1 & 5 & 12 & 16 & 20 & 4 & 8 & 11 & 15 & 19 & 3 & 7 \\
11 & 15 & 19 & 3 & 7 & 12 & 16 & 20 & 4 & 8 & 9 & 13 & 17 & 1 & 5 & 10 & 14 & 18 & 2 & 6 \\
12 & 16 & 20 & 4 & 8 & 11 & 15 & 19 & 3 & 7 & 10 & 14 & 18 & 2 & 6 & 9 & 13 & 17 & 1 & 5 \\
\hline 5 & 9 & 13 & 17 & 1 & 6 & 10 & 14 & 18 & 2 & 7 & 11 & 15 & 19 & 3 & 8 & 12 & 16 & 20 & 4 \\
6 & 10 & 14 & 18 & 2 & 5 & 9 & 13 & 17 & 1 & 8 & 12 & 16 & 20 & 4 & 7 & 11 & 15 & 19 & 3 \\
7 & 11 & 15 & 19 & 3 & 8 & 12 & 16 & 20 & 4 & 5 & 9 & 13 & 17 & 1 & 6 & 10 & 14 & 18 & 2 \\
8 & 12 & 16 & 20 & 4 & 7 & 11 & 15 & 19 & 3 & 6 & 10 & 14 & 18 & 2 & 5 & 9 & 13 & 17 & 1 \\
\hline
\end{tabular}

\begin{tabular}{|ccccc|ccccc|ccccc|ccccc|}
\hline 1 & 5 & 9 & 13 & 17 & 2 & 6 & 10 & 14 & 18 & 3 & 7 & 11 & 15 & 19 & 4 & 8 & 12 & 16 & 20 \\
3 & 7 & 11 & 15 & 19 & 4 & 8 & 12 & 16 & 20 & 1 & 5 & 9 & 13 & 17 & 2 & 6 & 10 & 14 & 18 \\
4 & 8 & 12 & 16 & 20 & 3 & 7 & 11 & 15 & 19 & 2 & 6 & 10 & 14 & 18 & 1 & 5 & 9 & 13 & 17 \\
2 & 6 & 10 & 14 & 18 & 1 & 5 & 9 & 13 & 17 & 4 & 8 & 12 & 16 & 20 & 3 & 7 & 11 & 15 & 19 \\
\hline 9 & 13 & 17 & 1 & 5 & 10 & 14 & 18 & 2 & 6 & 11 & 15 & 19 & 3 & 7 & 12 & 16 & 20 & 4 & 8 \\
11 & 15 & 19 & 3 & 7 & 12 & 16 & 20 & 4 & 8 & 9 & 13 & 17 & 1 & 5 & 10 & 14 & 18 & 2 & 6 \\
12 & 16 & 20 & 4 & 8 & 11 & 15 & 19 & 3 & 7 & 10 & 14 & 18 & 2 & 6 & 9 & 13 & 17 & 1 & 5 \\
10 & 14 & 18 & 2 & 6 & 9 & 13 & 17 & 1 & 5 & 12 & 16 & 20 & 4 & 8 & 11 & 15 & 19 & 3 & 7 \\
\hline 17 & 1 & 5 & 9 & 13 & 18 & 2 & 6 & 10 & 14 & 19 & 3 & 7 & 11 & 15 & 20 & 4 & 8 & 12 & 16 \\
19 & 3 & 7 & 11 & 15 & 20 & 4 & 8 & 12 & 16 & 17 & 1 & 5 & 9 & 13 & 18 & 2 & 6 & 10 & 14 \\
20 & 4 & 8 & 12 & 16 & 19 & 3 & 7 & 11 & 15 & 18 & 2 & 6 & 10 & 14 & 17 & 1 & 5 & 9 & 13 \\
18 & 2 & 6 & 10 & 14 & 17 & 1 & 5 & 9 & 13 & 20 & 4 & 8 & 12 & 16 & 19 & 3 & 7 & 11 & 15 \\
\hline 5 & 9 & 13 & 17 & 1 & 6 & 10 & 14 & 18 & 2 & 7 & 11 & 15 & 19 & 3 & 8 & 12 & 16 & 20 & 4 \\
7 & 11 & 15 & 19 & 3 & 8 & 12 & 16 & 20 & 4 & 5 & 9 & 13 & 17 & 1 & 6 & 10 & 14 & 18 & 2 \\
8 & 12 & 16 & 20 & 4 & 7 & 11 & 15 & 19 & 3 & 6 & 10 & 14 & 18 & 2 & 5 & 9 & 13 & 17 & 1 \\
6 & 10 & 14 & 18 & 2 & 5 & 9 & 13 & 17 & 1 & 8 & 12 & 16 & 20 & 4 & 7 & 11 & 15 & 19 & 3 \\
\hline 13 & 17 & 1 & 5 & 9 & 14 & 18 & 2 & 6 & 10 & 15 & 19 & 3 & 7 & 11 & 16 & 20 & 4 & 8 & 12 \\
15 & 19 & 3 & 7 & 11 & 16 & 20 & 4 & 8 & 12 & 13 & 17 & 1 & 5 & 9 & 14 & 18 & 2 & 6 & 10 \\
16 & 20 & 4 & 8 & 12 & 15 & 19 & 3 & 7 & 11 & 14 & 18 & 2 & 6 & 10 & 13 & 17 & 1 & 5 & 9 \\
14 & 18 & 2 & 6 & 10 & 13 & 17 & 1 & 5 & 9 & 16 & 20 & 4 & 8 & 12 & 15 & 19 & 3 & 7 & 11 \\
\hline
\end{tabular}

Figure 6.18: Permuted matrices of a 2-MOLS $(20)$.

Lemma 6.11, however when $p=7$ we have the exception $(a, b ; n)=(49,100 ; 70)$ to the theorem.

Now suppose $a=2^{\ell}$ for some $\ell \geq 1$.

If $\ell=1$, then $a=2$ and divides $n$ so we may apply Lemma 6.11, unless $n=10$ where it is not known if $3-$ MOLS of order 10 exist. This is the exception $(a, b ; n)=(2,50 ; 10)$ to the theorem.

If $\ell>2$, then we are guaranteed that $4 \mid n$. However, if $\ell=2$, then it is possible that $4 \nmid n$. In this case, $n=2 s$ where $s$ is a product of prime powers not involving 2 or 3 . There exists an $\mathrm{OA}(3,2)$ and 3-MOLS $(s)$. So let $m=2$ and apply Lemma 6.16 to obtain a $3-\operatorname{MOELR}(a, b ; n)$ for all $s>8$. If $s=5$, then $n=10$ and a $3-\operatorname{MOELR}(4,25 ; 10)$ was constructed in Example 6.7. If $s=7$, then $n=14$ and there exists a $3-\operatorname{MOELR}(4,49 ; 14)$ by Example 6.8. If $s=8$, then $n=16$ and is a prime power, so apply Corollary 6.11.

Lemma 6.26. If $n^{2}=a b$ and $n$ is odd, then $N_{\operatorname{MOELR}}(a, b ; n) \geq 3$, except possibly when $(a, b ; n) \in\{(9,25 ; 15),(9,49 ; 21)\}$ or $a=9$ and $n=3$ s where $s \equiv 5,7,11$, or 13 


$M_{1}=$\begin{tabular}{|ccccc|ccccc|cccccc|ccccc|ccccc|}
\hline 1 & 5 & 9 & 13 & 17 & 2 & 6 & 10 & 14 & 18 & 3 & 7 & 11 & 15 & 19 & 4 & 8 & 12 & 16 & 20 & 17 & 1 & 5 & 9 & 13 \\
2 & 6 & 10 & 14 & 18 & 1 & 5 & 9 & 13 & 17 & 4 & 8 & 12 & 16 & 20 & 3 & 7 & 11 & 15 & 19 & 18 & 2 & 6 & 10 & 14 \\
3 & 7 & 11 & 15 & 19 & 4 & 8 & 12 & 16 & 20 & 1 & 5 & 9 & 13 & 17 & 2 & 6 & 10 & 14 & 18 & 19 & 3 & 7 & 11 & 15 \\
4 & 8 & 12 & 16 & 20 & 3 & 7 & 11 & 15 & 19 & 2 & 6 & 10 & 14 & 18 & 1 & 5 & 9 & 13 & 17 & 20 & 4 & 8 & 12 & 16 \\
\hline 18 & 2 & 6 & 10 & 14 & 19 & 3 & 7 & 11 & 15 & 20 & 4 & 8 & 12 & 16 & 13 & 17 & 1 & 5 & 9 & 14 & 18 & 2 & 6 & 10 \\
17 & 1 & 5 & 9 & 13 & 20 & 4 & 8 & 12 & 16 & 19 & 3 & 7 & 11 & 15 & 14 & 18 & 2 & 6 & 10 & 13 & 17 & 1 & 5 & 9 \\
20 & 4 & 8 & 12 & 16 & 17 & 1 & 5 & 9 & 13 & 18 & 2 & 6 & 10 & 14 & 15 & 19 & 3 & 7 & 11 & 16 & 20 & 4 & 8 & 12 \\
19 & 3 & 7 & 11 & 15 & 18 & 2 & 6 & 10 & 14 & 17 & 1 & 5 & 9 & 13 & 16 & 20 & 4 & 8 & 12 & 15 & 19 & 3 & 7 & 11 \\
\hline 15 & 19 & 3 & 7 & 11 & 16 & 20 & 4 & 8 & 12 & 9 & 13 & 17 & 1 & 5 & 10 & 14 & 18 & 2 & 6 & 11 & 15 & 19 & 3 & 7 \\
16 & 20 & 4 & 8 & 12 & 15 & 19 & 3 & 7 & 11 & 10 & 14 & 18 & 2 & 6 & 9 & 13 & 17 & 1 & 5 & 12 & 16 & 20 & 4 & 8 \\
13 & 17 & 1 & 5 & 9 & 14 & 18 & 2 & 6 & 10 & 11 & 15 & 19 & 3 & 7 & 12 & 16 & 20 & 4 & 8 & 9 & 13 & 17 & 1 & 5 \\
14 & 18 & 2 & 6 & 10 & 13 & 17 & 1 & 5 & 9 & 12 & 16 & 20 & 4 & 8 & 11 & 15 & 19 & 3 & 7 & 10 & 14 & 18 & 2 & 6 \\
\hline 12 & 16 & 20 & 4 & 8 & 5 & 9 & 13 & 17 & 1 & 6 & 10 & 14 & 18 & 2 & 7 & 11 & 15 & 19 & 3 & 8 & 12 & 16 & 20 & 4 \\
11 & 15 & 19 & 3 & 7 & 6 & 10 & 14 & 18 & 2 & 5 & 9 & 13 & 17 & 1 & 8 & 12 & 16 & 20 & 4 & 7 & 11 & 15 & 19 & 3 \\
10 & 14 & 18 & 2 & 6 & 7 & 11 & 15 & 19 & 3 & 8 & 12 & 16 & 20 & 4 & 5 & 9 & 13 & 17 & 1 & 6 & 10 & 14 & 18 & 2 \\
9 & 13 & 17 & 1 & 5 & 8 & 12 & 16 & 20 & 4 & 7 & 11 & 15 & 19 & 3 & 6 & 10 & 14 & 18 & 2 & 5 & 9 & 13 & 17 & 1 \\
\hline
\end{tabular}

$M_{2}=$\begin{tabular}{|ccccc|ccccc|cccccc|ccccc|ccccc|}
\hline 1 & 5 & 9 & 13 & 17 & 3 & 7 & 11 & 15 & 19 & 4 & 8 & 12 & 16 & 20 & 2 & 6 & 10 & 14 & 18 & 9 & 13 & 17 & 1 & 5 \\
2 & 6 & 10 & 14 & 18 & 4 & 8 & 12 & 16 & 20 & 3 & 7 & 11 & 15 & 19 & 1 & 5 & 9 & 13 & 17 & 10 & 14 & 18 & 2 & 6 \\
3 & 7 & 11 & 15 & 19 & 1 & 5 & 9 & 13 & 17 & 2 & 6 & 10 & 14 & 18 & 4 & 8 & 12 & 16 & 20 & 11 & 15 & 19 & 3 & 7 \\
4 & 8 & 12 & 16 & 20 & 2 & 6 & 10 & 14 & 18 & 1 & 5 & 9 & 13 & 17 & 3 & 7 & 11 & 15 & 19 & 12 & 16 & 20 & 4 & 8 \\
\hline 11 & 15 & 19 & 3 & 7 & 12 & 16 & 20 & 4 & 8 & 10 & 14 & 18 & 2 & 6 & 17 & 1 & 5 & 9 & 13 & 19 & 3 & 7 & 11 & 15 \\
12 & 16 & 20 & 4 & 8 & 11 & 15 & 19 & 3 & 7 & 9 & 13 & 17 & 1 & 5 & 18 & 2 & 6 & 10 & 14 & 20 & 4 & 8 & 12 & 16 \\
9 & 13 & 17 & 1 & 5 & 10 & 14 & 18 & 2 & 6 & 12 & 16 & 20 & 4 & 8 & 19 & 3 & 7 & 11 & 15 & 17 & 1 & 5 & 9 & 13 \\
10 & 14 & 18 & 2 & 6 & 9 & 13 & 17 & 1 & 5 & 11 & 15 & 19 & 3 & 7 & 20 & 4 & 8 & 12 & 16 & 18 & 2 & 6 & 10 & 14 \\
\hline 20 & 4 & 8 & 12 & 16 & 18 & 2 & 6 & 10 & 14 & 5 & 9 & 13 & 17 & 1 & 7 & 11 & 15 & 19 & 3 & 8 & 12 & 16 & 20 & 4 \\
19 & 3 & 7 & 11 & 15 & 17 & 1 & 5 & 9 & 13 & 6 & 10 & 14 & 18 & 2 & 8 & 12 & 16 & 20 & 4 & 7 & 11 & 15 & 19 & 3 \\
18 & 2 & 6 & 10 & 14 & 20 & 4 & 8 & 12 & 16 & 7 & 11 & 15 & 19 & 3 & 5 & 9 & 13 & 17 & 1 & 6 & 10 & 14 & 18 & 2 \\
17 & 1 & 5 & 9 & 13 & 19 & 3 & 7 & 11 & 15 & 8 & 12 & 16 & 20 & 4 & 6 & 10 & 14 & 18 & 2 & 5 & 9 & 13 & 17 & 1 \\
\hline 6 & 10 & 14 & 18 & 2 & 13 & 17 & 1 & 5 & 9 & 15 & 19 & 3 & 7 & 11 & 16 & 20 & 4 & 8 & 12 & 14 & 18 & 2 & 6 & 10 \\
5 & 9 & 13 & 17 & 1 & 14 & 18 & 2 & 6 & 10 & 16 & 20 & 4 & 8 & 12 & 15 & 19 & 3 & 7 & 11 & 13 & 17 & 1 & 5 & 9 \\
8 & 12 & 16 & 20 & 4 & 15 & 19 & 3 & 7 & 11 & 13 & 17 & 1 & 5 & 9 & 14 & 18 & 2 & 6 & 10 & 16 & 20 & 4 & 8 & 12 \\
7 & 11 & 15 & 19 & 3 & 16 & 20 & 4 & 8 & 12 & 14 & 18 & 2 & 6 & 10 & 13 & 17 & 1 & 5 & 9 & 15 & 19 & 3 & 7 & 11 \\
\hline
\end{tabular}

Figure 6.19: A 2-MOELR $(16,25 ; 20)$

$(\bmod 18), s>9$.

Proof: If $\operatorname{gcd}(n, 3)=1$, then apply Lemma 6.25. Otherwise $3 \mid n$ and without loss assume that $a \leq b$. If $a$ has a prime divisor $h>3$, then $h$ is also a divisor of $n$ so write $n=h k$. Then $N_{\text {MOLS }}(h) \geq 3$ and $N_{\text {MOLS }}(k) \geq 3$ unless $k=3$. Thus, if $k \neq 3$, we may apply Lemma 6.22 to obtain a $3-\operatorname{MOELR}(a, b ; n)$. If $k=3$, then $n=3 p$ for a prime $p>3$. There are 2 possibilities for $a ; a=p$ or $3 p$. In each case $a \mid n$, so we can apply Lemma 6.11

Now suppose $a=3^{\ell}$ for some $\ell \geq 1$.

If $\ell=1$, then $a=3$ and divides $n$, so we may apply Lemma 6.11.

If $\ell \geq 2$ and $9 \mid n$, we set $h=9$ and $k=\frac{n}{9}$. Then $\min \left\{N_{\text {MOLS }}(h), N_{\text {MOLS }}(k)\right\} \geq 3$, unless $k=3$. So when $k \neq 3$ we may apply Lemma 6.22 to obtain $N_{\text {MOELR }}(a, b ; n) \geq 3$. If $k=3$, then $n=27$ and $a \in\{9,27\}$. In each case $a \mid n$, so apply Lemma 6.11.

If $\ell>2$, then we are guaranteed that $9 \mid n$. However, if $\ell=2$, then it is possible that $9 \nmid n$. In this case, $n=3 s$ where $s$ is a product of prime powers not involving 2 or 3 . There exists an $\mathrm{OA}(3,3)$ and 3-MOLS $(s)$. Let $m=3$ and apply Lemma 6.17 to obtain a $3-\operatorname{MOELR}(a, b ; n)$ for all $s>9$ and $s \equiv 1,8(\bmod 9)$. If $s=5$ or 7 , then $n=15$ or 21 and we have open cases $(a, b ; n) \in\{(9,25 ; 15),(9,49 ; 21)\}$. If $s>9$ and $s \equiv 2,4,5$, or 
$7(\bmod 9)$, then because $s \equiv 1$ or $5(\bmod 6)$, we have open cases when $s \equiv 5,7,11,13$ $(\bmod 18)$.

Lemma 6.27. If $n^{2}=a b$, then $N_{\text {MOELR }}(a, b ; n) \geq 3$ except possibly when $(a, b ; n) \in$ $\{(2,18 ; 6),(2,50 ; 10),(8,18 ; 12),(9,64 ; 24),(9,100 ; 30),(9,256 ; 48),(12,27 ; 18)$, $(18,32 ; 24),(18,50 ; 30),(18,98 ; 42),(18,128 ; 48),(27,300 ; 90),(36,49 ; 42),(36,121 ; 66)$, $(36,169 ; 78),(36,289 ; 102),(36,361 ; 114),(36,529 ; 138),(36,625 ; 150),(36,841 ; 174)$, $(36,961 ; 186),(36,1225 ; 210),(81,100 ; 90)\}$ or

$$
\begin{aligned}
& a=9 \text { and } n=3 s, \text { where } s \equiv 2,4,14,16(\bmod 18), s>9 \\
& a=18 \text { and } n=3 s, \text { where } s \equiv 4,8,10,14(\bmod 18), s>18, \\
& a=36 \text { and } n=6 s, \text { where } s \equiv 5,7,11,13,17,19,23,25,29,31(\bmod 36), s>36 .
\end{aligned}
$$

Proof: If $\operatorname{gcd}(n, 6)=1$, then apply Lemma 6.24. If $n$ is odd, then apply Lemma 6.26. If $n$ is even and $\operatorname{gcd}(n, 3)=1$, then apply Lemma 6.25. Otherwise $6 \mid n$, and without loss assume $a \leq b$.

If $a$ has a prime divisor $h>3$, then $h$ is also a divisor of $n$, so write $n=h k$. Then $N_{\text {MOLS }}(h) \geq 3$ and $N_{\text {MOLS }}(k) \geq 3$ unless $k=2,3,6,10$. Because $6 \mid n$, we cannot have $k=2,3$, or 10 . Thus, if $k \neq 6$, then we may apply Lemma 6.22 to obtain a $3-\operatorname{MOELR}(a, b ; n) \geq 3$.

If $k=6$, then $n=6 p$ for a prime $p>3$. There are 5 possibilities for $a$. If $a=p, 2 p, 3 p$, or $6 p$, then $a \mid n$ so apply Lemma 6.11. If $a=p^{2}$, then $b=36$, and thus because $a<b$, $p=5$, and we have the open case $(a, b ; n)=(25,36 ; 30)$.

Suppose $a=2^{\ell}$ for some $\ell \geq 1$.

If $\ell=1$, then $a=2$ and divides $n$, so we may apply Lemma 6.11 unless $n=6$ where $3-\operatorname{MOLS}(6)$ does not exist. This is the possible exception $(a, b ; n)=(2,18 ; 6)$ to the theorem.

If $\ell \geq 2$ and $4 \mid n$, then set $h=4$ and $k=\frac{n}{4}$. Hence $\min \left\{N_{\text {MOLS }}(h), N_{\text {MOLS }}(k)\right\} \geq 3$ unless $k=2,3,6$, or 10 . Thus when $k \neq 2,3,6$, or 10 we can apply Lemma 6.22 to obtain $N_{\text {MOELR }}(a, b ; n) \geq 3$. If $k=2$, then $n=8$ and $a=4$, so apply Lemma 6.11. If $k=3$, then $n=12$ and $a=4$ or 8 . If $a=4$, then apply Lemma 6.11. Otherwise, $a=8$ and we have the open case $(a, b ; n)=(8,18 ; 12)$. If $k=6$, then $n=24$ and $a \in\{4,8,16\}$. If $a=4$ or 8 then it divides $n$ so apply Lemma 6.11. If $a=16$, then $b=36$. Here we can apply Lemma 6.20 with $t=4$ because there exists a $3-\operatorname{MOELR}(4,9 ; 6)$. If $k=10$, then $n=40$ and $a \in\{4,8,16,32\}$. If $a=4$ or 8 , then it divides $n$ so apply Lemma 6.11. If $a=16$ or 32 , then $8 \mid a$ so we may apply Lemma 6.22 with $h=8$ and $k=5$ to obtain $N_{\text {MOELR }}(a, b ; n) \geq 3$.

If $\ell>2$, then we are guaranteed that $4 \mid n$. However if $\ell=2$, then it is possible that $4 \nmid n$. In this case, $n=2 s$ where $s$ is an odd product of prime powers involving 3. There exists an $\operatorname{OA}(3,2)$ and 3-MOLS $(s)$ if $s \neq 3$. So if $s \neq 3$, we can apply Lemma 6.16 with $m=2$ to obtain a 3-MOELR $(a, b ; n)$ for all $s>4$. If $s=3$, then $n=6$ and $3-\operatorname{MOELR}(4,9 ; 6)$ is given in Example 6.5 .

Suppose $a=3^{\ell}$ for some $\ell \geq 1$. 
If $\ell=1$, then $a=3$ and divides $n$, so apply Lemma 6.11 unless $n=6$ where $3-\operatorname{MOLS}(6)$ does not exist. In this case, a $3-\operatorname{MOELR}(3,12 ; 6)$ is given in Example 6.6.

If $\ell \geq 2$ and $9 \mid n$, then set $h=9$ and $k=\frac{n}{9}$. Then $\min \left\{N_{\text {MOLS }}(h), N_{\text {MOLS }}(k)\right\} \geq 3$ unless $k=2,3,6$, or 10 . Thus when $k \neq 2,3,6,10$, we may apply Lemma 6.22 to obtain $N_{\text {MOELR }}(a, b ; n) \geq 3$. If $k=2$, then $n=18$ and $a=9$ in which case $a \mid n$, so apply Lemma 6.11. If $k=3$, then $n=27$ and $a=9$ or 27 , so $a \mid n$ and we may apply Lemma 6.11. If $k=6$, then $n=54$ and $a=9$ or 27 , so $a \mid n$ and we may again apply Lemma 6.11. If $k=10$, then $n=90$ and $a=9,27$, or 81 . If $a=9$, then $a \mid n$ so apply Lemma 6.11 . Otherwise, we have two open cases: $(a, b ; n) \in\{(27,300 ; 90),(81,100 ; 90)\}$.

If $\ell>2$, then we are guaranteed that $9 \mid n$. However, if $\ell=2$, then it is possible that $9 \nmid n$. In this case, $n=3 s$ where $s$ is a product of even prime powers not involving 3. There exists an $\mathrm{OA}(3,3)$ and $3-\operatorname{MOLS}(s)$ if $s \neq 2$ or 10 and $s \equiv 1,8(\bmod 9)$. So if $s \neq 2$ or 10 and $s \equiv 1,8(\bmod 9)$, we may apply Lemma 6.17 with $m=3$ to obtain a $3-\operatorname{MOELR}(a, b ; n)$ for all $s>9$. If $(s, n)=(4,12)$, then we get the open case $(a, b ; n)=(9,16 ; 12)$. If $(s, n)=(2,6)$ then $a=9$ and $b=4$ so we need not consider this case. If $(s, n)=(6,18)$, then $(a, b ; n)=(9,36 ; 18)$. We may apply Lemma 6.20 with $t=3$ because a $3-\operatorname{MOELR}(3,12 ; 6)$ was given in Example 6.6. If $(s, n)=(8,24)$ we get the open case $(a, b ; n)=(9,64 ; 24)$, and if $(s, n)=(10,30)$, then we have the open case $(a, b ; n)=(9,100 ; 30)$. If $s>9$ and $s \equiv 2,4,5,7(\bmod 9)$, then because $s \equiv 2$ or 4 $(\bmod 6)$, we also have open cases when $s \equiv 2,4,14,16(\bmod 18)$.

Suppose $a=2^{\ell_{1}} 3^{\ell_{2}}$ for some $\ell_{1}, \ell_{2} \geq 1$.

If $\ell_{1}=\ell_{2}=1$, then $a=6$ and divides $n$ so we may apply Lemma 6.11 .

If $\ell_{1}=1$ and $\ell_{2} \geq 2$ and $18 \mid n$, we set $h=18$ and $k=\frac{n}{18}$. Then

$$
\min \left\{N_{\text {MOLS }}(h), N_{\text {MOLS }}(k)\right\} \geq 3
$$

unless $k=2,3,6$, or 10 . Thus if $k \neq 2,3,6$, or 10 we may apply Lemma 6.22 to obtain a $3-\operatorname{MOELR}(a, b ; n)$. If $k=2$, then $n=36$ and $a=18$. If $k=3$, then $n=54$ and $a \in\{18,54\}$. If $k=6$, then $n=108$ and $a \in\{18,54\}$. In each of these cases $a \mid n$, so apply Lemma 6.11. If $k=10$, then $n=180$ and $a \in\{18,54,162\}$. If $a=$ 18 , then $a \mid n$, so apply Lemma 6.11. If $a=54$ or 162 let $h=9$ and $k=20$. Then $\min \left(N_{\text {MOLS }}(h), N_{\text {MOLS }}(k)\right\} \geq 3$, so apply Lemma 6.22.

If $\ell_{1}=1$ and $\ell_{2}>2$, then we are guaranteed that 18|n. However, if $\ell_{1}=1$ and $\ell_{2}=2$, then it is possible that $18 \nmid n$. In this case $n=3 s$ where $s$ is an even product of prime powers not involving the prime 3. There exists an $\mathrm{OA}(3,3)$ and $3-\operatorname{MOLS}(s)$ if $s \neq 2$ or 10 and $s \equiv 2,16(\bmod 18)$. Let $m=3$ and apply Lemma 6.17 to obtain a $3-\operatorname{MOELR}(a, b ; n)$ for all $s>18$. If $(s, n) \in\{(2,6),(4,12)\}$, then $a>b$, so we need not consider these cases. If $(s, n) \in\{(8,24),(10,30),(14,42),(16,48)\}$, then we have the open cases $(a, b ; n) \in\{(18,32 ; 24),(18,50 ; 30),(18,98 ; 42),(18,128 ; 48)\}$. If $s>18$ and $s \equiv 4,8,10,14(\bmod 18)$, then we have open cases.

If $\ell_{2}=1$ and $\ell_{1} \geq 2$ and $12 \mid n$, we set $h=12$ and $k=\frac{n}{12}$. Then

$$
\min \left\{N_{\text {MOLS }}(h), N_{\text {MOLS }}(k)\right\} \geq 3
$$


unless $k=2,3,6$, or 10 . Thus if $k \neq 2,3,6$, or 10 , we may apply Lemma 6.22 to obtain a $3-\operatorname{MOELR}(a, b ; n)$. If $k=2$, then $n=24$ and $a=12$. Here $a \mid n$, so apply Lemma 6.11. If $k=3$, then $n=36$ and $a \in\{12,24\}$. If $a=12$, then it divides $n$, so apply Lemma 6.11. If $a=24$, then let $h=4$ and $k=9$ and apply Lemma 6.22. If $k=6$, then $n=72$ and $a \in\{12,24,48\}$. If $a=12$ or 24 , then it divides $n$, so apply Lemma 6.11. If $a=48$, then let $h=4$ and $k=18$ and apply Lemma 6.22. If $k=10$, then $n=120$ and $a \in\{12,24,48,96\}$. If $a=12$ or 24 , then it divides $n$ so apply Lemma 6.11. If $a=48$ or 96 , then let $h=4$ and $k=30$ and apply Lemma 6.22.

If $\ell_{2}=1$ and $\ell_{1}>2$, then we are guaranteed that $12 \mid n$. However, if $\ell_{2}=1$ and $\ell_{1}=2$, then it is possible that $12 \nmid n$. In this case, $n=2 s$ where $s$ is an odd product of prime powers involving the prime 3 . There exists an $\mathrm{OA}(3,2)$ and a set of $3-\operatorname{MOLS}(s)$ if $s \neq 3$. Let $m=2$ and apply Lemma 6.16 to obtain a $3-\operatorname{MOELR}(a, b ; n)$ for all $s>12$. If $(s, n)=(9,18)$, then we get the open case $(a, b ; n)=(12,27 ; 18)$. If $(s, n)=36$, then $a>b$ and we need not consider it.

If $\ell_{1} \geq 2$ and $\ell_{2} \geq 2$ and $36 \mid n$, then set $h=36$ and $k=\frac{n}{36}$. Then

$$
\min \left\{N_{\mathrm{MOLS}}(h), N_{\mathrm{MOLS}}(k)\right\} \geq 3
$$

unless $k=2,3,6$, or 10 . Thus, when $k \neq 2,3,6$, or 10 we can apply Lemma 6.22 to obtain $N_{\operatorname{MOELR}}(a, b ; n) \geq 3$. If $k=2$, then $n=72$ and $a=36$ or 72 . Here $a \mid n$ so apply Lemma 6.11. If $k=3$, then $n=108$ and $a=36,72$, or 108 . If $a=36$ or 108, then $a \mid n$ so apply Lemma 6.11. If $a=72$, then $9 \mid a$ so we may apply Lemma 6.22 with $h=9$ and $k=12$ to obtain $N_{\operatorname{MOELR}}(a, b ; n) \geq 3$. If $k=6$, then $n=216$ and $a \in\{36,72,108,144,216\}$. If $a \in\{36,72,108,216\}$, then $a \mid n$ and so apply Lemma 6.11. If $a=144$, then $12 \mid a$ so we may apply Lemma 6.22 with $h=12$ and $k=18$ to obtain $N_{\text {MOELR }}(a, b ; n) \geq 3$. If $k=10$, then $n=360$ and $a=\{36,72,108,144,216,288,324\}$. If $a=36$ or 72 , then $a \mid n$ so apply Lemma 6.11. Otherwise, we set $h=4$ and $k=90$ and apply Lemma 6.22 and apply Lemma 6.22 to obtain a $3-\operatorname{MOELR}(a, b ; n)$.

If $\ell_{1}>2$ and $\ell_{2}>2$, then we are guaranteed that $36 \mid n$. However, if $\ell_{1}=2$ or $\ell_{2}=2$, then it is possible that $36 \nmid n$.

Suppose $\ell_{1}=\ell_{2}=2$ and $n=6 s$ where $s$ is a product of primes powers not involving 2 or 3 , then $36 \nmid n$. There exists an $\mathrm{OA}(3,6)$ and 3-MOLS $(s)$. Apply Lemma 6.18 with $m=$ 6 for all $s>36$ and $s \equiv 1$ or $35(\bmod 36)$. If $(s, n) \in\{(7,42),(11,66),(13,78),(17,102)$, $(19,114),(23,138),(25,150),(29,174),(31,186),(35,210)\}$, then we get the following open cases: $(a, b ; n) \in\{(36,49 ; 42),(36,121 ; 66),(36,169 ; 78),(36,289 ; 102)$,

$(36,361 ; 114),(36,529 ; 138),(36,625 ; 150),(36,841 ; 174),(36,961 ; 186),(36,1225 ; 210)\}$. If $(s, n)=(5,30)$, then $a>b$ and we need not consider this case. If $s>36$ and $s \equiv 5,7,11,13,17,19,23,25,29,31(\bmod 36)$, then we have open cases.

Suppose $\ell_{1}>2$ but $\ell_{2}=2$. Then $a=2^{\ell_{1}} \cdot 9$. If $n=3 s$ where $s$ is a product of prime powers not involving 3 but involving 4 , then $36 \nmid n$. However, in this case we may write $n=12 s_{1}$ where $s_{1}$ is a product of prime powers not involving 3 . Hence, if $s_{1} \neq 2$, then we may let $h=4$ and $k=3 s_{1}$ and apply Lemma 6.22 to obtain $N_{\text {MOELR }}(a, b ; n) \geq 3$. If $s_{1}=2$, then $n=a=72$, and because $a \mid n$, we may apply Lemma 6.11 
Suppose $\ell_{2}>2$ but $\ell_{1}=2$. Then $a=4 \cdot 3^{\ell_{2}}$. If $n=2 s$ where $s$ is an odd product of prime powers involving 9 , then $36 \nmid n$. However, in this case we may write $n=18 s_{1}$ where $s_{1}$ is an odd product of prime powers. Then, if $s_{1} \neq 3,5$, we set $h=9$ and $k=2 s_{1}$ and apply Lemma 6.22 to obtain $N_{\operatorname{MOELR}}(a, b ; n) \geq 3$. If $s_{1}=3$ or 5 , then $n=54$ or 90 respectively and $a>b$, so we need not consider these cases.

Suppose $n=p_{0}^{a_{1}} p_{1}^{a_{1}} \cdots p_{t}^{a_{t}}$ where $p_{0}<p_{1}<\cdots<p_{t}$ are all prime. Without loss of generality, we can assume $a \leq b$, for if $a>b$, then we could apply Lemma 6.9. So because we assume $a \leq b$ and $n^{2}=a b$, we need only consider $a$ such that $a \leq n$. If $n=p_{i}^{a_{i}}$, then apply Corollary 6.13. If $p_{i} \neq 2$ or 3 for any $i$, then apply Lemma6.24. If $p_{0}=2$ and $p_{i} \neq 3$ for any $i$, then apply Lemma 6.25. If $p_{0}=3$ and $p_{i} \neq 2$, then apply Lemma 6.26. Finally if $p_{0}=2$ and $p_{1}=3$, then apply Lemma 6.27.

By combining the results from the above lemmas, if $n \geq 216$ we can construct a $3-\operatorname{MOELR}(a, b ; n)$ for all $a$ and $b$ such that $n^{2}=a b$ with some exceptions. Notice that we can often get more than 3 , but we are always guaranteed to get 3 . Thus we have the main theorem.

Theorem 6.28. If $n^{2}=a b$ and $n \geq 216$, then $N_{\text {MOELR }}(a, b ; n) \geq 3$ except possibly when

$$
\begin{aligned}
& a=9 \text { and } n=3 s, \text { where } s \equiv 2,4,5,7(\bmod 9), s>9 ; \\
& a=18 \text { and } n=3 s, \text { where } s \equiv 4,8,10,14(\bmod 18), s>18 ; \\
& a=36 \text { and } n=6 s, \text { where } s \equiv 5,7,11,13,17,19,23,25,29,31(\bmod 36), s>36 ;
\end{aligned}
$$

Furthermore, if $n<216$, then $N_{\operatorname{MOELR}}(a, b ; n) \geq 3$ except possibly when $(a, b ; n)$ is one of the following.
$(2,18 ; 6)$
$(18,32 ; 24)$
$(36,121 ; 66)$
$(36,961 ; 186)$
$(2,50 ; 10)$
$(18,50 ; 30)$
$(36,169 ; 78)$
$(36,1225 ; 210)$
$(8,18 ; 12)$
$(18,98 ; 42)$
$(36,289 ; 102)$
$(49,100 ; 70)$
$(9,16 ; 12)$
$(18,128 ; 48)$
$(36,361 ; 114)$
$(81,100 ; 90)$
$(9,25 ; 15)$
$(25,36 ; 30)$
$(36,529 ; 138)$
$(9,49 ; 21)$
$(27,300 ; 90)$
$(36,625 ; 150)$
$(12,27 ; 18)$
$(36,49 ; 42)$
$(36,841 ; 174)$

Also, $N_{\text {MOelR }}(2,2 ; 2)=1, N_{\text {MOelR }}(3,3 ; 3)=2, N_{\text {MOelR }}(6,6 ; 6)=1$, and $N_{\text {MOELR }}(10,10 ; 10)=2$. 
122 


\section{Appendix A}

\section{Table of $\operatorname{MOELR}(a, b ; n)$ where $n \leq 216$}

The following is a list of the MOELRs that have been solved. Make note that $\checkmark$ implies that every dimension of $A$ with the given $n$ symbols has a solution. The notation $3-(a, b ; n)$ represents a $3-\operatorname{MOELR}(a, b ; n)$ and was used to conserve space. The list of $n \leq 216$ moves incrementally from 2 to 144 and then skips to $n$ values where $a=36$. The column headings named $N$ indicate $N_{\text {MOELR }}$. 


\begin{tabular}{|c|c|c|c|}
\hline$n$ & $a \times b$ & $N$ & Construction \\
\hline 2 & $2 \times 2$ & $\checkmark$ & Corollary 6.13 \\
\hline 3 & $3 \times 3$ & $\checkmark$ & Corollary $\overline{6.13}$ \\
\hline 4 & All possible & $\checkmark$ & Corollary 6.13 \\
\hline 5 & $5 \times 5$ & $\checkmark$ & Corollary 6.13 \\
\hline \multirow[t]{4}{*}{6} & $2 \times 18$ & & \\
\hline & $3 \times 12$ & 3 & $\mathrm{C}$ \\
\hline & $4 \times 9$ & 3 & $\mathrm{C}$ \\
\hline & $6 \times 6$ & 1 & [21] \\
\hline 7 & $7 \times 7$ & $\checkmark$ & Corollary 6.13 \\
\hline 8 & All possible & $\checkmark$ & Corollary $\overline{6.13}$ \\
\hline 9 & All possible & $\checkmark$ & Corollary 6.13 \\
\hline \multirow[t]{4}{*}{10} & $2 \times 50$ & & \\
\hline & $4 \times 25$ & 3 & Example 6.7 \\
\hline & $5 \times 20$ & 3 & Lemma 6.14 \\
\hline & $10 \times 10$ & 2 & [10] \\
\hline 11 & $11 \times 11$ & $\checkmark$ & Corollary 6.13 \\
\hline \multirow[t]{7}{*}{12} & $2 \times 72$ & 5 & Lemma 6.11 \\
\hline & $3 \times 48$ & 5 & Lemma 6.11 \\
\hline & $4 \times 36$ & 5 & Lemma 6.11 \\
\hline & $6 \times 24$ & 5 & Lemma $\overline{6.11}$ \\
\hline & $8 \times 18$ & & \\
\hline & $9 \times 16$ & 3 & Lemma 6.14 \\
\hline & $12 \times 12$ & 5 & [30] \\
\hline 13 & $13 \times 13$ & $\checkmark$ & Corollary 6.13 \\
\hline \multirow[t]{4}{*}{14} & $2 \times 98$ & 3 & Lemma 6.11 \\
\hline & $4 \times 49$ & & \\
\hline & $7 \times 28$ & 3 & Lemma 6.11 \\
\hline & $14 \times 14$ & 3 & [46] \\
\hline \multirow[t]{4}{*}{15} & $3 \times 75$ & 4 & Lemma 6.11 \\
\hline & $5 \times 45$ & 4 & Lemma \\
\hline & $9 \times 25$ & & \\
\hline & $15 \times 15$ & 4 & {$[1,44]$} \\
\hline 16 & All possible & $\checkmark$ & Corollary 6.13 \\
\hline 17 & $17 \times 17$ & $\checkmark$ & Corollary 6.13 \\
\hline \multirow[t]{7}{*}{18} & $2 \times 162$ & 3 & Theorem 6.11 \\
\hline & $3 \times 108$ & 3 & Lemma 6.11 \\
\hline & $4 \times 81$ & 3 & Lemma 6.16 \\
\hline & $6 \times 54$ & 3 & Lemma \\
\hline & $9 \times 36$ & 3 & Lemma \\
\hline & $12 \times 27$ & & \\
\hline & $18 \times 18$ & 3 & [49] \\
\hline 19 & $19 \times 19$ & $\checkmark$ & Corollary 6.13 \\
\hline
\end{tabular}

\begin{tabular}{|c|c|c|c|}
\hline$n$ & $a \times b$ & $N$ & Construction \\
\hline \multirow[t]{7}{*}{20} & $2 \times 200$ & 4 & Lemma 6.11 \\
\hline & $4 \times 100$ & 4 & Lemma 6.11 \\
\hline & $5 \times 80$ & 4 & Lemma $\overline{6.11}$ \\
\hline & $8 \times 50$ & 3 & Lemma $\overline{6.22}$ \\
\hline & $10 \times 40$ & 4 & Lemma $\longdiv { 6 . 1 1 }$ \\
\hline & $16 \times 25$ & 3 & Lemma $\overline{6.22}$ \\
\hline & $20 \times 20$ & 4 & [8, 47] \\
\hline \multirow[t]{4}{*}{21} & $3 \times 147$ & 5 & $\begin{array}{ll}\text { Lemma } 6.11 \\
\end{array}$ \\
\hline & $7 \times 63$ & 5 & Lemma $\overline{6.11}$ \\
\hline & $9 \times 49$ & & \\
\hline & $21 \times 21$ & 5 & [40] \\
\hline \multirow[t]{4}{*}{22} & $2 \times 242$ & 3 & Lemma 6.11 \\
\hline & $4 \times 121$ & 3 & Lemma 6.16 \\
\hline & $11 \times 44$ & 3 & Lemma 6.11 \\
\hline & $22 \times 22$ & 3 & {$[2,9]$} \\
\hline 23 & $23 \times 23$ & $\checkmark$ & Corollary 6.13 \\
\hline \multirow[t]{12}{*}{24} & $2 \times 288$ & 6 & Lemma 6.11 \\
\hline & $3 \times 192$ & 6 & Lemma 6.11 \\
\hline & $4 \times 144$ & 6 & Lemma $\overline{6.11}$ \\
\hline & $6 \times 96$ & 6 & Lemma 6.11 \\
\hline & $8 \times 72$ & 6 & Lemma $\overline{6.11}$ \\
\hline & & & Lemma 6.14 \\
\hline & $9 \times 64$ & 3 & $k=3, a=9$ \\
\hline & & & $b=16, n=12$ \\
\hline & $12 \times 48$ & 6 & Lemma 6.11 \\
\hline & $16 \times 36$ & 3 & Lemma 6.20 \\
\hline & $18 \times 32$ & & \\
\hline & $24 \times 24$ & 6 & [6] \\
\hline 25 & All possible & $\checkmark$ & Corollary 6.13 \\
\hline \multirow[t]{4}{*}{26} & $2 \times 338$ & 4 & Lemma 6.11 \\
\hline & $4 \times 169$ & 3 & Lemma 6.16 \\
\hline & $13 \times 52$ & 4 & Lemma 6.11 \\
\hline & $26 \times 26$ & 4 & [17] \\
\hline 27 & All possible & $\checkmark$ & Corollary 6.13 \\
\hline
\end{tabular}




\begin{tabular}{|c|c|c|c|}
\hline$n$ & $a \times b$ & $N$ & Construction \\
\hline \multirow[t]{8}{*}{28} & $2 \times 392$ & $\overline{5}$ & Lemma 6.11 \\
\hline & $4 \times 196$ & 5 & Lemma 6.11 \\
\hline & $7 \times 112$ & 5 & Lemma \\
\hline & $8 \times 98$ & 3 & Lemma 6.22 \\
\hline & $14 \times 56$ & 5 & Lemma 6.11 \\
\hline & & & Jemmo \\
\hline & $16 \times 49$ & 3 & $\begin{array}{l}\text { Lemma } \\
h=4, k=7\end{array}$ \\
\hline & $28 \times 28$ & 5 & [1] \\
\hline 29 & $29 \times 29$ & $\checkmark$ & Corollary 6.13 \\
\hline \multirow[t]{20}{*}{30} & $2 \times 450$ & 4 & Lemma 6.11 \\
\hline & $3 \times 300$ & 4 & Lemma \\
\hline & $4 \times 225$ & 3 & Lemma 6.16 , \\
\hline & & & $m=2, s=15$ \\
\hline & $5 \times 180$ & 4 & Lemma 6.11 \\
\hline & $6 \times 150$ & 4 & Lemma \\
\hline & & & If $3-(9,25 ; 15)$ \\
\hline & $9 \times 100$ & & exists, then \\
\hline & & & Lemma 6.14 \\
\hline & $10 \times 90$ & 4 & Lemma 6.11 \\
\hline & $12 \times 75$ & 3 & Lemma 6.16 \\
\hline & & & $m=2, s=15$ \\
\hline & $15 \times 60$ & 4 & Lemma 6.11 \\
\hline & $18 \times 50$ & & \\
\hline & $20 \times 45$ & 3 & Lemma 6.20 \\
\hline & $20 \times 45$ & 5 & $t=5$ \\
\hline & & & If $\exists 3-(25,9 ; 15)$ \\
\hline & $25 \times 36$ & & then use \\
\hline & & & Lemma 6.14 . \\
\hline & $30 \times 30$ & 4 & [8] \\
\hline 31 & $31 \times 31$ & $\checkmark$ & Corollary 6.13 \\
\hline 32 & All possible & $\checkmark$ & Corollary 6.13 \\
\hline \multirow[t]{4}{*}{33} & $3 \times 363$ & 5 & Lemma 6.11 \\
\hline & $9 \times 121$ & & \\
\hline & $11 \times 99$ & 5 & Lemma 6.11 \\
\hline & $33 \times 33$ & 5 & [7] \\
\hline \multirow[t]{4}{*}{34} & $2 \times 578$ & 4 & $\begin{array}{ll} & \\
\end{array}$ \\
\hline & $4 \times 289$ & 3 & $\begin{array}{l}\text { Lemma } 6.16 \\
m=2, s=17\end{array}$ \\
\hline & $17 \times 68$ & 4 & Lemma 6.11 \\
\hline & $34 \times 34$ & 4 & [1] \\
\hline
\end{tabular}

\begin{tabular}{|c|c|c|c|}
\hline$n$ & $a \times b$ & $N$ & Construction \\
\hline \multirow[t]{5}{*}{35} & $5 \times 245$ & 5 & Lemma 6.11 \\
\hline & $7 \times 175$ & 5 & Lemma 6.11 \\
\hline & $25 \times 10$ & 4 & Lemma 6.22, \\
\hline & $20 \times 49$ & 4 & $h=5, k=7$ \\
\hline & $34 \times 34$ & 5 & [53] \\
\hline \multirow[t]{14}{*}{36} & $2 \times 648$ & 8 & Lemma 6.11 \\
\hline & $3 \times 432$ & 8 & Lemma 6.11 \\
\hline & $4 \times 324$ & 8 & Lemma $\overline{\overline{6.11}}$ \\
\hline & $8 \times 162$ & 3 & Lemma 6.22; \\
\hline & $9 \times 144$ & 8 & Lemma 611 \\
\hline & $12 \times 108$ & 8 & Lemma $\overline{\overline{611}}$ \\
\hline & $12 \times 100$ & 0 & Lemma \\
\hline & $16 \times 81$ & 3 & $h=4, k=9$ \\
\hline & $18 \times 72$ & 8 & Lemma 6.11 \\
\hline & $24 \times 54$ & 3 & Lemma 6.22 \\
\hline & $24 \times 04$ & & $h=4, k=9$ \\
\hline & $27 \times 48$ & 3 & Lemma 6.22: \\
\hline & $26 \times 26$ & & $h=9, k=4$ \\
\hline & $30 \times 30$ & 8 & {$[0]$} \\
\hline 37 & $37 \times 37$ & $\checkmark$ & Corollary 6.13 \\
\hline \multirow[t]{5}{*}{38} & $2 \times 722$ & 4 & Lemma 6.11 \\
\hline & $4 \times 361$ & 3 & Lemma 6.16 \\
\hline & & & $m=2, s=19$ \\
\hline & $19 \times 76$ & 4 & Lemma 6.11 \\
\hline & $38 \times 38$ & 4 & [8] \\
\hline \multirow[t]{4}{*}{39} & $3 \times 507$ & 5 & Lemma 6.11 \\
\hline & $9 \times 169$ & & \\
\hline & $13 \times 117$ & 5 & Lemma 6.11 \\
\hline & $39 \times 39$ & 5 & [7] \\
\hline \multirow[t]{12}{*}{40} & $2 \times 800$ & 7 & Lemma 6.11 \\
\hline & $4 \times 400$ & 7 & Lemma 6.11 \\
\hline & $5 \times 320$ & 7 & Lemma 6.11 \\
\hline & $8 \times 200$ & 7 & Lemma 6.11 \\
\hline & $10 \times 160$ & 7 & Lemma 6.11 \\
\hline & $16 \times 100$ & 3 & Lemma 6.22, \\
\hline & $20 \times 80$ & 7 & Lemma 6.11 \\
\hline & & & Lemma 6.22 \\
\hline & $25 \times 64$ & 4 & $h=5, k=8$ \\
\hline & $32 \times 50$ & 4 & Lemma 6.22 \\
\hline & & & $h=8, k=5$ \\
\hline & $28 \times 28$ & 7 & [5] \\
\hline 41 & $41 \times 41$ & $\checkmark$ & Corollary 6.13 \\
\hline
\end{tabular}




\begin{tabular}{|c|c|c|c|}
\hline$n$ & $a \times b$ & $N$ & Construction \\
\hline \multirow[t]{15}{*}{42} & $2 \times 882$ & 5 & Lemma 6.11 \\
\hline & $3 \times 588$ & 5 & Lemma 6.11 \\
\hline & $4 \times 441$ & 3 & Lemma 6.16; \\
\hline & $4 \times 441$ & & $m=2, s=21$ \\
\hline & $7 \times 252$ & 5 & Lemma 6.11 \\
\hline & $9 \times 196$ & & If $\exists 3-(9,49 ; 21)$ \\
\hline & & & Lemma \\
\hline & $12 \times 147$ & 3 & $m=2, s=21$ \\
\hline & $14 \times 126$ & 5 & Lemma $6.11 \mid$ \\
\hline & $18 \times 98$ & & \\
\hline & $21 \times 84$ & 5 & Lemma 6.11 \\
\hline & $28 \times 63$ & 3 & Lemma 6.20 \\
\hline & & & $t=7,3-(4,9 ; 6)$ \\
\hline & $36 \times 49$ & & \\
\hline & $42 \times 42$ & 5 & [1] \\
\hline 43 & $43 \times 43$ & $\checkmark$ & Corollary 6.13 \\
\hline \multirow[t]{8}{*}{44} & $2 \times 968$ & 5 & Lemma 6.11 \\
\hline & $4 \times 484$ & 5 & Lemma 6.11 \\
\hline & $8 \times 242$ & 3 & Lemma 6.16; \\
\hline & $11 \times 176$ & & $m=2, s=22$ \\
\hline & $11 \times 176$ & 5 & Lemma 6.11 \\
\hline & $16 \times 121$ & 3 & $\begin{array}{l}\text { Lemma } 6.22\} \\
h=4 k=11\end{array}$ \\
\hline & $22 \times 88$ & 5 & Lemma 6.11 \\
\hline & $44 \times 44$ & 5 & [1] \\
\hline \multirow[t]{9}{*}{45} & $3 \times 675$ & 6 & Lemma 6.11 \\
\hline & $5 \times 289$ & 6 & Lemma $\overline{6.11}$ \\
\hline & $9 \times 68$ & 6 & Lemma $\overline{6.11}$ \\
\hline & $15 \times 135$ & 6 & Lemma 6.11 \\
\hline & $25 \times 81$ & 4 & Lemma 6.22 \\
\hline & & & $h=5, k=9$ \\
\hline & $27 \times 75$ & 4 & Lemma 6.22; \\
\hline & 15 & 6 & $n=y, k=5$ \\
\hline & 40 人 & 0 & \\
\hline \multirow[t]{4}{*}{46} & $2 \times 1058$ & 4 & Lemma 6.11 \\
\hline & $4 \times 529$ & 3 & $\begin{array}{l}\text { Lemma 6.16; } \\
m=2 s=23\end{array}$ \\
\hline & $23 \times 92$ & 4 & Lemma 6.11 \\
\hline & $46 \times 46$ & 4 & [18] \\
\hline 47 & $47 \times 47$ & $\checkmark$ & $\begin{array}{l}\text { Corollary } 6.13 \\
\end{array}$ \\
\hline
\end{tabular}

\begin{tabular}{|c|c|c|c|}
\hline$n$ & $a \times b$ & $N$ & Construction \\
\hline \multirow[t]{11}{*}{$\overline{48}$} & $2 \times 1152$ & $\overline{8}$ & Lemma 6.11 \\
\hline & $3 \times 768$ & 8 & Lemma 6.11 \\
\hline & $4 \times 576$ & 8 & Lemma $\overline{6.11}$ \\
\hline & $8 \times 288$ & 8 & Lemma 6.11 \\
\hline & $9 \times 256$ & & \\
\hline & $16 \times 144$ & 3 & $\begin{array}{l}\text { Lemma 6.22: } \\
h=4 k=12\end{array}$ \\
\hline & $18 \times 128$ & & \\
\hline & $24 \times 96$ & 8 & Lemma 6.11 \\
\hline & $32 \times 72$ & 3 & $\begin{array}{l}\text { Lemma 6.22 } \\
h=4, k=12\end{array}$ \\
\hline & $36 \times 64$ & 3 & $h=4, k=12$ \\
\hline & $48 \times 48$ & 8 & [4] \\
\hline 49 & All possible & $\checkmark$ & Corollary 6.13 \\
\hline \multirow[t]{7}{*}{50} & $2 \times 800$ & 6 & Lemma 6.11 \\
\hline & $4 \times 625$ & 3 & Lemma 6.16 \\
\hline & $5 \times 500$ & 6 & Lemma 6.11 \\
\hline & $10 \times 250$ & 6 & Lemma $\overline{6.11}$ \\
\hline & $20 \times 125$ & 3 & Lemma 6.20 \\
\hline & $25 \times 100$ & 6 & $\begin{array}{c}t=5,3-(4,25 ; 10) \\
\text { Lemma } 6.11\end{array}$ \\
\hline & $50 \times 50$ & 6 & [18] \\
\hline \multirow[t]{5}{*}{51} & $3 \times 867$ & 5 & Lemma 6.11 \\
\hline & $9 \times 289$ & 3 & Lemma 6.17 \\
\hline & & & $m=3, s=17$ \\
\hline & $17 \times 153$ & 5 & Lemma 6.11 \\
\hline & $51 \times 51$ & 5 & [7] \\
\hline \multirow[t]{7}{*}{52} & $2 \times 1352$ & 5 & Lemma 6.11 \\
\hline & $4 \times 676$ & 5 & Lemma 6.11 \\
\hline & $8 \times 338$ & 3 & $\begin{array}{l}\text { Lemma } 6.22 \\
h=4, k=13\end{array}$ \\
\hline & $13 \times 208$ & 5 & Lemma 6.11 \\
\hline & $16 \times 169$ & 3 & $\begin{array}{l}\text { Lemma 6.22 } \\
h=4 k=13\end{array}$ \\
\hline & $26 \times 104$ & 5 & Lemma 6.11 \\
\hline & $52 \times 52$ & 5 & [1] \\
\hline 53 & $53 \times 53$ & $\checkmark$ & \begin{tabular}{|l|l|} 
Corollary & 6.13 \\
\end{tabular} \\
\hline
\end{tabular}




\begin{tabular}{|c|c|c|c|}
\hline$n$ & $a \times b$ & $N$ & Construction \\
\hline \multirow[t]{13}{*}{54} & $2 \times 1458$ & 5 & Lemma 6.11 \\
\hline & $3 \times 972$ & 5 & Lemma 6.11 \\
\hline & $4 \times 729$ & 3 & Lemma 6.16; \\
\hline & & & $m=2, s=27$ \\
\hline & $6 \times 486$ & 5 & Lemma 6.11 \\
\hline & $9 \times 324$ & 3 & Lemma 6.11 \\
\hline & $12 \times 243$ & 3 & Lemma 6.16 \\
\hline & & & $m=2, s=27$ \\
\hline & $18 \times 162$ & 3 & Lemma 6.11: \\
\hline & $27 \times 108$ & 5 & Lemma 6.11 \\
\hline & $36 \times 81$ & 3 & Lemma 6.20 \\
\hline & 的 & & $t=9,3-(4,9 ; 6)$ \\
\hline & $54 \times 54$ & 5 & [1] \\
\hline \multirow[t]{5}{*}{55} & $5 \times 605$ & 6 & Lemma 6.11 \\
\hline & $11 \times 275$ & 6 & Lemma 6.11 \\
\hline & $25 \times 121$ & 4 & Lemma 6.22, \\
\hline & & & $h=5, k=11$ \\
\hline & $55 \times 55$ & 6 & [54] \\
\hline \multirow[t]{13}{*}{56} & $2 \times 1568$ & 7 & Lemma 6.11 \\
\hline & $4 \times 784$ & 7 & Lemma $\overline{6.11}$ \\
\hline & $7 \times 448$ & 7 & Lemma 6.11 \\
\hline & $8 \times 392$ & 7 & Lemma 6.11 \\
\hline & $14 \times 224$ & 7 & Lemma 6.11 \\
\hline & $16 \times 106$ & 6 & Lemma 6.22 \\
\hline & $10 \times 190$ & 0 & $h=8, k=7$ \\
\hline & $28 \times 112$ & 7 & Lemma 6.11 \\
\hline & $32 \times 08$ & 6 & Lemma 6.22, \\
\hline & $52 \times 50$ & 0 & $h=8, k=7$ \\
\hline & $49 \times 64$ & 6 & Lemma 6.22; \\
\hline & & & $h=7, k=8$ \\
\hline & $56 \times 56$ & 7 & [2, 37] \\
\hline \multirow[t]{4}{*}{57} & $3 \times 1083$ & 7 & Lemma 6.11 \\
\hline & $9 \times 361$ & 4 & Lemma 6.16; \\
\hline & $19 \times 171$ & 7 & Lemma 6.11 \\
\hline & $57 \times 57$ & 7 & [18] \\
\hline \multirow[t]{4}{*}{58} & $2 \times 1682$ & 5 & Lemma 6.11 \\
\hline & $4 \times 841$ & 3 & $\begin{array}{l}\text { Lemma } 6.16 \\
m=2, s=29\end{array}$ \\
\hline & $29 \times 116$ & 5 & Lemma 6.11 \\
\hline & $58 \times 58$ & 5 & [18] \\
\hline 59 & $59 \times 59$ & $\checkmark$ & Corollary 6.13 \\
\hline
\end{tabular}

\begin{tabular}{|c|c|c|c|}
\hline$n$ & $a \times b$ & $N$ & Construction \\
\hline \multirow[t]{22}{*}{60} & $2 \times 1800$ & 4 & Lemma 6.11 \\
\hline & $3 \times 1200$ & 4 & Lemma 6.11 \\
\hline & $4 \times 900$ & 4 & Lemma $\overline{6.11}$ \\
\hline & $5 \times 720$ & 4 & Lemma 6.11 \\
\hline & $6 \times 600$ & 4 & Lemma 6.11 \\
\hline & $8 \times 450$ & 3 & Lemma 6.22 \\
\hline & & & $\begin{array}{l}h=4, k=15 \\
\text { Lemma } 6.16\end{array}$ \\
\hline & $9 \times 400$ & 4 & $m=3, s=20$ \\
\hline & $10 \times 360$ & 4 & Lemma 6.11 \\
\hline & $12 \times 300$ & 4 & Lemma $\overline{6.11}$ \\
\hline & $15 \times 240$ & 4 & Lemma 6.11 \\
\hline & $16 \times 225$ & 3 & $\begin{array}{l}\text { Lemma 6.22 } \\
h=4, k=15\end{array}$ \\
\hline & $18 \times 200$ & 3 & $\begin{array}{l}\text { Lemma 6.17 } \\
m=3, s=20\end{array}$ \\
\hline & $20 \times 180$ & 4 & Lemma 6.11 \\
\hline & $24 \times 150$ & 4 & $\begin{array}{l}\text { Lemma 6.22 } \\
h=12, k=5\end{array}$ \\
\hline & $25 \times 144$ & 4 & $\begin{array}{l}\text { Lemma 6.22 } \\
h=5, k=12\end{array}$ \\
\hline & $30 \times 120$ & 4 & Lemma 6.11 \\
\hline & $36 \times 100$ & 4 & $\begin{array}{l}\text { Lemma 6.22 } \\
h=12, k=5\end{array}$ \\
\hline & $45 \times 80$ & 4 & $\begin{array}{l}\text { Lemma 6.22 } \\
h=5, k=12\end{array}$ \\
\hline & $48 \times 75$ & 4 & $\begin{array}{l}\text { Lemma } 6.22 \\
h=12, k=5\end{array}$ \\
\hline & $50 \times 72$ & 4 & $\begin{array}{l}\text { Lemma 6.22 } \\
h=5, k=12\end{array}$ \\
\hline & $60 \times 60$ & 4 & [18] \\
\hline 61 & $61 \times 61$ & $\checkmark$ & Corollary 6.13 \\
\hline \multirow[t]{4}{*}{62} & $2 \times 1922$ & 5 & Lemma 6.11 \\
\hline & $4 \times 961$ & 3 & $\begin{array}{l}\text { Lemma 6.16 } \\
m=2, s=31\end{array}$ \\
\hline & $31 \times 124$ & 5 & Lemma 6.11 \\
\hline & $62 \times 62$ & 5 & [1] \\
\hline \multirow[t]{6}{*}{63} & $3 \times 1323$ & 6 & Lemma 6.11 \\
\hline & $7 \times 567$ & 6 & Lemma $\overline{6.11}$ \\
\hline & $9 \times 441$ & 6 & Lemma 6.11 \\
\hline & $21 \times 189$ & 6 & Lemma 6.11 \\
\hline & $27 \times 147$ & 6 & $\begin{array}{l}\text { Lemma 6.22 } \\
h=9, k=7\end{array}$ \\
\hline & $63 \times 63$ & 6 & [18] \\
\hline 64 & All possible & $\checkmark$ & Corollary 6.13 \\
\hline
\end{tabular}




\begin{tabular}{|c|c|c|c|}
\hline$n$ & $a \times b$ & $N$ & Construction \\
\hline \multirow[t]{5}{*}{65} & $\overline{5 \times 845}$ & $\overline{c 6}$ & Lemma 6.11 \\
\hline & $13 \times 325$ & 6 & Lemma 6.11 \\
\hline & $25 \times 169$ & 4 & Lemma 6.22 \\
\hline & & & $h=5, k=13$ \\
\hline & $65 \times 65$ & 6 & [18] \\
\hline \multirow[t]{14}{*}{66} & $2 \times 2178$ & 5 & Lemma 6.11 \\
\hline & $3 \times 1452$ & 5 & Lemma \\
\hline & $4 \times 1089$ & 3 & Lemma 6.16 \\
\hline & $4 \times 1009$ & 5 & $m=2, s=33$ \\
\hline & $6 \times 726$ & 5 & Lemma 6.11 \\
\hline & $9 \times 484$ & & \\
\hline & $11 \times 396$ & 5 & Lemma 6.11 \\
\hline & $12 \times 363$ & 3 & Lemma 6.16; \\
\hline & $18 \times 242$ & & $m=2, s=33$ \\
\hline & $22 \times 198$ & 5 & Lemma 6.11 \\
\hline & $33 \times 132$ & 5 & Lemma 6.11 \\
\hline & $36 \times 121$ & & \\
\hline & $44 \times 99$ & 3 & Lemma 6.20 \\
\hline & $66 \times 66$ & 5 & $\begin{array}{c}t=11,3-(4,9 ; 6) \\
{[18]}\end{array}$ \\
\hline 67 & $67 \times 67$ & $\checkmark$ & \begin{tabular}{l|l} 
Corollary 6.13 \\
\end{tabular} \\
\hline \multirow[t]{8}{*}{68} & $2 \times 2312$ & 5 & Lemma 6.11 \\
\hline & $4 \times 1156$ & 5 & Lemma 6.11 \\
\hline & $8 \times 578$ & 3 & Lemma 6.22; \\
\hline & 0 人 & & $h=4, k=17$ \\
\hline & $16 \times 289$ & 3 & $\begin{array}{l}\text { Lemma } 6.22 \\
h=4, k=17\end{array}$ \\
\hline & $17 \times 272$ & 5 & Lemma 6.11 \\
\hline & $34 \times 136$ & 5 & Lemma \\
\hline & $68 \times 68$ & 5 & [18] \\
\hline \multirow[t]{3}{*}{69} & $\begin{array}{c}3 \times 1587 \\
9 \times 599\end{array}$ & 6 & Lemma 6.11 \\
\hline & $23 \times 207$ & 6 & Lemma 6.11 \\
\hline & $69 \times 69$ & 6 & {$[18]$} \\
\hline
\end{tabular}

\begin{tabular}{|c|c|c|c|}
\hline$n$ & $a \times b$ & $N$ & Construction \\
\hline \multirow[t]{17}{*}{70} & $2 \times 2450$ & 6 & Lemma 6.11 \\
\hline & $4 \times 1225$ & 3 & Lemma 6.16 \\
\hline & $4 \times 1225$ & 3 & $m=2, s=35$ \\
\hline & $5 \times 980$ & 6 & Lemma 6.11 \\
\hline & $7 \times 700$ & 6 & Lemma 6.11 \\
\hline & $10 \times 490$ & 6 & Lemma 6.11 \\
\hline & $14 \times 350$ & 6 & Lemma 6.11 \\
\hline & $20 \times 245$ & 3 & Lemma 6.22; \\
\hline & & & $h=5, k=14$ \\
\hline & $25 \times 196$ & 3 & Lemma 6.22; \\
\hline & & & $h=5, k=14$ \\
\hline & $28 \times 175$ & 3 & Lemma 6.22 ; \\
\hline & $25 \vee 140$ & 6 & - \\
\hline & $49 \times 100$ & & \\
\hline & & & I emma 620 \\
\hline & $50 \times 98$ & 3 & $h=5, k=14$ \\
\hline & $70 \times 70$ & 6 & [18] \\
\hline 71 & $71 \times 71$ & $\checkmark$ & Corollary 6.13 \\
\hline \multirow[t]{21}{*}{72} & $2 \times 2592$ & 7 & Lemma 6.11 \\
\hline & $3 \times 1728$ & 7 & Lemma 6.11 \\
\hline & $4 \times 1296$ & 7 & Lemma 6.11 \\
\hline & $6 \times 864$ & 7 & Lemma \\
\hline & $8 \times 648$ & 7 & Lemma 6.11 \\
\hline & $9 \times 576$ & 7 & Lemma 6.11 \\
\hline & $12 \times 432$ & 7 & Lemma 6.11 \\
\hline & $16 \times 324$ & 7 & Lemma 6.22 \\
\hline & 280 & 7 & $n=8, \kappa=y$ \\
\hline & $24 \times 216$ & 7 & Lemma \\
\hline & & & Jemma \\
\hline & $27 \times 192$ & 7 & $h=9, k=8$ \\
\hline & & 7 & Lemma 6.22; \\
\hline & & & $h=8, k=9$ \\
\hline & $36 \times 144$ & 7 & Lemma 6.11 \\
\hline & $48 \times 108$ & 7 & Lemma 6.22, \\
\hline & & & $h=8, k=9$ \\
\hline & $54 \times 96$ & 7 & $\begin{array}{l}\text { Lemma 6.22; } \\
h=9, k=8\end{array}$ \\
\hline & 61 & 7 & Lemma 6.22, \\
\hline & & 1 & $h=8, k=9$ \\
\hline & $72 \times 72$ & 7 & {$[18]$} \\
\hline 73 & $73 \times 73$ & $\checkmark$ & Corollary $\longdiv { 6 . 1 3 }$ \\
\hline
\end{tabular}




\begin{tabular}{|c|c|c|c|}
\hline$n$ & $a \times b$ & $N$ & Construction \\
\hline \multirow[t]{5}{*}{74} & $2+2 \times 2738$ & $\overline{5}$ & $\begin{array}{lll}\text { Lemma } 6.11 \\
\end{array}$ \\
\hline & $4 \vee 1260$ & 3 & Lemma 6.16 \\
\hline & $4 \times 1309$ & 3 & $m=2, s=37$ \\
\hline & $37 \times 148$ & 5 & Lemma 6.11 \\
\hline & $74 \times 74$ & 5 & [18] \\
\hline \multirow[t]{8}{*}{75} & $3 \times 1875$ & 7 & Lemma 6.11 \\
\hline & $5 \times 1125$ & 7 & Lemma 6.11 \\
\hline & $9 \times 625$ & & \\
\hline & $15 \times 375$ & 7 & Lemma 6.11 \\
\hline & $25 \times 225$ & 4 & Lemma 6.11 \\
\hline & $45 \times 125$ & 4 & Lemma 6.22, \\
\hline & $40 \times 120$ & & $h=15, k=5$ \\
\hline & $75 \times 75$ & 7 & [6] \\
\hline \multirow[t]{8}{*}{76} & $2 \times 2888$ & 6 & Lemma 6.11 \\
\hline & $4 \times 1444$ & 6 & Lemma 6.11 \\
\hline & $8 \times 722$ & 3 & Lemma 6.22 , \\
\hline & $0 \times 122$ & & $h=4, k=19$ \\
\hline & $16 \times 361$ & 3 & $\begin{array}{l}\text { Lemma } 6.22 \\
h=4 k=19\end{array}$ \\
\hline & $19 \times 304$ & 6 & Lemma 6.11 \\
\hline & $38 \times 152$ & 6 & Lemma \\
\hline & $76 \times 76$ & 6 & [18] \\
\hline \multirow[t]{4}{*}{77} & $7 \times 847$ & 6 & Lemma 6.11 \\
\hline & $11 \times 539$ & 6 & Lemma 6.11 \\
\hline & $49 \times 121$ & 6 & Lemma 6.22 \\
\hline & $77 \times 77$ & 6 & $h=7, k=11$ \\
\hline \multirow[t]{15}{*}{78} & $2 \times 3042$ & 6 & Lemma 6.11 \\
\hline & $3 \times 2028$ & 6 & Lemma 6.11 \\
\hline & $4 \times 1521$ & 3 & Lemma \\
\hline & $4 \times 1521$ & 3 & $m=2, s=39$ \\
\hline & $6 \times 1014$ & 6 & Lemma 6.11 \\
\hline & $9 \times 676$ & 4 & $\begin{array}{l}\text { Lemma 6.16; } \\
m=3, s=26\end{array}$ \\
\hline & 607 & 3 & Lemma 6.16, \\
\hline & & 3 & $m=2, s=39$ \\
\hline & $13 \times 468$ & 6 & Lemma 6.11 \\
\hline & $18 \times 338$ & & \\
\hline & $26 \times 234$ & 6 & Lemma 6.11 \\
\hline & $36 \times 169$ & & \\
\hline & $39 \times 156$ & 6 & Lemma 6.11 \\
\hline & $52 \times 117$ & 3 & $\begin{array}{c}\text { Lemma } 6.20 \\
t=13,3-(4,9 ; 6)\end{array}$ \\
\hline & $78 \times 78$ & 6 & [18] \\
\hline
\end{tabular}

\begin{tabular}{|c|c|c|c|}
\hline$n$ & $a \times b$ & $N$ & Construction \\
\hline 79 & $79 \times 79$ & $\checkmark$ & Corollary 6.13 \\
\hline \multirow[t]{13}{*}{80} & $2 \times 3200$ & 9 & Lemma 6.11 \\
\hline & $4 \times 1600$ & 9 & Lemma 6.11 \\
\hline & $5 \times 1280$ & 9 & Lemma $\overline{6.11}$ \\
\hline & $8 \times 800$ & 9 & Lemma 6.11 \\
\hline & $10 \times 640$ & 9 & Lemma 6.11 \\
\hline & $16 \times 400$ & 9 & Lemma 6.11 \\
\hline & $20 \times 320$ & 9 & Lemma 6.11 \\
\hline & $25 \times 256$ & 4 & $\begin{array}{l}\text { Lemma } 6.22 \\
h=5, k=16\end{array}$ \\
\hline & $32 \times 200$ & 3 & $\begin{array}{l}\text { Lemma 6.22 } \\
h=4, k=20\end{array}$ \\
\hline & $40 \times 160$ & 9 & Lemma 6.11 \\
\hline & $50 \times 128$ & 4 & $\begin{array}{l}\text { Lemma } 6.22 \\
h=5, k=16\end{array}$ \\
\hline & $64 \times 100$ & 3 & $\begin{array}{l}\text { Lemma } 6.22 \\
h=4, k=20\end{array}$ \\
\hline & $80 \times 80$ & 9 & {$[2,5]$} \\
\hline 81 & All possible & $\checkmark$ & $\begin{array}{lll}\text { Corollary } 6.13 \\
\end{array}$ \\
\hline \multirow[t]{4}{*}{82} & $2 \times 3362$ & 8 & Lemma 6.11 \\
\hline & $4 \times 1681$ & 3 & $\begin{array}{l}\text { Lemma } 6.16 \\
m=2, s=41\end{array}$ \\
\hline & $41 \times 164$ & 8 & Lemma 6.11 \\
\hline & $82 \times 82$ & 8 & [18] \\
\hline 83 & $83 \times 83$ & $\checkmark$ & $\begin{array}{lll}\text { Corollary } & 6.13 \\
\end{array}$ \\
\hline
\end{tabular}




\begin{tabular}{|c|c|c|c|}
\hline$n$ & $a \times b$ & $N$ & Construction \\
\hline \multirow[t]{28}{*}{84} & $2 \times 3528$ & 6 & Lemma 6.11 \\
\hline & $3 \times 2352$ & 6 & Lemma 6.11 \\
\hline & $4 \times 1764$ & 6 & Lemma $\overline{6.11}$ \\
\hline & $6 \times 1176$ & 6 & Lemma 6.11 \\
\hline & $7 \times 1008$ & 6 & Lemma $\longdiv { 6 . 1 1 }$ \\
\hline & $8 \times 882$ & 3 & Lemma 6.22 \\
\hline & $0 \times 002$ & & $h=4, k=21$ \\
\hline & $9 \times 784$ & 4 & Lemma 6.16; \\
\hline & & & $m=3, s=28$ \\
\hline & $12 \times 588$ & 6 & Lemma 6.11 \\
\hline & $14 \times 504$ & 6 & Lemma $\overline{6.11}$ \\
\hline & $16 \times 441$ & 3 & Lemma 6.22 \\
\hline & & & $h=4, k=21$ \\
\hline & $18 \times 392$ & & \\
\hline & $21 \times 336$ & 6 & Lemma 6.11 \\
\hline & $24 \times 294$ & 3 & Lemma 6.22 \\
\hline & & & $h=4, k=21$ \\
\hline & $28 \times 252$ & 6 & Lemma 6.11 \\
\hline & $36 \times 106$ & 3 & Lemma 6.22 \\
\hline & $30 \times 190$ & & $h=4, k=21$ \\
\hline & $42 \times 168$ & 6 & Lemma 6.11 \\
\hline & $49 \times 144$ & 5 & Lemma 6.22 \\
\hline & & & $h=7, k=12$ \\
\hline & $56 \times 126$ & 5 & $\begin{array}{l}\text { Lemma } 6.22 \\
h=7, k=12\end{array}$ \\
\hline & $63 \times 112$ & 5 & $\begin{array}{c}\text { Lemma 6.22 } \\
h=7, k=12\end{array}$ \\
\hline & 08 & 3 & Lemma 6.22, \\
\hline & 10 & 5 & $h=4, k=21$ \\
\hline & $84 \times 84$ & 6 & [18] \\
\hline \multirow[t]{4}{*}{85} & $5 \times 1445$ & 6 & Lemma 6.11 \\
\hline & $17 \times 425$ & 6 & Lemma 6.11 \\
\hline & $25 \times 289$ & 4 & Lemma 6.22; \\
\hline & $85 \times 85$ & 6 & [18] \\
\hline \multirow[t]{4}{*}{86} & $2 \times 3698$ & 6 & Lemma 6.11 \\
\hline & $4 \times 1849$ & 3 & Lemma 6.16 \\
\hline & $43 \times 172$ & 6 & Lemma 6.11 \\
\hline & $86 \times 86$ & 6 & [18] \\
\hline
\end{tabular}

\begin{tabular}{|c|c|c|c|}
\hline$n$ & $a \times b$ & $N$ & Construction \\
\hline \hline 87 & $3 \times 2523$ & 6 & Lemma 6.11 \\
& $9 \times 841$ & & \\
& $29 \times 261$ & 6 & Lemma 6.11 \\
& $87 \times 87$ & 6 & {$[18$} \\
\hline 88 & $2 \times 3872$ & 7 & Lemma 6.11 \\
& $4 \times 1936$ & 7 & Lemma \\
& $8 \times 968$ & 7 & Lemma \\
& $11 \times 704$ & 7 & Lemma \\
& $16 \times 484$ \\
& 16.11 \\
& $22 \times 352$ & 7 & Lemma 6.22 \\
& $32 \times 242$ & 7 & Lemma 6.11 \\
& $44 \times 176$ & 7 & Lemma \\
& 64.22 \\
& $64 \times 121$ & 7 & Lemma 6.11 \\
& $88 \times 88$ & 7 & $h=8, k=11$ \\
\hline 89 & $89 \times 89$ & $\checkmark$ & Corollary \\
\hline
\end{tabular}




\begin{tabular}{|c|c|c|c|}
\hline$n$ & $a \times b$ & $N$ & Construction \\
\hline \multirow[t]{28}{*}{$\overline{90}$} & $2 \times 4050$ & $\overline{6} 6$ & Lemma 6.11 \\
\hline & $3 \times 2700$ & 6 & Lemma 6.11 \\
\hline & $4 \times 2025$ & 3 & Lemma 6.16, \\
\hline & & & $m=2, s=45$ \\
\hline & $5 \times 1620$ & 6 & Lemma 6.11 \\
\hline & $6 \times 1350$ & 6 & Lemma 6.11 \\
\hline & $9 \times 900$ & 6 & Lemma 6.11 \\
\hline & $10 \times 810$ & 6 & Lemma 6.11 \\
\hline & $12 \times 675$ & 3 & Lemma 6.16 \\
\hline & $15 \times 540$ & 6 & Lemma 6.11 \\
\hline & $18 \times 450$ & 6 & Lemma 6.11 \\
\hline & $20 \times 405$ & 3 & Lemma 6.22 \\
\hline & $20 \times 405$ & 3 & $h=5, k=18$ \\
\hline & $25 \times 244$ & 3 & Lemma 6.22, \\
\hline & $2.5 \times 324$ & 5 & $h=5, k=18$ \\
\hline & $27 \times 300$ & & \\
\hline & $30 \times 270$ & 6 & Lemma 6.11 \\
\hline & $36 \times 225$ & 3 & Lemma 6.22, \\
\hline & & & $h=18, k=5$ \\
\hline & $45 \times 180$ & 6 & Lemma 6.11 \\
\hline & $50 \times 162$ & 3 & Lemma 6.22 \\
\hline & & & $h=5, k=18$ \\
\hline & $54 \times 150$ & 3 & Lemma 6.22; \\
\hline & & & $h=18, k=5$ \\
\hline & $60 \times 135$ & 3 & Lemma 6.22; \\
\hline & & & $h=5, k$ \\
\hline & $81 \times 100$ & & \\
\hline & $90 \times 90$ & 6 & [18] \\
\hline \multirow[t]{5}{*}{91} & $7 \times 1183$ & 7 & \begin{tabular}{l|l|} 
Lemma 6.11 \\
\end{tabular} \\
\hline & $13 \times 637$ & 7 & Lemma 6.11 \\
\hline & $49 \times 169$ & 6 & Lemma 6.22 \\
\hline & & & $h=7, k=13$ \\
\hline & $91 \times 91$ & 7 & [18] \\
\hline \multirow[t]{8}{*}{92} & $2 \times 4232$ & 6 & Lemma 6.11 \\
\hline & $4 \times 2116$ & 6 & Lemma 6.11 \\
\hline & $8 \times 1058$ & 3 & Lemma 6.22 \\
\hline & & & $\begin{array}{c}h=4, k=23 \\
\text { Lemma } 6.22\end{array}$ \\
\hline & $16 \times 529$ & 3 & $h=4, k=23$ \\
\hline & $23 \times 368$ & 6 & Lemma 6.11 \\
\hline & $46 \times 184$ & 6 & Lemma \\
\hline & $92 \times 92$ & 6 & [18] \\
\hline
\end{tabular}

\begin{tabular}{|c|c|c|c|}
\hline$n$ & $a \times b$ & $N$ & Construction \\
\hline \multirow[t]{4}{*}{93} & $3 \times 2883$ & 6 & Lemma 6.11 \\
\hline & $9 \times 961$ & & \\
\hline & $31 \times 279$ & 6 & Lemma 6.11 \\
\hline & $93 \times 93$ & 6 & [18] \\
\hline \multirow[t]{5}{*}{94} & $2 \times 4418$ & 6 & Lemma 6.11 \\
\hline & $4 \times 2209$ & 3 & Lemma 6.16; \\
\hline & & & $m=2, s=47$ \\
\hline & $47 \times 188$ & 6 & Lemma 6.11 \\
\hline & $94 \times 94$ & 6 & [18] \\
\hline \multirow[t]{5}{*}{95} & $5 \times 1805$ & 6 & $\begin{array}{ll}\text { Lemma } 6.11 \\
\end{array}$ \\
\hline & $19 \times 475$ & 6 & Lemma 6.11 \\
\hline & $25 \times 361$ & 4 & Lemma 6.22 \\
\hline & & 6 & $h=b, k=19$ \\
\hline & $20 \times 30$ & 0 & {$[10]$} \\
\hline \multirow[t]{16}{*}{96} & $2 \times 4608$ & 7 & Lemma 6.11 \\
\hline & $3 \times 3072$ & 7 & Lemma 6.11 \\
\hline & $4 \times 2304$ & 7 & Lemma 6.11 \\
\hline & $6 \times 1536$ & 7 & Lemma 6.11 \\
\hline & $8 \times 1152$ & 7 & Lemma 6.11 \\
\hline & $9 \times 1024$ & & \\
\hline & $12 \times 768$ & 7 & Lemma 6.11 \\
\hline & $16 \times 576$ & 7 & Lemma 6.11 \\
\hline & $18 \times 512$ & & \\
\hline & $24 \times 384$ & 7 & Lemma 6.11 \\
\hline & $32 \times 288$ & 7 & Lemma 6.11 \\
\hline & $36 \times 256$ & 5 & $\begin{array}{l}\text { Lemma 6.22; } \\
h=4, k=24\end{array}$ \\
\hline & $48 \times 192$ & 7 & Lemma 6.11 \\
\hline & $64 \times 144$ & 5 & $\begin{array}{l}\text { Lemma } 6.22 \\
h=4, k=24\end{array}$ \\
\hline & $72 \times 128$ & 5 & $\begin{array}{l}\text { Lemma 6.22: } \\
h=4, k=24\end{array}$ \\
\hline & $96 \times 96$ & 7 & [18] \\
\hline 97 & $97 \times 97$ & $\checkmark$ & Corollary 6.13 \\
\hline
\end{tabular}




\begin{tabular}{|c|c|c|c|}
\hline$n$ & $a \times b$ & $N$ & Construction \\
\hline \multirow[t]{9}{*}{98} & $2 \times 4802$ & 6 & Lemma 6.11 \\
\hline & $4 \times 2401$ & 3 & Lemma 6.16. \\
\hline & & & $m=2, s=49$ \\
\hline & $7 \times 1372$ & 6 & Lemma 6.11 \\
\hline & $14 \times 686$ & 6 & Lemma 6.11 \\
\hline & $28 \times 343$ & 3 & Lemma \\
\hline & $28 \times 343$ & & $h=7, k=14$ \\
\hline & $49 \times 196$ & 6 & Lemma 6.11 \\
\hline & $98 \times 98$ & 6 & [18] \\
\hline \multirow[t]{9}{*}{99} & $3 \times 3267$ & 8 & Lemma 6.11 \\
\hline & $9 \times 1089$ & 8 & Lemma $\overline{6.11}$ \\
\hline & $11 \times 891$ & 8 & Lemma 6.11 \\
\hline & $27 \times 363$ & 8 & Lemma 6.22 \\
\hline & & & $h=9, k=11$ \\
\hline & $33 \times 297$ & 8 & Lemma 6.11 \\
\hline & $81 \times 121$ & 8 & Lemma 6.22 \\
\hline & $01 \times 121$ & & $h=9, k=11$ \\
\hline & $99 \times 99$ & 8 & [18] \\
\hline \multirow[t]{11}{*}{100} & $2 \times 5000$ & 8 & $\begin{array}{ll}\text { Lemma } 6.11 \\
\end{array}$ \\
\hline & $4 \times 2500$ & 8 & Lemma $\overline{\overline{6.11}}$ \\
\hline & $5 \times 2000$ & 8 & Lemma 6.11 \\
\hline & $8 \times 1250$ & 3 & $\begin{array}{l}\text { Lemma 6.22 } \\
h=4 k=25\end{array}$ \\
\hline & $10 \times 1000$ & 8 & Lemma 6.11 \\
\hline & $16 \times 625$ & 3 & Lemma 6.22 \\
\hline & $20 \times 500$ & 8 & Lemma 6.11 \\
\hline & $25 \times 400$ & 8 & Lemma \\
\hline & $50 \times 200$ & 8 & Lemma 6.11 \\
\hline & $80 \times 125$ & 4 & Lemma 6.22 \\
\hline & $100 \times 100$ & 8 & [18] \\
\hline 101 & $101 \times 101$ & $\checkmark$ & Corollary 6.13 \\
\hline
\end{tabular}

\begin{tabular}{|c|c|c|c|}
\hline$n$ & $a \times b$ & $N$ & Construction \\
\hline \multirow[t]{17}{*}{102} & $2 \times 5202$ & 6 & Lemma 6.11 \\
\hline & $3 \times 3468$ & 6 & Lemma 6.11 \\
\hline & $4 \times 2601$ & 3 & Lemma 6.16; \\
\hline & $4 \times 2001$ & & $m=2, s=51$ \\
\hline & $6 \times 1734$ & 6 & Lemma 6.11 \\
\hline & $9 \times 1156$ & & \\
\hline & $12 \times 867$ & 3 & Lemma 6.16; \\
\hline & 1 & & $3=51$ \\
\hline & $17 \times 612$ & 6 & Lemma 6.11 \\
\hline & $18 \times 578$ & 3 & Lemma 6.17 \\
\hline & $34 \times 306$ & & $=34$ \\
\hline & $34 \times 306$ & 6 & Lemma 6.11 \\
\hline & $36 \times 289$ & & \\
\hline & $51 \times 204$ & 6 & Lemma 6.11 \\
\hline & $68 \times 153$ & 3 & Lemma 6.20 \\
\hline & & & $t=17,3-(4,9 ; 6)$ \\
\hline & $102 \times 102$ & 6 & [18] \\
\hline 103 & $103 \times 103$ & $\checkmark$ & \begin{tabular}{|l|l|} 
Corollary & 6.13 \\
\end{tabular} \\
\hline \multirow[t]{11}{*}{104} & $2 \times 5408$ & 7 & Lemma 6.11 \\
\hline & $4 \times 2704$ & 7 & Lemma $\overline{\overline{6.11}}$ \\
\hline & $8 \times 1352$ & 7 & Lemma $\overline{\underline{6.11}}$ \\
\hline & $13 \times 832$ & 7 & Lemma $\overline{6.11}$ \\
\hline & $16 \times 676$ & 7 & Lemma 6.22 \\
\hline & & & $h=8, k=13$ \\
\hline & $26 \times 416$ & 7 & Lemma 6.11 \\
\hline & $32 \times 338$ & 7 & Lemma 6.22 \\
\hline & & & $h=8, k=13$ \\
\hline & $52 \times 208$ & 7 & Lemma 6.11 \\
\hline & $104 \times 104$ & 7 & [18] \\
\hline
\end{tabular}




\begin{tabular}{|c|c|c|c|}
\hline$n$ & $a \times b$ & $N$ & Construction \\
\hline \multirow[t]{17}{*}{105} & $3 \times 3675$ & 7 & Lemma 6.11 \\
\hline & $5 \times 2205$ & 7 & Lemma 6.11 \\
\hline & $7 \times 1575$ & 7 & Lemma 6.11 \\
\hline & $9 \times 1225$ & 4 & Lemma 6.16 \\
\hline & & & $m=3, s=35$ \\
\hline & $15 \times 735$ & 7 & Lemma 6.11 \\
\hline & $21 \times 525$ & 7 & Lemma $\overline{6.11}$ \\
\hline & $25 \times 441$ & 4 & Lemma 6.22 \\
\hline & & & $h=5, k=21$ \\
\hline & $35 \times 315$ & 7 & Lemma 6.11 \\
\hline & $45 \times 245$ & 4 & Lemma 6.22 \\
\hline & $40 \div 025$ & & Lemma 6.22, \\
\hline & 225 & 4 & $h=7, k=15$ \\
\hline & $63 \times 175$ & 4 & $\begin{array}{l}\text { Lemma 6.22; } \\
h=7, k=15\end{array}$ \\
\hline & 47 & 4 & Lemma 6.22, \\
\hline & & & $h=5, k=21$ \\
\hline & $105 \times 105$ & 7 & [18] \\
\hline \multirow[t]{4}{*}{106} & $2 \times 5618$ & 6 & Lemma 6.11 \\
\hline & $4 \times 2809$ & 3 & Lemma 6.16 \\
\hline & $53 \times 212$ & 6 & $\begin{array}{c}m=2, s=53 \\
\text { Lemma } 6.11\end{array}$ \\
\hline & $106 \times 106$ & 6 & [18] \\
\hline 107 & $107 \times 107$ & $\checkmark$ & Corollary 6.13 \\
\hline
\end{tabular}

\begin{tabular}{|c|c|c|c|}
\hline$n$ & $a \times b$ & $N$ & Construction \\
\hline \multirow[t]{20}{*}{108} & $2 \times 5832$ & 6 & Lemma 6.11 \\
\hline & $3 \times 3888$ & 6 & Lemma 6.11 \\
\hline & $4 \times 2916$ & 6 & Lemma \\
\hline & $6 \times 1944$ & 6 & Lemma 6.11 \\
\hline & $9 \times 1296$ & 6 & Lemma 6.11 \\
\hline & $12 \times 972$ & 6 & Lemma 6.11 \\
\hline & $16 \times 729$ & 3 & Lemma 6.22 \\
\hline & 10 त & & $h=4, k=27$ \\
\hline & $18 \times 648$ & 6 & Lemma 6.11 \\
\hline & $24 \times 486$ & 3 & Lemma 6.22 \\
\hline & & & $h=4, k=27$ \\
\hline & $27 \times 432$ & 6 & Lemma 6.11 \\
\hline & $36 \times 324$ & 6 & Lemma 6.11 \\
\hline & $48 \times 243$ & 3 & Lemma 6.22; \\
\hline & $40 \times 240$ & 5 & $h=4, k=27$ \\
\hline & $54 \times 216$ & 6 & Lemma 6.11 \\
\hline & $72 \times 162$ & 5 & Lemma 6.22 \\
\hline & & & $h=9, k=12$ \\
\hline & $81 \times 144$ & 5 & $h=9 k=12$ \\
\hline & $108 \times 108$ & 6 & [18] \\
\hline 109 & $109 \times 109$ & $\checkmark$ & \begin{tabular}{l|l} 
Corollary & 6.13
\end{tabular} \\
\hline \multirow[t]{16}{*}{110} & $2 \times 6050$ & 6 & Lemma 6.11 \\
\hline & $4 \times 3025$ & 3 & Lemma 6.16 \\
\hline & $4 \times 3025$ & 3 & $m=2, s=55$ \\
\hline & $5 \times 2420$ & 6 & Lemma 6.11 \\
\hline & $10 \times 1210$ & 6 & Lemma 6.11 \\
\hline & $11 \times 1100$ & 6 & Lemma 6.11 \\
\hline & $20 \times 605$ & 3 & Lemma 6.22 \\
\hline & $22 \times 550$ & 6 & $\begin{array}{c}h=5, k=22 \\
\text { Lemma } 6.11\end{array}$ \\
\hline & $22 \times 000$ & & Lemma \\
\hline & $25 \times 484$ & 3 & $h=5, k=22$ \\
\hline & $44 \times 275$ & & \\
\hline & $50 \times 242$ & 3 & Lemma 6.22; \\
\hline & $55 \times 220$ & 6 & Lemma 611 \\
\hline & & & Lemma \\
\hline & $100 \times 121$ & 3 & $h=5, k=22$ \\
\hline & $110 \times 110$ & 6 & [18] \\
\hline
\end{tabular}




\begin{tabular}{|c|c|c|c|}
\hline$n$ & $a \times b$ & $N$ & Construction \\
\hline \multirow[t]{5}{*}{111} & $3 \times 4107$ & 6 & \begin{tabular}{ll|} 
Lemma 6.11 \\
\end{tabular} \\
\hline & $9 \times 1369$ & 4 & Lemma 6.16 , \\
\hline & & & $m=3, s=3$ \\
\hline & $37 \times 333$ & 6 & Lemma $6.11 \mid$ \\
\hline & $111 \times 111$ & 6 & [18] \\
\hline \multirow[t]{15}{*}{112} & $2 \times 6272$ & 13 & Lemma 6.11 \\
\hline & $4 \times 3136$ & 13 & Lemma 6.11 \\
\hline & $7 \times 1792$ & 13 & Lemma $\overline{\text { 6.11 }}$ \\
\hline & $8 \times 1568$ & 13 & Lemma 6.11 \\
\hline & $14 \times 896$ & 13 & Lemma \\
\hline & $16 \times 784$ & 13 & Lemma 6.11 \\
\hline & $28 \times 448$ & 13 & Lemma \\
\hline & $32 \times 392$ & 6 & Lemma 6.22 \\
\hline & 02 人 58 & & $h=16, k=7$ \\
\hline & $49 \times 256$ & 6 & $\begin{array}{l}\text { Lemma 6.22, } \\
h=7 . k=16\end{array}$ \\
\hline & $56 \times 224$ & 13 & Lemma 6.11 \\
\hline & $64 \times 196$ & 6 & Lemma 6.22 \\
\hline & & & $h=16, k=7$ \\
\hline & $98 \times 128$ & 6 & Lemma 6.22 \\
\hline & $112 \times 112$ & 13 & {$[18]$} \\
\hline 113 & $113 \times 113$ & $\checkmark$ & Corollary 6.13 \\
\hline \multirow[t]{14}{*}{114} & $2 \times 6498$ & 6 & Lemma 6.11 \\
\hline & $3 \times 4332$ & 6 & Lemma 6.11 \\
\hline & $4 \times 3249$ & 3 & Lemma 6.16. \\
\hline & & & $m=2, s=57$ \\
\hline & $6 \times 2166$ & 6 & Lemma 6.11 \\
\hline & $9 \times 1444$ & & \\
\hline & $12 \times 1083$ & 3 & $\begin{array}{l}\text { Lemma 6.16 } \\
m=2, s=57\end{array}$ \\
\hline & $18 \times 722$ & 4 & Lemma 6.16, \\
\hline & $19 \times 684$ & 6 & $m=3, s=38$ \\
\hline & $36 \times 361$ & & Lemma|b.11 \\
\hline & $38 \times 342$ & 6 & Lemma 6.11 \\
\hline & $57 \times 228$ & 6 & Lemma 6.11 \\
\hline & $76 \times 171$ & 3 & Lemma 6.20 \\
\hline & $114 \times 114$ & 6 & $t=19,3-(4,9 ; 6)$ \\
\hline & & 0 & \\
\hline
\end{tabular}

\begin{tabular}{|c|c|c|c|}
\hline$n$ & $a \times b$ & $N$ & Construction \\
\hline \multirow[t]{5}{*}{115} & $5 \times 2645$ & 7 & $\begin{array}{ll}\text { Lemma } 6.11 \\
\end{array}$ \\
\hline & $23 \times 575$ & 7 & Lemma $\overline{\overline{6.11}}$ \\
\hline & $25 \times 529$ & 4 & Lemma 6.22 \\
\hline & & & $h=5, k=23$ \\
\hline & $115 \times 115$ & 7 & [18] \\
\hline \multirow[t]{8}{*}{116} & $2 \times 6728$ & 6 & Lemma 6.11 \\
\hline & $4 \times 3364$ & 6 & Lemma 6.11 \\
\hline & $8 \times 168 ?$ & 3 & Lemma 6.22 \\
\hline & $0 \times 1002$ & & $h=4, k=29$ \\
\hline & $16 \times 841$ & 3 & Lemma 6.22: \\
\hline & $29 \times 464$ & 6 & Lemma 6.11 \\
\hline & $58 \times 232$ & 6 & Lemma $\overline{6.11}$ \\
\hline & $116 \times 116$ & 6 & [18] \\
\hline \multirow[t]{9}{*}{117} & $3 \times 4563$ & 8 & Lemma 6.11 \\
\hline & $9 \times 1521$ & 8 & Lemma $\overline{\overline{6.11}}$ \\
\hline & $13 \times 1053$ & 8 & Lemma 6.11 \\
\hline & $27 \times 507$ & 8 & Lemma 6.22 \\
\hline & & & $h=9, k=13$ \\
\hline & $39 \times 351$ & 8 & Lemma 6.11 \\
\hline & $81 \times 160$ & 8 & Lemma 6.22 \\
\hline & & & $h=9, k=13$ \\
\hline & $117 \times 117$ & 8 & [18] \\
\hline \multirow[t]{5}{*}{118} & $2 \times 6962$ & 6 & Lemma 6.11 \\
\hline & $4 \times 3481$ & 3 & Lemma 6.16 \\
\hline & & & $m=2, s=59$ \\
\hline & $59 \times 236$ & 6 & Lemma 6.11 \\
\hline & $118 \times 118$ & 6 & [18] \\
\hline \multirow[t]{4}{*}{119} & $7 \times 2023$ & 6 & Lemma 6.11 \\
\hline & $17 \times 833$ & 6 & Lemma 6.11 \\
\hline & $49 \times 289$ & 6 & Lemma 6.22 \\
\hline & $119 \times 119$ & 6 & [18] \\
\hline
\end{tabular}




\begin{tabular}{|c|c|c|c|}
\hline$n$ & $a \times b$ & $N$ & Construction \\
\hline \multirow[t]{34}{*}{120} & $2 \times 7200$ & 7 & Lemma 6.11 \\
\hline & $3 \times 4800$ & 7 & Lemma 6.11 \\
\hline & $4 \times 3600$ & 7 & Lemma 6.11 \\
\hline & $5 \times 2880$ & 7 & Lemma 6.11 \\
\hline & $6 \times 2400$ & 7 & Lemma 6.11 \\
\hline & $8 \times 1800$ & 7 & Lemma 6.11 \\
\hline & $9 \times 1600$ & & \\
\hline & $10 \times 1440$ & 7 & Lemma 6.11 \\
\hline & $12 \times 1200$ & 7 & Lemma 6.11 \\
\hline & $15 \times 960$ & 7 & Lemma 6.11 \\
\hline & $16 \times 900$ & 4 & Lemma 6.22 \\
\hline & $18 \times 800$ & & $n=8, k=15$ \\
\hline & $20 \times 720$ & 7 & Lemma 6.11 \\
\hline & $24 \times 600$ & 7 & Lemma 6.11 \\
\hline & $25 \times 576$ & 4 & $\begin{array}{l}\text { Lemma } 6.22 \\
h=5, k=24\end{array}$ \\
\hline & $30 \times 480$ & 7 & Lemma 6.11 \\
\hline & $32 \times 450$ & 4 & Lemma 6.22 \\
\hline & & & Lemma 6.22 , \\
\hline & $36 \times 400$ & 4 & $h=4, k=30$ \\
\hline & $40 \times 360$ & 7 & Lemma 6.11 \\
\hline & $45 \times 320$ & 4 & $\begin{array}{l}\text { Lemma 6.22; } \\
h=5, k=24\end{array}$ \\
\hline & $48 \times 300$ & 4 & $\begin{array}{l}\text { Lemma 6.22, } \\
h=8, k=15\end{array}$ \\
\hline & $50 \times 288$ & 4 & $\begin{array}{l}\text { Lemma } 6.22 \\
h=5, k=24\end{array}$ \\
\hline & $60 \times 240$ & 7 & Lemma 6.11 \\
\hline & $64 \times 225$ & 4 & Lemma 6.22 \\
\hline & & & Lemma 6.22 \\
\hline & 200 & 4 & $h=8, k=15$ \\
\hline & $75 \times 192$ & 4 & $\begin{array}{l}\text { Lemma 6.22, } \\
h=5, k=24\end{array}$ \\
\hline & $80 \times 180$ & 4 & $\begin{array}{l}\text { Lemma } 6.22 \\
h=8, k=15\end{array}$ \\
\hline & $90 \times 160$ & 4 & Lemma 6.22, \\
\hline & & & $\begin{array}{l}h=5, k=24 \\
\text {. }\end{array}$ \\
\hline & $96 \times 150$ & 4 & $h=8, k=15$ \\
\hline & $100 \times 144$ & 4 & Lemma 6.22: \\
\hline & $120 \times 120$ & 7 & {$[18]$} \\
\hline
\end{tabular}

\begin{tabular}{|c|c|c|c|}
\hline$n$ & $a \times b$ & $N$ & Construction \\
\hline 121 & $121 \times 121$ & $\checkmark$ & Corollary 6.13 \\
\hline \multirow[t]{4}{*}{122} & $2 \times 7442$ & 6 & Lemma 6.11 \\
\hline & $4 \times 3721$ & 3 & Lemma 6.16; \\
\hline & $61 \times 244$ & 6 & Lemma 6.11 \\
\hline & $122 \times 122$ & 6 & [18] \\
\hline \multirow[t]{4}{*}{123} & $3 \times 5043$ & 6 & Lemma 6.11 \\
\hline & $9 \times 1681$ & & \\
\hline & $41 \times 369$ & 6 & Lemma 6.11 \\
\hline & $123 \times 123$ & 6 & [18] \\
\hline \multirow[t]{7}{*}{124} & $2 \times 4688$ & 6 & Lemma 6.11 \\
\hline & $4 \times 3844$ & 6 & Lemma 6.11 \\
\hline & $8 \times 1922$ & 3 & $\begin{array}{l}\text { Lemma 6.22; } \\
h=4, k=31\end{array}$ \\
\hline & $16 \times 961$ & 3 & $\begin{array}{l}\text { Lemma } 6.22 \\
h=4, k=31\end{array}$ \\
\hline & $31 \times 496$ & 6 & Lemma 6.11 \\
\hline & $62 \times 248$ & 6 & Lemma 6.11 \\
\hline & $124 \times 124$ & 6 & [18] \\
\hline 125 & All possible & $\checkmark$ & \begin{tabular}{l|l} 
Corollary 6.13 \\
\end{tabular} \\
\hline
\end{tabular}




\begin{tabular}{|c|c|c|c|}
\hline$n$ & $a \times b$ & $N$ & Construction \\
\hline \multirow[t]{26}{*}{126} & $2 \times 7638$ & 6 & Lemma 6.11 \\
\hline & $3 \times 5292$ & 6 & Lemma 6.11 \\
\hline & $4 \times 3969$ & 3 & Lemma 6.16 \\
\hline & $4 \times 5909$ & & $m=2, s=63$ \\
\hline & $6 \times 2646$ & 6 & Lemma 6.11 \\
\hline & $7 \times 2268$ & 6 & Lemma 6.11 \\
\hline & $9 \times 1764$ & 6 & Lemma 6.11 \\
\hline & $12 \times 1323$ & 3 & Lemma 6.16 \\
\hline & $14 \times 1134$ & 6 & Lemma 6.11 \\
\hline & $18 \times 882$ & 6 & Lemma 6.11 \\
\hline & $21 \times 756$ & 6 & Lemma 6.11 \\
\hline & $27 \times 588$ & 3 & Lemma 6.22 \\
\hline & & & $\begin{array}{l}n=9, \kappa=14 \\
\text { Lemma } 6.22\end{array}$ \\
\hline & $28 \times 56 r$ & 3 & $h=7, k=18$ \\
\hline & $36 \times 441$ & 3 & Lemma 6.22, \\
\hline & 年 & & $h=9, k=14$ \\
\hline & $42 \times 378$ & 6 & Lemma 6.11 \\
\hline & $49 \times 324$ & 3 & $\begin{array}{l}\text { Lemma } 6.22 \\
h=7, k=18\end{array}$ \\
\hline & $54 \times$ & 3 & Lemma 6.22; \\
\hline & 4 & 3 & $h=9, k=14$ \\
\hline & $63 \times 252$ & 6 & Lemma 6.11 \\
\hline & $81 \times 196$ & 3 & Lemma 6.22 \\
\hline & & & $h=9, k=14$ \\
\hline & $84 \times 189$ & 3 & $\begin{array}{l}\text { Lemma } 0.22 \beta \\
h=7, k=18\end{array}$ \\
\hline & $98 \times 162$ & 3 & Lemma 6.22; \\
\hline & $126 \times 126$ & 6 & {$[18]$} \\
\hline 127 & $127 \times 127$ & $\checkmark$ & Corollary 6.13 \\
\hline 128 & All possible & $\checkmark$ & Corollary 6.13 \\
\hline 129 & $3 \times 5547$ & 7 & Lemma 6.11 \\
\hline & $9 \times 1849$ & & \\
\hline & $43 \times 387$ & 7 & Lemma 6.11 \\
\hline & $129 \times 129$ & 7 & [18] \\
\hline
\end{tabular}

\begin{tabular}{|c|c|c|c|}
\hline$n$ & $a \times b$ & $N$ & Construction \\
\hline \multirow[t]{16}{*}{130} & $2 \times 8450$ & 6 & Lemma 6.11 \\
\hline & $4 \times 4225$ & 3 & Lemma 6.16; \\
\hline & & & $m=2, s=65$ \\
\hline & $5 \times 3380$ & 6 & Lemma 6.11 \\
\hline & $10 \times 1690$ & 6 & Lemma 6.11 \\
\hline & $13 \times 1300$ & 6 & Lemma 6.11 \\
\hline & $20 \times 845$ & 4 & Lemma 6.22 \\
\hline & $25 \times 676$ & 4 & Lemma 6.22; \\
\hline & $20 \times 010$ & 4 & $h=5, k=26$ \\
\hline & $26 \times 650$ & 6 & Lemma 6.11 \\
\hline & $50 \times 338$ & 4 & Lemma 6.22 \\
\hline & & & Lemma 6.20 \\
\hline & $52 \times 325$ & 3 & $\begin{array}{c}t=13 \\
3-(4,25 ; 10)\end{array}$ \\
\hline & $65 \times 260$ & 6 & Lemma 6.11 \\
\hline & $100 \times 169$ & 4 & Lemma 6.22 \\
\hline & $130 \times 130$ & 6 & [18] \\
\hline 131 & $131 \times 131$ & $\checkmark$ & Corollary 6.13 \\
\hline
\end{tabular}




\begin{tabular}{|c|c|c|c|}
\hline$n$ & $a \times b$ & $N$ & Construction \\
\hline \multirow[t]{28}{*}{132} & $2 \times 8712$ & $\overline{76}$ & Lemma $\overline{6.11}$ \\
\hline & $3 \times 5808$ & 6 & Lemma 6.11 \\
\hline & $4 \times 4356$ & 6 & Lemma \\
\hline & $6 \times 2904$ & 6 & Lemma $\overline{6.11}$ \\
\hline & & 3 & Lemma 6.22 \\
\hline & $8 \times 2178$ & 3 & $h=4, k=33$ \\
\hline & $9 \times 1936$ & 4 & Lemma 6.16 \\
\hline & $9 \times 1930$ & 4 & $m=3, s=44$ \\
\hline & $11 \times 1584$ & 6 & Lemma 6.11 \\
\hline & $12 \times 1452$ & 6 & Lemma \\
\hline & $16 \times 1089$ & 3 & Lemma 6.22 \\
\hline & $10 \times 1009$ & & $h=4, k=33$ \\
\hline & $18 \times 968$ & & \\
\hline & $22 \times 792$ & 6 & Lemma 6.11 \\
\hline & $24 \times 726$ & 5 & Lemma 6.22 \\
\hline & $24 \times 126$ & 5 & $h=12, k=11$ \\
\hline & $33 \times 528$ & 6 & Lemma 6.11 \\
\hline & $36 \times 484$ & 5 & Lemma 6.22 \\
\hline & & & $h=12, k=11$ \\
\hline & $44 \times 396$ & 6 & Lemma 6.11 \\
\hline & $48 \times 363$ & 5 & Lemma 6.22 \\
\hline & & & $h=12, k=11$ \\
\hline & $66 \times 264$ & 6 & Lemma 6.11 \\
\hline & $88 \times 198$ & 5 & Lemma 6.22 \\
\hline & & & $h=11, k=12$ \\
\hline & $99 \times 176$ & 5 & $\begin{array}{c}\text { Lemma } 6.22 \\
h=11, k=12\end{array}$ \\
\hline & $121 \times 144$ & 5 & Lemma 6.22: \\
\hline & & 6 & $n=11, k=12$ \\
\hline \multirow[t]{5}{*}{133} & $7 \times 2527$ & 7 & Lemma 6.11 \\
\hline & $19 \times 937$ & 7 & Lemma \\
\hline & $10 \times 361$ & 6 & Lemma \\
\hline & & & $h=7, k=19$ \\
\hline & $133 \times 133$ & 7 & [18] \\
\hline \multirow[t]{5}{*}{134} & $2 \times 8978$ & 6 & Lemma 6.11 \\
\hline & $4 \times 4489$ & 3 & Lemma 6.16 \\
\hline & & & $m=2, s=67$ \\
\hline & $67 \times 268$ & 6 & Lemma 6.11 \\
\hline & $134 \times 134$ & 6 & [18] \\
\hline
\end{tabular}

\begin{tabular}{|c|c|c|c|}
\hline$n$ & $a \times b$ & $N$ & Construction \\
\hline \multirow[t]{11}{*}{135} & $3 \times 6075$ & 7 & Lemma 6.11 \\
\hline & $5 \times 3645$ & 7 & Lemma 6.11 \\
\hline & $9 \times 2025$ & 7 & Lemma 6.11 \\
\hline & $15 \times 1215$ & 7 & Lemma 6.11 \\
\hline & $25 \times 729$ & 4 & Lemma 6.22 \\
\hline & $27 \times 675$ & 7 & Lemma 6.11 \\
\hline & $45 \times 405$ & 7 & Lemma 6.11 \\
\hline & $75 \times 243$ & 4 & Lemma 6.22; \\
\hline & & & $h=5, k=27$ \\
\hline & $81 \times 225$ & 4 & $\begin{array}{l}\text { Lemma } \\
h=27, k=5\end{array}$ \\
\hline & $135 \times 135$ & 7 & [18] \\
\hline \multirow[t]{10}{*}{136} & $2 \times 9248$ & 7 & Lemma 6.11 \\
\hline & $4 \times 4624$ & 7 & Lemma 6.11 \\
\hline & $8 \times 2312$ & 7 & Lemma 6.11 \\
\hline & $16 \times 1156$ & 7 & Lemma 6.22 \\
\hline & $17 \times 1088$ & 7 & Lemma 6.11 \\
\hline & $32 \times 578$ & 7 & Lemma 6.22 \\
\hline & $34 \times 544$ & 7 & Lemma 6.11 \\
\hline & $64 \times 289$ & 7 & Lemma 6.22 \\
\hline & $68 \times 272$ & 7 & $\begin{array}{l}h=8, k=17 \\
\text { Lemma } 6.11\end{array}$ \\
\hline & $136 \times 136$ & 7 & [18] \\
\hline 137 & $137 \times 137$ & $\checkmark$ & Corollary 6.13 \\
\hline
\end{tabular}




\begin{tabular}{|c|c|c|c|}
\hline$n$ & $a \times b$ & $N$ & Construction \\
\hline \multirow[t]{15}{*}{138} & $2 \times 9522$ & 6 & Lemma 6.11 \\
\hline & $3 \times 6348$ & 6 & Lemma 6.11 \\
\hline & $4 \times 4761$ & 3 & Lemma 6.16; \\
\hline & $4 \times 4 / 01$ & & $m=2, s=69$ \\
\hline & $6 \times 3174$ & 6 & Lemma 6.11 \\
\hline & $9 \times 2116$ & 4 & Lemma 6.16; \\
\hline & & & Lemma 6.16 \\
\hline & $12 \times 1587$ & 3 & $m=2, s=69$ \\
\hline & $18 \times 1058$ & & \\
\hline & $23 \times 828$ & 6 & Lemma 6.11 \\
\hline & $36 \times 529$ & & \\
\hline & $46 \times 414$ & 6 & Lemma 6.11 \\
\hline & $69 \times 276$ & 6 & Lemma 6.11 \\
\hline & $92 \times 207$ & 3 & $\begin{array}{c}\text { Lemma 6.20; } \\
t=23,3-(4,9 ; 6)\end{array}$ \\
\hline & $138 \times 138$ & 6 & [18] \\
\hline 139 & $139 \times 139$ & $\checkmark$ & Corollary 6.13 \\
\hline
\end{tabular}

\begin{tabular}{|c|c|c|c|}
\hline$n$ & $a \times b$ & $N$ & Construction \\
\hline \multirow[t]{30}{*}{140} & $2 \times 9800$ & 6 & Lemma 6.11 \\
\hline & $4 \times 4900$ & 6 & Lemma 6.11 \\
\hline & $5 \times 3920$ & 6 & Lemma 6.11 \\
\hline & $7 \times 2800$ & 6 & Lemma 6.11 \\
\hline & $8 \times 2450$ & 3 & Lemma 6.22 \\
\hline & & & $h=4, k=35$ \\
\hline & $10 \times 1960$ & 6 & Lemma 6.11 \\
\hline & $14 \times 1400$ & 6 & Lemma 6.11 \\
\hline & $16 \times 1225$ & 3 & Lemma 6.22 \\
\hline & & & $h=4, k=35$ \\
\hline & $20 \times 980$ & 6 & Lemma 6.11 \\
\hline & $25 \times 784$ & 4 & $\begin{array}{l}\text { Lemma } 0.22 \\
h=5, k=28\end{array}$ \\
\hline & $28 \times 700$ & 6 & Lemma 6.11 \\
\hline & $35 \times 560$ & 6 & Lemma 6.11 \\
\hline & $40 \times 490$ & 4 & Lemma 6.22; \\
\hline & $40 \times 490$ & 4 & $h=5, k=28$ \\
\hline & $49 \times 400$ & 4 & Lemma 6.22 \\
\hline & & & Lemma 6.22, \\
\hline & $50 \times 392$ & 4 & $h=5, k=28$ \\
\hline & $56 \times 350$ & 4 & Lemma 6.22, \\
\hline & טנת ג & & $h=7, k=20$ \\
\hline & $70 \times 280$ & 6 & Lemma 6.11 \\
\hline & $80 \times 245$ & 4 & Lemma 6.22; \\
\hline & & & $h=5, k=28$ \\
\hline & $98 \times 200$ & 4 & Lemma 6.22; \\
\hline & & & $\begin{array}{l}n=r, \kappa=20 \\
\text { Lemma } 622\end{array}$ \\
\hline & $100 \times 196$ & 4 & $h=5, k=28$ \\
\hline & $112 \times 175$ & 4 & Lemma 6.22; \\
\hline & & & $h=7, k=20$ \\
\hline & $140 \times 140$ & 6 & [18] \\
\hline \multirow[t]{4}{*}{141} & $3 \times 6627$ & 7 & Lemma 6.11 \\
\hline & $9 \times 2209$ & & \\
\hline & $47 \times 423$ & 7 & Lemma 6.11 \\
\hline & $141 \times 141$ & 7 & [18] \\
\hline \multirow[t]{4}{*}{142} & $2 \times 10082$ & 6 & Lemma 6.11 \\
\hline & $4 \times 5041$ & 3 & Lemma 6.16 \\
\hline & $71 \times 284$ & 6 & Lemma 6.11 \\
\hline & $142 \times 142$ & 6 & [18] \\
\hline
\end{tabular}




\begin{tabular}{|c|c|c|c|}
\hline$n$ & $a \times b$ & $N$ & Construction \\
\hline \multirow[t]{5}{*}{143} & $11 \times 1859$ & 10 & Lemma 6.11 \\
\hline & $13 \times 1573$ & 10 & Lemma \\
\hline & $121 \times 169$ & 10 & Lemma 6.22 \\
\hline & & & $h=11, k=13$ \\
\hline & $143 \times 143$ & 10 & [18] \\
\hline \multirow[t]{24}{*}{144} & $2 \times 10368$ & 10 & Lemma 6.11 \\
\hline & $3 \times 6912$ & 10 & Lemma \\
\hline & $4 \times 5184$ & 10 & Lemma \\
\hline & $6 \times 3456$ & 10 & Lemma \\
\hline & $8 \times 2592$ & 10 & Lemma \\
\hline & $9 \times 2304$ & 10 & Lemma 6.11 \\
\hline & $12 \times 1728$ & 10 & Lemma \\
\hline & $16 \times 1296$ & 10 & Lemma 6.11 \\
\hline & $18 \times 1152$ & 10 & Lemma \\
\hline & $24 \times 864$ & 10 & Lemma 6.11 \\
\hline & $27 \times 768$ & 8 & Lemma 6.22 \\
\hline & $32 \times 648$ & 8 & $\begin{array}{l}h=9, k=16 \\
\text { Lemma } 6.22 \\
h=16, k=9\end{array}$ \\
\hline & $36 \times 576$ & 10 & Lemma 6.11 \\
\hline & $48 \times 432$ & 10 & Lemma 6.11 \\
\hline & $54 \times 384$ & 8 & Lemma 6.22 \\
\hline & & & $\begin{array}{l}n=9, k=16 \\
\text { Lemma } 6.22\end{array}$ \\
\hline & $64 \times 324$ & 8 & $h=16, k=9$ \\
\hline & $72 \times 288$ & 10 & Lemma 6.11 \\
\hline & $81 \times 256$ & 8 & $\begin{array}{l}\text { Lemma 6.22 } \\
h=9 k=16\end{array}$ \\
\hline & $96 \times 216$ & 8 & $\begin{array}{l}\text { Lemma } 6.22 \\
h=16, k=9\end{array}$ \\
\hline & $108 \times 192$ & 8 & $\begin{array}{l}\text { Lemma 6.22 } \\
h=9, k=16\end{array}$ \\
\hline & $128 \times 162$ & 8 & Lemma 6.22 , \\
\hline & & & $h=16, k=9$ \\
\hline & $144 \times 144$ & 10 & \\
\hline 150 & $36 \times 625$ & & \\
\hline 174 & $36 \times 841$ & & \\
\hline 186 & $36 \times 961$ & & \\
\hline 210 & $36 \times 1225$ & & \\
\hline
\end{tabular}

$$
C=\text { Computational construction }
$$




\section{Bibliography}

[1] R. Abel, $V(12, t)$ vectors and other designs from difference and quasi-difference matrices, preprint (2006).

[2] __ On the existence of balanced incomplete block designs and transversal designs, Ph.D. thesis, University of New South Wales, 1995.

[3] R. Abel and F. Bennett, The existence of 2-SOLSSOOMs, in Designs 2002, W. D. Wallis, ed., Kluwer, Boston, 2003.

[4] R. Abel and N. Cavenagh, Concerning eight mutually orthogonal Latin squares, J. Combin. Des. 15 (2006), no. 3, 255-261.

[5] R. Abel and Y. Cheng, Some new MOLS of order $2^{n} p$ for $p$ a prime power, Australas. J. Combin. 10 (1994), 175-186.

[6] R. Abel, C. Colbourn, and M. Wojtas, Concerning seven and eight mutually orthogoanl Latin squares, J. Combin. Des. 12 (2004), 123-131.

[7] R. Abel and G. Ge, Some difference matrix constructions and an almost completion for the existence of triplewhist tournaments twh(v), Europ. J. Combin. 26 (2005), 1094-1104.

[8] R. Abel and D. Todorov, Four MOLS of order 20,30,38 and 44, J. Combin. Thoery A 64 (1994), 144-148.

[9] R. Abel, H. Zhang, and X. Zhang, Three mutually orthogonal idempotent Latin squares of orders 22 and 26, J. Stat. Plann. Infer. 51 (1996), 101-106.

[10] I. Anderson, Combinatorial Designs: Construction Methods, Ellis Horwood, England, 1990.

[11] A. Assaf, I. Bluskov, M. Greig, and N. Shalaby, Block designs with block size 5 with higher index, preprint.

[12] R. Bos and S. Shrikhande, On the construction of sets of mutually orthogonal latin squares using a computer, Technometrics 2 (1960), 507-516.

[13] R. Bose, S. Shrikhande, and E. Parker, Rurther results on the construction of mutually orthogonal latin squares and falsity of Euler's conjecture, Canad. J. Math. 12 (1960), 189-203.

[14] H. Cao, J. Dinitz, D. Kreher, D. Stinson, and R. Wei, On orthogonal generalized equitable rectangles, Des. Codes Cryptogr. 51 (2009), no. 3, 225-230. 
[15] S. Chowla, P. Erdös, and E. Straus, On the maximal number of pairwise orthogonal latin squares of a given order, Canadian J. Math. 12 (1960), 207-208.

[16] W. Cochran and G. Cox, Experimental designs, John Wiley \& Sons Inc., New York, 1957, 2nd ed.

[17] C. Colbourn, Four MOLS of order 26, J. Combin. Math. Combin. Comput. 17 (1995), 147148.

[18] C. Colbourn and J. Dinitz, Making the MOLS table, in Computational and Constructive Design Theory, W. D. Wallis, ed., Kluwer, Dordrecht, 1996.

[19] _ The CRC handbook of combinatorial designs, Discrete Mathematics, CRC Press, Boca Raton, 2006.

[20] R. Diestel, Graph theory, Graduate Texts in Mathematics, vol. 173, Springer, New York, 2000.

[21] L. Euler, Recherches sur une nouvelle espéce de quarrés magiques, Verh. Zeeuw. Gen. Weten. Vlissengen 9 (1782), 85-239.

[22] S. Furino and T. Lee, A translation of J. X. Lu's existance theory for resolvable block designs, J. Combin. Designs 3 (1995), 321-340.

[23] S. Furino, Y. Miao, and J. Yin, Frames and resolvable designs, CRC Press Series on Discrete Mathematics and its Applications, CRC Press, Boca Raton, FL, 1996.

[24] G. Ge and A. C. Ling, Asymptotic results on the existence of 4-RGDDs and uniform 5-GDDs, J. Combin. Des. 13 (2005), 222-237.

[25] G. Ge, R. Rees, and L. Zhu, Group divisible designs with block size four and group-type $g^{u} m^{1}$ with $m$ as large or as small as possible, Journal of Combinatorial Theory Series A 98 (2002), 357 Ü376.

[26] R. Gupta, Studies in graph theory., Chap. 2. The chromatic index and the degree of a graph, Notices Amer. Math. Soc. 13 (1966), 714-719.

[27] P. Hall, On representatives of subsets, J. London Math. Soc. 10 (1935), 26-30.

[28] H. Hanani, Balanced incomplete block designs and related designs, Discrete Math. 11 (1975), 255-369.

[29] S. Hurd and D. Sarvate, An edge-coloring problem for designs, Bulletin of the ICA 49 (2007), $15-20$.

[30] D. Johnson, A. Dulmage, and N. Mendelsohn, Orthomorphisms of groups of orthogonal Latin squares, Canad. J. Math. 13 (1961), 356-172.

[31] T. Kirkman, On a problem in combinations, Cambridge and Dublin Math. J. 2 (1847), 191204.

[32] _ _ Query VI, Lady's and Gentleman's Diary 48 (1850). 
[33] H. MacNeish, Euler squares, Ann. of Math. 23 (1922), 221-227.

[34] H. Mann, The construction of orthogonal latin squares, Ann. Math. Statist. 13 (1942), 418423.

[35] D. Marx, Graph coloring problems, John von Neumann PhD students Conference, Budapest University of Technology and Economics, 2003.

[36] Y. Miao and L. Zhu, On resolvable BIBDs with block size five, Ars Combin. 39 (1995), 261275.

[37] W. Mills, Some mutually orthogonal Latin squares, Congr. Numer. 19 (1977), 473-487.

[38] E. Moore, Tactical memoranda i-iii, Amer. J. Math. 18 (1896), 264-303.

[39] S. Nakano, T. Nishizeki, and X. Zhou, Edge-coloring algorithms, in Computer Science Today: Recent Trends and Developments, (Jan van Leeuwen ed.), LNCS 1000 (1995), 172-183.

[40] A. Nazarok, Five pairwise orthogonal Latin squares of order 21, Issled. oper. i (1991), 54-56.

[41] E. Parker, Constructions of some sets of mutually orthogonal latin squares, Proc. Amer. Math. Soc. 10 (1959), 946-949.

[42] __ Nonextendability conditions on mutually orthogonal latin squares, Proc. Amer. Math. Soc. 13 (1962), 219-221.

[43] T. Ristenpart and P. Rogaway, How to enrich the message space of a cipher, Lect. Notes Comput. Sc. 4593 (2007), 101-118.

[44] P. Schellenberg, G. Van Rees, and S. Vanstone, Four pairwise orthogonal Latin squares of order 15, Ars Combin. 6 (1978), 141-150.

[45] D. Stinson, Generalized mix functions and orthogonal equitable rectangles, Des. Codes Cryptogr. 45 (2007), no. 3, 347-357.

[46] D. Todorov, Three mutually orthogonal Latin squares of order 14, Ars Combin. 20 (1985), 45-48.

[47] __ Four mutually orthogonal Latin squares of order 20, Ars Combin. 27 (1989), 63-65.

[48] V. Vizing, On an estimate of the chromatic class of a p-graph, Diskret. Analiz No. 3 (1964), $25-30$.

[49] S. Wang, On self-orthogonal Latin squares and partial transversals of Latin squares, Ph.D. thesis, Ohio State University, 1978.

[50] D. West, Introduction to graph theory, Prentice Hall Inc., Upper Saddle River, NJ, 1996.

[51] R. Wilson, An existence theory for pairwise balanced designs I. Composition theorems and morphisms, Journal of Combinatorial Theory A 13 (1972), 220-245.

[52] _ Concerning the number of mutually orthogonal latin squares, Discrete Math. 9 (1974), 181-198. 
[53] M. Wojtas, Five mutually orthogonal Latin squares of order 35, J. Combin. Des. 4 (1996), $153-154$.

[54] _ Three new constructions of mutually orthogonal Latin squares, J. Combin. Des. 8 (2000), 218-220.

[55] L. Zhu, Some recent developments on BIBDs and related designs, Discrete Math. 123 (1993), 189-214. 


\section{Index}

$K_{v}, 1$

$N(S), 79$

$N_{\text {MOELR }}, 88$

$N_{\text {MOLR }}, 88$

$N_{\text {MOLS }}(n), 86$

$P_{k}, 82$

$\mathcal{B}, 4,12$

$\mathrm{BIBD}, 4$

$\Delta(G), 81$

$\mathrm{LS}(n), 85$

MOELR, 88

$\operatorname{MOELR}(a, b ; n), 88$

MOLR, 88

OGER, 89

PBD, 86

RBIBD, 9

TS, 5

$\mathcal{V}, 4$

$\left(\begin{array}{l}\mathcal{V} \\ 2\end{array}\right), 18$

$\chi^{\prime}(G), 81$

$\chi(G), 2$

$\lambda, 4$

$k-\mathrm{PD}, 82$

adjacent, 2

alternating path, 4

augmenting path, 4

balanced incomplete block design, 4 blocks, 4

complete, 4

parameters, 5

bipartite graph, 3

$X, Y$-bigraph, 79

chromatic number, 2

circulant matrix, 17

$g$-circulant, 64

coloring $k$-edge-colorable, 81

$k$-edge-coloring, 81

$k$-coloring, 2

equitably colored, 82

proper $k$-edge-coloring, 81

proper coloring, 2

semi-properly colored BIBD, 25

column-regular, 88

complete graph, 1

equitable rectangle, 82

finite field construction, 91

graph, 1

conflict graph, 2

simple graph, 1

group divisible design, 6

blocks, 6

groups, 6

transverse pair, 7

uniform, 7

incidence matrix, 15

edge-coloring incidence matrix, 17

edge-incidence matrix, 16

incident, 81

Latin rectangle, 88

equitable, 88

Latin square, 21, 85

$n$-set, 85

order, 85

side, 85

loop, 1

marriage condition, 79

matching, 2

maximal matching, 79

perfect matching, 80 
monochromatic, 11

multiple edges, 1

mutually orthogonal, 88

mutually orthogonal equitable Latin rectangles, 88

mutually orthogonal Latin squares, 86

NP-complete, 4

orthogonal, 86,88

pairwise balanced design, 86

panchromatic, 2

parallel class, 9

path decomposition, 82

permutation, 89

regular, 88

repetition number, 16

resolution class, 9

resolvable balanced incomplete block design, 9

row-regular, 88

saturation, 79

Steiner triple system, 5

transversal design, 87

triple system, 5 